\title{
Optimal Entropy-Transport problems and a new Hellinger-Kantorovich distance between positive measures
}

\author{
Matthias Liero ${ }^{1}$ - Alexander Mielke ${ }^{1,2}$. \\ Giuseppe Savaré ${ }^{3}$
}

Received: 11 January 2016 / Accepted: 3 September 2017 /

Published online: 14 December 2017

(C) The Author(s) 2017. This article is an open access publication

\begin{abstract}
We develop a full theory for the new class of Optimal EntropyTransport problems between nonnegative and finite Radon measures in general topological spaces. These problems arise quite naturally by relaxing the marginal constraints typical of Optimal Transport problems: given a pair of finite measures (with possibly different total mass), one looks for minimizers of the sum of a linear transport functional and two convex entropy functionals, which quantify in some way the deviation of the marginals of the transport plan from the assigned measures. As a powerful application of this theory,
\end{abstract}

M. Liero: Partially supported by the Einstein Stiftung Berlin via the ECMath/MATHEON project SE2.

A. Mielke: Partially supported by DFG via project C05 within CRC 1114 (Scaling cascades in complex systems) and by ERC via AdG 267802 AnaMultiScale.

G. Savaré: Partially supported by MIUR via PRIN project Calculus of Variations, by Cariplo foundation and Regione Lombardia via project Variational evolution problems and optimal transport, and by IMATI-CNR.

$\bowtie$ Giuseppe Savaré

giuseppe.savare@unipv.it

Matthias Liero

liero@wias-berlin.de

Alexander Mielke

mielke@wias-berlin.de

1 Weierstraß-Institut Für Angewandte Analysis Und Stochastik, Berlin, Germany

2 Humboldt-Universität $\mathrm{Zu}$, Berlin, Germany

3 Dipartimento Di Matematica “F. Casorati”, Università Di Pavia, Pavia, Italy 
we study the particular case of Logarithmic Entropy-Transport problems and introduce the new Hellinger-Kantorovich distance between measures in metric spaces. The striking connection between these two seemingly far topics allows for a deep analysis of the geometric properties of the new geodesic distance, which lies somehow between the well-known Hellinger-Kakutani and Kantorovich-Wasserstein distances.

\section{Contents}

1 Introduction . . . . . . . . . . . . . . . . . . . . . 973

Part I. Optimal Entropy-Transport problems ․ . . . . . . . . . . . . . . 985

2 Preliminaries . . . . . . . . . . . . . . . . . . . . 985

2.1 Measure theoretic notation _ . . . . . . . . . . . . . . . . . 985

2.2 Min-max and duality . . . . . . . . . . . . . . . . . . 987

2.3 Entropy functions and their conjugates $\ldots \ldots \ldots$. . . . . . . . . . 988

2.4 Relative entropy integral functionals _ . . . . . . . . . . . . . . 992

3 Optimal Entropy-Transport problems … . . . . . . . . . . . . . . . . . 1000

3.1 The basic setting . . . . . . . . . . . . . . . . . . . . . 1000

3.2 The primal formulation of the optimal entropy-transport problem . . . . . . . 1001

3.3 Examples . . . . . . . . . . . . . . . . . . . . . . . 1003

3.4 Existence of solutions to the primal problem . . . . . . . . . . . . . 1006

3.5 The reverse formulation of the primal problem . . . . . . . . . . . . . 1010

4 The dual problem . . . . . . . . . . . . . . . . . . . . 1011

4.1 The "inf-sup" derivation of the dual problem in the basic coercive setting . . . . . 1011

4.2 Dual problem and optimality conditions _ . . . . . . . . . . . . . . 1013

4.3 A general duality result . . . . . . . . . . . . . . . . . . . . . 1018

4.4 Existence of optimal Entropy-Kantorovich potentials . . . . . . . . . . . 1024

5 "Homogeneous" formulations of optimal Entropy-Transport problems . . . . . . . . . 1030

5.1 The homogeneous marginal perspective functional . . . . . . . . . . . . 1030

5.2 Entropy-transport problems with "homogeneous" marginal constraints . . . . . 1037

Part II. The Logarithmic Entropy-Transport problem and the Hellinger-Kantorovich distance . . . . . . . . . . . . . . . . . . . . . . . . . . 1041

6 The Logarithmic Entropy-Transport (LET) problem … . . . . . . . . . . . . 1041

6.1 The metric setting for Logarithmic Entropy-Transport problems . . . . . . . . . 1042

6.2 The Logarithmic Entropy-Transport problem: main results . . . . . . . . . . 1043

7 The metric side of the LET-functional: the Hellinger-Kantorovich distance . . . . . . 1050

7.1 The cone construction . . . . . . . . . . . . . . . . . . . . . 1051

7.2 Radon measures in the cone $\mathfrak{C}$ and homogeneous marginals . . . . . . . . 1053

7.3 The Hellinger-Kantorovich problem . . . . . . . . . . . . . . . . . 1056

7.4 Gluing lemma and triangle inequality . . . . . . . . . . . . . . . 1060

7.5 Metric and topological properties . . . . . . . . . . . . . . . . 1064

7.6 Hellinger-Kantorovich distance and Entropy-Transport functionals . . . . . . . 1068

7.7 Limiting cases: recovering the Hellinger-Kakutani distance and the Kantorovich-

Wasserstein distance . . . . . . . . . . . . . . . . . . . . . . . 1075

7.8 The Gaussian Hellinger-Kantorovich distance . . . . . . . . . . . . . . . 1077

8 Dynamic interpretation of the Hellinger-Kantorovich distance . . . . . . . . . . 1078

8.1 Absolutely continuous curves and geodesics in the cone $\mathfrak{C} \ldots \ldots \ldots$. . . . . 1079

8.2 Lifting of absolutely continuous curves and geodesics . . . . . . . . . . 1082

8.3 Lower curvature bound in the sense of Alexandrov . . . . . . . . . . . . . 1086

8.4 Duality and Hamilton-Jacobi equation . . . . . . . . . . . . . . . . . 1089 
8.5 The dynamic interpretation of the Hellinger-Kantorovich distance "à la BenamouBrenier" . . . . . . . . . . . . . . . . . . . . . . 1098

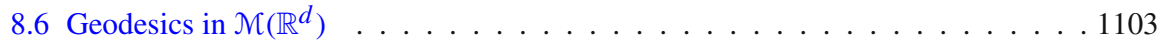

8.7 Contraction properties: convolution and Heat equation in $\operatorname{RCD}(0, \infty)$ metricmeasure spaces

A On the chronological development of our theory f . . . . . . . . . . . . 1113

References . . . . . . . . . . . . . . . . . . . . 1115

\section{Main notation}

$\mathcal{M}(X)$

$\eta_{0}$

$\mathcal{P}(X), \mathcal{P}_{2}(X)$

$\mathcal{B}(X)$

$T_{\sharp} \mu$

$\gamma=\sigma \mu+\mu^{\perp}, \mu=\varrho \gamma+\gamma^{\perp}$

$\mu\llcorner A$

$\mathrm{C}_{b}(X)$

$\operatorname{Lip}_{b}(X), \operatorname{Lip}_{b s}(X)$

$\operatorname{LSC}_{b}(X), \operatorname{LSC}_{s}(X)$

$\mathrm{USC}_{b}(X), \mathrm{USC}_{s}(X)$

$\mathrm{B}(X), \mathrm{B}_{b}(X)$

$\mathrm{L}^{p}(X, \mu), \mathrm{L}^{p}\left(X, \mu ; \mathbb{R}^{d}\right)$

$\Gamma\left(\mathbb{R}_{+}\right)$

$F(s), F_{i}(s)$

$F^{*}(\phi), F_{i}^{*}(\phi)$

$F^{\circ}(\varphi), F_{i}^{\circ}\left(\varphi_{i}\right)$

$R(r), R_{i}\left(r_{i}\right)$

$\mathrm{D}(F)$
Finite positive Radon measures on a Hausdorff topological space $X$

The null measure in some $\mathcal{M}(X)$

Radon probability measures on $X$ (with finite quadratic moment)

Borel subsets of $X$

Push forward of $\mu \in \mathcal{N}(X)$ by a map $T: X \rightarrow Y$, see (2.5)

Lebesgue decompositions of $\gamma$ and $\mu$,

Lemma 2.3

Restriction of a measure to $A: \mu\llcorner A(B)$ $:=\mu(A \cap B)$, Sect. 2.1

Continuous and bounded real functions on $X$

Bounded (with bounded support)

Lipschitz real functions on $X$

Lower semicontinuous and bounded (or simple) real functions on $X$

Upper semicontinuous and bounded (or simple) real functions on $X$

Borel (resp. bounded Borel) real functions

Borel $\mu$-integrable real (or $\mathbb{R}^{d}$-valued) functions

Set of admissible entropy functions, see (2.13), (2.14)

Admissible entropy functions

Legendre transform of $F, F_{i}$, see (2.16)

Concave conjugate of an entropy function, see (2.45)

Reversed entropies, see (2.28)

Proper domain $\{F<\infty\}$ of an extended real function $F$, see (2.14) 


$$
\begin{aligned}
& \stackrel{\mathrm{D}}{ }(F) \\
& F_{\infty}^{\prime}, F_{0}^{\prime}, \operatorname{aff} F_{\infty} \\
& \chi_{K} \\
& H_{c}\left(r_{1}, r_{2}\right), H\left(x_{1}, r_{1} ; x_{2}, r_{2}\right) \\
& \mathrm{C}\left(x_{1}, x_{2}\right) \\
& \mathrm{d}\left(x_{1}, x_{2}\right), \mathrm{d}_{a}\left(x_{1}, x_{2}\right) \\
& \mathscr{F}(\gamma \mid \mu), \mathscr{R}(\mu \mid \gamma) \\
& \mathscr{E}\left(\gamma \mid \mu_{1}, \mu_{2}\right), \mathrm{ET}\left(\mu_{1}, \mu_{2}\right) \\
& \mathscr{D}\left(\varphi \mid \mu_{1}, \mu_{2}\right), \mathrm{D}\left(\mu_{1}, \mu_{2}\right) \\
& \boldsymbol{\Phi}, \Psi
\end{aligned}
$$$$
\text { LET }\left(\mu_{1}, \mu_{2}\right), \ell(\mathrm{d})
$$$$
\mathrm{W}_{\mathrm{d}}\left(\mu_{1}, \mu_{2}\right)
$$$$
\mathrm{H}\left(\mu_{1}, \mu_{2}\right)
$$$$
\mathbf{G K}\left(\mu_{1}, \mu_{2}\right)
$$$$
\left(\mathfrak{C}, \mathrm{d}_{\mathfrak{C}}\right), \mathfrak{o}
$$$$
\mathfrak{C}[r]
$$$$
\mathfrak{h}_{i}^{2}, \operatorname{dil}_{\theta, 2}(\cdot)
$$$$
\mathfrak{H}_{=}^{2}\left(\mu_{1}, \mu_{2}\right), \mathfrak{H}_{\leq}^{2}\left(\mu_{1}, \mu_{2}\right)
$$$$
\mathrm{AC}^{p}([0,1] ; X)
$$$$
\left|\mathrm{x}^{\prime}\right|_{\mathrm{d}}
$$$$
\left|\mathrm{D}_{Z} f\right|,\left|\mathrm{D}_{Z} f\right|_{a}
$$

The interior of $\mathrm{D}(F)$

Recession, derivative at 0 , and asymptotic coefficient of $F$, see (2.15)

The indicator function of a set $K(0$ on $K$ and $+\infty$ outside)

The characteristic function of a set $A$ ( 1 on $A$ and 0 outside)

Marginal perspective function, see (5.1), (5.10), (5.3)

Lower semicontinuous cost function defined in $\boldsymbol{X}=X_{1} \times X_{2}$

A metric on a space $X$ and its truncated version $\mathrm{d} \wedge a$, see (6.8)

Entropy functionals and their reverse form, see (2.35) and (2.57)

General Entropy-Transport functional and its minimum, see (3.4)

Dual functional and its supremum, see (4.10) and (4.8)

Set of admissible Entropy-Kantorovich potentials

Logarithmic Entropy Transport functional and its cost, see Sect. 6.1

Kantorovich-Wasserstein distance in $\mathcal{P}_{2}(X)$

Hellinger-Kantorovich distance in $\mathcal{M}(X)$, see Section 7.3

Gaussian Hellinger-Kantorovich distance in $\mathcal{M}(X)$, see Section 7.8

Metric cone and its vertex, see Section 7.1

Ball of radius $r$ centered at $\mathfrak{o}$ in $\mathfrak{C}$

Homogeneous marginals and dilations, see (7.16), (7.17)

Plans in $\mathfrak{C} \times \mathfrak{C}$ with constrained homogeneous marginals, see (7.21)

Space of curves $\mathrm{x}:[0,1] \rightarrow X$ with $p$-integrable metric speed

Metric speed of a curve $\mathrm{x} \in \mathrm{AC}([a, b]$; $(X, \mathrm{~d}))$, see Sect. 8.1

Metric slope and asymptotic Lipschitz constant in $Z$, see (8.37) 
When no ambiguity is possible, we will often adopt the convention to write the integral of a composition of functions as

$$
\int F(\phi) \mathrm{d} \mu=\int(F \circ \phi) \mathrm{d} \mu=\int F(\phi(x)) \mathrm{d} \mu(x) .
$$

\section{Introduction}

The aim of the present paper is twofold: In Part I we develop a full theory of the new class of Optimal Entropy-Transport problems between nonnegative and finite Radon measures in general topological spaces. As a powerful application of this theory, in Part II we study the particular case of Logarithmic Entropy-Transport problems and introduce the new Hellinger-Kantorovich (HK) distance between measures in metric spaces. The striking connection between these two seemingly far topics is our main focus, and it paves the way for a beautiful and deep analysis of the geometric properties of the geodesic $\mathrm{H}$ distance, which (as our proposed name suggests) can be understood as an inf-convolution of the well-known Hellinger-Kakutani and the KantorovichWasserstein distances, see Remark 8.19 for a discussion of inf-convolutions of distances. In fact, our approach to the theory was opposite: in trying to characterize $\mathrm{H}$, we were first led to the Logarithmic Entropy-Transport problem, see Section A.

From Transport to Entropy-Transport problems. In the classical Kantorovich formulation, Optimal Transport problems [2,40,49,50] deal with minimization of a linear cost functional

$$
\mathscr{C}(\boldsymbol{\gamma})=\int_{X_{1} \times X_{2}} \mathrm{c}\left(x_{1}, x_{2}\right) \mathrm{d} \boldsymbol{\gamma}\left(x_{1}, x_{2}\right), \quad \mathrm{c}: X_{1} \times X_{2} \rightarrow \mathbb{R},
$$

among all the transport plans, i.e. probability measures in $\mathcal{P}\left(X_{1} \times X_{2}\right), \gamma$ whose marginals $\mu_{i}=\pi_{\sharp}^{i} \gamma \in \mathcal{P}\left(X_{i}\right)$ are prescribed. Typically, $X_{1}, X_{2}$ are Polish spaces, $\mu_{i}$ are given Borel measures (but the case of Radon measures in Hausdorff topological spaces has also been considered, see $[26,40])$, the cost function $\mathrm{C}$ is a lower semicontinuous (or even Borel) function, possibly assuming the value $+\infty$, and $\pi^{i}\left(x_{1}, x_{2}\right)=x_{i}$ are the projections on the $i$-th coordinate, so that

$$
\pi_{\sharp}^{i} \boldsymbol{\gamma}=\mu_{i} \Leftrightarrow\left\{\begin{array}{l}
\mu_{1}\left(A_{1}\right)=\boldsymbol{\gamma}\left(A_{1} \times X_{2}\right), \\
\mu_{2}\left(A_{2}\right)=\boldsymbol{\gamma}\left(X_{1} \times A_{2}\right)
\end{array} \text { for every } A_{i} \in \mathcal{B}\left(X_{i}\right) .\right.
$$

Starting from the pioneering work of Kantorovich, an impressive theory has been developed in the last two decades: from one side, typical intrin- 
sic questions of linear programming problems concerning duality, optimality, uniqueness and structural properties of optimal transport plans have been addressed and fully analyzed. In a parallel way, this rich general theory has been applied to many challenging problems in a variety of fields (probability and statistics, functional analysis, PDEs, Riemannian geometry, nonsmooth analysis in metric spaces, just to mention a few of them: since it is impossible here to give an even partial account of the main contributions, we refer to the books $[42,50]$ for a more detailed overview and a complete list of references).

The class of Entropy-Transport problems, we are going to study, arises quite naturally if one tries to relax the marginal constraints $\pi_{\sharp}^{i} \gamma=\mu_{i}$ by introducing suitable penalizing functionals $\mathscr{F}_{i}$, that quantify in some way the deviation from $\mu_{i}$ of the marginals $\gamma_{i}:=\pi_{\sharp}^{i} \boldsymbol{\gamma}$ of $\boldsymbol{\gamma}$. In this paper we consider the general case of integral functionals (also called Csiszàr $f$-divergences [17]) of the form

$$
\begin{aligned}
& \mathscr{F}_{i}\left(\gamma_{i} \mid \mu_{i}\right):=\int_{X_{i}} F_{i}\left(\sigma_{i}\left(x_{i}\right)\right) \mathrm{d} \mu_{i}+\left(F_{i}\right)_{\infty}^{\prime} \gamma_{i}^{\perp}\left(X_{i}\right), \\
& \sigma_{i}=\frac{\mathrm{d} \gamma_{i}}{\mathrm{~d} \mu_{i}}, \quad \gamma_{i}=\sigma_{i} \mu_{i}+\gamma_{i}^{\perp},
\end{aligned}
$$

where $F_{i}:[0, \infty) \rightarrow[0, \infty]$ are given convex entropy functions and $\left(F_{i}\right)_{\infty}^{\prime}$ are their recession constants, see (2.15). Typical examples are the logarithmic or power-like entropies

$$
\begin{aligned}
& U_{p}(s):=\frac{1}{p(p-1)}\left(s^{p}-p(s-1)-1\right), \quad p \in \mathbb{R} \backslash\{0,1\}, \\
& U_{0}(s):=s-1-\log s, \quad U_{1}(s):=s \log s-s+1,
\end{aligned}
$$

or for the total variation functional corresponding to the nonsmooth entropy $V(s):=|s-1|$, considered in [38]. We shall see that the presence of the singular part $\gamma_{i}^{\perp}$ in the Lebesgue decomposition of $\gamma_{i}$ in (1.3) does not force $F_{i}(s)$ to be superlinear as $s \uparrow \infty$ and allows for all the exponents $p$ in (1.4).

Once a specific choice of entropies $F_{i}$ and of finite nonnegative Radon measures $\mu_{i} \in \mathcal{M}\left(X_{i}\right)$ is given, the Entropy-Transport problem can be formulated as

$$
\mathrm{ET}\left(\mu_{1}, \mu_{2}\right):=\inf \left\{\mathscr{E}\left(\boldsymbol{\gamma} \mid \mu_{1}, \mu_{2}\right): \boldsymbol{\gamma} \in \mathcal{M}\left(X_{1} \times X_{2}\right)\right\},
$$

where $\mathscr{E}$ is the convex functional

$$
\mathscr{E}\left(\boldsymbol{\gamma} \mid \mu_{1}, \mu_{2}\right):=\mathscr{F}_{1}\left(\gamma_{1} \mid \mu_{1}\right)+\mathscr{F}_{2}\left(\gamma_{2} \mid \mu_{2}\right)+\int_{X_{1} \times X_{2}} \mathrm{c}\left(x_{1}, x_{2}\right) \mathrm{d} \boldsymbol{\gamma} .
$$


Notice that the entropic formulation allows for measures $\mu_{1}, \mu_{2}$ and $\boldsymbol{\gamma}$ with possibly different total mass.

The flexibility in the choice of the entropy functions $F_{i}$ (which may also take the value $+\infty$ ) covers a wide spectrum of situations (see Sect. 3.3 for various examples) and in particular guarantees that (1.5) is a real generalization of the classical optimal transport problem, which can be recovered as a particular case of (1.6) when $F_{i}(s)$ is the indicator function of $\{1\}$ (i.e. $F_{i}(s)$ always takes the value $+\infty$ with the only exception of $s=1$, where it vanishes).

Since we think that the structure (1.6) of Entropy-Transport problems will lead to new and interesting models and applications, we have tried to establish their basic theory in the greatest generality, by pursuing the same line of development of Transport problems: in particular we will obtain general results concerning existence, duality and optimality conditions.

Considering e.g. the Logarithmic Entropy case, where $F_{i}(s)=s \log s-$ $s+1$, the dual formulation of (1.5) is given by

$$
\begin{aligned}
& \mathrm{D}\left(\mu_{1}, \mu_{2}\right):=\sup \left\{\mathscr{D}\left(\varphi_{1}, \varphi_{2} \mid \mu_{1}, \mu_{2}\right): \varphi_{i}: X_{i} \rightarrow \mathbb{R},\right. \\
& \left.\varphi_{1}\left(x_{1}\right)+\varphi_{2}\left(x_{2}\right) \leq \mathrm{c}\left(x_{1}, x_{2}\right)\right\}, \\
& \text { where } \mathscr{D}\left(\varphi_{1}, \varphi_{2} \mid \mu_{1}, \mu_{2}\right):=\int_{X_{1}}\left(1-\mathrm{e}^{-\varphi_{1}}\right) \mathrm{d} \mu_{1}+\int_{X_{2}}\left(1-\mathrm{e}^{-\varphi_{2}}\right) \mathrm{d} \mu_{2} \text {, }
\end{aligned}
$$

where one can immediately recognize the same convex constraint of Transport problems: the pair of dual potentials $\varphi_{i}$ should satisfy $\varphi_{1} \oplus \varphi_{2} \leq \mathrm{C}$ on $X_{1} \times X_{2}$. The main difference is due to the concavity of the objective functional

$$
\left(\varphi_{1}, \varphi_{2}\right) \mapsto \int_{X_{1}}\left(1-\mathrm{e}^{-\varphi_{1}}\right) \mathrm{d} \mu_{1}+\int_{X_{2}}\left(1-\mathrm{e}^{-\varphi_{2}}\right) \mathrm{d} \mu_{2},
$$

whose form can be explicitly calculated in terms of the Lagrangian conjugates $F_{i}^{*}$ of the entropy functions. Thus (1.7) consists in the supremum of a concave functional on a convex set described by a system of affine inequalities.

The change of variables $\psi_{i}:=1-\mathrm{e}^{-\varphi_{i}}$ transforms (1.7) in the equivalent problem of maximizing the linear functional

$$
\left(\psi_{1}, \psi_{2}\right) \mapsto \sum_{i} \int_{X_{1}} \psi_{1} \mathrm{~d} \mu_{1}+\int_{X_{2}} \psi_{2} \mathrm{~d} \mu_{2}
$$

on the more complicated convex set 


$$
\left\{\left(\psi_{1}, \psi_{2}\right): \psi_{i}: X_{i} \rightarrow(-\infty, 1), \quad\left(1-\psi_{1}\left(x_{1}\right)\right)\left(1-\psi_{2}\left(x_{2}\right)\right) \geq \mathrm{e}^{-\mathrm{c}\left(x_{1}, x_{2}\right)}\right\} .
$$

It will be useful to have both the representations at our disposal: (1.7) naturally appears from the application of the von Neumann min-max principle from a saddle point formulation of the primal problem (1.5). Moreover, (1.8)-(1.10) will play an important role in the dynamic version of a particular case of ET, the Hellinger-Kantorovich distance that we will introduce later on.

We will calculate the dual problem for every choice of $F_{i}$ and show that its value always coincide with $\mathrm{ET}\left(\mu_{1}, \mu_{2}\right)$. The dual problem also provides optimality conditions, that involve the pair of potentials $\left(\varphi_{1}, \varphi_{2}\right)$, the support of the optimal plan $\gamma$ and the densities $\sigma_{i}$ of its marginals $\gamma_{i}$ w.r.t. $\mu_{i}$. For the Logarithmic Entropy Transport problem above, they read

$$
\begin{aligned}
\sigma_{i}>0, \varphi_{i}=-\log \sigma_{i} \quad \mu_{i} \text { a.e. in } X_{i}, \\
\varphi_{1} \oplus \varphi_{2} \leq \mathrm{c} \quad \text { in } X_{1} \times X_{2}, \quad \varphi_{1} \oplus \varphi_{2}=\mathrm{c} \quad \boldsymbol{\gamma} \text {-a.e. in } X_{1} \times X_{2},
\end{aligned}
$$

and they are necessary and sufficient for optimality.

The study of optimality conditions reveals a different behavior between pure transport problems and entropic ones. In particular, the C-cyclical monotonicity of the optimal plan $\boldsymbol{\gamma}$ (which is still satisfied in the entropic case) does not play a crucial role in the construction of the potentials $\varphi_{i}$. When $F_{i}(0)$ are finite (as in the logarithmic case) it is possible to obtain a general existence result of (generalized) optimal potentials even when $c$ takes the value $+\infty$.

A crucial feature of Entropy-Transport problems (which is not shared by the pure transport ones) concerns a third homogeneous formulation, which exhibits new and unexpected properties, in particular concerning the metric and dynamical aspects of such problems. It is related to the 1-homogeneous Marginal Perspective function

$$
H\left(x_{1}, r_{1} ; x_{2}, r_{2}\right):=\inf _{\theta>0}\left(r_{1} F_{1}\left(\theta / r_{1}\right)+r_{2} F_{2}\left(\theta / r_{2}\right)+\theta \mathrm{c}\left(x_{1}, x_{2}\right)\right)
$$

and to the corresponding integral functional

$$
\begin{aligned}
\mathscr{H}\left(\mu_{1}, \mu_{2} \mid \boldsymbol{\gamma}\right):= & \int_{X_{1} \times X_{2}} H\left(x_{1}, \varrho_{1}\left(x_{1}\right) ; x_{2}, \varrho_{2}\left(x_{2}\right)\right) \mathrm{d} \boldsymbol{\gamma} \\
& +\sum_{i} F_{i}(0) \mu_{i}^{\perp}\left(X_{i}\right), \varrho_{i}:=\frac{\mathrm{d} \mu_{i}}{\mathrm{~d} \gamma_{i}}
\end{aligned}
$$


where $\mu_{i}=\varrho_{i} \gamma_{i}+\mu_{i}^{\perp}$ is the "reverse" Lebesgue decomposition of $\mu_{i}$ w.r.t. the marginals $\gamma_{i}$ of $\gamma$. We will prove that

$$
\mathrm{ET}\left(\mu_{1}, \mu_{2}\right)=\min \left\{\mathscr{H}\left(\mu_{1}, \mu_{2} \mid \boldsymbol{\gamma}\right): \boldsymbol{\gamma} \in \mathcal{M}\left(X_{1} \times X_{2}\right)\right\}
$$

with a precise relation between optimal plans. In the Logarithmic Entropy case $F_{i}(s)=s \log s-(s-1)$ the marginal perspective function $H$ takes the particular form

$$
H\left(x_{1}, r_{1} ; x_{2}, r_{2}\right)=r_{1}+r_{2}-2 \sqrt{r_{1} r_{2}} \mathrm{e}^{-\mathrm{c}\left(x_{1}, x_{2}\right) / 2},
$$

which will be the starting point for understanding the deep connection with the Hellinger-Kantorovich distance. Notice that in the case when $X_{1}=X_{2}$ and $\mathrm{C}$ is the singular cost

$$
\mathrm{c}\left(x_{1}, x_{2}\right):= \begin{cases}0 & \text { if } x_{1}=x_{2} \\ +\infty & \text { otherwise }\end{cases}
$$

(1.13) provides an equivalent formulation of the Hellinger-Kakutani distance [22,25], see also Example E.5 in Sect. 3.3.

Other choices, still in the simple class (1.4), give raise to "transport" versions of well known functionals (see e.g. [31] for a systematic presentation): starting from the reversed entropies $F_{i}(s)=s-1-\log s$ one gets

$$
H\left(x_{1}, r_{1} ; x_{2}, r_{2}\right)=r_{1} \log r_{1}+r_{2} \log r_{2}-\left(r_{1}+r_{2}\right) \log \left(\frac{r_{1}+r_{2}}{2+\mathrm{c}\left(x_{1}, x_{2}\right)}\right),
$$

which in the extreme case (1.15) reduces to the Jensen-Shannon divergence [32], a squared distance between measures derived from the celebrated Kullback-Leibler divergence [28]. The quadratic entropy $F_{i}(s)=\frac{1}{2}(s-1)^{2}$ produces

$$
H\left(x_{1}, r_{1} ; x_{2}, r_{2}\right)=\frac{1}{2\left(r_{1}+r_{2}\right)}\left(\left(r_{1}-r_{2}\right)^{2}+h\left(\mathrm{c}\left(x_{1}, x_{2}\right)\right) r_{1} r_{2}\right)
$$

where $h(c)=c(4-c)$ if $0 \leq c \leq 2$ and 4 if $c \geq 2$ : Equation (1.17) can be seen as the transport variant of the triangular discrimination (also called symmetric $\chi^{2}$-measure), based on the Pearson $\chi^{2}$-divergence [31], and still obtained by (1.12) when $\mathrm{c}$ has the form (1.15).

Also nonsmooth cases, as for $V(s)=|s-1|$ associated to the total variation distance (or nonsymmetric choices of $F_{i}$ ) can be covered by the general theory. In the case of $F_{i}(s)=V(s)$ the marginal perspective function is 


$$
\begin{aligned}
H\left(x_{1}, r_{1} ; x_{2}, r_{2}\right) & =r_{1}+r_{2}-\left(2-\mathrm{c}\left(x_{1}, x_{2}\right)\right)_{+}\left(r_{1} \wedge r_{2}\right) \\
& =\left|r_{2}-r_{1}\right|+\left(\mathrm{c}\left(x_{1}, x_{2}\right) \wedge 2\right)\left(r_{1} \wedge r_{2}\right)
\end{aligned}
$$

when $X_{1}=X_{2}=\mathbb{R}^{d}$ with $\mathrm{c}\left(x_{1}, x_{2}\right):=\left|x_{1}-x_{2}\right|$ we recover the generalized Wasserstein distance $W_{1}^{1,1}$ introduced and studied by [38]; it provides an equivalent variational characterization of the flat metric [39].

However, because of our original motivation (see Section A), Part II will focus on the case of the logarithmic entropy $F_{i}=U_{1}$, where $H$ is given by (1.14). We will exploit its relevant geometric applications, reserving the other examples for future investigations.

\section{From the Kantorovich-Wasserstein distance to the Hellinger-Kantorovich}

distance. From the analytic-geometric point of view, one of the most interesting cases of transport problems occurs when $X_{1}=X_{2}=X$ coincide and the cost functional $\mathscr{C}$ is induced by a distance $\mathrm{d}$ on $X$ : in the quadratic case, the minimum value of (1.1) for given measures $\mu_{1}, \mu_{2}$ in the space $\mathcal{P}_{2}(X)$ of probability measures with finite quadratic moment defines the so called $L^{2}$-Kantorovich-Wasserstein distance

$W_{d}^{2}\left(\mu_{1}, \mu_{2}\right):=\inf \left\{\int d^{2}\left(x_{1}, x_{2}\right) d \gamma\left(x_{1}, x_{2}\right): \boldsymbol{\gamma} \in \mathcal{P}(X \times X), \pi_{\sharp}^{i} \boldsymbol{\gamma}=\mu_{i}\right\}$,

which metrizes the weak convergence (with quadratic moments) of probability measures. The metric space $\left(\mathcal{P}_{2}(X), W_{d}\right)$ inherits many geometric features from the underlying $(X, \mathrm{~d})$ (as separability, completeness, length and geodesic properties, positive curvature in the Alexandrov sense, see [2]). Its dynamic characterization in terms of the continuity equation [7] and its dual formulation in terms of the Hopf-Lax formula and the corresponding (sub-)solutions of the Hamilton-Jacobi equation [37] lie at the core of the applications to gradient flows and partial differential equations of diffusion type [2]. Finally, the behavior of entropy functionals as in (1.3) along geodesics in $\left(\mathcal{P}_{2}(X), \mathrm{W}_{\mathrm{d}}\right)$ $[16,35,37]$ encodes a valuable geometric information, with relevant applications to Riemannian geometry and to the recent theory of metric-measure spaces with Ricci curvature bounded from below [3-5,21,34,47,48].

It has been a challenging question to find a corresponding distance (enjoying analogous deep geometric properties) between finite positive Borel measures with arbitrary mass in $\mathcal{N}(X)$. In the present paper we will show that by choosing the particular cost function

$\mathrm{c}\left(x_{1}, x_{2}\right):=\ell\left(\mathrm{d}\left(x_{1}, x_{2}\right)\right), \quad$ where $\ell(\mathrm{d}):= \begin{cases}-\log \left(\cos ^{2}(\mathrm{~d})\right) & \text { if } \mathrm{d}<\pi / 2, \\ +\infty & \text { otherwise, }\end{cases}$ 
the corresponding Logarithmic-Entropy Transport problem

$$
\begin{aligned}
\operatorname{LT}\left(\mu_{1}, \mu_{2}\right):= & \min _{\boldsymbol{\gamma} \in \mathcal{M}(\boldsymbol{X})} \sum_{i} \int_{X}\left(\sigma_{i} \log \sigma_{i}-\sigma_{i}+1\right) \mathrm{d} \mu_{i} \\
& +\int_{X^{2}} \ell\left(\mathrm{d}\left(x_{1}, x_{2}\right)\right) \mathrm{d} \boldsymbol{\gamma}, \quad \sigma_{i}=\frac{\mathrm{d} \gamma_{i}}{\mathrm{~d} \mu_{i}},
\end{aligned}
$$

coincides with a (squared) distance in $\mathcal{M}(X)$ (which we will call HellingerKantorovich distance and denote by $\mathrm{HK}$ ) that can play the same fundamental role like the Kantorovich-Wasserstein distance for $\mathcal{P}_{2}(X)$.

Here is a (still non exhaustive) list of our main results of part II concerning the Hellinger-Kantorovich distance.

(i) The representation (1.13) based on the Marginal Perspective function (1.14) yields

$$
\begin{aligned}
& \text { LET }\left(\mu_{1}, \mu_{2}\right) \\
& \quad=\min \left\{\int\left(\varrho_{1}+\varrho_{2}-2 \sqrt{\varrho_{1} \varrho_{2}} \cos \left(\mathrm{d}\left(x_{1}, x_{2}\right) \wedge \pi / 2\right)\right) \mathrm{d} \gamma: \varrho_{i}=\frac{\mathrm{d} \mu_{i}}{\mathrm{~d} \gamma_{i}}\right\} .
\end{aligned}
$$

By performing the rescaling $r_{i} \mapsto r_{i}^{2}$ we realize that the function $H\left(x_{1}, r_{1}^{2} ; x_{2}, r_{2}^{2}\right)$ is strictly related to the squared (semi)-distance

$$
\mathrm{d}_{\mathfrak{C}^{(}}^{2}\left(x_{1}, r_{1} ; x_{2}, r_{2}\right):=r_{1}^{2}+r_{2}^{2}-2 r_{1} r_{2} \cos \left(\mathrm{d}\left(x_{1}, x_{2}\right) \wedge \pi\right), \quad\left(x_{i}, r_{i}\right) \in X \times \mathbb{R}_{+}
$$

which is the so-called cone distance in the metric cone $\mathfrak{C}$ over $X$, cf. [10]. The latter is the quotient space of $X \times \mathbb{R}_{+}$obtained by collapsing all the points $(x, 0), x \in X$, in a single point $\mathfrak{o}$, called the vertex of the cone. We introduce the notion of "2-homogeneous marginal"

$$
\begin{aligned}
\mu & =\mathfrak{h}^{2} \alpha:=\pi_{\sharp}^{x}\left(r^{2} \alpha\right), \\
\int_{X} \zeta(x) \mathrm{d} \mu & =\int_{\mathfrak{C}} \zeta(x) r^{2} \mathrm{~d} \alpha(x, r) \quad \text { with } \zeta \in \mathrm{C}_{b}(X),
\end{aligned}
$$

to "project" measures $\alpha \in \mathcal{M}(\mathfrak{C})$ on measures $\mu \in \mathcal{M}(X)$. Conversely, there are many ways to "lift" a measure $\mu \in \mathcal{M}(X)$ to $\alpha \in \mathcal{M}(\mathfrak{C})$ (e.g. by taking $\left.\alpha:=\mu \otimes \delta_{1}\right)$. The Hellinger-Kantorovich distance $\mathrm{HK}\left(\mu_{1}, \mu_{2}\right)$ can then be defined by taking the best Kantorovich-Wasserstein distance between all the possible lifts of $\mu_{1}, \mu_{2}$ in $\mathcal{P}_{2}(\mathfrak{C})$, i.e.

$$
\mathrm{H}\left(\mu_{1}, \mu_{2}\right)=\min \left\{\mathrm{W}_{\mathbf{d}_{\mathfrak{C}}}\left(\alpha_{1}, \alpha_{2}\right): \alpha_{i} \in \mathcal{P}_{2}(\mathfrak{C}), \mathfrak{h}^{2} \alpha_{i}=\mu_{i}\right\}
$$


It turns out that (the square of) (1.24) yields an equivalent variational representation of the LET functional. In particular, (1.24) shows that in the case of concentrated measures

$$
\operatorname{LT}\left(a_{1} \delta_{x_{1}}, a_{2} \delta_{x_{2}}\right)=\mathrm{HK}^{2}\left(a_{1} \delta_{x_{1}}, a_{2} \delta_{x_{2}}\right)=\mathrm{d}_{\mathfrak{C}}^{2}\left(x_{1}, a_{1} ; x_{2}, a_{2}\right) .
$$

Notice that (1.24) resembles the very definition (1.18) of the KantorovichWasserstein distance, where now the role of the marginals $\pi_{\sharp}^{i}$ is replaced by the homogeneous marginals $\mathfrak{h}^{2}$. It is a nontrivial part of the equivalence statement to check that the difference between the cut-off thresholds $(\pi / 2$ in (1.21) and $\pi$ in (1.22) does not affect the identity LET $=\mathrm{HK}^{2}$.

(ii) By refining the representation formula (1.24) by a suitable rescaling and gluing technique, we can prove that $(\mathcal{M}(X), \mathrm{HK})$ is a metric space, a property that is not obvious from the LET-representation and depends on a subtle interplay of the entropy functions $F_{i}(\sigma)=\sigma \log \sigma-\sigma+1$ and the cost function $C$ from (1.19). We show that the metric induces the weak convergence of measures in duality with bounded and continuous functions, thus it is topologically equivalent to the flat or Bounded Lipschitz distance [19, Sect. 11.3], see also [27, Thm. 3]. It also inherits the separability, completeness, length and geodesic properties from the correspondent ones of the underlying space $(X, \mathrm{~d})$. On top of that, we will prove a precise superposition principle (in the same spirit of the Kantorovich-Wasserstein one [2, Sect.8], [33]) for general absolutely continuous curves in $(\mathcal{M}(X), \mathrm{HK})$ in terms of dynamic plans in $\mathfrak{C}$ : as a byproduct, we can give a precise characterization of absolutely continuous curves and geodesics as homogeneous marginals of corresponding curves in $\left(\mathcal{P}_{2}(\mathfrak{C}), W_{\mathfrak{d}_{\mathfrak{C}}}\right)$. An interesting consequence of these results concerns the lower curvature bound of $(\mathcal{M}(X), \mathrm{HK})$ in the sense of Alexandrov: it is a positively curved space if and only if $(X, \mathrm{~d})$ is a geodesic space with curvature $\geq 1$.

(iii) The dual formulation of the LET problem provides a dual characterization of $\mathrm{H}$, viz.

$$
\begin{aligned}
& \frac{1}{2} \mathrm{H}^{2}\left(\mu_{1}, \mu_{2}\right)=\sup \left\{\int \mathscr{P}_{1} \xi \mathrm{d} \mu_{2}-\int \xi \mathrm{d} \mu_{1}:\right. \\
& \left.\qquad \xi \in \operatorname{Lip}_{b}(X), \inf _{X} \xi>-1 / 2\right\},
\end{aligned}
$$

where $\left(\mathscr{P}_{t}\right)_{0 \leq t \leq 1}$ is given by the inf-convolution

$$
\mathscr{P}_{t} \xi(x):=\inf _{x^{\prime} \in X} \frac{\xi\left(x^{\prime}\right)}{1+2 t \xi\left(x^{\prime}\right)}+\frac{\sin ^{2}\left(\mathrm{~d}_{\pi / 2}\left(x, x^{\prime}\right)\right)}{2+4 t \xi\left(x^{\prime}\right)}
$$




$$
=\inf _{x^{\prime} \in X} \frac{1}{t}\left(1-\frac{\cos ^{2}\left(\mathrm{~d}_{\pi / 2}\left(x, x^{\prime}\right)\right)}{1+2 t \xi\left(x^{\prime}\right)}\right) .
$$

(iv) By exploiting the Hopf-Lax representation formula for the HamiltonJacobi equation in $\mathfrak{C}$, we will show that for arbitrary initial data $\xi \in$ $\operatorname{Lip}_{b}(X)$ with inf $\xi>-1 / 2$ the function $\xi_{t}:=\mathscr{P}_{t} \xi$ is a subsolution (a solution, if $(X, \mathrm{~d})$ is a length space) of

$$
\partial_{t}^{+} \xi_{t}(x)+\frac{1}{2}\left|\mathrm{D}_{X} \xi_{t}\right|^{2}(x)+2 \xi_{t}^{2}(x) \leq 0 \quad \text { pointwise in } X \times(0,1) \text {. }
$$

If $(X, \mathrm{~d})$ is a length space we thus obtain the characterization

$$
\begin{array}{r}
\frac{1}{2} \mathrm{HK}^{2}\left(\mu_{0}, \mu_{1}\right)=\sup \left\{\int_{X} \xi_{1} \mathrm{~d} \mu_{1}-\int_{X} \xi_{0} \mathrm{~d} \mu_{0}: \xi \in \mathrm{C}^{k}\left([0,1] ; \operatorname{Lip}_{b}(X)\right),\right. \\
\left.\partial_{t} \xi_{t}(x)+\frac{1}{2}\left|\mathrm{D}_{X} \xi_{t}\right|^{2}(x)+2 \xi_{t}^{2}(x) \leq 0 \text { in } X \times(0,1)\right\},
\end{array}
$$

which reproduces, at the level of $H$, the nice link between $W_{d}$ and Hamilton-Jacobi equations. One of the direct applications of (1.27) is a sharp contraction property w.r.t. $\mathrm{HK}$ for the Heat flow in $\operatorname{RCD}(0, \infty)$ metric measure spaces (and therefore in every Riemannian manifold with nonnegative Ricci curvature).

(v) Formula (1.27) clarifies that the $\mathrm{H}$ distance can be interpreted as a sort of inf-convolution (see the Remark 8.19) between the Hellinger (in duality with solutions to the ODE $\partial_{t} \xi+2 \xi_{t}^{2}=0$ ) and the Kantorovich-Wasserstein distance (in duality with (sub-)solutions to $\left.\partial_{t} \xi_{t}(x)+\frac{1}{2}\left|\mathrm{D}_{X} \xi_{t}\right|^{2}(x) \leq 0\right)$. The Hellinger distance

$$
\operatorname{He}^{2}\left(\mu_{1}, \mu_{2}\right)=\int_{X}\left(\sqrt{\varrho_{1}}-\sqrt{\varrho_{2}}\right)^{2} \mathrm{~d} \gamma, \quad \mu_{i}=\varrho_{i} \gamma
$$

corresponds to the $\mathrm{HK}$ functional generated by the discrete distance $\left(\mathrm{d}\left(x_{1}, x_{2}\right)=\pi / 2\right.$ if $\left.x_{1} \neq x_{2}\right)$. We will prove that

$$
\begin{aligned}
& \mathrm{H}\left(\mu_{1}, \mu_{2}\right) \leq \mathrm{He}\left(\mu_{1}, \mu_{2}\right), \quad \mathrm{H} \mathrm{K}\left(\mu_{1}, \mu_{2}\right) \leq \mathrm{W}_{\mathrm{d}}\left(\mu_{1}, \mu_{2}\right), \\
& \mathrm{H}_{n \mathrm{~d}}\left(\mu_{1}, \mu_{2}\right) \uparrow \mathrm{He}\left(\mu_{1}, \mu_{2}\right), \quad n \mathrm{~K}_{\mathrm{d} / n} \uparrow \mathrm{W}_{\mathrm{d}}\left(\mu_{1}, \mu_{2}\right) \quad \text { as } n \uparrow \infty,
\end{aligned}
$$

where $\mathrm{HK}_{n \mathrm{~d}}$ (resp. $\mathrm{K}_{\mathrm{d} / n}$ ) is the $\mathrm{HK}$ distance induced by $n \mathrm{~d}$ (resp. $\left.\mathrm{d} / n\right)$.

(vi) Combining the superposition principle and the duality with HamiltonJacobi equations, we eventually prove that $\mathrm{H}$ admits an equivalent dynamic characterization "à la Benamou-Brenier" [7,18] (see also the recent [27]) in $X=\mathbb{R}^{d}$ : 


$$
\begin{aligned}
& \mathrm{H}^{2}\left(\mu_{0}, \mu_{1}\right)=\min \left\{\int_{0}^{1} \int\left(\left|\boldsymbol{v}_{t}\right|^{2}+\frac{1}{4}\left|w_{t}\right|^{2}\right) \mathrm{d} \mu_{t} \mathrm{~d} t:\right. \\
& \mu \in \mathrm{C}\left([0,1] ; \mathcal{M}\left(\mathbb{R}^{d}\right)\right), \mu_{t=i}=\mu_{i}, \\
&\left.\partial_{t} \mu_{t}+\nabla \cdot\left(\boldsymbol{v}_{t} \mu_{t}\right)=w_{t} \mu_{t} \text { in } \mathscr{D}^{\prime}\left(\mathbb{R}^{d} \times(0,1)\right)\right\} .
\end{aligned}
$$

Moreover, for the length space $X=\mathbb{R}^{d}$ a curve $[0,1] \ni t \mapsto \mu(t)$ is geodesic curve w.r.t. $\mathrm{K}$ if and only if the coupled system

$$
\partial_{t} \mu_{t}+\nabla \cdot\left(\mathrm{D}_{x} \xi_{t} \mu_{t}\right)=4 \xi_{t} \mu_{t}, \quad \partial_{t} \xi_{t}+\frac{1}{2}\left|\mathrm{D}_{x} \xi^{2}\right|^{2}+2 \xi_{t}^{2}=0
$$

holds for a suitable solution $\xi_{t}=\mathscr{P}_{t} \xi_{0}$. The representation (1.28) is the starting point for further investigations concerning the link to gradient systems and reaction-diffusion equations, the cone geometry, the representation of geodesics and of $\lambda$-convex integral functionals: we refer the interested reader to the examples collected in [30].

Recall that the $\mathrm{HK}$ variational problem is just one example in the realm of Entropy-Transport problems, and we think that other interesting applications can arise by different choices of entropies and cost. One of the simplest variations is to choose the (seemingly more natural) quadratic cost function $\mathrm{c}\left(x_{1}, x_{2}\right):=\mathrm{d}^{2}\left(x_{1}, x_{2}\right)$ instead of the more "exotic" (1.19). The resulting functional is still associated to a distance expressed by

$$
\mathrm{GK}^{2}\left(\mu_{1}, \mu_{2}\right):=\min \left\{\int\left(r_{1}^{2}+r_{2}^{2}-2 r_{1} r_{2} \exp \left(-\mathrm{d}^{2}\left(x_{1}, x_{2}\right) / 2\right)\right) \mathrm{d} \boldsymbol{\alpha}\right\}
$$

where the minimum runs among all the plans $\boldsymbol{\alpha} \in \mathcal{M}(\mathfrak{C} \times \mathfrak{C})$ such that $\mathfrak{h}^{2} \pi_{+}^{i} \boldsymbol{\alpha}=$ $\mu_{i}$ (we propose the name Gaussian Hellinger-Kantorovich distance). If ( $\left.X, \mathrm{~d}\right)$ is a complete, separable and length metric space, $(\mathcal{M}(X), G K)$ is a complete and separable metric space, inducing the weak topology as $\mathrm{HK}$. However, it is not a length space in general, and we will show that the length distance generated by $\mathrm{GK}$ is precisely $\mathrm{HK}$.

The plan of the paper is as follows.

Part I develops the general theory of Optimal Entropy-Transport problems. Section 2 collects some preliminary material, in particular concerning the measure-theoretic setting in arbitrary Hausdorff topological spaces (here we follow [44]) and entropy functionals. We devote some effort to deal with general functionals (allowing a singular part in the Definition (1.3)) in order to include entropies which may have only linear growth. The extension to this 
general framework of the duality Theorem 2.7 (well known in Polish topologies) requires some care and the use of lower semicontinuous test functions instead of continuous ones.

Section 3 introduces the class of Entropy-Transport problems, discussing some examples and proving a general existence result for optimal plans. The "reverse" formulation of Theorem 3.11, though simple, justifies the importance of dealing with the largest class of entropies and will play a crucial role in Sect. 5 .

Section 4 is devoted to finding the dual formulation, proving its equivalence with the primal problem (cf. Theorem 4.11), deriving sharp optimality conditions (cf. Theorem 4.6) and proving the existence of optimal potentials in a suitable generalized sense (cf. Theorem 4.15). The particular class of "regular" problems (where the results are richer) is also studied in some detail.

Section 5 introduces the third formulation (1.12) based on the marginal perspective function (1.11) and its "homogeneous" version (Sect. 5.2). The proof of the equivalence with the previous formulations is presented in Theorem 5.5 and Theorem 5.8. This part provides the crucial link for the further development in the cone setting.

Part II is devoted to Logarithmic Entropy-Transport (LET) problems (Sect. 6) and to their applications to the Hellinger-Kantorovich distance $\mathrm{HK}$ on $\mathcal{N}(X)$.

The Hellinger-Kantorovich distance is introduced by the lifting technique in the cone space in Sect. 7, where we try to follow a presentation modeled on the standard one for the Kantorovich-Wasserstein distance, independently from the results on the LET-problems. After a brief review of the cone geometry (Sect. 7.1) we discuss in some detail the crucial notion of homogeneous marginals in Sect. 7.2 and the useful tightness conditions (Lemma 7.3) for plans with prescribed homogeneous marginals. Section 7.3 introduces the definition of the $\mathrm{H}$ distance and its basic properties. The crucial rescaling and gluing techniques are discussed in Sect. 7.4: they lie at the core of the main metric properties of $\mathrm{K}$, leading to the proof of the triangle inequality and to the characterizations of various metric and topological properties in Sect. 7.5. The equivalence with the LET formulation is the main achievement of Sect. 7.6 (Theorem 7.20), with applications to the duality formula (Theorem 7.21), to the comparisons with the classical Hellinger and Kantorovich distances (Sect. 7.7) and with the Gaussian Hellinger-Kantorovich distance (Sect. 7.8).

The last section of the paper collects various important properties of $\mathrm{HK}$ that share a common "dynamic" flavor. After a preliminary discussion of absolutely continuous curves and geodesics in the cone space $\mathfrak{C}$ in Sect. 8.1, we derive the basic superposition principle in Theorem 8.4. This is the cornerstone to obtain a precise characterization of geodesics (Theorem 8.6), a sharp lower curvature bound in the Alexandrov sense (Theorem 8.8), and to prove the dynamic 
characterization à la Benamou-Brenier of Sect. 8.5. The other powerful tool is provided by the duality with subsolutions to the Hamilton-Jacobi equation (Theorem 8.12), which we derive after a preliminary characterization of metric slopes for a suitable class of test functions in $\mathfrak{C}$. One of the most striking results of Sect. 8.4 is the explicit representation formula for solutions to the HamiltonJacobi equation in $X$, that we obtain by a careful reduction technique from the Hopf-Lax formula in $\mathfrak{C}$. In this respect, we think that Theorem 8.11 is interesting by itself and could find important applications in different contexts. From the point of view of Entropy-Transport problems, Theorem 8.11 is particularly relevant since it provides a dynamic interpretation of the dual characterization of the LFT functional. In Sect. 8.6 we show that in the Euclidean case $X=\mathbb{R}^{d}$ all geodesic curves are characterized by the system (1.29). The last Sect. 8.7 provides various contraction results: in particular we extend the well known contraction property of the Heat flow in spaces with nonnegative Riemannian Ricci curvature to $\mathrm{H}$.

Note during final preparation. The earliest parts of the work developed here were first presented at the ERC Workshop on Optimal Transportation and Applications in Pisa in 2012. Since then the authors developed the theory continuously further and presented results at different workshops and seminars, see Appendix A for some remarks concerning the chronological development of our theory.

In June 2015 the authors became aware of the parallel work [27], which mainly concerns the dynamical approach to the Hellinger-Kantorovich distance discussed in Sect. 8.5 and the metric-topological properties of Sect. 7.5 in the Euclidean case.

Moreover, in mid August 2015 they became aware of the works [13,14], which start from the dynamical formulation of the Hellinger-Kantorovich distance in the Euclidean case, prove existence of geodesics and sufficient optimality and uniqueness conditions (which we state in a stronger form in Sect. 8.6) with a precise characterization in the case of a pair of Dirac masses. Moreover, they provide a detailed discussion of curvature properties following Otto's formalism [36], and study more general dynamic costs on the cone space with their equivalent primal and dual static formulation (leading to characterizations analogous to (7.1) and (6.14) in the Hellinger-Kantorovich case).

Apart from the few above remarks, these independent works did not influence the first (cf. arXiv1508.07941v1) and the present version of this manuscript, which is essentially a minor modification and correction of the first version. In the final Appendix A we give a brief account of the chronological development of our theory. 


\section{Part I. Optimal Entropy-Transport problems}

\section{Preliminaries}

\subsection{Measure theoretic notation}

Positive Radon measures, narrow and weak convergence, tightness. Let $(X, \tau)$ be a Hausdorff topological space. We will denote by $\mathcal{B}(X)$ the $\sigma$-algebra of its Borel sets and by $\mathcal{M}(X)$ the set of finite nonnegative Radon measures on $X$ [44], i.e. $\sigma$-additive set functions $\mu: \mathcal{B}(X) \rightarrow[0, \infty)$ such that

$$
\forall B \in \mathcal{B}(X), \forall \varepsilon>0 \quad \exists K_{\varepsilon} \subset B \text { compact such that } \mu\left(B \backslash K_{\varepsilon}\right) \leq \varepsilon . \text { (2.1) }
$$

The restriction $B \mapsto \mu(B \cap A)$ of a Radon measure $\mu$ to a Borel set $A$ will be denoted by $\mu\llcorner A$.

Radon measures have strong continuity properties with respect to monotone convergence. For this, denote by $\operatorname{LSC}(X)$ the space of all lower semicontinuous real-valued functions on $X$ and consider a nondecreasing directed family $\left(f_{\lambda}\right)_{\lambda \in \mathbb{L}} \subset \operatorname{LSC}(X)$ (where $\mathbb{L}$ is a possibly uncountable directed set) of nonnegative and lower semicontinuous functions $f_{\lambda}$ converging to $f$, we have (cf. [44, Prop. 5, p. 42])

$$
\lim _{\lambda \in \mathbb{L}} \int_{X} f_{\lambda} \mathrm{d} \mu=\int_{X} f \mathrm{~d} \mu \text { for all } \mu \in \mathcal{M}(X) .
$$

We endow $\mathcal{M}(X)$ with the narrow topology, the coarsest (Hausdorff) topology for which all the maps $\mu \mapsto \int_{X} \varphi \mathrm{d} \mu$ are lower semicontinuous, as $\varphi: X \rightarrow \mathbb{R}$ varies among the set $\operatorname{LSC}_{b}(X)$ of all bounded lower semicontinuous functions [44, p. 370, Def. 1].

Remark 2.1 (Radon versus Borel, narrow versus weak) When $(X, \tau)$ is a Radon space (in particular a Polish, or Lusin or Souslin space [44, p. 122]) then every Borel measure satisfies (2.1), so that $\mathcal{M}(X)$ coincides with the set of all nonnegative and finite Borel measures. Narrow topology is in general stronger than the standard weak topology induced by the duality with continuous and bounded functions of $\mathrm{C}_{b}(X)$. However, when $(X, \tau)$ is completely regular, i.e.

$$
\begin{aligned}
& \text { for any closed set } F \subset X \text { and any } x_{0} \in X \backslash F \\
& \text { there exists } f \in C_{b}(X) \text { with } f\left(x_{0}\right)>0 \text { and } f \equiv 0 \text { on } F,
\end{aligned}
$$

(in particular when $\tau$ is metrizable), narrow and weak topology coincide [44, p. 371]. Therefore when $(X, \tau)$ is a Polish space we recover the usual setting of Borel measures endowed with the weak topology. 
We now turn to the compactness properties of subsets of $\mathcal{M}(X)$. Let us first recall that a set $\mathcal{K} \subset \mathcal{M}(X)$ is bounded if $\sup _{\mu \in \mathcal{K}} \mu(X)<\infty$; it is equally tight if

$$
\forall \varepsilon>0 \quad \exists K_{\varepsilon} \subset X \text { compact } \quad \forall \mu \in \mathcal{K}: \mu\left(X \backslash K_{\varepsilon}\right) \leq \varepsilon .
$$

Compactness with respect to narrow topology is guaranteed by an extended version of Prokhorov's Theorem [44, Thm. 3, p. 379]. Tightness of weakly convergent sequences in metrizable spaces is due to LE CAM [29].

Theorem 2.2 If a subset $\mathcal{K} \subset \mathcal{M}(X)$ is bounded and equally tight then it is relatively compact with respect to the narrow topology. The converse is also true in the following cases:

(i) $(X, \tau)$ is a locally compact or a Polish space;

(ii) $(X, \tau)$ is metrizable and $\mathcal{K}=\left\{\mu_{n}: n \in \mathbb{N}\right\}$ for a given weakly convergent sequence $\left(\mu_{n}\right)$.

If $\mu \in \mathcal{M}(X)$ and $Y$ is another Hausdorff topological space, a map $T: X \rightarrow$ $Y$ is Lusin $\mu$-measurable [44, Ch. I, Sect. 5] if for every $\varepsilon>0$ there exists a compact set $K_{\varepsilon} \subset X$ such that $\mu\left(X \backslash K_{\varepsilon}\right) \leq \varepsilon$ and the restriction of $T$ to $K_{\varepsilon}$ is continuous. We denote by $T_{\sharp} \mu \in \mathcal{N}(Y)$ the push-forward measure defined by

$$
T_{\sharp} \mu(B):=\mu\left(T^{-1}(B)\right) \text { for every } B \in \mathcal{B}(Y) \text {. }
$$

For $\mu \in \mathcal{N}(X)$ and a Lusin $\mu$-measurable $T: X \rightarrow Y$, we have $T_{\sharp} \mu \in$ $\mathcal{M}(Y)$. The linear space $\mathrm{B}(X)$ (resp. $\left.\mathrm{B}_{b}(X)\right)$ denotes the space of real Borel (resp. bounded Borel) functions. If $\mu \in \mathcal{M}(X), p \in[1, \infty]$, we will denote by $\mathrm{L}^{p}(X, \mu)$ the subspace of Borel $p$-integrable functions w.r.t. $\mu$, without identifying $\mu$-almost equal functions.

Lebesgue decomposition. Given $\gamma, \mu \in \mathcal{M}(X)$, we write $\gamma \ll \mu$ if $\mu(A)=0$ yields $\gamma(A)=0$ for every $A \in \mathcal{B}(X)$. We say that $\gamma \perp \mu$ if there exists $B \in \mathcal{B}(X)$ such that $\mu(B)=0=\gamma(X \backslash B)$.

Lemma 2.3 (Lebesgue decomposition) For every $\gamma, \mu \in \mathcal{M}(X)$ with $\gamma(X)+$ $\mu(X)>0$ there exists Borel functions $\sigma, \varrho: X \rightarrow[0, \infty)$ and a Borel partition $\left(A, A_{\gamma}, A_{\mu}\right)$ of $X$ with the following properties:

$$
A=\{x \in X: \sigma(x)>0\}=\{x \in X: \varrho(x)>0\}, \quad \sigma \cdot \varrho \equiv 1 \text { in } A,
$$




$$
\begin{aligned}
& \gamma=\sigma \mu+\gamma^{\perp}, \quad \sigma \in \mathrm{L}_{+}^{1}(X, \mu), \quad \gamma^{\perp} \perp \mu, \quad \gamma^{\perp}\left(X \backslash A_{\gamma}\right)=\mu\left(A_{\gamma}\right)=0, \\
& \mu=\varrho \gamma+\mu^{\perp}, \quad \varrho \in \mathrm{L}_{+}^{1}(X, \gamma), \quad \mu^{\perp} \perp \gamma, \quad \mu^{\perp}\left(X \backslash A_{\mu}\right)=\gamma\left(A_{\mu}\right)=0 .
\end{aligned}
$$

Moreover, the sets $A, A_{\gamma}, A_{\mu}$ and the densities $\sigma, \varrho$ are uniquely determined up to $(\mu+\gamma)$-negligible sets.

Proof Let $\theta \in \mathrm{B}(X ;[0,1])$ be the Lebesgue density of $\gamma$ w.r.t. $v:=\mu+\gamma$. Thus, $\theta$ is uniquely determined up to $v$-negligible sets. The Borel partition can be defined by setting $A:=\{x \in X: 0<\theta(x)<1\}, A_{\gamma}:=\{x \in X$ : $\theta(x)=1\}$ and $A_{\mu}:=\{x \in X: \theta(x)=0\}$. By defining $\sigma:=\theta /(1-\theta)$, $\varrho:=1 / \sigma=(1-\theta) / \theta$ for every $x \in A$ and $\sigma=\varrho \equiv 0$ in $X \backslash A$, we obtain Borel functions satisfying (2.7) and (2.8).

Conversely, it is not difficult to check that starting from a decomposition as in (2.6), (2.7), and (2.8) and defining $\theta \equiv 0$ in $A_{\mu}, \theta \equiv 1$ in $A_{\gamma}$ and $\theta:=\sigma /(1+\sigma)$ in $A$ we obtain a Borel function with values in $[0,1]$ such that $\gamma=\theta(\mu+\gamma)$.

\subsection{Min-max and duality}

We recall now a powerful form of von Neumann's Theorem, concerning minimax properties of convex-concave functions in convex subsets of vector spaces and refer to [20, Prop. 1.2+3.2, Chap. VI] for a general exposition.

Let $A, B$ be nonempty convex sets of some vector spaces and let us suppose that $A$ is endowed with a Hausdorff topology. Let $L: A \times B \rightarrow \mathbb{R}$ be a function such that

$a \mapsto L(a, b)$ is convex and lower semicont. in $A$ for every $b \in B$,

$$
b \mapsto L(a, b) \text { is concave in } B \text { for every } a \in A \text {. }
$$

Notice that for arbitrary functions $L$ one always has

$$
\inf _{a \in A} \sup _{b \in B} L(a, b) \geq \sup _{b \in B} \inf _{a \in A} L(a, b) \text {; }
$$

so that equality holds in (2.10) if $\sup _{b \in B} \inf _{a \in A} L(a, b)=+\infty$. When $\sup _{b \in B} \inf _{a \in A} L(a, b)$ is finite, we can still have equality thanks to the following result.

The statement has the advantage of involving a minimal set of topological assumptions (we refer to [45, Thm. 3.1] for the proof; see also [9, Chapter 1, Prop. 1.1]). 
Theorem 2.4 (Minimax duality) Assume that (2.9a) and (2.9b) hold. If there exists $b_{\star} \in B$ and $C>\sup _{b \in B} \inf _{a \in A} L(a, b)$ such that

$$
\left\{a \in A: L\left(a, b_{\star}\right) \leq C\right\} \text { is compact in } A,
$$

then

$$
\inf _{a \in A} \sup _{b \in B} L(a, b)=\sup _{b \in B} \inf _{a \in A} L(a, b) .
$$

\subsection{Entropy functions and their conjugates}

Entropy functions in $[0, \infty)$. We say that $F:[0, \infty) \rightarrow[0, \infty]$ belongs to the class $\Gamma\left(\mathbb{R}_{+}\right)$of admissible entropy function if it satisfies

$F$ is convex and lower semicontinuous and $\mathrm{D}(F) \cap(0, \infty) \neq \emptyset$,

where

$$
\mathrm{D}(F):=\{s \geq 0: F(s)<\infty\} .
$$

It is useful to recall that for every $x_{0} \in \mathrm{D}(F)$ the map $x \mapsto \frac{F(x)-F\left(x_{0}\right)}{x-x_{0}}$ is increasing in $\mathrm{D}(F) \backslash\left\{x_{0}\right\}$, thanks to the convexity of $F$. The recession constant $F_{\infty}^{\prime}$, the right derivative $F_{0}^{\prime}$ at 0 , and the asymptotic affine coefficient aff $F_{\infty}$ are defined by

$$
\begin{aligned}
F_{\infty}^{\prime} & :=\lim _{s \rightarrow \infty} \frac{F(s)}{s}=\sup _{s>0} \frac{F(s)-F\left(s_{o}\right)}{s-s_{o}}, \quad s_{o} \in \mathrm{D}(F) ; \\
F_{0}^{\prime} & := \begin{cases}-\infty & \text { if } F(0)=+\infty, \\
\lim _{s \downarrow 0} \frac{F(s)-F(0)}{s} & \text { otherwise; }\end{cases} \\
\operatorname{aff} F_{\infty} & := \begin{cases}+\infty & \text { if } F_{\infty}^{\prime}=+\infty, \\
\lim _{s \rightarrow \infty}\left(F_{\infty}^{\prime} s-F(s)\right) & \text { otherwise. }\end{cases}
\end{aligned}
$$

To avoid trivial cases, we assumed in (2.13) that the proper domain $\mathrm{D}(F)$ contains at least one strictly positive real number. By convexity, $\mathrm{D}(F)$ is a subinterval of $[0, \infty)$, and we will mainly focus on the case when $\mathrm{D}(F)$ has nonempty interior and $F$ has superlinear growth, i.e. $F_{\infty}^{\prime}=+\infty$. Still it will be useful to deal with the general class defined by (2.13).

Legendre duality. The Legendre conjugate function $F^{*}: \mathbb{R} \rightarrow(-\infty,+\infty]$ is defined by

$$
F^{*}(\phi):=\sup _{s \geq 0}(s \phi-F(s)),
$$


with proper domain $\mathrm{D}\left(F^{*}\right):=\left\{\phi \in \mathbb{R}: F^{*}(\phi) \in \mathbb{R}\right\}$; we will also denote by $\mathrm{D}^{\circ}\left(F^{*}\right)$ the interior of $\mathrm{D}\left(F^{*}\right)$. Strictly speaking, $F^{*}$ is the conjugate of the convex function $\tilde{F}: \mathbb{R} \rightarrow(-\infty,+\infty]$, obtained by extending $F$ to $+\infty$ for negative arguments, and it is related to the subdifferential $\partial F: \mathbb{R} \rightarrow 2^{\mathbb{R}}$ by

$$
\phi \in \partial F(s) \Leftrightarrow s \in \mathrm{D}(F), \quad \phi \in \mathrm{D}\left(F^{*}\right), \quad F(s)+F^{*}(\phi)=s \phi .
$$

Notice that

$$
\inf \mathrm{D}\left(F^{*}\right)=-\infty, \quad \sup \mathrm{D}\left(F^{*}\right)=F_{\infty}^{\prime}, \quad \stackrel{\circ}{\mathrm{D}}\left(F^{*}\right)=\left(-\infty, F_{\infty}^{\prime}\right),
$$

so that $F^{*}$ is finite and continuous in $\left(-\infty, F_{\infty}^{\prime}\right)$, nondecreasing, and satisfies

$$
\lim _{\phi \downarrow-\infty} F^{*}(\phi)=\inf F^{*}=-F(0), \quad \sup F^{*}=\lim _{\phi \uparrow+\infty} F^{*}(\phi)=+\infty .
$$

Concerning the behavior of $F^{*}$ at the boundary of its proper domain we can distinguish a few cases depending on the behavior of $F$ at 0 and $+\infty$ :

- If $F_{0}^{\prime}=-\infty$ (in particular if $F(0)=+\infty$ ) then $F^{*}$ is strictly increasing in $\mathrm{D}\left(F^{*}\right)$.

- If $F_{0}^{\prime}$ is finite, then $F^{*}$ is strictly increasing in $\left[F_{0}^{\prime}, F_{\infty}^{\prime}\right)$ and takes the constant value $-F(0)$ in $\left(-\infty, F_{0}^{\prime}\right]$. Thus $-F(0)$ belongs to the range of $F^{*}$ only if $F_{0}^{\prime}>-\infty$.

- If $F_{\infty}^{\prime}$ is finite, then $\lim _{\phi \uparrow F_{\infty}^{\prime}} F^{*}(\phi)=$ aff $F_{\infty}$. Thus $F_{\infty}^{\prime} \in \mathrm{D}\left(F^{*}\right)$ only if aff $F_{\infty}<\infty$.

- The degenerate case when $F_{\infty}^{\prime}=F_{0}^{\prime}$ occurs only when $F$ is linear.

If $F$ is not linear, we always have

$$
\left.\begin{array}{l}
F^{*} \text { is an increasing homeomorphism } \\
\text { between }\left(F_{0}^{\prime}, F_{\infty}^{\prime}\right) \text { and }\left(-F(0), \text { aff } F_{\infty}\right)
\end{array}\right\}
$$

with the obvious extensions to the boundaries of the intervals when $F_{0}^{\prime}$ or aff $F_{\infty}$ are finite.

We introduce the closed convex subset $\mathfrak{F}$ of $\mathbb{R}^{2}$ associated to the epigraph of $F^{*}$

$$
\begin{aligned}
\mathfrak{F} & :=\left\{(\phi, \psi) \in \mathbb{R}^{2}: \psi \leq-F^{*}(\phi)\right\} \\
& =\left\{(\phi, \psi) \in \mathbb{R}^{2}: s \phi+\psi \leq F(s) \forall s>0\right\}
\end{aligned}
$$

since $\mathrm{D}\left(F^{*}\right)$ has nonempty interior, $\mathfrak{F}$ has nonempty interior $\stackrel{\mathfrak{F}}{\text { as well, with }}$

$$
\stackrel{\circ}{\mathfrak{F}}=\left\{(\phi, \psi) \in \mathbb{R}^{2}: \phi \in \stackrel{\circ}{\mathrm{D}}\left(F^{*}\right), \psi<-F^{*}(\phi)\right\},
$$


and that $\mathfrak{F}=\overline{\mathfrak{F}}$. The function $F$ can be recovered from $F^{*}$ and from $\mathfrak{F}$ through the dual Fenchel-Moreau formula

$$
F(s)=\sup _{\phi \in \mathbb{R}}\left(s \phi-F^{*}(\phi)\right)=\sup _{(\phi, \psi) \in \mathfrak{F}} s \phi+\psi=\sup _{(\phi, \psi) \in \mathfrak{F}} s \phi+\psi .
$$

Notice that $\mathfrak{F}$ satisfies the obvious monotonicity property

$$
(\phi, \psi) \in \stackrel{\circ}{\mathfrak{F}}, \quad \tilde{\psi} \leq \psi, \tilde{\phi} \leq \phi \quad \Rightarrow \quad(\tilde{\phi}, \tilde{\psi}) \in \stackrel{\circ}{\mathfrak{F}}
$$

If $F$ is finite in a neighborhood of $+\infty$, then $F^{*}$ is superlinear as $\phi \uparrow \infty$. More precisely, its asymptotic behavior as $\phi \rightarrow \pm \infty$ is related to the proper domain of $F$ by

$$
s_{F}^{ \pm}=\lim _{\phi \rightarrow \pm \infty} \frac{F^{*}(\phi)}{\phi} .
$$

We will also use the duality formula

$$
(\lambda F(\cdot))^{*}=\lambda F^{*}(\cdot / \lambda) \quad \lambda>0
$$

and we adopt the notation $\phi_{-}$and $\phi_{+}$to denote the negative and the positive part of a function $\phi$, where $\phi_{-}(x):=\min \{\phi(x), 0\}$ and $\phi_{+}(x):=\max \{\phi(x), 0\}$.

Example 2.5 (Power-like entropies) An important class of entropy functions is provided by the power like functions $U_{p}:[0, \infty) \rightarrow[0, \infty]$ with $p \in \mathbb{R}$ characterized by

$$
\begin{gathered}
U_{p} \in \mathrm{C}^{\infty}(0, \infty), \quad U_{p}(1)=U_{p}^{\prime}(1)=0, \\
U_{p}^{\prime \prime}(s)=s^{p-2}, \quad U_{p}(0)=\lim _{s \downarrow 0} U_{p}(s) .
\end{gathered}
$$

Equivalently, we have the explicit formulas

$$
U_{p}(s)=\left\{\begin{array}{ll}
\frac{1}{p(p-1)}\left(s^{p}-p(s-1)-1\right) & \text { if } p \neq 0,1, \\
s \log s-s+1 & \text { if } p=1, \\
s-1-\log s & \text { if } p=0,
\end{array} \quad \text { for } s>0\right.
$$

with $U_{p}(0)=1 / p$ if $p>0$ and $U_{p}(0)=+\infty$ if $p \leq 0$. 
Using the dual exponent $q=p /(p-1)$, the corresponding Legendre conjugates read

$$
U_{q}^{*}(\phi):=\left\{\begin{array}{lll}
\frac{q-1}{q}\left[\left(1+\frac{\phi}{q-1}\right)_{+}^{q}-1\right], & \mathrm{D}\left(U_{q}^{*}\right)=\mathbb{R}, & \text { if } p>1, q>1, \\
\mathrm{e}^{\phi}-1, & \text { if } p=1, q=\infty, \\
\frac{q-1}{q}\left[\left(1+\frac{\phi}{q-1}\right)^{q}-1\right], & \mathrm{D}\left(U_{q}^{*}\right)=(-\infty, 1-q), & \text { if } 0<p<1, q<0, \\
-\log (1-\phi), & \mathrm{D}\left(U_{q}^{*}\right)=(-\infty, 1), & \text { if } p=0, q=0, \\
\frac{q-1}{q}\left[\left(1+\frac{\phi}{q-1}\right)^{q}-1\right], & \mathrm{D}\left(U_{q}^{*}\right)=(-\infty, 1-q], & \text { if } p<0,0<q<1 .
\end{array}\right.
$$

Reverse entropies. Let us now introduce the reverse density function $R$ : $[0, \infty) \rightarrow[0, \infty]$ as

$$
R(r):= \begin{cases}r F(1 / r) & \text { if } r>0 \\ F_{\infty}^{\prime} & \text { if } r=0\end{cases}
$$

It is not difficult to check that $R$ is a proper, convex and lower semicontinuous function, with

$$
R(0)=F_{\infty}^{\prime}, \quad R_{\infty}^{\prime}=F(0), \quad \text { aff } F_{\infty}=-R_{0}^{\prime}, \quad \text { aff } R_{\infty}=-F_{0}^{\prime},
$$

so that $R \in \Gamma\left(\mathbb{R}_{+}\right)$and the map $F \mapsto R$ is an involution on $\Gamma\left(\mathbb{R}_{+}\right)$. A further remarkable involution property is enjoyed by the dual convex set $\mathfrak{R}:=$ $\left\{(\psi, \phi) \in \mathbb{R}^{2}: R^{*}(\psi)+\phi \leq 0\right\}$ defined as $(2.21)$ : it is easy to check that

$$
(\phi, \psi) \in \mathfrak{F} \quad \Leftrightarrow \quad(\psi, \phi) \in \mathfrak{R},
$$

a relation that obviously holds for the interiors of $\mathfrak{F}$ and $\mathfrak{R}$ as well. It follows that the Legendre transform of $R$ and $F$ are related by

$\psi \leq-F^{*}(\phi) \Leftrightarrow \phi \leq-R^{*}(\psi) \Leftrightarrow(\phi, \psi) \in \mathfrak{F}$ for every $\phi, \psi \in \mathbb{R}$,

and, recalling (2.22),

$$
\phi \in \stackrel{\mathrm{D}}{ }\left(F^{*}\right), \psi<-F^{*}(\phi) \Leftrightarrow \psi \in \stackrel{\mathrm{D}}{\mathrm{D}}\left(R^{*}\right), \phi<-R^{*}(\psi) .
$$

Both the above conditions characterize the interior of $\mathfrak{F}$. As in (2.20) we have

$$
\left.\begin{array}{l}
R^{*} \text { is an increasing homeomorphism } \\
\text { between }\left(-\operatorname{aff} F_{\infty}, F(0)\right) \text { and }\left(-F_{\infty}^{\prime},-F_{0}^{\prime}\right)
\end{array}\right\}
$$


with $\mathrm{D}\left(R^{*}\right)=(-\infty, F(0))$. A last useful identity involves the subdifferentials of $F$ and $R$ : for every $s, r>0$ with $s r=1$, and $\phi, \psi \in \mathbb{R}$ we have

$$
\left(\phi \in \partial F(r) \text { and } \psi=-F^{*}(\phi)\right) \Longleftrightarrow\left(\psi \in \partial R(s) \text { and } \phi=-R^{*}(\psi)\right) \text {. }
$$

It is not difficult to check that the reverse entropy associated to $U_{p}$ is $U_{1-p}$.

\subsection{Relative entropy integral functionals}

For $F \in \Gamma\left(\mathbb{R}_{+}\right)$we consider the functional $\mathscr{F}: \mathcal{M}(X) \times \mathcal{M}(X) \rightarrow[0, \infty]$ defined by

$$
\mathscr{F}(\gamma \mid \mu):=\int_{X} F(\sigma) \mathrm{d} \mu+F_{\infty}^{\prime} \gamma^{\perp}(X), \gamma=\sigma \mu+\gamma^{\perp},
$$

where $\gamma=\sigma \mu+\gamma^{\perp}$ is the Lebesgue decomposition of $\gamma$ w.r.t. $\mu$, see Lemma 2.3. Notice that

$$
\text { if } F \text { is superlinear then } \mathscr{F}(\gamma \mid \mu)=+\infty \text { if } \gamma \ll \mu \text {, }
$$

and, whenever $\eta_{0}$ is the null measure, we have

$$
\mathscr{F}\left(\gamma \mid \eta_{0}\right)=F_{\infty}^{\prime} \gamma(X)
$$

where, as usual in measure theory, we adopt the convention $0 \cdot \infty=0$.

Because of our applications in Sect. 3, our next lemma deals with Borel functions $\varphi \in \mathrm{B}(X ; \overline{\mathbb{R}})$ taking values in the extended real line $\overline{\mathbb{R}}:=\mathbb{R} \cup\{ \pm \infty\}$. By $\overline{\mathfrak{F}}$ we denote the closure of $\mathfrak{F}$ in $\overline{\mathbb{R}} \times \overline{\mathbb{R}}$, i.e.

$$
(\phi, \psi) \in \overline{\mathfrak{F}} \Leftrightarrow \begin{cases}\psi \leq-F^{*}(\phi) & \text { if }-\infty<\phi \leq F_{\infty}^{\prime}, \phi<+\infty \\ \psi=-\infty & \text { if } \phi=F_{\infty}^{\prime}=+\infty, \\ \psi \in[-\infty, F(0)] & \text { if } \phi=-\infty,\end{cases}
$$

and, symmetrically by (2.29) and (2.30),

$$
(\phi, \psi) \in \overline{\mathfrak{F}} \Leftrightarrow \begin{cases}\phi \leq-R^{*}(\psi) & \text { if }-\infty<\psi \leq F(0), \psi<+\infty \\ \phi=-\infty & \text { if } \psi=F(0)=+\infty \\ \phi \in\left[-\infty, F_{\infty}^{\prime}\right] & \text { if } \psi=-\infty\end{cases}
$$

In particular, we have

$$
(\phi, \psi) \in \overline{\mathfrak{F}} \Longrightarrow\left(\phi \leq F_{\infty}^{\prime} \text { and } \psi \leq F(0)\right) .
$$


Lemma 2.6 If $\gamma, \mu \in \mathcal{M}(X)$ and $(\phi, \psi) \in \mathrm{B}(X ; \overline{\mathfrak{F}})$ satisfy

$$
\mathscr{F}(\gamma \mid \mu)<\infty, \quad \psi_{-} \in \mathrm{L}^{1}(X, \mu) \quad\left(\text { resp. } \phi_{-} \in \mathrm{L}^{1}(X, \gamma)\right),
$$

then $\phi_{+} \in \mathrm{L}^{1}(X, \gamma)$ (resp. $\left.\psi_{+} \in \mathrm{L}^{1}(X, \mu)\right)$ and

$$
\mathscr{F}(\gamma \mid \mu)-\int_{X} \psi \mathrm{d} \mu \geq \int_{X} \phi \mathrm{d} \gamma .
$$

Whenever $\psi \in \mathrm{L}^{1}(X, \mu)$ or $\phi \in \mathrm{L}^{1}(X, \gamma)$, then equality holds in (2.41) if and only if for the Lebesgue decomposition given by Lemma 2.3 one has

$$
\begin{aligned}
& \phi \in \partial F(\sigma), \psi=-F^{*}(\phi) \quad(\mu+\gamma) \text {-a.e. in } A, \\
& \psi=F(0)<\infty \mu^{\perp} \text {-a.e. in } A_{\mu}, \quad \phi=F_{\infty}^{\prime}<\infty \gamma^{\perp} \text {-a.e. in } A_{\gamma} .
\end{aligned}
$$

Equation (2.42) can equivalently be formulated as $\psi \in \partial R(\varrho)$ and $\phi=$ $-R^{*}(\psi)$.

Proof Let us first show that in both cases the two integrals of (2.41) are well defined (possibly taking the value $-\infty$ ). If $\psi_{-} \in \mathrm{L}^{1}(X, \mu)$ (in particular $\psi>-\infty \mu$-a.e.) with $(\phi, \psi) \in \overline{\mathfrak{F}}$ we use the pointwise bound $s \phi \leq F(s)-\psi$ that yields $s \phi_{+} \leq(F(s)-\psi)_{+} \leq F(s)+\psi_{-}$obtaining $\phi_{+} \in \mathrm{L}^{1}(X, \gamma)$, since $(\phi, \psi) \in \overline{\mathfrak{F}}$ yields $\phi_{+} \leq F_{\infty}^{\prime}$.

If $\phi_{-} \in \mathrm{L}^{1}(X, \gamma)$ (and thus $\phi>-\infty \gamma$-a.e.) the analogous inequality $\psi_{+} \leq F(s)+s \phi_{-}$yields $\psi_{+} \in \mathrm{L}^{1}(X, \mu)$. Then, (2.41) follows from (2.21) and (2.40).

Once $\phi \in \mathrm{L}^{1}(X, \mu)$ (or $\psi \in \mathrm{L}^{1}(X, \gamma)$ ), estimate (2.41) can be written as

$$
\int_{A}(F(\sigma)-\sigma \phi-\psi) \mathrm{d} \mu+\int_{A_{\mu}}(F(0)-\psi) \mathrm{d} \mu^{\perp}+\int_{A_{\gamma}}\left(F_{\infty}^{\prime}-\phi\right) \mathrm{d} \gamma^{\perp} \geq 0
$$

and by (2.21) and (2.40) the equality case immediately yields that each of the three integrals of the previous formula vanishes. Since $(\phi, \psi)$ lies in $\overline{\mathfrak{F}} \subset \mathbb{R}^{2}$ $(\mu+\gamma)$-a.e. in $A$, the vanishing of the first integrand yields $\psi=-F^{*}(\sigma)$ and $\phi \in \partial F(\sigma)$ by (2.17) for $\mu$ and $(\mu+\gamma)$ almost every point in $A$. The equivalence (2.34) provides the reversed identities $\psi \in \partial R(\varrho), \phi=-R^{*}(\psi)$.

The relations in (2.43) follow easily by the vanishing of the last two integrals and the fact that $\psi$ is finite $\mu$-a.e. and $\phi$ is finite $\gamma$-a.e.

A simple application of (2.41) yields the following variant of Jensen's inequality

$$
\mathscr{F}(\gamma \mid \mu) \geq \mu(X) F(\gamma(X) / \mu(X)) \text { whenever } \mu(X)>0 .
$$


In order to prove it, we first choose arbitrary $(\bar{\phi}, \bar{\psi}) \in \mathfrak{F}$ and constant functions $\phi(x) \equiv \bar{\phi}, \psi(x) \equiv \bar{\psi}$ in (2.41), obtaining

$$
\mathscr{F}(\gamma \mid \mu) \geq \mu(X)\left[\bar{\psi}+\frac{\gamma(X)}{\mu(X)} \bar{\phi}\right]
$$

we then take the supremum with respect to $(\bar{\phi}, \bar{\psi}) \in \mathfrak{F}$, recalling (2.23).

The next theorem gives a characterization of the relative entropy $\mathscr{F}$, which is the main result of this section. Its proof is a careful adaptation of [2, Lemma 9.4.4] to the present more general setting, which includes the sublinear case when $F_{\infty}^{\prime}<\infty$ and the lack of complete regularity of the space. This suggests to deal with lower semicontinuous functions instead of continuous ones. Whenever $A \subset \mathbb{R}$, we denote by $\operatorname{LSC}_{S}(X ; A)$ the class of lower semicontinuous simple real functions

$$
\operatorname{LSC}_{s}(X):=\{\varphi: X \rightarrow \mathbb{R}: \varphi \in \operatorname{LSC}(X), \varphi(X) \text { is a finite subset of } A\}
$$

by omitting $A$ when $A=\mathbb{R}$; we introduce the notation $\varphi=-\phi$ and the concave increasing function

$$
F^{\circ}(\varphi):=-F^{*}(-\varphi), \quad F^{\circ}(\varphi)=\inf _{s \geq 0}(\varphi s+F(s)) ;
$$

by (2.18) and (2.19) the interior of the proper domain of $F^{\circ}$ is $\mathrm{D}^{\circ}\left(F^{\circ}\right)=$ $\left(-F_{\infty}^{\prime},+\infty\right)$ and $\lim _{\varphi \downarrow-F_{\infty}^{\prime}} F^{\circ}(\varphi)=-\infty, \lim _{\varphi \uparrow+\infty} F^{\circ}(\varphi)=F(0)$.

Theorem 2.7 (Duality and lower semicontinuity) For every $\gamma, \mu \in \mathcal{M}(X)$ we have

$$
\begin{aligned}
\mathscr{F}(\gamma \mid \mu) & =\sup \left\{\int_{X} \psi \mathrm{d} \mu+\int_{X} \phi \mathrm{d} \gamma: \phi, \psi \in \operatorname{LSC}_{S}(X),\right. \\
& (\phi(x), \psi(x)) \in \stackrel{\mathfrak{F}}{ } \forall x \in X\} \\
& =\sup \left\{\int_{X} \psi \mathrm{d} \mu-\int_{X} R^{*}(\psi) \mathrm{d} \gamma: \psi \in \operatorname{LSC}_{S}\left(X, \stackrel{\circ}{\mathrm{D}}\left(R^{*}\right)\right)\right\} \\
& =\sup \left\{\int_{X} F^{\circ}(\varphi) \mathrm{d} \mu-\int_{X} \varphi \mathrm{d} \gamma: \varphi \in \operatorname{LSC}_{S}\left(X, \stackrel{\circ}{\mathrm{D}}\left(F^{\circ}\right)\right)\right\} .
\end{aligned}
$$

Moreover, the space $\operatorname{LSC}_{S}(X)$ in the supremum of (2.46), can also be replaced by the space $\mathrm{LSC}_{b}(X)$ of bounded l.s.c. functions or by the space $\mathrm{B}_{b}(X)$ of bounded Borel functions and the constraint $(\phi(x), \psi(x)) \in \mathfrak{F}$ in (2.46) can also be relaxed to $(\phi(x), \psi(x)) \in \mathfrak{F}$ for every $x \in X$. Similarly, the 
spaces $\operatorname{LSC}_{S}\left(X, \mathrm{D}^{*}\left(R^{*}\right)\right)\left(\right.$ resp. $\left.\operatorname{LSC}_{S}\left(X, \stackrel{\mathrm{D}}{ }\left(F^{\circ}\right)\right)\right)$ of (2.47) (resp. (2.48)) can be replaced by $\operatorname{LSC}_{b}\left(X, \mathrm{D}\left(R^{*}\right)\right)$ or $\mathrm{B}_{b}\left(X, \mathrm{D}\left(R^{*}\right)\right)$ (resp. $\operatorname{LSC}_{b}\left(X, \mathrm{D}\left(F^{\circ}\right)\right)$ or $\mathrm{B}_{b}\left(X, \mathrm{D}\left(F^{\circ}\right)\right)$ ).

Remark 2.8 If $(X, \tau)$ is completely regular (recall (2.3)), then we can equivalently replace lower semicontinuous functions by continuous ones in (2.46), (2.47) and (2.48). E.g. in the case of (2.46) we have

$$
\mathscr{F}(\gamma \mid \mu)=\sup \left\{\int_{X} \psi \mathrm{d} \mu+\int_{X} \phi \mathrm{d} \gamma:(\phi, \psi) \in \mathrm{C}_{b}(X ; \stackrel{\mathfrak{F}}{)})\right\},
$$

whereas (2.47) and (2.48) become

$$
\begin{aligned}
\mathscr{F}(\gamma \mid \mu) & =\sup \left\{\int_{X} \psi \mathrm{d} \mu-\int_{X} R^{*}(\psi) \mathrm{d} \gamma: \psi, R^{*}(\psi) \in \mathrm{C}_{b}(X)\right\} \\
& =\sup \left\{\int_{X} F^{\circ}(\varphi) \mathrm{d} \mu-\int_{X} \varphi \mathrm{d} \gamma: \varphi, F^{\circ}(\varphi) \in \mathrm{C}_{b}(X)\right\} .
\end{aligned}
$$

In fact, considering first (2.46), by complete regularity it is possible to express every pair $\phi, \psi$ of bounded lower semicontinuous functions with values in $\mathfrak{F}$ as the supremum of a directed family of continuous and bounded functions $\left(\phi_{\alpha}, \psi_{\alpha}\right)_{\alpha \in \mathbb{A}}$ which still satisfy the constraint given by $\stackrel{\mathfrak{F}}{\text { due }}$ to $(2.24)$. We can then apply the continuity (2.2) of the integrals with respect to the Radon measures $\mu$ and $\gamma$.

In order to replace 1.s.c. functions with continuous ones in (2.47) we can approximate $\psi$ by an increasing directed family of continuous functions $\left(\psi_{\alpha}\right)_{\alpha \in \mathbb{A}}$. By truncation, one can always assume that $\max \psi \geq \sup \psi_{\alpha} \geq$ $\inf \psi_{\alpha} \geq \min \psi$. Since $R^{*}(\psi)$ is bounded, it is easy to check that also $R^{*}\left(\psi_{\alpha}\right)$ is bounded and it is an increasing directed family converging to $R^{*}(\psi)$. An analogous argument works for (2.49).

Proof Since the statements are trivial in the case when $\mu=\gamma=\eta_{0}$ are the null measure, it is clearly not restrictive to assume $(\mu+\gamma)(X)>0$. Let us prove (2.46): denoting by $\mathscr{F}^{\prime}$ its right-hand side, Lemma 2.6 yields $\mathscr{F} \geq \mathscr{F}^{\prime}$. In order to prove the opposite inequality we consider the Lebesgue decomposition given by Lemma 2.3: let $A_{\gamma} \in \mathcal{B}(X)$ be a $\mu$-negligible Borel set where $\gamma^{\perp}$ is concentrated, let $\tilde{A}:=X \backslash A_{\gamma}=A \cup A_{\mu}$ and let $\sigma: X \rightarrow[0, \infty)$ be a Borel density for $\gamma$ w.r.t. $\mu$. We consider a countable subset $\left(\phi_{n}, \psi_{n}\right)_{n=1}^{\infty}$ with $\psi_{1}=\phi_{1}=0$, which is dense in $\stackrel{\circ}{\mathfrak{F}}$ and an increasing sequence $\bar{\phi}_{n} \in\left(-\infty, F_{\infty}^{\prime}\right)$ converging to $F_{\infty}^{\prime}$, with $\bar{\psi}_{n}:=-F^{*}\left(\bar{\phi}_{n}\right)$. By (2.23) we have

$$
F(\sigma(x))=\lim _{N \uparrow \infty} F_{N}(x) \text {, where } \forall x \in X: F_{N}(x):=\sup _{1 \leq n \leq N} \psi_{n}+\sigma(x) \phi_{n} .
$$


Hence, Beppo Levi's monotone convergence theorem (notice that $F_{N} \geq F_{1}=$ $0)$ implies $\mathscr{F}(\gamma \mid \mu)=\lim _{N \uparrow \infty} \mathscr{F}_{N}^{\prime}(\gamma \mid \mu)$, where

$$
\mathscr{F}_{N}^{\prime}(\gamma \mid \mu):=\int_{\tilde{A}} F_{N}(x) \mathrm{d} \mu(x)+\bar{\phi}_{N} \gamma\left(A_{\gamma}\right)
$$

It is therefore sufficient to prove that

$$
\mathscr{F}^{\prime}(\gamma \mid \mu) \geq \mathscr{F}_{N}^{\prime}(\gamma \mid \mu) \text { for every } N \in \mathbb{N}
$$

We fix $N \in \mathbb{N}$, set $\phi_{0}:=\bar{\phi}_{N}, \psi_{0}:=\bar{\psi}_{N}$, and recursively define the Borel sets $A_{j}$, for $j=0, \ldots, N$, with $A_{0}:=A_{\gamma}$ and

$$
\begin{aligned}
& A_{1}:=\left\{x \in \tilde{A}: F_{1}(x)=F_{N}(x)\right\}, \\
& A_{j}:=\left\{x \in \tilde{A}: F_{N}(x)=F_{j}(x)>F_{j-1}(x)\right\} \text { for } j=2, \ldots, N .
\end{aligned}
$$

Since $F_{1} \leq F_{2} \leq \cdots \leq F_{N}$, the sets $A_{i}, i=1, \ldots, N$, form a Borel partition of $\tilde{A}$. As $\mu$ and $\gamma$ are Radon measures, for every $\varepsilon>0$ we find disjoint compact sets $K_{j} \subset A_{j}$ and disjoint open sets (by the Hausdorff separation property of $X) G_{j} \supset K_{j}$ such that

$$
\sum_{j=0}^{N}\left(\mu\left(A_{j} \backslash K_{j}\right)+\gamma\left(A_{j} \backslash K_{j}\right)\right)=\mu\left(X \backslash \bigcup_{j=0}^{N} K_{j}\right)+\gamma\left(X \backslash \bigcup_{j=0}^{N} K_{j}\right) \leq \varepsilon / S_{N}
$$

where

$$
\begin{aligned}
S_{N} & :=\max _{0 \leq n \leq N}\left[\left(\phi_{n}-\phi_{\min }^{N}\right)+\left(\psi_{n}-\psi_{\min }^{N}\right)\right], \\
\phi_{\min }^{N} & :=\min _{0 \leq j \leq N} \phi_{j}, \quad \psi_{\min }^{N}:=\min _{0 \leq j \leq N} \psi_{j} .
\end{aligned}
$$

Since $\left(\phi_{n}, \psi_{n}\right) \in \mathfrak{F}$ for every $n \in \mathbb{N}$ and $\mathfrak{F}$ satisfies the monotonicity property

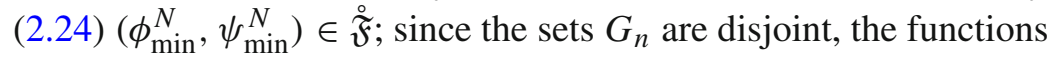

$$
\begin{aligned}
\psi_{N}(x) & := \begin{cases}\psi_{n} & \text { if } x \in G_{n}, \\
\psi_{\min }^{N} & \text { if } x \in X \backslash\left(\cup_{n=1}^{N} G_{n}\right)\end{cases} \\
\phi_{N}(x) & := \begin{cases}\phi_{n} & \text { if } x \in G_{n}, \\
\phi_{\min }^{N} & \text { if } x \in X \backslash\left(\cup_{n=1}^{N} G_{n}\right)\end{cases}
\end{aligned}
$$


take values in $\stackrel{\mathfrak{F}}{\text { and }}$ are lower semicontinuous thanks to the representation formula

$$
\begin{aligned}
& \psi_{N}(x)=\psi_{\min }^{N}+\sum_{n=0}^{N}\left(\psi_{n}-\psi_{\min }^{N}\right) \chi_{G_{n}}(x), \\
& \phi_{N}(x)=\phi_{\min }^{N}+\sum_{n=0}^{N}\left(\phi_{n}-\phi_{\min }^{N}\right) \chi_{G_{n}}(x) .
\end{aligned}
$$

Moreover, they satisfy

$$
\begin{aligned}
\mathscr{F}_{N}^{\prime}(\gamma \mid \mu)= & \sum_{j=1}^{N} \int_{A_{j}} F_{j} \mathrm{~d} \mu+\phi_{0} \gamma\left(A_{0}\right) \\
= & \phi_{\min }^{N} \gamma(X)+\psi_{\min }^{N} \mu(X) \\
& +\sum_{j=0}^{N}\left(\int_{A_{j}}\left(\phi_{j}-\phi_{\min }^{N}\right) \mathrm{d} \gamma+\int_{A_{j}}\left(\psi_{j}-\psi_{\min }^{N}\right) \mathrm{d} \mu\right) \\
\leq & \phi_{\min }^{N} \gamma(X)+\psi_{\min }^{N} \mu(X) \\
& +\sum_{j=0}^{N}\left(\int_{K_{j}}\left(\phi_{j}-\phi_{\min }^{N}\right) \mathrm{d} \gamma+\int_{K_{j}}\left(\psi_{j}-\psi_{\min }^{N}\right) \mathrm{d} \mu\right)+\varepsilon \\
\leq & \int_{X} \phi_{N} \mathrm{~d} \gamma+\int_{X} \psi_{N} \mathrm{~d} \mu+\varepsilon .
\end{aligned}
$$

Since $\varepsilon$ is arbitrary we obtain (2.50).

Equation (2.47) follows directly by (2.46) and the previous Lemma 2.6. In fact, denoting by $\mathscr{F}^{\prime \prime}$ the right-hand side of (2.47), Lemma 2.6 shows that $\mathscr{F}^{\prime \prime}(\gamma \mid \mu) \leq \mathscr{F}(\gamma \mid \mu)=\mathscr{F}^{\prime}(\gamma \mid \mu)$. On the other hand, if $\phi, \psi \in \operatorname{LSC}_{s}(X)$ with $(\phi, \psi) \in \mathfrak{F}$ then $\psi$ takes values in $\stackrel{\circ}{\mathrm{D}}\left(R^{*}\right)$ and $-R^{*}(\psi) \geq \phi$. Hence, the map $x \mapsto R^{*}(\psi(x))$ belongs to $\operatorname{LSC}_{S}(X)$ since $R^{*}$ is real valued and nondecreasing in the interior of its domain, and it is bounded from above by $-\phi$. We thus get $\mathscr{F}^{\prime \prime}(\gamma \mid \mu) \geq \mathscr{F}^{\prime}(\gamma \mid \mu)$.

In order to show (2.48), we observe that for every $\psi \in \operatorname{LSC}_{S}\left(X, \mathrm{D}\left(R^{*}\right)\right)$ and $\varepsilon>0$ we can set $\varphi:=R^{*}(\psi)+\varepsilon \in \operatorname{LSC}_{S}\left(X, \stackrel{\circ}{\mathrm{D}}\left(F^{\circ}\right)\right)$; since $\left(\psi,-R^{*}(\psi)-\right.$ $\varepsilon) \in \stackrel{\circ}{R},(2.31)$ yields $\psi<-F^{*}(-\varphi)=F^{\circ}(\varphi)$ so that $\int F^{\circ}(\varphi) \mathrm{d} \mu-\int \varphi \mathrm{d} \gamma \geq$ $\int \psi \mathrm{d} \mu-\int R^{*}(\psi) \mathrm{d} \gamma-\varepsilon \gamma(X)$. By construction and (2.30) we also have 
$\left(-\varphi, F^{\circ}(\varphi)\right) \in \mathfrak{F}$ so that $\int F^{\circ}(\varphi) \mathrm{d} \mu-\int \varphi \mathrm{d} \gamma \leq \mathscr{F}(\gamma \mid \mu)$ by Lemma 2.6. Passing to the limit as $\varepsilon \downarrow 0$ and recalling (2.47) we obtain (2.48).

When one replaces $\operatorname{LSC}_{s}(X)$ with $\operatorname{LSC}_{b}(X)$ or $\mathrm{B}_{b}(X)$ in (2.46) and the constraint $(\phi(x), \psi(x)) \in \mathfrak{\mathfrak { F }}$ with $(\phi(x), \psi(x)) \in \mathfrak{F}$ (or even $\overline{\mathfrak{F}}$ ), the supremum is taken on a larger set, so that the right-hand side of (2.46) cannot decrease. On the other hand, Lemma 2.6 shows that $\mathscr{F}(\gamma \mid \mu)$ still provides an upper bound even if $\phi, \psi$ are in $\mathrm{B}_{b}(X)$; thus duality also holds in this case. The same argument applies to (2.47) or (2.48).

The following result provides lower semicontinuity of the relative entropy or of an increasing sequence of relative entropies.

Corollary 2.9 The functional $\mathscr{F}$ is jointly convex and lower semicontinuous in $\mathcal{M}(X) \times \mathcal{M}(X)$. More generally, if $F \in \Gamma\left(\mathbb{R}_{+}\right)$is the pointwise limit of an increasing net $\left(F_{\lambda}\right)_{\lambda \in \mathbb{L}} \subset \Gamma\left(\mathbb{R}_{+}\right)$indexed by a directed set $\mathbb{L}$ and $(\mu, \gamma) \in$ $\mathcal{M}(X) \times \mathcal{M}(X)$ is the narrow limit of a net $\left(\mu_{\lambda}, \gamma_{\lambda}\right)_{\lambda \in \mathbb{L}} \subset \mathcal{M}(X) \times \mathcal{M}(X)$, then the corresponding entropy functionals $\mathscr{F} \lambda, \mathscr{F}$ satisfy

$$
\liminf _{\lambda \in \mathbb{L}} \mathscr{F}_{\lambda}\left(\gamma_{\lambda} \mid \mu_{\lambda}\right) \geq \mathscr{F}(\gamma \mid \mu)
$$

Proof The lower semicontinuity of $\mathscr{F}$ follows by (2.46), which provides a representation of $\mathscr{F}$ as the supremum of a family of lower semicontinuous functionals for the narrow topology. Using $F_{\alpha} \leq F_{\lambda}$ for $\alpha \leq \lambda$ in $\mathbb{L}, \alpha$ fixed, we have

$$
\liminf _{\lambda \in \mathbb{L}} \mathscr{F}_{\lambda}\left(\gamma_{\lambda} \mid \mu_{\lambda}\right) \geq \liminf _{\lambda \in \mathbb{L}} \mathscr{F}_{\alpha}\left(\gamma_{\lambda} \mid \mu_{\lambda}\right) \geq \mathscr{F}_{\alpha}(\gamma \mid \mu),
$$

by the above lower semicontinuity. Hence, it suffices to check that

$$
\lim _{\lambda \in \mathbb{L}} \mathscr{F}_{\lambda}(\gamma \mid \mu)=\mathscr{F}(\gamma \mid \mu) \text { for every } \gamma, \mu \in \mathcal{M}(X) .
$$

This formula follows by the monotonicity of the convex sets $\mathfrak{F}_{\lambda}$ (associated to $F_{\lambda}$ by (2.21)), i.e. $\mathfrak{F}_{\alpha} \subset \mathfrak{F}_{\lambda}$ if $\alpha \leq \lambda$ in $\mathbb{L}$, and by the fact that $\stackrel{\mathfrak{F}}{ } \subset \cup_{\lambda \in \mathbb{L}} \mathfrak{F}_{\lambda}$; in order to show the latter property, we argue by contradiction and we suppose that there exists $(\phi, \psi) \in \mathfrak{\mathfrak { F }}$ which does not belong to $\mathfrak{F}^{\prime}:=\cup_{\lambda \in \mathbb{L}} \mathfrak{F}_{\lambda}^{\circ}$. Notice that every $\mathfrak{F}_{\lambda}$ has nonempty interior, so that $\mathfrak{F}^{\prime}$ is a nonempty convex and open set. We also notice that $\phi<F_{\infty}^{\prime}$ and $\lim _{\lambda \in \mathbb{L}}\left(F_{\lambda}\right)_{\infty}^{\prime}=F_{\infty}^{\prime}$ so that there exists $\alpha \in \mathbb{L}$ with $F_{\alpha}^{*}(\phi)<\infty$; thus there exists $-\bar{\psi}>\psi$ such that $(\phi, \bar{\psi}) \in \mathfrak{F}^{\prime}$. Applying the geometric form of the Hahn-Banach theorem, we can find a non vertical line separating $(\phi, \psi)$ from $\mathfrak{F}^{\prime}$, i.e. there exists $\theta \in \mathbb{R}$ such that

$$
-\psi^{\prime} \geq-\psi+\theta\left(\phi^{\prime}-\phi\right) \text { for every }\left(\phi^{\prime}, \psi^{\prime}\right) \in \mathfrak{F}^{\prime} .
$$


Recalling that $\overline{\check{F}_{\lambda}}=\mathfrak{F}_{\lambda}$ we deduce

$$
F_{\lambda}^{*}\left(\phi^{\prime}\right) \geq-\psi+\theta\left(\phi^{\prime}-\phi\right) \text { for every } \phi^{\prime} \in \mathrm{D}\left(F_{\lambda}^{*}\right), \lambda \in \mathbb{L}
$$

taking the supremum w.r.t. $\phi^{\prime}$ we obtain

$$
\psi+\theta \phi \geq F_{\lambda}(\theta) \text { for every } \lambda \in \mathbb{L}
$$

and passing to the limit w.r.t. $\lambda \in \mathbb{L}$ we get

$$
\psi+\theta \phi \geq F(\theta) \text { so that }-\psi \leq \theta \phi-F(\phi) \leq F^{*}(\phi),
$$

which contradicts the fact that $(\phi, \psi) \in$ int $\mathfrak{F}$.

Thus for every pair of simple and lower semicontinuous functions $(\phi, \psi)$ taking values in int $\mathfrak{F}$ we have $(\psi(x), \phi(x)) \in$ int $\mathfrak{F}_{\alpha}$ for every $x \in X$ and some $\alpha \in \mathbb{L}$ so that

$$
\liminf _{\lambda \in \mathbb{L}} \mathscr{F}_{\lambda}(\gamma \mid \mu) \geq \mathscr{F}_{\alpha}(\gamma \mid \mu) \geq \int_{X} \psi \mathrm{d} \mu+\int_{X} \phi \mathrm{d} \gamma
$$

Since $\phi, \psi$ are arbitrary we conclude applying the duality formula (2.46).

Next, we provide a compactness result for the sublevels of the relative entropy, which will be useful in Sect. 3.4 (see Theorem 3.3 and Lemma 3.9).

Proposition 2.10 (Boundedness and tightness) If $\mathcal{K} \subset \mathcal{M}(X)$ is bounded and $F_{\infty}^{\prime}>0$, then for every $C \geq 0$ the sublevels of $\mathscr{F}$,

$$
\Xi_{C}:=\{\gamma \in \mathcal{M}(X): \mathscr{F}(\gamma \mid \mu) \leq C \text { for some } \mu \in \mathcal{K}\}
$$

are bounded. If moreover $\mathcal{K}$ is equally tight and $F_{\infty}^{\prime}=+\infty$, then the sets $\Xi_{C}$ are equally tight.

Proof Concerning the properties of $\Xi_{C}$, we will use the inequality $\lambda \gamma(B) \leq \mathscr{F}(\gamma \mid \mu)+F^{*}(\lambda) \mu(B)$ for every $\lambda \in\left(0, F_{\infty}^{\prime}\right)$, and $B \in \mathcal{B}(X)$

This follows easily by considering the decomposition $\gamma=\sigma \mu+\gamma^{\perp}$ and by integrating the Young inequality $\lambda \sigma \leq F(\sigma)+F^{*}(\lambda)$ for $\lambda>0$ in $B$ with respect to $\mu$; notice that

$$
\lambda \gamma(B)=\lambda \int_{B} \sigma \mathrm{d} \mu+\lambda \gamma^{\perp}(B) \leq \lambda \int_{B} \sigma \mathrm{d} \mu+F_{\infty}^{\prime} \gamma^{\perp}(B) \text { if } 0<\lambda<F_{\infty}^{\prime}
$$


Choosing first $B=X$ in (2.56) and an arbitrary $\lambda$ in $\left(0, F_{\infty}^{\prime}\right)$ (notice that $F^{*}(\lambda)<\infty$ thanks to (2.18)) we immediately get a uniform bound of $\gamma(X)$ for every $\gamma \in \Xi_{C}$.

In order to prove the tightness when $F_{\infty}^{\prime}=+\infty$, whenever $\varepsilon>0$ is given, we can choose $\lambda=2 C / \varepsilon$ and $\eta>0$ so small that $\eta F^{*}(\lambda) / \lambda \leq \varepsilon / 2$, and then a compact set $K \subset X$ such that $\mu(X \backslash K) \leq \eta$ for every $\mu \in \mathcal{K}$. (2.56) shows that $\gamma(X \backslash K) \leq \varepsilon$ for every $\gamma \in \Xi$.

We conclude this section with a useful representation of $\mathscr{F}$ in terms of the reverse entropy $R$ (2.28) and the corresponding functional $\mathscr{R}$. We will use the result in Sect. 3.5 for the reverse formulation of the primal entropy-transport problem.

Lemma 2.11 For every $\gamma, \mu \in \mathcal{M}(X)$ we define

$$
\mathscr{R}(\mu \mid \gamma)=\int_{X} R(\varrho) \mathrm{d} \gamma+R_{\infty}^{\prime} \mu^{\perp}(X),
$$

where $\mu=\varrho \gamma+\mu^{\perp}$ is the reverse Lebesgue decomposition given by Lemma 2.3. Then

$$
\mathscr{F}(\gamma \mid \mu)=\mathscr{R}(\mu \mid \gamma) .
$$

Proof It is an immediate consequence of the dual characterization in (2.46) and the equivalence in (2.30).

\section{Optimal Entropy-Transport problems}

The major object of Part I is the entropy-transport functional, where two measures $\mu_{1} \in \mathcal{M}\left(X_{1}\right)$ and $\mu_{2} \in \mathcal{M}\left(X_{2}\right)$ are given, and one has to find a transport plan $\gamma \in \mathcal{M}\left(X_{1} \times X_{2}\right)$ that minimizes the functional.

\subsection{The basic setting}

Let us fix the basic set of data for Entropy-Transport problems. We are given

- two Hausdorff topological spaces $\left(X_{i}, \tau_{i}\right), i=1,2$, which define the Cartesian product $\boldsymbol{X}:=X_{1} \times X_{2}$ and the canonical projections $\pi^{i}: X \rightarrow X_{i}$;

- two entropy functions $F_{i} \in \Gamma\left(\mathbb{R}_{+}\right)$, thus satisfying (2.13);

- a proper, lower semicontinuous cost function c : $\boldsymbol{X} \rightarrow[0, \infty]$;

- a pair of nonnegative Radon measures $\mu_{i} \in \mathcal{M}\left(X_{i}\right)$ with finite mass $m_{i}:=$ $\mu_{i}\left(X_{i}\right)$, satisfying the compatibility condition

$$
J:=\left(m_{1} \mathrm{D}\left(F_{1}\right)\right) \cap\left(m_{2} \mathrm{D}\left(F_{2}\right)\right) \neq \emptyset .
$$


We will often assume that the above basic setting is also coercive: this means that at least one of the following two coercivity conditions holds:

$$
\begin{gathered}
F_{1} \text { and } F_{2} \text { are superlinear, i.e. }\left(F_{i}\right)_{\infty}^{\prime}=+\infty \\
\left(F_{1}\right)_{\infty}^{\prime}+\left(F_{2}\right)_{\infty}^{\prime}+\inf \mathrm{c}>0 \text { and } \mathrm{c} \text { has compact sublevels. }
\end{gathered}
$$

For every transport plan $\boldsymbol{\gamma} \in \mathcal{M}(\boldsymbol{X})$ we define the marginals $\gamma_{i}:=\pi_{\sharp}^{i} \boldsymbol{\gamma}$ and, as in (2.35), we define the relative entropies

$$
\mathscr{F}_{i}\left(\boldsymbol{\gamma} \mid \mu_{i}\right):=\int_{X_{i}} F_{i}\left(\sigma_{i}\right) \mathrm{d} \mu_{i}+\left(F_{i}\right)_{\infty}^{\prime} \gamma_{i}^{\perp}\left(X_{i}\right), \quad \gamma_{i}=\sigma_{i} \mu_{i}+\gamma_{i}^{\perp} .
$$

With this, we introduce the Entropy-Transport functional as

$$
\mathscr{E}\left(\boldsymbol{\gamma} \mid \mu_{1}, \mu_{2}\right):=\sum_{i} \mathscr{F}_{i}\left(\boldsymbol{\gamma} \mid \mu_{i}\right)+\int_{X} \mathrm{c}\left(x_{1}, x_{2}\right) \mathrm{d} \boldsymbol{\gamma}\left(x_{1}, x_{2}\right)
$$

possibly taking the value $+\infty$. Our basic setting is feasible if the functional $\mathscr{E}$ is not identically $+\infty$, i.e. there exists at least one plan $\gamma$ with $\mathscr{E}\left(\gamma \mid \mu_{1}, \mu_{2}\right)<\infty$.

\subsection{The primal formulation of the optimal entropy-transport problem}

In the basic setting described in the previous Sect. 3.1, we want to investigate the following problem.

Problem 3.1 (Entropy-Transport minimization) Given $\mu_{i} \in \mathcal{M}\left(X_{i}\right)$ find $\boldsymbol{\gamma} \in$ $\mathcal{M}(\boldsymbol{X})=\mathcal{M}\left(X_{1} \times X_{2}\right)$ minimizing $\mathscr{E}\left(\boldsymbol{\gamma} \mid \mu_{1}, \mu_{2}\right)$, i.e.

$$
\mathscr{E}\left(\boldsymbol{\gamma} \mid \mu_{1}, \mu_{2}\right)=\mathrm{ET}\left(\mu_{1}, \mu_{2}\right):=\inf _{\boldsymbol{\sigma} \in \mathcal{M}(\boldsymbol{X})} \mathscr{E}\left(\boldsymbol{\sigma} \mid \mu_{1}, \mu_{2}\right)
$$

We denote by $\mathrm{Opt}_{\mathrm{ET}}\left(\mu_{1}, \mu_{2}\right) \subset \mathcal{M}(\boldsymbol{X})$ the collection of all the minimizers of (3.5).

Remark 3.2 (Feasibility conditions) Problem 3.1 is feasible if there exists at least one plan $\boldsymbol{\gamma}$ with $\mathscr{E}\left(\boldsymbol{\gamma} \mid \mu_{1}, \mu_{2}\right)<\infty$. Notice that this is always the case when

$$
F_{i}(0)<\infty, \quad i=1,2,
$$

since among the competitors one can choose the null plan $\eta_{0}$, so that

$$
\mathrm{ET}\left(\mu_{1}, \mu_{2}\right) \leq \mathscr{E}\left(\eta \mid \mu_{1}, \mu_{2}\right)=F_{1}(0) \mu_{1}(X)+F_{2}(0) \mu_{2}(X) .
$$


More generally, thanks to (3.1) a sufficient condition for feasibility in the nondegenerate case $m_{1} m_{2} \neq 0$ is that there exist functions $B_{1}$ and $B_{2}$ with

$$
\mathrm{c}\left(x_{1}, x_{2}\right) \leq B_{1}\left(x_{1}\right)+B_{2}\left(x_{2}\right), \quad B_{i} \in \mathrm{L}^{1}\left(X_{i}, \mu_{i}\right) .
$$

In fact, the plans

$$
\boldsymbol{\gamma}=\frac{\theta}{m_{1} m_{2}} \mu_{1} \otimes \mu_{2} \quad \text { with } \theta \in J \quad \text { given by (3.1) }
$$

are Radon [44, Thm. 17, p. 63], have finite cost $\mathscr{E}\left(\gamma \mid \mu_{1}, \mu_{1}\right)<\infty$ and provide the estimate

$$
\begin{aligned}
\mathrm{ET}\left(\mu_{1}, \mu_{2}\right) \leq \mathscr{E}\left(\boldsymbol{\gamma} \mid \mu_{1}, \mu_{1}\right) \leq & m_{1} F_{1}\left(\theta / m_{1}\right)+m_{2} F_{2}\left(\theta / m_{2}\right) \\
& +\theta \sum_{i} m_{i}{ }^{-1}\left\|B_{i}\right\|_{\mathrm{L}^{1}\left(X_{i}, \mu_{i}\right)}, \text { for every } \theta \in J .
\end{aligned}
$$

Notice that (3.1) is also necessary for feasibility: in fact (2.44) yields

$$
\mathscr{F}_{i}\left(\boldsymbol{\gamma} \mid \mu_{i}\right) \geq m_{i} F_{i}\left(m / m_{i}\right), \text { where } m:=\gamma_{i}\left(X_{i}\right)=\boldsymbol{\gamma}(\boldsymbol{X}) .
$$

Thus, whenever $\mathscr{E}\left(\boldsymbol{\gamma} \mid \mu_{1}, \mu_{2}\right)<\infty$, we have

$$
\mathscr{E}\left(\boldsymbol{\gamma} \mid \mu_{1}, \mu_{2}\right) \geq m \inf \mathrm{C}+m_{1} F_{1}\left(m / m_{1}\right)+m_{2} F_{2}\left(m / m_{2}\right),
$$

and therefore

$$
m=\boldsymbol{\gamma}(\boldsymbol{X}) \in\left(m_{1} \mathrm{D}\left(F_{1}\right)\right) \cap\left(m_{2} \mathrm{D}\left(F_{2}\right)\right)=J .
$$

We will often strengthen (3.1) by assuming that at least one of the domains of the entropies $F_{i}$ has nonempty interior, containing a point of the other domain:

$$
\left(\operatorname{int}\left(m_{1} \mathrm{D}\left(F_{1}\right)\right) \cap m_{2} \mathrm{D}\left(F_{2}\right)\right) \cup\left(m_{1} \mathrm{D}\left(F_{1}\right) \cap \operatorname{int}\left(m_{2} \mathrm{D}\left(F_{2}\right)\right)\right) \neq \emptyset \text {. }
$$

This condition is surely satisfied if $J$ has nonempty interior, i.e. $\max \left(m_{1} s_{1}^{-}\right.$, $\left.m_{2} s_{2}^{-}\right)<\min \left(m_{1} s_{1}^{+}, m_{2} s_{2}^{+}\right)$, where $s_{i}^{-}=\inf \mathrm{D}\left(F_{i}\right), s_{i}^{+}:=\sup \mathrm{D}\left(F_{i}\right)$.

We also observe that whenever $\mu_{i}\left(X_{i}\right)=0$ then the null plan $\gamma:=\eta_{0}$ provides the trivial solution to Problem 3.1. Another trivial case occurs when $F_{i}(0)<\infty$ and $F_{i}$ are nondecreasing in $\mathrm{D}\left(F_{i}\right)$ (in particular when $F_{i}(0)=0$ ). Then it is clear that the null plan is a minimizer and $\mathrm{ET}\left(\mu_{1}, \mu_{2}\right)=F_{1}(0) m_{1}+$ $F_{2}(0) m_{2}$. 


\subsection{Examples}

Let us consider a few particular cases:

E. 1 Costless transport: Consider the case $c \equiv 0$. Since $F_{i}$ are convex, in this case the minimum is attained when the marginals $\gamma_{i}$ have constant densities. Setting $\sigma_{i} \equiv \theta / m_{i}$ in order to have $m_{1} \sigma_{1}=m_{2} \sigma_{2}$, we thus have

$\mathrm{ET}\left(\mu_{1}, \mu_{2}\right)=H_{0}\left(m_{1}, m_{2}\right):=\min \left\{m_{1} F_{1}\left(\theta / m_{1}\right)+m_{2} F_{2}\left(\theta / m_{2}\right): \theta \geq 0\right\}$.

E.2 Entropy-potential problems: If $\mu_{2} \equiv \eta_{0}$ is the null measure and, just to fix ideas, $X_{i}$ are Polish spaces with $X_{2}$ compact and $\mathrm{c}$ is real valued, then setting $V\left(x_{1}\right):=\min _{x_{2} \in X_{2}} \mathrm{C}\left(x_{1}, x_{2}\right)$ we get

$$
\mathrm{ET}\left(\mu, \eta_{0}\right)=\inf _{\gamma \in \mathcal{M}\left(X_{1}\right)} \mathscr{F}_{1}(\gamma \mid \mu)+\int_{X_{1}} V \mathrm{~d} \gamma+\left(F_{2}\right)_{\infty}^{\prime} \gamma\left(X_{1}\right) .
$$

In fact for every $\boldsymbol{\gamma} \in \mathcal{M}(\boldsymbol{X})$ we have $\mathscr{F}_{2}\left(\gamma_{2} \mid \eta_{0}\right)=\left(F_{2}\right)_{\infty}^{\prime} \gamma_{2}\left(X_{2}\right)=$ $\left(F_{2}\right)_{\infty}^{\prime} \gamma_{1}\left(X_{1}\right)$; moreover by applying the von Neumann measurable selection Theorem [44, Thm. 13, p. 127] it is not difficult to check that

$$
\min \left\{\int_{\boldsymbol{X}} \mathrm{cd} \boldsymbol{\gamma}: \boldsymbol{\gamma} \in \mathcal{M}(\boldsymbol{X}), \pi_{\sharp}^{1} \boldsymbol{\gamma}=\gamma\right\}=\int_{X_{1}} V\left(x_{1}\right) \mathrm{d} \gamma\left(x_{1}\right) .
$$

E.3 Pure transport problems: We choose $F_{i}(r)=\mathrm{I}_{1}(r)= \begin{cases}0 & \text { if } r=1 \\ +\infty & \text { otherwise. }\end{cases}$ In this case any feasible plan $\gamma$ should have $\mu_{1}$ and $\mu_{2}$ as marginals and the functional just reduces to the pure transport part

$$
\mathrm{T}\left(\mu_{1}, \mu_{2}\right)=\min \left\{\int_{X_{1} \times X_{2}} \mathrm{~cd} \boldsymbol{\gamma}: \pi_{\sharp}^{i} \boldsymbol{\gamma}=\mu_{i}\right\} .
$$

As a necessary condition for feasibility we get $\mu_{1}\left(X_{1}\right)=\mu_{2}\left(X_{2}\right)$.

A situation equivalent to the optimal transport case occurs when (3.12) does not hold. In this case, the set $J$ defined by (3.1) contains only one point $\theta$ which separates $m_{1} \mathrm{D}\left(F_{1}\right)$ and $m_{2} \mathrm{D}\left(F_{2}\right)$ :

$$
\theta=m_{1} s_{1}^{+}=m_{2} s_{2}^{-} \quad \text { or } \quad \theta=m_{1} s_{1}^{-}=m_{2} s_{2}^{+} .
$$

It is not difficult to check that in this case

$$
\mathrm{ET}\left(\mu_{1}, \mu_{2}\right)=m_{1} F_{1}\left(\theta / m_{1}\right)+m_{2} F_{2}\left(\theta / m_{2}\right)+\mathrm{T}\left(\mu_{1}, \mu_{2}\right) .
$$


E.4 Optimal transport with density constraints: We realize density constraints by introducing characteristic functions of intervals $\left[a_{i}, b_{i}\right]$, viz. $F_{i}(r):=\mathrm{I}_{\left[a_{i}, b_{i}\right]}(r), a_{i} \leq 1 \leq b_{i}$. E.g. when $a_{i}=1, b_{i}=+\infty$ we have

$$
\mathrm{ET}\left(\mu_{1}, \mu_{2}\right)=\min \left\{\int_{X_{1} \times X_{2}} \mathrm{~cd} \boldsymbol{\gamma}: \pi_{\sharp}^{i} \boldsymbol{\gamma} \geq \mu_{i}\right\} .
$$

For $\left[a_{1}, b_{1}\right]=[0,1]$ and $\left[a_{2}, b_{2}\right]=[1, \infty]$ we get

$$
\mathrm{ET}\left(\mu_{1}, \mu_{2}\right)=\min \left\{\int_{X_{1} \times X_{2}} \mathrm{~cd} \boldsymbol{\gamma}: \quad \pi_{\sharp}^{1} \gamma \leq \mu_{1}, \pi_{\sharp}^{2} \boldsymbol{\gamma} \geq \mu_{2}\right\},
$$

whose feasibility requires $\mu_{2}\left(X_{2}\right) \geq \mu_{1}\left(X_{1}\right)$.

E.5 Pure entropy problems: These problems arise if $X_{1}=X_{2}=X$ and transport is forbidden, i.e. $\left(F_{i}\right)_{\infty}^{\prime}=+\infty, \mathrm{c}\left(x_{1}, x_{2}\right)= \begin{cases}0 & \text { if } x_{1}=x_{2} \\ +\infty & \text { otherwise. }\end{cases}$ In this case the marginals of $\gamma$ coincide: we denote them by $\gamma$. We can write the density of $\gamma$ w.r.t. any measure $\mu$ such that $\mu_{i} \ll \mu$ (say, e.g., $\left.\mu=\mu_{1}+\mu_{2}\right)$ as $\gamma=\vartheta \mu$ and then $\mu_{i}=\vartheta_{i} \mu$. Since $\gamma \ll \mu_{i}$ we have $\vartheta(x)=0$ for $\mu$-a.e. $x$ where $\vartheta_{1}(x) \vartheta_{2}(x)=0$. Thus $\sigma_{i}=\vartheta / \vartheta_{i}$ is well defined and we have

$$
\mathscr{E}\left(\gamma \mid \mu_{1}, \mu_{2}\right)=\int_{X}\left(\vartheta_{1} F_{1}\left(\vartheta / \vartheta_{1}\right)+\vartheta_{2} F_{2}\left(\vartheta / \vartheta_{2}\right)\right) \mathrm{d} \mu,
$$

with the convention that $\vartheta_{i} F_{i}\left(\vartheta / \vartheta_{i}\right)=0$ if $\vartheta=\vartheta_{i}=0$. Since we expressed everything in terms of $\mu$, by recalling the definition of the function $H_{0}$ given in (3.13) we get

$$
\operatorname{ET}\left(\mu_{1}, \mu_{2}\right)=\int_{X} H_{0}\left(\frac{\mathrm{d} \mu_{1}}{\mathrm{~d} \mu}, \frac{\mathrm{d} \mu_{2}}{\mathrm{~d} \mu}\right) \mathrm{d} \mu, \quad \text { whenever } \mu_{i} \ll \mu .
$$

In the Hellinger case $F_{i}(s)=U_{1}(s)=s \log s-s+1$ a simple calculation yields

$$
H_{0}\left(\theta_{1}, \theta_{2}\right)=\theta_{1}+\theta_{2}-2 \sqrt{\theta_{1} \theta_{2}}=\left(\sqrt{\theta_{1}}-\sqrt{\theta_{2}}\right)^{2} .
$$

In the Jensen-Shannon case, where $F_{i}(s)=U_{0}(s)=s-1-\log s$, we obtain

$$
H_{0}\left(\theta_{1} ; \theta_{2}\right)=\theta_{1} \log \left(\frac{2 \theta_{1}}{\theta_{1}+\theta_{2}}\right)+\theta_{2} \log \left(\frac{2 \theta_{2}}{\theta_{1}+\theta_{2}}\right) .
$$


Two other interesting examples are provided by the quadratic case $F_{i}(s)=$ $\frac{1}{2}(s-1)^{2}$ and by the nonsmooth "piecewise affine" case $F_{i}(s)=|s-1|$, for which we obtain

$$
\begin{aligned}
& H_{0}\left(\theta_{1}, \theta_{2}\right)=\frac{1}{2\left(\theta_{1}+\theta_{2}\right)}\left(\theta_{1}-\theta_{2}\right)^{2}, \quad \text { and } \\
& H_{0}\left(\theta_{1}, \theta_{2}\right)=\left|\theta_{1}-\theta_{2}\right|, \quad \text { respectively. }
\end{aligned}
$$

E.6 Regular Entropy-Transport problems: These problems correspond to the choice of a pair of differentiable entropies $F_{i}$ with $\mathrm{D}\left(F_{i}\right) \supset(0, \infty)$, as in the case of the power-like entropies $U_{p}$ defined in (2.26). When they vanish (and thus have a minimum) at $s=1$, the Entropic Optimal Transportation can be considered as a smooth relaxation of the Optimal Transport case E.3.

E.7 Squared Hellinger-Kantorovich distances: For a metric space $(X, \mathrm{~d})$, set $X_{1}=X_{2}=X$ and let $\tau$ be induced by d. Further, set $F_{1}(s)=$ $F_{2}(s):=U_{1}(s)=s \log s-s+1$ and

$$
\begin{aligned}
& \mathrm{c}\left(x_{1}, x_{2}\right):=-\log \left(\cos ^{2}\left(\mathrm{~d}\left(x_{1}, x_{2}\right) \wedge \pi / 2\right)\right) \text { or simply } \\
& \mathrm{c}\left(x_{1}, x_{2}\right):=\mathrm{d}^{2}\left(x_{1}, x_{2}\right)
\end{aligned}
$$

This case will be thoroughly studied in the second part of the present paper, see Sect. 6.

E.8 Marginal Entropy-Transport problems: In this case one of the two marginals of $\gamma$ is fixed, say $\gamma_{1}$, by choosing $F_{1}(r):=\mathrm{I}_{1}(r)$. Thus the functional minimizes the sum of the transport cost and the relative entropy of the second marginal $\mathscr{F}_{2}\left(\gamma_{2} \mid \mu_{2}\right)$ with respect to a reference measure $\mu_{2}$, namely

$$
\mathrm{ET}\left(\mu_{1}, \mu_{2}\right)=\min _{\gamma_{2} \in \mathcal{M}\left(X_{2}\right)}\left\{\mathrm{T}\left(\mu_{1}, \gamma_{2}\right)+\mathscr{F}_{2}\left(\gamma_{2} \mid \mu_{2}\right)\right\}
$$

where $T$ has been defined by (3.15). This is the typical situation one has to solve at each iteration step of the Minimizing Movement scheme [2], when $\mathrm{T}$ is a (power of a) transport distance induced by $\mathrm{C}$, as in the JordanKinderlehrer-Otto approach [24]. 
E.9 The Piccoli-Rossi "generalized Wasserstein distance" [38,39]: For a metric space $(X, \mathrm{~d})$, set $X_{1}=X_{2}=X$, let $\tau$ be induced by d, and consider $F_{1}(s)=F_{2}(s):=V(s)=|s-1|$ with $\mathrm{c}\left(x_{1}, x_{2}\right):=$ $\mathrm{d}\left(x_{1}, x_{2}\right)$. This example can be considered as the natural extension of the $L^{1}$-Kantorovich-Wasserstein distance (corresponding to (3.15) with the distance cost) to measures with different masses, due to its dual representation in terms of the flat metric, see (7.47).

E.10 The discrete case. Let $\mu_{1}=\sum_{i=1}^{m} \alpha_{i} \delta_{x_{i}}, \mu_{2}=\sum_{j=1}^{N} \beta_{j} \delta_{y_{j}}$ with $\alpha_{i}, \beta_{j}>0$, and let $\mathrm{c}_{i, j}:=\mathrm{C}\left(x_{i}, y_{j}\right)$. In the case of superlinear entropy functions $F_{i}$, the Entropy-Transport problem for this discrete model consists in finding coefficients $\gamma_{i, j} \geq 0$ which minimize

$$
\mathscr{E}\left(\gamma_{i, j} \mid \alpha_{i}, \beta_{j}\right):=\sum_{i} \alpha_{i} F_{1}\left(\frac{\sum_{j} \gamma_{i, j}}{\alpha_{i}}\right)+\sum_{j} \beta_{j} F_{2}\left(\frac{\sum_{i} \gamma_{i, j}}{\beta_{j}}\right)+\sum_{i, j} \mathrm{c}_{i, j} \gamma_{i, j}
$$

\subsection{Existence of solutions to the primal problem}

The next result provides a first general existence result for Problem 3.1 in the basic coercive setting of Sect. 3.1.

Theorem 3.3 (Existence of minimizers) Let us assume that Problem 3.1 is feasible (see Remark 3.2) and coercive, namely at least one of the following conditions hold:

(i) the entropy functions $F_{1}$ and $F_{2}$ are superlinear, i.e. $\left(F_{1}\right)_{\infty}^{\prime}=\left(F_{2}\right)_{\infty}^{\prime}=$ $+\infty$;

(ii) c has compact sublevels in $\boldsymbol{X}$ and $\left(F_{1}\right)_{\infty}^{\prime}+\left(F_{2}\right)_{\infty}^{\prime}+\inf \mathrm{c}>0$.

Then Problem 3.1 admits at least one optimal solution. In this case $\mathrm{Opt}_{\mathrm{ET}}\left(\mu_{1}, \mu_{2}\right)$ is a compact convex set of $\mathcal{M}(\boldsymbol{X})$.

Proof We can apply the Direct Method of Calculus of Variations: since the map $\boldsymbol{\gamma} \mapsto \mathscr{E}\left(\boldsymbol{\gamma} \mid \mu_{1}, \mu_{2}\right)$ is lower semicontinuous in $\mathcal{M}\left(X_{1} \times X_{2}\right)$ by Theorem 2.7, it is sufficient to show that its sublevels are relatively compact, thus bounded and equally tight by the Prokhorov Theorem 2.2. In both cases boundedness follows by the coercivity assumptions and the estimate (3.10). In particular, by formula (2.15) defining $\left(F_{i}\right)_{\infty}^{\prime}$, we can find $\bar{s} \geq 0$ such that $\frac{m_{i}}{m} F_{i}\left(\frac{m}{m_{i}}\right) \geq \frac{1}{2}\left(F_{i}\right)_{\infty}^{\prime}$ whenever $m \geq \bar{s} m_{i}$; if $a:=\inf c+\sum_{i}\left(F_{i}\right)_{\infty}^{\prime}>0$ the estimate (3.10) yields

$$
\boldsymbol{\gamma}(\boldsymbol{X}) \leq \frac{2}{a} \mathscr{E}\left(\boldsymbol{\gamma} \mid \mu_{1}, \mu_{2}\right) \text { for every } \boldsymbol{\gamma} \in \mathcal{M}(\boldsymbol{X}) \text { with } \boldsymbol{\gamma}(\boldsymbol{X})
$$




$$
\geq \bar{s} \max \left(\mu_{1}\left(X_{1}\right), \mu_{2}\left(X_{2}\right)\right) \text {. }
$$

In case (ii) equal tightness is a consequence of the Markov inequality and the nonnegativity of $F_{i}$ : by considering the compact sublevels $K_{\lambda}:=\left\{\left(x_{1}, x_{2}\right) \in\right.$ $\left.X_{1} \times X_{2}: \mathrm{c}\left(x_{1}, x_{2}\right) \leq \lambda\right\}$, we have

$$
\boldsymbol{\gamma}\left(\boldsymbol{X} \backslash K_{\lambda}\right) \leq \lambda^{-1} \int \mathrm{cd} \boldsymbol{\gamma} \leq \lambda^{-1} \mathscr{E}\left(\gamma \mid \mu_{1}, \mu_{2}\right) \text { for every } \lambda>0 .
$$

In the case $(i)$, since $c \geq 0$ Proposition 2.10 shows that both the marginals of plans in a sublevel of the energy are equally tight and we thus conclude by [2, Lemma 5.2.2].

Remark 3.4 The assumptions (i) and (ii) in the previous theorem are almost optimal, and it is not hard to find examples violating them such that the statement of Theorem 3.3 does not hold. In the case when $0<\left(F_{1}\right)_{\infty}^{\prime}+\left(F_{2}\right)_{\infty}^{\prime}<\infty$ but $\mathrm{C}$ does not have compact sublevels, one can just take $F_{i}(s):=U_{0}(s)=$ $s-\log s-1, X_{i}:=\mathbb{R}, \mathrm{C}\left(x_{1}, x_{2}\right):=3 \mathrm{e}^{-x_{1}^{2}-x_{2}^{2}}, \mu_{i}=\delta_{0}$.

Any competitor is of the form $\gamma:=\alpha \delta_{0} \otimes \delta_{0}+v_{1} \otimes \delta_{0}+\delta_{0} \otimes v_{2}$ with $v_{i} \in \mathcal{M}(\mathbb{R})$ and $v_{i}(\{0\})=0$. Setting $n_{i}:=v_{i}(\mathbb{R})$ we find

$$
\begin{aligned}
\mathscr{E}\left(\gamma \mid \mu_{1}, \mu_{2}\right)= & F\left(\alpha+n_{1}\right)+F\left(\alpha+n_{2}\right) \\
& +3\left(\alpha+\int \mathrm{e}^{-x^{2}} \mathrm{~d}\left(v_{1}+v_{2}\right)\right)+n_{1}+n_{2} .
\end{aligned}
$$

Since $\min _{s} F(s)+s=\log 2$ is attained at $s=1 / 2$, we immediately see that

$$
\mathscr{E}\left(\gamma \mid \mu_{1}, \mu_{2}\right) \geq 2 \log 2+\alpha+3 \int \mathrm{e}^{-x^{2}} \mathrm{~d}\left(v_{1}+v_{2}\right) \geq 2 \log 2 .
$$

Moreover, $2 \log 2$ is the infimum, which is reached by choosing $\alpha=0$ and $v_{1}=v_{2}=\frac{1}{2} \delta_{x}$, and letting $x \rightarrow+\infty$. On the other hand, since $n_{1}+n_{2}+\alpha>0$, the infimum can never be attained.

In the case when $\mathrm{c}$ has compact sublevels but $\left(F_{1}\right)_{\infty}^{\prime}=\left(F_{2}\right)_{\infty}^{\prime}=\min \mathrm{c}=0$, it is sufficient to take $F_{i}(s):=s^{-1}, X_{i}=[-1,1], \mathrm{C}\left(x_{1}, x_{2}\right)=x_{1}^{2}+x_{2}^{2}$, and $\mu_{i}=\delta_{0}$. Taking $\gamma_{n}:=n \delta_{0} \otimes \delta_{0}$ one easily checks that inf $\mathscr{E}\left(\boldsymbol{\gamma} \mid \mu_{1}, \mu_{2}\right)=0$ but $\mathscr{E}\left(\boldsymbol{\gamma} \mid \mu_{1}, \mu_{2}\right)>0$ for every $\boldsymbol{\gamma} \in \mathcal{M}\left(\mathbb{R}^{2}\right)$.

Let us briefly discuss the question of uniqueness. The first result only addresses the marginals $\gamma_{i}=\pi_{\sharp}^{i} \gamma$.

Lemma 3.5 (Uniqueness of the marginals in the superlinear, strictly convex case) Let us suppose that $F_{i}$ are strictly convex functions. Then the $\mu_{i}$ absolutely continuous part $\sigma_{i} \mu_{i}$ of the marginals $\gamma_{i}=\pi_{\sharp}^{i} \gamma$ of any optimal 
plan are uniquely determined. In particular, if $F_{i}$ are also superlinear, then the marginals $\gamma_{i}$ are uniquely determined, i.e. if $\boldsymbol{\gamma}^{\prime}, \boldsymbol{\gamma}^{\prime \prime} \in \mathrm{Opt}_{\mathrm{ET}}\left(\mu_{1}, \mu_{2}\right)$ then $\pi_{\sharp}^{i} \boldsymbol{\gamma}^{\prime}=\pi_{\sharp}^{i} \boldsymbol{\gamma}^{\prime \prime}, i=1,2$.

Proof It is sufficient to take $\boldsymbol{\gamma}=\frac{1}{2} \boldsymbol{\gamma}^{\prime}+\frac{1}{2} \boldsymbol{\gamma}^{\prime \prime}$ which is still optimal in $\operatorname{Opt}_{\mathrm{ET}}\left(\mu_{1}, \mu_{2}\right)$ since $\mathscr{E}$ is a convex functional w.r.t. $\boldsymbol{\gamma}$. We have $\pi_{\sharp}^{i} \boldsymbol{\gamma}=\gamma_{i}=$ $\frac{1}{2} \gamma_{i}^{\prime}+\frac{1}{2} \gamma_{i}^{\prime \prime}=\frac{1}{2}\left(\sigma_{i}^{\prime}+\sigma_{i}^{\prime \prime}\right) \mu+\frac{1}{2}\left(\gamma_{i}^{\prime}\right)^{\perp}+\frac{1}{2}\left(\gamma_{i}^{\prime \prime}\right)^{\perp}$ and we observe that the minimality of $\gamma$ and the convexity of each addendum $F_{i}$ in the functional yield

$$
\mathscr{F}_{i}\left(\gamma_{i} \mid \mu_{i}\right)=\frac{1}{2} \mathscr{F}_{i}\left(\gamma_{i}^{\prime} \mid \mu_{i}\right)+\frac{1}{2} \mathscr{F}_{i}\left(\gamma_{i}^{\prime \prime} \mid \mu_{i}\right) \text { for } i=1,2
$$

Since $\gamma_{i}^{\perp}\left(X_{i}\right)=\frac{1}{2}\left(\gamma_{i}^{\prime}\right)^{\perp}\left(X_{i}\right)+\frac{1}{2}\left(\gamma_{i}^{\prime \prime}\right)^{\perp}\left(X_{i}\right)$ we obtain

$$
\int_{X}\left(F_{i}\left(\sigma_{i}\right)-\frac{1}{2} F_{i}\left(\sigma_{i}^{\prime}\right)-\frac{1}{2} F_{i}\left(\sigma_{i}^{\prime \prime}\right)\right) \mathrm{d} \mu_{i}=0 \text { for } i=1,2 .
$$

Since $F_{i}$ is strictly convex, the above identity implies $\sigma_{i}=\sigma_{i}^{\prime}=\sigma_{i}^{\prime \prime} \mu_{i}$-a.e. in $X$. When $F_{i}$ are superlinear then $\gamma_{i}=\sigma_{i}$ thanks to (2.36).

The next corollary reduces the uniqueness of optimal couplings in $\mathrm{Opt}_{\mathrm{ET}}\left(\mu_{1}, \mu_{2}\right)$ to corresponding results for the Kantorovich problem associated to the cost $\mathbf{C}$.

Corollary 3.6 Let us suppose that $F_{i}$ are superlinear strictly convex functions and that for every pair of probability measures $v_{i} \in \mathcal{P}\left(X_{i}\right)$ with $v_{i} \ll \mu_{i}$ the optimal transport problem associated to the cost $\mathrm{C}$ (see Example E.3 of Sect. 3.3) admits a unique solution. Then $\mathrm{Opt}_{\mathrm{ET}}\left(\mu_{1}, \mu_{2}\right)$ contains at most one plan.

Proof We can assume $m_{i}=\mu_{i}\left(X_{i}\right)>0$ for $i=1,2$. It is clear that any $\gamma \in \mathrm{Opt}_{\mathrm{ET}}\left(\mu_{1}, \mu_{2}\right)$ is a solution of the optimal transport problem for the cost $\mathrm{C}$ and given marginals $\gamma_{i}$. Since $\gamma_{i} \ll \mu_{i}$ by (2.36) and $\gamma_{1}$ and $\gamma_{2}$ are unique by Lemma 3.5, we conclude.

Example 3.7 (Uniqueness in Euclidean spaces) If $F_{i}$ are superlinear strictly convex functions, $\mathrm{C}(x, y)=h(x-y)$ for a strictly convex function $h: \mathbb{R}^{d} \rightarrow$ $[0, \infty)$ and $\mu_{1} \ll \mathscr{L}^{d}$, then Problem 3.1 admits at most one solution. It is sufficient to apply the previous corollary in conjunction with [2, Theorem $6.2 .4]$

Example 3.8 (Nonuniqueness of optimal couplings) Consider the logarithmic density functionals $F_{i}(s)=U_{1}(s)=s \log s-s+1$, the Euclidean space 
$X_{1}=X_{2}=\mathbb{R}^{2}$ and any cost $\mathrm{c}$ of the form $\mathrm{c}\left(x_{1}, x_{2}\right)=h\left(\left|x_{1}-x_{2}\right|\right)$. For the measures $\mu_{1}=\delta_{(-1,0)}+\delta_{(1,0)}$, and $\mu_{2}$ with support in $\{0\} \times \mathbb{R}$ and containing at least two points, there are an infinite number of optimal plans. In fact, we shall see that the first marginal $\gamma_{1}$ of any optimal plan $\gamma$ will have full support in $A:=\{(-1,0),(1,0)\}$, i.e. it will of the form $a \delta_{(-1,0)}+b \delta_{(1,0)}$ with strictly positive $a, b$, and the support of the second marginal $\gamma_{2}$ will be concentrated in $B:=\{0\} \times \mathbb{R}$ and will contain at least two points. Any plan $\sigma$ with marginals $\gamma_{1}, \gamma_{2}$ will then be optimal, since it will be supported in $A \times B$ where the cost C just depends on the second variable, since $|( \pm 1,0)-(0, y)|=\sqrt{1+y^{2}}$ for every $y \in \mathbb{R}$. Therefore the cost contribution of $\sigma$ to the total energy is

$$
\int_{\mathbb{R}} h\left(\sqrt{1+y^{2}}\right) \mathrm{d} \gamma_{2}(y),
$$

and we can choose $\sigma$ of the form [2, Sect.5.3]

$$
\boldsymbol{\sigma}=\int_{\mathbb{R}}\left(\alpha(y) \delta_{(-1,0)}+\beta(y) \delta_{(1,0)}\right) \mathrm{d} \gamma_{2}(y),
$$

with arbitrary nonnegative densities $\alpha, \beta$ satisfying $\alpha+\beta=1$ and $\int \alpha \mathrm{d} \gamma_{2}(y)=a, \int \beta \mathrm{d} \gamma_{2}(y)=b$ will be admissible.

We conclude this section by proving a simple lower semicontinuity property for the energy-transport functional ET. Note that in metrizable spaces any weakly convergent sequence of Radon measures is equally tight.

Lemma 3.9 Let $\mathbb{L}$ be a directed set, $\left(F_{i}^{\lambda}\right)_{\lambda \in \mathbb{L}}$ and $\left(\mathrm{c}^{\lambda}\right)_{\lambda \in \mathbb{L}}$ be monotone nets of superlinear entropies and costs pointwise converging to $F_{i}$ and $\mathrm{c}$ respectively, and let $\left(\mu_{i}^{\lambda}\right)_{\lambda \in \mathbb{L}}$ be equally tight nets of measures narrowly converging to $\mu_{i}$ in $\mathcal{M}\left(X_{i}\right)$. Denoting by $\mathrm{ET}^{\lambda}$ (resp. $\mathrm{ET}$ ) the corresponding Entropy-Transport functionals induced by $F_{i}^{\lambda}$ and $\mathrm{c}^{\lambda}$ (resp. $F_{i}$ and $\mathrm{c}$ ) we have

$$
\liminf _{\lambda \in \mathbb{L}} \mathbb{E}^{\lambda}\left(\mu_{1}^{\lambda}, \mu_{2}^{\lambda}\right) \geq \mathbb{E T}\left(\mu_{1}, \mu_{2}\right) .
$$

Proof Let $\gamma^{\lambda} \in \mathrm{Opt}_{\mathrm{ET}}\left(\mu_{1}^{\lambda}, \mu_{2}^{\lambda}\right) \subset \mathcal{M}(\boldsymbol{X})$ be a corresponding net of optimal plans. The statement follows if, assuming that $\mathscr{E}\left(\boldsymbol{\gamma}^{\lambda} \mid \mu_{1}^{\lambda}, \mu_{2}^{\lambda}\right)=\mathrm{ET}\left(\mu_{1}^{\lambda}, \mu_{2}^{\lambda}\right) \leq$ $C<\infty$, we can prove that $\mathrm{ET}\left(\mu_{1}, \mu_{2}\right) \leq C$. By applying Proposition 2.10, we obtain that the sequences of marginals $\pi_{\sharp}^{i} \gamma^{\lambda}$ are equally tight in $\mathcal{M}\left(X_{i}\right)$, so that the net $\gamma^{\lambda}$ is also equally tight by [2, Lemma 5.2.2]. By extracting a suitable subnet (not relabeled) narrowly converging to $\boldsymbol{\gamma}$ in $\mathcal{N}(\boldsymbol{X})$, we can still apply Proposition 2.10 and Corollary 2.9 to obtain

$$
\liminf _{\lambda \in \mathbb{L}} \sum_{i} \mathscr{F}_{i}^{\lambda}\left(\boldsymbol{\gamma}^{\lambda} \mid \mu_{i}^{\lambda}\right) \geq \sum_{i} \mathscr{F}_{i}\left(\boldsymbol{\gamma} \mid \mu_{i}\right)
$$


A standard monotonicity argument and the lower semicontinuity of the cost functions $\mathrm{C}^{\lambda}$ show that for every $\alpha \in \mathbb{L}$

$$
\liminf _{\lambda \in \mathbb{L}} \int c^{\lambda} d \boldsymbol{\gamma}^{\lambda} \geq \liminf _{\lambda \in \mathbb{L}} \int c^{\alpha} d \boldsymbol{\gamma}^{\lambda} \geq \int c^{\alpha} d \boldsymbol{\gamma} .
$$

Passing now to the limit with respect to $\alpha \in \mathbb{L}$ and recalling (2.2) we conclude.

As a simple application we prove the extremality of the class of Optimal Transport problems (see Example E.3 in Sect. 3.3) in the set of entropytransport problems.

Corollary 3.10 Let $F_{1}, F_{2} \in \Gamma\left(\mathbb{R}_{+}\right)$satisfy $F_{i}(r)>F_{i}(1)=0$ for every $r \in[0, \infty), r \neq 1$ and let $\mathrm{ET}^{n}$ be the Optimal Entropy Transport value (3.5) associated to $\left(n F_{1}, n F_{2}\right)$. Then for every pair of equally tight sequences $\left(\mu_{1, n}, \mu_{2, n}\right) \subset \mathcal{M}\left(X_{1}\right) \times \mathcal{M}\left(X_{2}\right), n \in \mathbb{N}$, narrowly converging to $\left(\mu_{1}, \mu_{2}\right)$ we have

$$
\lim _{n \uparrow \infty} \operatorname{ET}^{n}\left(\mu_{1, n}, \mu_{2, n}\right)=\mathrm{T}\left(\mu_{1}, \mu_{2}\right) .
$$

\subsection{The reverse formulation of the primal problem}

Let us recall the definition (2.28) of the reverse entropy functions $R_{i}$ associated to $F_{i}$ by the formula

$$
R_{i}(r):= \begin{cases}r F_{i}(1 / r) & \text { if } r>0, \\ \left(F_{i}\right)_{\infty}^{\prime} & \text { if } r=0,\end{cases}
$$

and let $\mathscr{R}_{i}$ be the corresponding integral functionals as in (2.57).

Keeping the notation of Lemma 2.3

$$
\gamma_{i}:=\pi_{\sharp}^{i} \gamma \in \mathcal{M}\left(X_{i}\right), \quad \mu_{i}=\varrho_{i} \gamma_{i}+\mu_{i}^{\perp},
$$

we can thus define

$$
\begin{aligned}
& \mathscr{R}\left(\mu_{1}, \mu_{2} \mid \boldsymbol{\gamma}\right):=\sum_{i} \mathscr{R}_{i}\left(\mu_{i} \mid \gamma_{i}\right)+\int_{X} \mathrm{~cd} \boldsymbol{\gamma} \\
& \quad=\int_{X}\left(R_{1}\left(\varrho_{1}\left(x_{1}\right)\right)+R_{2}\left(\varrho_{2}\left(x_{2}\right)\right)+\mathrm{c}\left(x_{1}, x_{2}\right)\right) \mathrm{d} \boldsymbol{\gamma}+\sum_{i} F_{i}(0) \mu_{i}^{\perp}\left(X_{i}\right) .
\end{aligned}
$$

By Lemma 2.11 we easily get the reverse formulation of the optimal EntropyTransport Problem 3.1. 
Theorem 3.11 For every $\boldsymbol{\gamma} \in \mathcal{M}(\boldsymbol{X})$ and $\mu_{i} \in \mathcal{M}\left(X_{i}\right)$

$$
\mathscr{E}\left(\boldsymbol{\gamma} \mid \mu_{1}, \mu_{2}\right)=\mathscr{R}\left(\mu_{1}, \mu_{2} \mid \boldsymbol{\gamma}\right)
$$

In particular

$$
\mathrm{ET}\left(\mu_{1}, \mu_{2}\right)=\inf _{\boldsymbol{\gamma} \in \mathcal{M}(\boldsymbol{X})} \mathscr{R}\left(\mu_{1}, \mu_{2} \mid \boldsymbol{\gamma}\right),
$$

and $\boldsymbol{\gamma} \in \mathrm{Opt}_{\mathrm{ET}}\left(\mu_{1}, \mu_{2}\right)$ if and only if it minimizes $\mathscr{R}\left(\mu_{1}, \mu_{2} \mid \cdot\right)$ in $\mathcal{M}(\boldsymbol{X})$.

The functional $\mathscr{R}\left(\mu_{1}, \mu_{2} \mid, \cdot\right)$ is still a convex functional, and it will be useful in Sect. 5.

\section{The dual problem}

In this section we want to compute and study the dual problem and the corresponding optimality conditions for the Entropy-Transport Problem 3.1 in the basic coercive setting of Sect. 3.1. The derivation of the dual problem will be carried out in Sect. 4.1 by writing a saddle-point formulation of the primal problem 3.1 based on the duality Theorem 2.7 for the entropy functionals $\mathscr{F}_{i}$. The subsequent sections will then perform a systematic study of the duality and of the related optimality conditions.

\subsection{The "inf-sup" derivation of the dual problem in the basic coercive setting}

In order to write the first formulation of the dual problem we will use the reverse entropy functions $R_{i}$ defined as in (2.28) or Sect. 3.5 and their conjugates $R_{i}^{*}: \mathbb{R} \rightarrow(-\infty,+\infty]$, which can be expressed by

$$
R_{i}^{*}(\psi):=\sup _{s>0}\left(s \psi-s F_{i}(1 / s)\right)=\sup _{r>0}\left(\psi-F_{i}(r)\right) / r .
$$

The equivalences (2.31) yield, for all $(\phi, \psi) \in \mathbb{R}^{2}$, the equivalence

$$
(\phi, \psi) \in \mathfrak{F}_{i} \quad \Leftrightarrow \quad \phi \leq-R_{i}^{*}(\psi) .
$$

As a first step we use the dual formulation of the entropy functionals given by Theorem 2.7 (cf. (2.47)) and find

$$
\begin{gathered}
\mathscr{E}\left(\boldsymbol{\gamma} \mid \mu_{1}, \mu_{2}\right)=\int \mathrm{cd} \boldsymbol{\gamma}+\sup \left\{\sum_{i}\left(\int_{X_{i}} \psi_{i} \mathrm{~d} \mu_{i}-\sum_{i} \int_{X_{i}} R_{i}^{*}\left(\psi_{i}\right) \mathrm{d} \gamma_{i}\right):\right. \\
\left.\psi_{i} \in \operatorname{LSC}_{S}\left(X_{i}, \stackrel{\circ}{\mathrm{D}}\left(R_{i}^{*}\right)\right)\right\} .
\end{gathered}
$$


It is now natural to introduce the saddle function $\mathscr{L}(\boldsymbol{\gamma}, \boldsymbol{\psi})$ depending on $\boldsymbol{\gamma} \in \mathcal{M}(\boldsymbol{X})$ and $\boldsymbol{\psi}=\left(\psi_{1}, \psi_{2}\right)$ with $\psi_{i} \in \mathrm{LSC}_{s}\left(X_{i}, \mathrm{D}\left(R_{i}^{*}\right)\right)$ (omitting the dependence on the fixed measures $\left.\mu_{i} \in \mathcal{M}\left(X_{i}\right)\right)$ via

$$
\mathscr{L}(\boldsymbol{\gamma}, \boldsymbol{\psi}):=\int_{X}\left(\mathrm{c}\left(x_{1}, x_{2}\right)-R_{1}^{*}\left(\psi_{1}\left(x_{1}\right)\right)-R_{2}^{*}\left(\psi_{2}\left(x_{2}\right)\right)\right) \mathrm{d} \boldsymbol{\gamma}+\sum_{i} \int_{X_{i}} \psi_{i} \mathrm{~d} \mu_{i}
$$

Notice that $R_{i}^{*}\left(\psi_{i}\right)$ are bounded, so $\mathscr{L}$ cannot take the value $-\infty$; in order to guarantee that $\mathscr{L}<+\infty$, we consider the convex set

$$
\mathrm{M}:=\left\{\boldsymbol{\gamma} \in \mathcal{M}(\boldsymbol{X}): \int \mathrm{cd} \boldsymbol{\gamma}<\infty\right\} .
$$

We thus have

$$
\mathscr{E}\left(\boldsymbol{\gamma} \mid \mu_{1}, \mu_{2}\right)=\sup _{\psi_{i} \in \operatorname{LSC}_{s}\left(X_{i}, \stackrel{\circ}{\mathrm{D}}\left(R_{i}^{*}\right)\right)} \mathscr{L}(\boldsymbol{\gamma}, \boldsymbol{\psi})
$$

and the Entropy-Transport Problem can be written as

$$
\mathrm{ET}\left(\mu_{1}, \mu_{2}\right)=\inf _{\boldsymbol{\gamma} \in \mathrm{M}} \sup _{\psi_{i} \in \mathrm{LSC}_{s}\left(X_{i}, \mathrm{D}^{(}\left(R_{i}^{*}\right)\right)} \mathscr{L}(\boldsymbol{\gamma}, \boldsymbol{\psi}) .
$$

The dual problem can be obtained by interchanging the order of inf and sup as in Sect. 2.2. Let us denote by $\varphi_{1} \oplus \varphi_{2}$ the function $\left(x_{1}, x_{2}\right) \mapsto \varphi_{1}\left(x_{1}\right)+$ $\varphi_{2}\left(x_{2}\right)$. Since for every $\psi=\left(\psi_{1}, \psi_{2}\right)$ with $\psi_{i} \in \operatorname{LSC}_{s}\left(X_{i}, \stackrel{\circ}{\mathrm{D}}\left(R_{i}^{*}\right)\right)$,

$$
\begin{aligned}
& \inf _{\gamma \in \mathrm{M}} \int\left(\mathrm{c}\left(x_{1}, x_{2}\right)-R_{1}^{*}\left(\psi_{1}\left(x_{1}\right)\right)-R_{2}^{*}\left(\psi_{2}\left(x_{2}\right)\right)\right) \mathrm{d} \gamma \\
& \quad= \begin{cases}0 & \text { if } R_{1}^{*}\left(\psi_{1}\right) \oplus R_{2}^{*}\left(\psi_{2}\right) \leq \mathrm{c}, \\
-\infty & \text { otherwise, }\end{cases}
\end{aligned}
$$

we obtain

$$
\inf _{\boldsymbol{\gamma} \in \mathrm{M}} \mathscr{L}(\boldsymbol{\gamma}, \boldsymbol{\psi})= \begin{cases}\sum_{i} \int_{X_{i}} \psi_{i} \mathrm{~d} \mu_{i} & \text { if } R_{1}^{*}\left(\psi_{1}\right) \oplus R_{2}^{*}\left(\psi_{2}\right) \leq \mathrm{c} \\ -\infty & \text { otherwise. }\end{cases}
$$

Thus, (4.6) provides the dual formulation, that we will study in the next section. 


\subsection{Dual problem and optimality conditions}

Problem 4.1 ( $\psi$-formulation of the dual problem) Let $R_{i}^{*}$ be the convex functions defined by (4.1) and let $\boldsymbol{\Psi}$ be the convex set

$$
\boldsymbol{\Psi}:=\left\{\psi \in \operatorname{LSC}_{s}\left(X_{1}, \stackrel{\circ}{\mathrm{D}}\left(R_{1}^{*}\right)\right) \times \operatorname{LSC}_{s}\left(X_{2}, \stackrel{\circ}{\mathrm{D}}\left(R_{2}^{*}\right)\right): R_{1}^{*}\left(\psi_{1}\right) \oplus R_{2}^{*}\left(\psi_{2}\right) \leq \mathrm{c}\right\}
$$

The dual Entropy-Transport problem consists in finding a maximizer $\boldsymbol{\psi} \in \mathbf{\Psi}$ for

$$
\mathrm{D}\left(\mu_{1}, \mu_{2}\right)=\sup _{\boldsymbol{\psi} \in \boldsymbol{\Psi}} \int_{X_{1}} \psi_{1} \mathrm{~d} \mu_{1}+\int_{X_{2}} \psi_{2} \mathrm{~d} \mu_{2} .
$$

As usual, by the following change of variable

$$
\varphi_{i}:=R^{*}\left(\psi_{i}\right), \quad \psi_{i}=F_{i}^{\circ}\left(\varphi_{i}\right):=-F_{i}^{*}\left(-\varphi_{i}\right),
$$

as in (2.45) for the duality Theorem 2.7 (recall the notation $\phi_{i}=-\varphi_{i}$ we used in Sect. 2.3), we can obtain an equivalent formulation of the dual functional $\mathrm{D}$ as the supremum of the concave functionals

$$
\mathscr{D}\left(\varphi \mid \mu_{1}, \mu_{2}\right):=\sum_{i} \int_{X_{i}} F_{i}^{\circ}\left(\varphi_{i}\right) \mathrm{d} \mu_{i}
$$

on the simpler convex set

$$
\boldsymbol{\Phi}:=\left\{\varphi \in \operatorname{LSC}_{s}\left(X_{1}, \stackrel{\circ}{\mathrm{D}}\left(F_{1}^{\circ}\right)\right) \times \operatorname{LSC}_{s}\left(X_{2}, \stackrel{\circ}{\mathrm{D}}\left(F_{2}^{\circ}\right)\right), \varphi_{1} \oplus \varphi_{2} \leq \mathrm{c}\right\} .
$$

Problem 4.2 ( $\varphi$-formulation of the dual problem) Let $F_{i}^{\circ}$ be the concave functions defined by (4.9) and let $\boldsymbol{\Phi}$ be the the convex set (4.11). The $\varphi$-formulation of the dual Entropy-Transport problem consists in finding a maximizer $\boldsymbol{\varphi} \in \boldsymbol{\Phi}$ for

$$
\mathrm{D}^{\prime}\left(\mu_{1}, \mu_{2}\right)=\sup _{\boldsymbol{\varphi} \in \boldsymbol{\Phi}} \mathscr{D}\left(\varphi \mid \mu_{1}, \mu_{2}\right)=\sup _{\boldsymbol{\varphi} \in \boldsymbol{\Phi}} \sum_{i} \int_{X_{i}} F_{i}^{\circ}\left(\varphi_{i}\right) \mathrm{d} \mu_{i} .
$$

Proposition 4.3 (Equivalence of the dual formulations) The $\psi$ - and the $\phi$ formulations of the dual problem are equivalent, $\mathrm{D}\left(\mu_{1}, \mu_{2}\right)=\mathrm{D}^{\prime}\left(\mu_{1}, \mu_{2}\right)$.

Proof Let us first notice that replacing $\psi_{i}$ with $\psi_{i}-\varepsilon, \varepsilon>0$, and using the strict monotonicity of $R_{i}^{*}$ in $\left(\operatorname{aff}\left(F_{i}\right)_{\infty}, F_{i}(0)\right)$, as well as the fact that 
$R_{i}^{*} \equiv-\left(F_{i}\right)_{\infty}^{\prime}$ in $\left(-\infty, \operatorname{aff}\left(F_{i}\right)_{\infty}\right)$ and inf c $>-\left(F_{1}\right)_{\infty}^{\prime}-\left(F_{2}\right)_{\infty}^{\prime}$, one can replace $\boldsymbol{\Psi}$ in (4.8) by the smaller set

$$
\Psi^{\circ}:=\left\{\psi \in \operatorname{LSC}_{s}\left(X_{1}, \stackrel{\circ}{\mathrm{D}}\left(R_{1}^{*}\right)\right) \times \operatorname{LSC}_{s}\left(X_{2}, \stackrel{\circ}{\mathrm{D}}\left(R_{2}^{*}\right)\right): R_{1}^{*}\left(\psi_{1}\right) \oplus R_{2}^{*}\left(\psi_{2}\right)<\mathrm{c}\right\} .
$$

Since $R_{i}^{*}$ is nondecreasing, for every $\boldsymbol{\psi} \in \boldsymbol{\Psi}^{\circ}$ the functions $\varphi_{i}:=R_{i}^{*}\left(\psi_{i}\right)+\delta$, where $\delta:=\frac{1}{2} \inf \mathrm{C}-R_{1}^{*}\left(\psi_{1}\right) \oplus R_{2}^{*}\left(\psi_{2}\right)>0$, belong to $\operatorname{LSC}_{s}\left(X_{i}, \stackrel{\circ}{\mathrm{D}}\left(R_{i}^{*}\right)\right)$ and satisfy $\varphi_{1} \oplus \varphi_{2}<\mathrm{C}$, with $\left(-\varphi_{i}, \psi_{i}\right) \in \mathfrak{\mathfrak { F }}_{i}$. It then follows that $\left(\varphi_{1}, \varphi_{2}\right) \in \boldsymbol{\Phi}$ and $\tilde{\psi}_{i}:=-F_{i}^{*}\left(-\varphi_{i}\right)=F_{i}^{\circ}\left(\varphi_{i}\right) \geq \psi_{i}$ so that $\mathbf{D}^{\prime} \geq \mathrm{D}$. An analogous argument shows the converse inequality.

Since "inf sup $\geq$ sup inf" (cf. (2.10)), our derivation via (4.5) yields

$$
\mathrm{ET}\left(\mu_{1}, \mu_{2}\right) \geq \mathrm{D}\left(\mu_{1}, \mu_{2}\right) .
$$

Using Theorem 2.4 we will show in Sect. 4.3 that (4.13) is in fact an equality. Before this, we first discuss for which class of functions $\psi_{i}, \varphi_{i}$ the dual formulations are still meaningful. Moreover, we analyze the optimality conditions associated to the equality case in (4.13).

Extension to Borel functions. In some cases we will also consider larger classes of potentials $\psi$ or $\varphi$ by allowing Borel functions with extended real values, under suitable summability conditions. It is clear that in the formulation of a dual problem it can be useful to deal with a smaller set of "competitors" (as in Problem 4.1 where we consider simple and lower semicontinuous functions) to derive various properties by exploiting the specific features of the involved functions. On the other hand, when one aims to prove the existence of dual optimizers, it is natural to enlarge the set of competitors in order to gain better closure properties. This is one of the main motivation to extend the dual formulation to general Borel functions.

First of all, recalling (2.19) and (2.29), we extend $R^{*}$ and $F^{\circ}$ to $\overline{\mathbb{R}}$ by setting

$$
\begin{array}{ll}
R^{*}(-\infty):=-F_{\infty}^{\prime}, & R^{*}(+\infty):=+\infty \\
F^{\circ}(-\infty):=-\infty, & F^{\circ}(+\infty):=F(0),
\end{array}
$$

and we observe that, with the definition above and according to (2.38)-(2.39), the pairs

$$
\left(-\varphi, F^{\circ}(\varphi)\right) \text { and }\left(-R^{*}(\psi), \psi\right) \text { lie in } \overline{\mathfrak{F}} \text { if } \psi \leq F(0) \text { and } \varphi \geq-F_{\infty}^{\prime} .
$$

We also set 


$$
\zeta_{1}+{ }_{o} \zeta_{2}:=\lim _{n \rightarrow \infty}\left(-n \vee \zeta_{1} \wedge n\right)+\left(-n \vee \zeta_{2} \wedge n\right) \text { for every } \zeta_{1}, \zeta_{2} \in \overline{\mathbb{R}}
$$

Notice that $( \pm \infty)+_{o}( \pm \infty)= \pm \infty$ and in the ambiguous case $+\infty-\infty$ this definition yields $(+\infty)+_{o}(-\infty)=0$. We correspondingly extend the definition of $\oplus$ by setting

$$
\left(\zeta_{1} \oplus_{o} \zeta_{2}\right)\left(x_{1}, x_{2}\right):=\zeta_{1}\left(x_{1}\right)+{ }_{o} \zeta_{2}\left(x_{2}\right) \text { for every } \zeta_{i} \in \mathrm{B}\left(X_{i} ; \overline{\mathbb{R}}\right)
$$

The following result is the natural extension of Lemma 2.6, stating that $\mathscr{E}\left(\boldsymbol{\gamma} \mid \mu_{1}, \mu_{2}\right) \geq \mathscr{D}\left(\varphi \mid \mu_{1}, \mu_{2}\right)$ for a larger class of $\boldsymbol{\gamma}$ and $\boldsymbol{\varphi}$ than before.

Proposition 4.4 (Dual lower bound for extended real valued potentials) Let $\gamma$ be a feasible plan and let $\varphi \in \mathrm{B}\left(X_{1} ; \overline{\mathbb{R}}\right) \times \mathrm{B}\left(X_{2} ; \overline{\mathbb{R}}\right)$ satisfy $\varphi_{i} \geq-\left(F_{i}\right)_{\infty}^{\prime}$, $\varphi_{1} \oplus_{o} \varphi_{2} \leq \mathrm{C}$, and $\left(F_{i}^{\circ} \circ \varphi_{i}\right)_{-} \in \mathrm{L}^{1}\left(X_{i}, \mu_{i}\right)\left(\operatorname{resp} .\left(\varphi_{i}\right)_{+} \in \mathrm{L}^{1}\left(X_{i}, \gamma_{i}\right)\right)$.

Then we have $\left(\varphi_{i}\right)_{-} \in \mathrm{L}^{1}\left(X_{i} ; \gamma_{i}\right)\left(\right.$ resp. $\left.\left(F_{i}^{\circ} \circ \varphi_{i}\right)_{+} \in \mathrm{L}^{1}\left(X_{i}, \mu_{i}\right)\right)$ and

$$
\mathscr{E}\left(\boldsymbol{\gamma} \mid \mu_{1}, \mu_{2}\right) \geq \sum_{i} \int_{X_{i}} F_{i}^{\circ}\left(\varphi_{i}\right) \mathrm{d} \mu_{i} .
$$

Remark 4.5 In a similar way, if $\psi \in \mathrm{B}\left(X_{1}, \overline{\mathbb{R}}\right) \times \mathrm{B}\left(X_{2}, \overline{\mathbb{R}}\right)$ with $\psi_{i} \leq F_{i}(0)$, $R_{1}^{*}\left(\psi_{1}\right) \oplus_{o} R_{2}^{*}\left(\psi_{2}\right) \leq \mathrm{c}$, and $\left(\psi_{i}\right)_{-} \in \mathrm{L}^{1}\left(X_{i}, \mu_{i}\right)$ (resp. $\left(R_{i}^{*} \circ \psi_{i}\right)_{+} \in$ $\left.\mathrm{L}^{1}\left(X_{i}, \gamma_{i}\right)\right)$, then $\left(R_{i}^{*} \circ \psi_{i}\right)_{-} \in \mathrm{L}^{1}\left(X_{i}, \gamma_{i}\right)\left(\operatorname{resp} .\left(\psi_{i}\right)_{+} \in \mathrm{L}^{1}\left(X_{i}, \mu_{i}\right)\right)$ with

$$
\mathscr{E}\left(\boldsymbol{\gamma} \mid \mu_{1}, \mu_{2}\right) \geq \sum_{i} \int_{X_{i}} \psi_{i} \mathrm{~d} \mu_{i}
$$

Proof Let us consider (4.18) in the case that $\left(F_{i}^{\circ} \circ \varphi_{i}\right)_{-} \in \mathrm{L}^{1}\left(X_{i}, \mu_{i}\right)$ (the calculations in the other cases, including (4.19), are completely analogous). Applying Lemma 2.6 (with $\psi_{i}:=F_{i}^{\circ} \circ \varphi_{i}$ and $\phi_{i}:=-\varphi_{i}$ ) and (2.40) we obtain $\left(\varphi_{i}\right)_{-} \in \mathrm{L}^{1}\left(X_{i}, \gamma_{i}\right)$ and then

$$
\begin{aligned}
\mathscr{E}\left(\boldsymbol{\gamma} \mid \mu_{1}, \mu_{2}\right) & =\sum_{i} \mathscr{F}_{i}\left(\gamma_{i} \mid \mu_{i}\right)+\int_{X} \mathrm{c} \mathrm{d} \boldsymbol{\gamma} \\
& \geq \sum_{i} \mathscr{F}_{i}\left(\gamma_{i} \mid \mu_{i}\right)+\int_{X}\left(\varphi_{1}\left(x_{1}\right)+{ }_{o} \varphi_{2}\left(x_{2}\right)\right) \mathrm{d} \boldsymbol{\gamma} \\
& \geq \sum_{i} \mathscr{F}_{i}\left(\gamma_{i} \mid \mu_{i}\right)+\int_{X_{i}} \varphi_{i} \mathrm{~d} \gamma_{i} \stackrel{(2.41)}{\geq} \sum_{i} \int_{X_{i}} F_{i}^{\circ}\left(\varphi_{i}\right) \mathrm{d} \mu_{i} .
\end{aligned}
$$


Notice that the integrability of the negative part of $\varphi_{i}$ w.r.t. $\gamma_{i}$ yields $\varphi_{i}\left(\pi^{i}\left(x_{1}, x_{2}\right)\right)>-\infty$ for $\boldsymbol{\gamma}$-a.e. $\left(x_{1}, x_{2}\right) \in \boldsymbol{X}$ so that $\varphi_{1}\left(x_{1}\right)+_{o} \varphi_{2}\left(x_{2}\right)=$ $\varphi_{1}\left(x_{1}\right)+\varphi_{2}\left(x_{2}\right)$ and we can split the integral

$$
+\infty>\int\left(\sum_{i} \varphi_{i}\left(x_{i}\right)\right) \mathrm{d} \boldsymbol{\gamma}=\sum_{i} \int \varphi_{i}\left(x_{i}\right) \mathrm{d} \boldsymbol{\gamma}=\sum_{i} \int \varphi_{i}\left(x_{i}\right) \mathrm{d} \gamma_{i} .
$$

Optimality conditions. If there exists a pair $\varphi$ as in Proposition 4.4 such that $\mathscr{E}\left(\boldsymbol{\gamma} \mid \mu_{1}, \mu_{2}\right)=\mathscr{D}\left(\varphi \mid \mu_{1}, \mu_{2}\right)$ then all the above inequalities (4.20) should be identities so that we have

$$
\begin{aligned}
& \mathscr{F}_{i}\left(\gamma_{i} \mid \mu_{i}\right)=\int_{X_{i}} F_{i}^{\circ}\left(\varphi_{i}\right) \mathrm{d} \mu_{i}, \quad \text { and } \\
& \int_{X}\left(\mathrm{c}\left(x_{1}, x_{2}\right)-\left(\varphi_{1}\left(x_{1}\right)+{ }_{o} \varphi_{2}\left(x_{2}\right)\right)\right) \mathrm{d} \boldsymbol{\gamma}=0 .
\end{aligned}
$$

The second part of Lemma 2.6 then yields

$$
\begin{aligned}
\varphi_{1}\left(x_{1}\right)+_{o} \varphi_{2}\left(x_{2}\right) & =\mathrm{c}\left(x_{1}, x_{2}\right) \quad \boldsymbol{\gamma} \text {-a.e. in } \boldsymbol{X}, \\
-\varphi_{i} & \in \partial F_{i}\left(\sigma_{i}\right) \quad\left(\mu_{i}+\gamma_{i}\right) \text {-a.e. in } A_{i} \\
\varphi_{i} & =-\left(F_{i}\right)_{\infty}^{\prime} \quad \gamma_{i}^{\perp} \text {-a.e. in } A_{\gamma_{i}}, \\
F_{i}^{\circ}\left(\varphi_{i}\right) & =F_{i}(0) \quad \mu_{i}^{\perp} \text {-a.e. in } A_{\mu_{i}},
\end{aligned}
$$

where $\left(A_{i}, A_{\mu_{i}}, A_{\gamma_{i}}\right)$ is a Borel partition related to the Lebesgue decomposition of the pair $\left(\gamma_{i}, \mu_{i}\right)$ as in Lemma 2.3. We will show now that the existence of a pair $\varphi$ satisfying

$$
\boldsymbol{\varphi}=\left(\varphi_{1}, \varphi_{2}\right) \in \mathrm{B}\left(X_{1} ; \overline{\mathbb{R}}\right) \times \mathrm{B}\left(X_{2} ; \overline{\mathbb{R}}\right), \quad \varphi_{i} \geq-\left(F_{i}\right)_{\infty}^{\prime}, \quad \varphi_{1} \oplus_{o} \varphi_{2} \leq \mathrm{c},
$$

and the joint optimality conditions 4.21 is also sufficient to prove that a feasible $\boldsymbol{\gamma} \in \mathcal{M}(\boldsymbol{X})$ is optimal. We emphasize that we do not need any integrability assumption on $\varphi$.

Theorem 4.6 Let us suppose that Problem 3.1 is feasible (see Remark 3.2) for $\mu_{i} \in \mathcal{M}\left(X_{i}\right)$ and let $\boldsymbol{\gamma} \in \mathcal{M}(\boldsymbol{X})$; if there exists a pair $\boldsymbol{\varphi}$ as in (4.22) that satisfies the joint optimality conditions (4.21), then $\gamma$ is optimal.

Proof We want to repeat the calculations in (4.20) of Proposition 4.4, but now taking care of the integrability issues. We use a clever truncation argument of [43], based on the maps

$$
T_{n}: \mathbb{R} \rightarrow \mathbb{R}, \quad T_{n}(\varphi):=-n \vee \varphi \wedge n,
$$


combined with a corresponding approximations of the entropies $F_{i}$ given by

$$
F_{i, n}(r):=\max _{|\phi| \leq n}\left(\phi r-F_{i}^{*}(\phi)\right) .
$$

Recalling (4.16), it is not difficult to check that if $\varphi_{1}+_{o} \varphi_{2} \geq 0$ we have $0 \leq T_{n}\left(\varphi_{1}\right)+T_{n}\left(\varphi_{2}\right) \uparrow \varphi_{1}+\varphi_{2}$ as $n \uparrow \infty$, whereas $\varphi_{1}+_{o} \varphi_{2} \leq 0$ yields $0 \geq T_{n}\left(\varphi_{1}\right)+T_{n}\left(\varphi_{2}\right) \downarrow \varphi_{1}+\varphi_{2}$ (notice that the cases when $\varphi_{1}= \pm \infty$, $\varphi_{2}=\mp \infty$ correspond to $\left.T_{n}\left(\varphi_{1}\right)+T_{n}\left(\varphi_{2}\right)=\varphi_{1}+{ }_{o} \varphi_{2}=0 \leq \mathrm{C}\right)$.

In particular if $\varphi$ satisfies (4.22) then $T_{n}\left(\varphi_{i}\right) \in \mathrm{B}_{b}\left(X_{i}\right), T_{n}\left(\varphi_{1}\right) \oplus T_{n}\left(\varphi_{2}\right) \leq \mathrm{C}$, and $T_{n}\left(\varphi_{i}\right) \geq-\left(F_{i}\right)_{\infty}^{\prime}$ due to $\left(F_{i}\right)_{\infty}^{\prime} \geq 0$ and $\varphi_{i} \geq-\left(F_{i}\right)_{\infty}^{\prime}$. The boundedness of $T_{n}\left(\varphi_{i}\right)$ and Proposition 4.4 yield for every $\tilde{\boldsymbol{\gamma}} \in \mathcal{M}(\boldsymbol{X})$

$$
\mathscr{E}\left(\tilde{\gamma} \mid \mu_{1}, \mu_{2}\right) \geq \sum_{i} \int_{X_{i}} F_{i}^{\circ}\left(T_{n}\left(\varphi_{i}\right)\right) \mathrm{d} \mu_{i} .
$$

When $\left(F_{i}\right)_{\infty}^{\prime}<\infty$, choosing $n \geq\left(F_{i}\right)_{\infty}^{\prime}$ so that $T_{n}\left(\varphi_{i}\right)=\varphi_{i}=-\left(F_{i}\right)_{\infty}^{\prime}$ $\gamma_{i}^{\perp}$-a.e., and applying (ii) of the next Lemma 4.7, we obtain

$$
\begin{aligned}
& \int_{X_{i}} F_{i}^{\circ}\left(T_{n}\left(\varphi_{i}\right)\right) \mathrm{d} \mu_{i} \stackrel{(4.21 \mathrm{~b}, \mathrm{~d})}{=} \int_{X_{i}}\left(F_{i, n}\left(\sigma_{i}\right)+\sigma_{i} T_{n}\left(\varphi_{i}\right)\right) \mathrm{d} \mu_{i} \\
& \stackrel{(4.21 \mathrm{c})}{=} \int_{X_{i}} F_{i, n}\left(\sigma_{i}\right) \mathrm{d} \mu_{i}+\left(F_{i}\right)_{\infty}^{\prime} \gamma_{i}^{\perp}\left(X_{i}\right)+\int_{X_{i}} T_{n}\left(\varphi_{i}\right) \mathrm{d} \gamma_{i},
\end{aligned}
$$

and the same relation also holds when $\left(F_{i}\right)_{\infty}^{\prime}=+\infty$ since in this case $\gamma_{i}^{\perp}=0$. Summing up the two contributions we get

$$
\begin{aligned}
\mathscr{E}\left(\tilde{\gamma} \mid \mu_{1}, \mu_{2}\right) \geq & \sum_{i}\left(\int_{X_{i}} F_{i, n}\left(\sigma_{i}\right) \mathrm{d} \mu_{i}+\left(F_{i}\right)_{\infty}^{\prime} \gamma_{i}^{\perp}\left(X_{i}\right)\right) \\
& +\int_{X}\left(T_{n}\left(\varphi_{1}\right) \oplus T_{n}\left(\varphi_{2}\right)\right) \mathrm{d} \boldsymbol{\gamma} .
\end{aligned}
$$

Applying Lemma 4.7 (i) and the fact that $\varphi_{1} \oplus_{o} \varphi_{2}=\mathrm{c} \geq 0 \boldsymbol{\gamma}$-a.e. by (4.21a), we can pass to the limit as $n \uparrow \infty$ by monotone convergence in the right-hand side, obtaining the desired optimality $\mathscr{E}\left(\tilde{\gamma} \mid \mu_{1}, \mu_{2}\right) \geq \mathscr{E}\left(\boldsymbol{\gamma} \mid \mu_{1}, \mu_{2}\right)$.

Lemma 4.7 Let $F_{i, n}:[0, \infty) \rightarrow[0, \infty)$ be defined by (4.24). Then

(i) $F_{i, n}$ are Lipschitz, $F_{i, n}(s) \leq F_{i}(s)$, and $F_{i, n}(s) \uparrow F_{i}(s)$ as $n \uparrow \infty$.

(ii) For every $s \in \mathrm{D}\left(F_{i}\right)$ and $\varphi_{i} \in \mathbb{R} \cup\{+\infty\}$ we have

$$
\begin{aligned}
-\varphi_{i} \in \partial F_{i}(s) & \Rightarrow-T_{n}\left(\varphi_{i}\right) \in \partial F_{i, n}(s), \\
\varphi_{i}=+\infty, s=0 & \Rightarrow \quad F_{i, n}(0)=F_{i}^{\circ}\left(T_{n}\left(\varphi_{i}\right)\right)=F_{i}^{\circ}(n) .
\end{aligned}
$$


In particular, both cases in (4.26) give $F_{i}^{\circ}\left(T_{n}\left(\varphi_{i}\right)\right)=F_{i, n}(s)+s T_{n}\left(\varphi_{i}\right)$.

Proof Property (i): By (2.23) and the definition in (4.24) we get $F_{i, n} \leq F_{i}$. Since $-F_{i}^{*}(0)=\inf F_{i} \geq 0$ we see that $F_{i, n}$ are nonnegative. Recalling that $F_{i}^{*}$ are nondecreasing with $\mathrm{D}\left(F_{i}^{*}\right) \supset(-\infty, 0)$ (see (2.18)), we also get the upper bound $F_{i, n}(s) \leq-n s-F_{i}^{*}(-n)$. Eventually, (4.24) defines $F_{i, n}$ as the maximum of a family of $n$-Lipschitz functions, so $F_{i, n}$ is $n$-Lipschitz.

Property (ii): Let us set $F_{i, n}^{*}:=F_{i}^{*}+\mathrm{I}_{[-n, n]}$ and notice that $F_{i, n}=\left(F_{i, n}^{*}\right)^{*}$ so that $\left(F_{i, n}\right)^{*}=F_{i, n}^{*}$. Recalling that $\left(\partial F_{i}\right)^{-1}=\partial F_{i}^{*},\left(\partial F_{i, n}\right)^{-1}=\partial F_{i, n}^{*}$, $\partial F_{i}^{*}+\partial \mathrm{I}_{[-n, n]} \subset \partial F_{i, n}^{*}$ and

$$
\partial \mathrm{I}_{[-n, n]}(\phi)= \begin{cases}0 & \text { if } \phi \in(-n, n), \\ {[0, \infty)} & \text { if } \phi=n, \\ (-\infty, 0] & \text { if } \phi=-n, \\ \varnothing & \text { if }|\phi|>n,\end{cases}
$$

we can easily prove the first implication of (4.26). In fact $-\varphi_{i} \in \partial F_{i}(s)$ yields $s \in \partial F_{i}^{*}\left(-\varphi_{i}\right)$ and $s=T_{n}(s) \in \partial F_{i, n}^{*}\left(-\varphi_{i}\right)$ if $\left|\varphi_{i}\right| \leq n$; when $-\varphi_{i}>n$ the monotonicity of the subdifferential and the fact that $n \in \mathrm{D}\left(F^{*}\right)_{i} \subset \mathrm{D}\left(\partial F_{i}^{*}\right)$ yields $s \geq s^{\prime}$ for every $s^{\prime} \in \partial F_{i}(n)$ so that $s \in \partial F_{i}^{*}+[0, \infty) \subset \partial F_{i, n}^{*}$. A similar argument holds when $-\varphi_{i}<-n$.

Eventually, if $\phi_{i}=-\infty$ and $s=0$ (in particular $F_{i}(0)=F_{i}^{*}(-\infty)<\infty$ ), then (4.24) and the fact that $F_{i}^{*}$ is nondecreasing yields $F_{i, n}(0)=-F_{i}^{*}(-n)=$ $F_{i}^{\circ}(n)=F_{i}^{\circ}\left(T_{n}\left(\varphi_{i}\right)\right)$.

The last statement in (ii) is an immediate application of (4.26) and the link between subdifferential and Fenchel duality stated in (2.17).

\subsection{A general duality result}

The aim of this section is to show in complete generality the duality result $E T=D$, by using the $\varphi$-formulation of the dual problem (4.12), which is equivalent to (4.7) by Proposition 4.3.

We start with a simple lemma depending on a specific feature of the entropy functions (which fails exactly in the case of pure transport problems, see Example E.3 of Sect. 3.3), using the strengthened feasibility condition in (3.12). First note that the pair $\varphi_{i} \equiv 0$ provides an obvious lower bound for $\mathrm{D}\left(\mu_{1}, \mu_{2}\right)$, viz.

$$
\mathrm{D}\left(\mu_{1}, \mu_{2}\right) \geq \mathscr{D}\left(0,0 \mid \mu_{1}, \mu_{2}\right)=\sum_{i} m_{i} F_{i}^{\circ}(0)=\sum_{i} m_{i} \inf F_{i} .
$$

We derive an upper and lower bound for the potential $\varphi_{1}$ under the assumption that $\mathrm{C}$ is bounded. 
Lemma 4.8 Let $m_{i}=\mu_{i}\left(X_{i}\right)$ and assume int $\left(m_{1} \mathrm{D}\left(F_{1}\right)\right) \cap m_{2} \mathrm{D}\left(F_{2}\right) \neq \emptyset$, so that

$$
\exists s_{1}^{-}, s_{1}^{+} \in \mathrm{D}\left(F_{1}\right), s_{2} \in \mathrm{D}\left(F_{2}\right): \quad m_{1} s_{1}^{-}<m_{2} s_{2}<m_{1} s_{1}^{+},
$$

and $S:=\sup \mathrm{C}<\infty$. Then every pair $\boldsymbol{\varphi}=\left(\varphi_{1}, \varphi_{2}\right) \in \boldsymbol{\Phi}$ with $\mathscr{D}\left(\boldsymbol{\varphi} \mid \mu_{1}, \mu_{2}\right) \geq$ $\sum_{i} m_{i} \inf F_{i}$ satisfies

$$
\begin{aligned}
& \Phi_{1}^{-} \leq \sup \varphi_{1} \leq \Phi_{1}^{+}, \\
& \Phi_{1}^{ \pm}:=\frac{m_{1}\left(F_{1}\left(s_{1}^{ \pm}\right)-\inf F_{1}\right)+m_{2}\left(F_{2}\left(s_{2}\right)-\inf F_{2}\right)+m_{2} s_{2} S}{m_{2} s_{2}-m_{1} s_{1}^{ \pm}} .
\end{aligned}
$$

Proof Since $\boldsymbol{\varphi}=\left(\varphi_{1}, \varphi_{2}\right) \in \boldsymbol{\Phi}$ satisfies $\sup \varphi_{1}+\sup \varphi_{2} \leq S$, the definition of $\mathscr{D}$ in (4.10) and the monotonicity of $F^{\circ}$ yield

$$
\sum_{i} m_{i} \inf F_{i} \leq \mathscr{D}\left(\varphi \mid \mu_{1}, \mu_{2}\right) \leq m_{1} F_{1}^{\circ}\left(\sup \varphi_{1}\right)+m_{2} F_{2}^{\circ}\left(S-\sup \varphi_{1}\right)
$$

Using the dual bound $F_{i}^{\circ}\left(\varphi_{i}\right) \leq \varphi_{i} s_{i}+F_{i}\left(s_{i}\right)$ for $s_{i} \in \mathrm{D}\left(F_{i}\right)$ (cf. (4.9)) now implies

$$
\begin{aligned}
\sum_{i} m_{i} \text { inf } F_{i} & \leq \mathscr{D}\left(\varphi \mid \mu_{1}, \mu_{2}\right) \\
& \leq\left(m_{1} s_{1}-m_{2} s_{2}\right) \sup \varphi_{1}+m_{1} F_{1}\left(s_{1}\right)+m_{2} F_{2}\left(s_{2}\right)+m_{2} s_{2} S .
\end{aligned}
$$

Exploiting (4.28), the choice $s_{1}:=s_{1}^{-}$shows the upper bound in (4.29); and $s_{1}=s_{1}^{+}$the lower bound.

We improve the previous result by showing that in the case of bounded cost functions it is sufficient to consider bounded potentials $\varphi_{i}$. This lemma is well known in the case of Optimal Transport problems and will provide a useful a priori estimate in the case of bounded cost functions; it will also play an important role in the third step of the proof of Theorem 4.11, which contains the main result concerning the dual representation.

Lemma 4.9 If $\sup c=S<\infty$, then for every pair $\boldsymbol{\varphi} \in \boldsymbol{\Phi}$, there exists $\tilde{\boldsymbol{\varphi}} \in \boldsymbol{\Phi}$ such that $\mathscr{D}\left(\tilde{\boldsymbol{\varphi}} \mid \mu_{1}, \mu_{2}\right) \geq \mathscr{D}\left(\varphi \mid \mu_{1}, \mu_{2}\right)$ and

$$
\sup \tilde{\varphi}_{i}-\inf \tilde{\varphi}_{i} \leq S, \quad 0 \leq \sup \tilde{\varphi}_{1}+\sup \tilde{\varphi}_{2} \leq S .
$$

If moreover (3.12) holds, than there exist a constant $\varphi_{\max } \geq 0$ only depending on $F_{i}, m_{i}, S$ such that

$$
-\varphi_{\max } \leq \inf \tilde{\varphi}_{i} \leq \sup \tilde{\varphi}_{i} \leq \varphi_{\max } .
$$


Proof Since $\mathrm{C} \geq 0$, possibly replacing $\varphi_{1}$ with $\tilde{\varphi}_{1}:=\varphi_{1} \vee\left(-\sup \varphi_{2}\right)$ we obtain a new pair $\left(\tilde{\varphi}_{1}, \varphi_{2}\right)$ with

$$
\tilde{\varphi}_{1} \geq \varphi_{1}, \quad \tilde{\varphi}_{1}\left(x_{1}\right)+\varphi_{2}\left(x_{2}\right) \leq\left(\varphi_{1}\left(x_{1}\right)+\varphi_{2}\left(x_{2}\right)\right) \wedge 0 \leq \mathrm{c}\left(x_{1}, x_{2}\right)
$$

so that $\left(\tilde{\varphi}_{1}, \varphi_{2}\right) \in \boldsymbol{\Phi}$ and $\mathscr{D}\left(\tilde{\varphi}_{1}, \varphi_{2} \mid \mu_{1}, \mu_{2}\right) \geq \mathscr{D}\left(\varphi_{1}, \varphi_{2} \mid \mu_{1}, \mu_{2}\right)$ since $F_{1}^{\circ}$ is nondecreasing. It is then not restrictive to assume that inf $\varphi_{1} \geq-\sup \varphi_{2}$; a similar argument shows that we can assume $\inf \varphi_{2} \geq-\sup \varphi_{1}$. Since

$$
\sup \varphi_{1}+\sup \varphi_{2} \leq S
$$

we thus obtain a new pair $\left(\tilde{\varphi}_{1}, \tilde{\varphi}_{2}\right) \in \Sigma$ with

$$
\mathscr{D}\left(\tilde{\varphi}_{1}, \tilde{\varphi}_{2} \mid \mu_{1}, \mu_{2}\right) \geq \mathscr{D}\left(\varphi_{1}, \varphi_{2} \mid \mu_{1}, \mu_{2}\right), \quad \sup \tilde{\varphi}_{i}-\inf \tilde{\varphi}_{i} \leq S .
$$

If moreover $\sup \varphi_{1}+\sup \varphi_{2}=-\delta<0$, we could always add the constant $\delta$ to, e.g., $\varphi_{1}$, thus increasing the value of $\mathscr{D}$ while still preserving the constraint $\boldsymbol{\Phi}$. Thus, (4.30) is established.

When (3.12) holds (e.g. in the case considered by (4.28)) the previous Lemma 4.8 provides constants $\varphi_{1}^{ \pm}$such that $\varphi_{1}^{-} \leq \sup \tilde{\varphi}_{1} \leq \varphi_{1}^{+}$. Now, (4.30) shows that $\varphi_{2}^{-} \leq \sup \tilde{\varphi}_{2} \leq \varphi_{2}^{+}$with $\varphi_{2}^{-}:=-\varphi_{1}^{+}$and $\varphi_{2}^{+}:=S-\varphi_{1}^{-}$. Applying (4.30) once again, we obtain (4.31) with $\varphi_{\max }:=S+\varphi_{1}^{+}-\varphi_{1}^{-}$.

Before stating the last lemma we recall the useful notion of c-transforms of functions $\varphi_{i}: X_{i} \rightarrow \overline{\mathbb{R}}$ for a real valued cost c $: X \rightarrow[0, \infty)$, defined via

$\varphi_{1}^{\mathrm{C}}\left(x_{2}\right):=\inf _{x \in X_{1}}\left(\mathrm{c}\left(x, x_{2}\right)-\varphi_{1}(x)\right)$ and $\varphi_{2}^{\mathrm{C}}\left(x_{1}\right):=\inf _{x \in X_{2}}\left(\mathrm{c}\left(x_{1}, x\right)-\varphi_{2}(x)\right)$.

It is not difficult to show (see e.g. [2, Sect. 6.1]) that if $\varphi_{1} \oplus \varphi_{2} \leq \mathrm{C}$ with $\sup \varphi_{i}<\infty$ then

$$
\varphi_{1}^{\mathrm{C}} \text { and } \varphi_{2}^{\mathrm{c}} \text { are bounded, } \quad \varphi_{1}^{\mathrm{cc}} \oplus \varphi_{1}^{\mathrm{C}} \leq \mathrm{c}, \quad \varphi_{1}^{\mathrm{cc}} \geq \varphi_{1} \text {, and } \varphi_{1}^{\mathrm{c}} \geq \varphi_{2} .
$$

Moreover, $\varphi_{1}=\varphi_{1}^{\mathrm{CC}}$ if and only if $\varphi_{1}=\varphi_{2}^{\mathrm{C}}$ for some function $\varphi_{2}$; in this case $\varphi_{1}$ is called C-concave and $\left(\varphi_{1}^{\mathrm{CC}}, \varphi_{1}^{\mathrm{C}}\right)$ is a pair of C-concave potentials.

Since $F_{i}^{\circ}$ are nondecreasing, it is also clear that whenever $\varphi_{1}^{\mathrm{CC}}, \varphi_{1}^{\mathrm{C}}$ are $\mu_{i}$ measurable we have the estimate

$$
\begin{aligned}
\forall \varphi \in \mathrm{B}\left(X_{1}\right) \times \mathrm{B}\left(X_{2}\right), & \varphi_{1} \oplus \varphi_{2} \leq \mathrm{c}: \\
& \mathscr{D}\left(\left(\varphi_{1}, \varphi_{2}\right) \mid \mu_{1}, \mu_{2}\right) \leq \mathscr{D}\left(\left(\varphi_{1}^{\mathrm{cc}}, \varphi_{2}^{\mathrm{c}}\right) \mid \mu_{1}, \mu_{2}\right)
\end{aligned}
$$

The next lemma concerns the lower semicontinuity of $\varphi_{i}^{\mathrm{C}}$ in the case when $\mathrm{C}$ has the particular form (cf. [26]) 


$$
\mathrm{c}=\sum_{n=1}^{N} c_{n} \chi_{A_{n}^{1} \times A_{n}^{2}}, \quad \text { with } c_{n} \geq 0 \text { and } A_{n}^{i} \text { open in } X_{i}
$$

Lemma 4.10 Let us assume that $\mathrm{c}$ has the form (4.37) and that $\varphi \in \mathrm{B}_{S}\left(X_{1}\right) \times$ $\mathrm{B}_{s}\left(X_{2}\right)$ is a pair of simple functions taking values in $\mathrm{D}\left(F_{1}^{\circ}\right) \times \mathrm{D}\left(F_{2}^{\circ}\right)$ and satisfying $\varphi_{1} \oplus \varphi_{2} \leq \mathrm{C}$. Then $\left(\varphi_{1}^{\mathrm{CC}}, \varphi_{1}^{\mathrm{C}}\right) \in \boldsymbol{\Phi}$ with $\mathscr{D}\left(\left(\varphi_{1}^{\mathrm{CC}}, \varphi_{1}^{\mathrm{C}}\right) \mid \mu_{1}, \mu_{2}\right) \geq$ $\mathscr{D}\left(\varphi \mid \mu_{1}, \mu_{2}\right)$.

Proof It is easy to check that $\varphi_{1}^{\mathrm{cC}}, \varphi_{1}^{\mathrm{C}}$ are simple, since the infima in (4.34) are taken on a finite number of possible values. By (4.35) it is thus sufficient to check that they are lower semicontinuous functions.

We do this for $\varphi_{1}^{\mathrm{C}}$, the argument for $\varphi_{1}^{\mathrm{cc}}=\left(\varphi_{1}^{\mathrm{C}}\right)^{\mathrm{C}}$ is completely analogous. For this, consider the sets

$$
\begin{aligned}
Z & :=\left\{z=\left(z_{n}\right)_{n=1}^{N} \in\{0,1\}^{N}: \exists y \in X_{1} \forall n=1, \ldots, N: z_{n}=\chi_{A_{n}^{1}}(y)\right\}, \\
Y_{z} & :=\left\{y \in X_{1}: \forall n=1, \ldots, N: \chi_{A_{n}^{1}}(y)=z_{n}\right\} .
\end{aligned}
$$

Clearly, $\left(Y_{z}\right)_{z \in Z}$ defines a Borel partition of $X_{1}$; we define $\varphi_{z}:=\sup \left\{\varphi_{1}(y)\right.$ : $\left.y \in Y_{z}\right\}$.

By construction, for every $z \in Z$ and $y \in Y_{z}$ the map $f_{z}(x):=\mathrm{c}(y, x)-\varphi_{z}$ is independent of $y$ in $Y_{z}$ and it is lower semicontinuous w.r.t. $x \in X_{2}$ since $\mathrm{C}$ is lower semicontinuous. Since $\varphi_{1}^{\mathrm{c}}\left(x_{2}\right)$ is the minimum of a finite collection of lower semicontinuous functions, viz.

$$
\varphi_{1}^{\mathrm{c}}\left(x_{2}\right)=\min \left\{f_{z}\left(x_{2}\right): z \in Z\right\}
$$

we obtain $\varphi_{1}^{\mathrm{C}} \in \operatorname{LSC}\left(X_{1}\right)$.

With all these auxiliary results at hand, we are now ready to prove our main result concerning the dual representation using Theorem 2.4.

Theorem 4.11 In the basic coercive setting of rm Sect. 3.1 (i.e. (3.2a) or (3.2b) hold), the Entropy-Transport functional (3.4) and the dual functional (4.10) satisfy

$$
\inf _{\boldsymbol{\gamma} \in \mathcal{M}\left(X_{1} \times X_{2}\right)} \mathscr{E}\left(\boldsymbol{\gamma} \mid \mu_{1}, \mu_{2}\right)=\sup _{\boldsymbol{\varphi} \in \boldsymbol{\Phi}} \mathscr{D}\left(\varphi \mid \mu_{1}, \mu_{2}\right) \text { for every } \mu_{i} \in \mathcal{M}\left(X_{i}\right) \text {, }
$$

i.e. $\mathrm{ET}\left(\mu_{1}, \mu_{2}\right)=\mathrm{D}\left(\mu_{1}, \mu_{2}\right)$ for every $\mu_{i} \in \mathcal{M}\left(X_{i}\right)$.

Proof Since ET $\geq \mathrm{D}$ is obvious, it suffices to show $\mathrm{ET} \leq \mathrm{D}$. In particular, it is not restrictive to assume that $\mathrm{D}\left(\mu_{1}, \mu_{2}\right)$ is finite. We proceed in various steps, considering first the case when $c$ has compact sublevels. Starting from Step 2 we will assume that $\left(F_{i}\right)_{\infty}^{\prime}=+\infty$ (so that $F_{i}^{\circ}$ are continuous and increasing 
on $\mathbb{R}$, and $F_{i}^{\circ} \circ \varphi_{i} \in \operatorname{LSC}_{b}\left(X_{i}\right)$ whenever $\left.\varphi_{i} \in \operatorname{LSC}_{b}\left(X_{i}\right)\right)$, and we will remove the compactness assumption on the sublevels of $\mathrm{C}$.

Step 1. We show that if the cost $\mathrm{c}$ has compact sublevels then (4.39) holds: We can directly apply Theorem 2.4 to the saddle functional $\mathscr{L}$ of (4.3) by choosing $A=\mathrm{M}$ given by (4.4) endowed with the narrow topology and $B=\operatorname{LSC}_{s}\left(X_{1}, \stackrel{\circ}{\mathrm{D}}\left(R_{1}^{*}\right)\right) \times \operatorname{LSC}_{s}\left(X_{1}, \stackrel{\circ}{\mathrm{D}}\left(R_{1}^{*}\right)\right)$. Conditions (2.9a) and (2.9b) are clearly satisfied; in order to check (2.11) we make use of the coercivity assumption $\left(F_{1}\right)_{\infty}^{\prime}+\left(F_{2}\right)_{\infty}^{\prime}+\min \mathrm{c}>0$ to find $\psi_{\star}=\left(\bar{\psi}_{1}, \bar{\psi}_{2}\right) \in B$ with constant functions $\bar{\psi}_{i} \in \stackrel{\circ}{\mathrm{D}}\left(R_{i}^{*}\right)$ and $-R^{*}\left(\bar{\psi}_{i}\right)=-\bar{\varphi}_{i}=\bar{\phi}_{i} \in\left(-\infty,\left(F_{i}\right)_{\infty}^{\prime}\right)$ such that

$$
D=\min \left(\mathrm{c}-\left(\bar{\varphi}_{1} \oplus \bar{\varphi}_{2}\right)\right)=\bar{\phi}_{1}+\bar{\phi}_{2}+\min \mathrm{c}>0 .
$$

Since

$$
\mathscr{L}\left(\boldsymbol{\gamma}, \boldsymbol{\psi}_{\star}\right)=\int_{\boldsymbol{X}}(\mathrm{c}-\min \mathrm{c}) \mathrm{d} \boldsymbol{\gamma}+D \boldsymbol{\gamma}(\boldsymbol{X})+\sum_{i} \bar{\psi}_{i} \mu_{i}\left(X_{i}\right),
$$

we immediately see that for $C$ sufficiently big the sublevels $\{\boldsymbol{\gamma} \in \mathrm{M}$ : $\left.\mathscr{L}\left(\boldsymbol{\gamma}, \boldsymbol{\psi}_{*}\right) \leq C\right\}$ are closed, bounded (since $D>0$ ) and equally tight (by the compactness of the sublevels of C), thus narrowly compact. Thus, (4.39), i.e. $E T=D$, follows from Theorem 2.4; this concludes the proof of Theorem 4.11 in the case when (3.2b) holds.

From now on we consider the case (3.2a), by assuming $F_{i}$ superlinear, i.e. $\left(F_{i}\right)_{\infty}^{\prime}=+\infty$.

Step 2. We show that if $\mu_{i}$ have compact support, if (3.12) is satisfied, and if the cost $\mathrm{C}$ has the form (4.37), then (4.39) holds: Let us set $\tilde{X}_{i}:=$ $\operatorname{supp}\left(\mu_{i}\right)$. Since $\left(F_{i}\right)_{\infty}^{\prime}=+\infty$ the support of all $\boldsymbol{\gamma}$ with $\mathscr{E}\left(\boldsymbol{\gamma} \mid \mu_{1}, \mu_{2}\right)<\infty$ is contained $\tilde{X}_{1} \times \tilde{X}_{2}$ so that the minimum of the functional $\mathscr{E}\left(\boldsymbol{\gamma} \mid \mu_{1}, \mu_{2}\right)$ does not change by restricting the spaces to $\tilde{X}_{i}$. By applying the previous step to the problem stated in $\tilde{X}_{1} \times \tilde{X}_{2}$, for every constant $E<\mathrm{ET}\left(\mu_{1}, \mu_{2}\right)$ we find $\boldsymbol{\varphi} \in \operatorname{LSC}_{s}\left(\tilde{X}_{1}\right) \times \operatorname{LSC}_{s}\left(\tilde{X}_{2}\right)$ such that $\varphi_{1} \oplus \varphi_{2} \leq \mathrm{c}$ in $\tilde{X}_{1} \times \tilde{X}_{2}$, that $F_{i}^{\circ}\left(\varphi_{i}\right)$ is finite, and that $\sum_{i} \int_{\tilde{X}_{i}} F_{i}^{\circ}\left(\varphi_{i}\right) \mathrm{d} \mu_{i} \geq E$.

Extending $\varphi_{i}$ to $-\sup \operatorname{cin} X_{i} \backslash \tilde{X}_{i}$ the value of $\mathscr{D}\left(\varphi \mid \mu_{1}, \mu_{2}\right)$ does not change and we obtain a pair of simple Borel functions with $\varphi_{1} \oplus \varphi_{2} \leq \mathrm{C}$ in $\boldsymbol{X}$. We can eventually apply Lemma 4.10 to find $\left(\varphi_{1}^{\mathrm{CC}}, \varphi_{1}^{\mathrm{C}}\right) \in \boldsymbol{\Phi}$ with $\mathscr{D}\left(\varphi_{1}^{\mathrm{CC}}, \varphi_{1}^{\mathrm{C}} \mid \mu_{1}, \mu_{2}\right) \geq$ $E$. Since $E<\mathrm{ET}\left(\mu_{1}, \mu_{2}\right)$ was arbitrary, we conclude that (4.39) holds in this case as well.

In the next step we remove the assumption on the compactness of $\operatorname{supp}\left(\mu_{i}\right)$.

Step 3. We show that if (3.12) is satisfied and if the cost $\mathrm{c}$ has the form (4.37), then (4.39) holds: Since $\mu_{i}$ are Radon, we find two sequences of compact sets 
$K_{i, n} \subset X_{i}$ such that $\varepsilon_{i, n}:=\mu_{i}\left(X_{i} \backslash K_{i, n}\right) \rightarrow 0$ as $n \rightarrow \infty$, i.e. $\mu_{i, n}:=$ $\chi_{K_{i, n}} \cdot \mu_{i}$ converges narrowly to $\mu_{i}$.

Let $E_{n}:=\operatorname{ET}\left(\mu_{1, n}, \mu_{2, n}\right)$ and let $E_{n}^{\prime}<E_{n}$ with $\lim _{n \rightarrow \infty} E_{n}^{\prime}=$ $\liminf _{n \rightarrow \infty} E_{n}$. Since $\mu_{i, n}$ have compact support, by the previous step and Lemma 4.9 we can find a sequence $\boldsymbol{\varphi}_{n} \in \boldsymbol{\Phi}$ and a constant $\varphi_{\max }$ independent of $n$ such that

$$
\mathscr{D}\left(\boldsymbol{\varphi}_{n} \mid \mu_{1, n}, \mu_{2, n}\right) \geq E_{n}^{\prime} \text { and } \sup \left|\varphi_{n}^{i}\right| \leq \varphi_{\max }
$$

This yields

$$
\begin{aligned}
\mathscr{D}\left(\varphi_{n} \mid \mu_{1}, \mu_{2}\right) & \geq \sum_{i} \int_{K_{i, n}} F_{i}^{\circ}\left(\varphi_{i, n}\right) \mathrm{d} \mu_{i}+\sum_{i} F_{i}^{\circ}\left(-\varphi_{\max }\right) \varepsilon_{i, n} \\
& \geq E_{n}^{\prime}+\sum_{i} F_{i}^{\circ}\left(-\varphi_{\max }\right) \varepsilon_{i, n} .
\end{aligned}
$$

Using the lower semicontinuity of ET from Lemma 3.9 we obtain

$$
\begin{aligned}
& \mathrm{D}\left(\mu_{1}, \mu_{2}\right) \geq \liminf _{n \rightarrow \infty} \mathscr{D}\left(\boldsymbol{\varphi}_{n} \mid \mu_{1}, \mu_{2}\right) \geq \lim _{n \rightarrow \infty} E_{n}^{\prime} \\
& =\liminf _{n \rightarrow \infty} \mathrm{ET}\left(\mu_{1, n}, \mu_{2, n}\right) \geq \mathrm{ET}\left(\mu_{1}, \mu_{2}\right) .
\end{aligned}
$$

Thus, (4.39) is established.

In the next step we remove the assumption (3.12) on $F_{i}$.

Step 4. We show that if the cost $\mathrm{c}$ has the form (4.37), then (4.39) holds: It is sufficient to approximate $F_{i}$ by an increasing and pointwise converging sequence $F_{i}^{n} \in \Gamma\left(\mathbb{R}_{+}\right)$; we will denote by $\mathrm{ET}^{n}$ the corresponding optimal Entropy-Transport functional. The corresponding sequence $\left(F_{i}^{n}\right)^{\circ}: \varphi_{i} \mapsto$ $\sup _{s>0}\left(F_{i}^{n}(s)+s \varphi_{i}\right)$ of conjugate concave functions is also nondecreasing and pointwise converging to $F_{i}^{\circ}$. By the previous step, if $E_{n}<\mathrm{ET}^{n}\left(\mu_{1}, \mu_{2}\right)$ with $\lim _{n \rightarrow \infty} E_{n}=\lim _{n \rightarrow \infty} \mathrm{ET}^{n}\left(\mu_{1}, \mu_{2}\right)=\mathrm{ET}\left(\mu_{1}, \mu_{2}\right)$ (the latter limit follows by Lemma 3.9) we can find $\boldsymbol{\varphi}_{n} \in \boldsymbol{\Phi}$ such that

$$
E_{n} \leq \sum_{i} \int_{X_{i}}\left(F_{i}^{n}\right)^{\circ}\left(\varphi_{i}^{n}\right) \mathrm{d} \mu_{i} \leq \sum_{i} \int_{X_{i}} F_{i}^{\circ}\left(\varphi_{i}^{n}\right) \mathrm{d} \mu_{i}=\mathscr{D}\left(\varphi_{n} \mid \mu_{1}, \mu_{2}\right) .
$$

Passing to the limit $n \rightarrow \infty$ we conclude $\mathrm{ET}\left(\mu_{1}, \mu_{2}\right) \leq \mathrm{D}\left(\mu_{1}, \mu_{2}\right)$ as desired.

Step 5, conclusion. We show that (4.39) holds for a general cost $\mathrm{c}$ : Let C : $\boldsymbol{X} \rightarrow[0, \infty]$ be an arbitrary proper 1.s.c. cost and let us denote by $\left(\mathrm{C}^{\alpha}\right)_{\alpha \in \mathbb{A}}$ the class of costs characterized by (4.37) and majorized by $\mathrm{C}$. Then, $\mathbb{A}$ is a directed set with the pointwise order $\leq$, since maxima of a finite number of cost functions in $\mathbb{A}$ can still be expressed as in (4.37). It is not 
difficult to check that $\mathrm{C}=\sup _{\alpha \in \mathbb{A}} \mathrm{C}^{\alpha}=\lim _{\alpha \in \mathbb{A}} \mathrm{C}^{\alpha}$ so that by Lemma 3.9 $\operatorname{ET}\left(\mu_{1}, \mu_{2}\right)=\lim _{\alpha \in \mathbb{A}} \operatorname{ET}^{\alpha}\left(\mu_{1}, \mu_{2}\right)=\sup _{\alpha \in \mathbb{A}} \operatorname{ET}^{\alpha}\left(\mu_{1}, \mu_{2}\right)$, where $\mathbb{E T}^{\alpha}$ denotes the Entropy-Transport functional associated to $\mathrm{C}^{\alpha}$.

Thus for every $E<\mathrm{ET}\left(\mu_{1}, \mu_{2}\right)$ we can find $\alpha \in \mathbb{A}$ such that $\mathrm{ET}^{\alpha}\left(\mu_{1}, \mu_{2}\right)>$ $E$ and therefore, by the previous step, a pair $\varphi^{\alpha} \in \operatorname{LSC}_{s}\left(X_{1}, \stackrel{\circ}{\mathrm{D}}\left(F_{1}^{\circ}\right)\right) \times$ $\operatorname{LSC}_{s}\left(X_{2}, \stackrel{\circ}{\mathrm{D}}\left(F_{2}^{\circ}\right)\right)$ with such that $\varphi_{1}^{\alpha} \oplus \varphi_{2}^{\alpha} \leq \mathrm{c}^{\alpha}$ in $\boldsymbol{X}$ and $\mathscr{D}\left(\varphi^{\alpha} \mid \mu_{1}, \mu_{2}\right) \geq E$. Since $\mathrm{C}^{\alpha} \leq \mathrm{C}$ we have $\varphi^{\alpha} \in \boldsymbol{\Phi}$ and $\mathrm{ET}\left(\mu_{1}, \mu_{2}\right) \leq \mathrm{D}\left(\mu_{1}, \mu_{2}\right)$ follows.

Arguing as in Remark 2.8 we can change the spaces of test potentials $\varphi=$ $\left(\varphi_{1}, \varphi_{2}\right) \in \boldsymbol{\Phi}$ introduced in (4.11).

Corollary 4.12 The duality formula (4.39) [and the equivalence with (4.8)] still holds if we replace the spaces of simple lower semicontinuous functions $\operatorname{LSC}_{s}\left(X_{i}, \stackrel{\circ}{\mathrm{D}}\left(F_{i}^{\circ}\right)\right)$ (resp. $\operatorname{LSC}_{s}\left(X_{i}, \stackrel{\circ}{\mathrm{D}}\left(R_{i}^{*}\right)\right)$ ) in the definition of $\boldsymbol{\Phi}($ resp. $\boldsymbol{\Psi})$ with the corresponding spaces of bounded lower semicontinuous functions $\mathrm{LSC}_{b}$ or with the spaces of bounded Borel functions $\mathrm{B}_{b}$.

If $\left(X_{i}, \tau_{i}\right)$ are completely regular spaces, then we can equivalently replace lower semicontinuous functions by continuous ones, obtaining

$$
\begin{aligned}
\mathrm{ET}\left(\mu_{1}, \mu_{2}\right)= & \sup \left\{\sum_{i} \int_{X_{i}} F^{\circ}\left(\varphi_{i}\right) \mathrm{d} \mu_{i}: \varphi_{i}, F_{i}^{\circ}\left(\varphi_{i}\right) \in \mathrm{C}_{b}\left(X_{i}\right), \varphi_{1} \oplus \varphi_{2} \leq \mathrm{c}\right\} \\
= & \sup \left\{\sum_{i} \int_{X_{i}} \psi_{i} \mathrm{~d} \mu_{i}: \psi_{i}, R_{i}^{*}\left(\psi_{i}\right) \in \mathrm{C}_{b}\left(X_{i}\right),\right. \\
& \left.R_{1}^{*}\left(\psi_{1}\right) \oplus R_{2}^{*}\left(\psi_{2}\right) \leq \mathrm{c}\right\} .
\end{aligned}
$$

Corollary 4.13 (Subadditivity of ET) The functional ET is convex and positively 1-homogeneous. In particular it is subadditive, in the sense that for every $\mu_{i}, \mu_{i}^{\prime} \in \mathcal{M}(X)$ and $\lambda \geq 0$ we have

$$
\begin{aligned}
\operatorname{ET}\left(\lambda \mu_{1}, \lambda \mu_{2}\right) & =\lambda \operatorname{ET}\left(\mu_{1}, \mu_{2}\right), \\
\operatorname{ET}\left(\mu_{1}+\mu_{1}^{\prime}, \mu_{2}+\mu_{2}^{\prime}\right) & \leq \mathrm{ET}\left(\mu_{1}, \mu_{2}\right)+\operatorname{ET}\left(\mu_{1}^{\prime}, \mu_{2}^{\prime}\right) .
\end{aligned}
$$

Proof By Theorem 4.11 it is sufficient to prove the corresponding property of $D$, which follows immediately from its representation formula (4.8) as a supremum of linear functionals.

\subsection{Existence of optimal Entropy-Kantorovich potentials}

In this section we will consider two cases, when the dual problem admits a pair of optimal Entropy-Kantorovich potentials $\boldsymbol{\varphi}=\left(\varphi_{1}, \varphi_{2}\right)$.

The first case is completely analogous to the transport setting. 
Theorem 4.14 In the basic coercive setting of Sect. 3.1 (i.e. (3.2a) or (3.2b) hold) let us suppose that $\left(X_{i}, \mathrm{~d}_{i}\right), i=1,2$, are complete metric spaces, that (3.12) holds, and that $\mathrm{c}$ is bounded and uniformly continuous with respect to the product distance $\mathrm{d}\left(\left(x_{1}, x_{2}\right),\left(x_{1}^{\prime} x_{2}^{\prime}\right)\right):=\sum_{i} \mathrm{~d}_{i}\left(x_{i}, x_{i}^{\prime}\right)$ in $\boldsymbol{X}=X_{1} \times X_{2}$. Then there exists a pair of optimal Entropy-Kantorovich potentials $\varphi \in \mathrm{C}_{b}\left(X_{1}\right) \times$ $\mathrm{C}_{b}\left(X_{2}\right)$ satisfying

$$
\varphi_{1} \oplus \varphi_{2} \leq \mathrm{C}, \quad \varphi_{i} \geq-\left(F_{i}\right)_{\infty}^{\prime}, \quad \mathrm{ET}\left(\mu_{1}, \mu_{2}\right)=\mathscr{D}\left(\varphi \mid \mu_{1}, \mu_{2}\right)
$$

Proof By the boundedness and uniform continuity of $\mathrm{c}$ we can find a continuous and concave modulus of continuity $\omega:[0, \infty) \rightarrow[0, \infty)$ with $\omega(0)=0$ such that

$$
\begin{aligned}
& \left|\mathrm{c}\left(x_{1}^{\prime}, x_{2}\right)-\mathrm{c}\left(x_{1}, x_{2}\right)\right| \leq \omega\left(\mathrm{d}_{1}\left(x_{1}^{\prime}, x_{1}\right)\right), \\
& \left|\mathrm{c}\left(x_{1}, x_{2}^{\prime}\right)-\mathrm{c}\left(x_{1}, x_{2}\right)\right| \leq \omega\left(\mathrm{d}_{2}\left(x_{2}^{\prime}, x_{2}\right)\right) .
\end{aligned}
$$

Possibly replacing the distances $d_{i}$ with $d_{i}+\omega\left(d_{i}\right)$, we may assume that $x_{1} \mapsto \mathrm{c}\left(x_{1}, x_{2}\right)$ is 1-Lipschitz w.r.t. $\mathrm{d}_{1}$ for every $x_{2} \in X_{2}$ and $x_{2} \mapsto \mathrm{c}\left(x_{1}, x_{2}\right)$ is 1-Lipschitz with respect to $\mathrm{d}_{2}$ for every $x_{1} \in X_{1}$. In particular, every ctransform (4.34) of a bounded function is 1-Lipschitz (and in particular Borel).

We apply Corollary 4.12: let $\boldsymbol{\varphi}_{n}$ be a maximizing sequence in $\boldsymbol{\Phi}$. By Lemma 4.9 we can assume that $\varphi_{n}$ is uniformly bounded; by (4.35) and (4.36) we can also assume that $\varphi_{n}$ are C-concave and thus 1-Lipschitz. If $K_{i, n}$ is a family of compact sets whose union $A_{i}$ has a full $\mu_{i}$ measure in $X_{i}$, by applying the Ascoli-Arzelà theorem on each compact set $K_{i, n}$ and a standard diagonal argument, we can extract a subsequence (still denoted by $\varphi_{n}$ ) pointwise convergent to $\varphi=\left(\varphi_{1}, \varphi_{2}\right)$ in $A_{1} \times A_{2}$. By setting $\varphi_{i}:=\liminf _{n \rightarrow \infty} \varphi_{i, n}, i=1$, 2, we extend $\boldsymbol{\varphi}$ to $\boldsymbol{X}$ and we obtain a pair $\varphi_{i} \in \mathrm{B}_{b}\left(X_{i}\right)$ satisfying $\varphi_{1} \oplus \varphi_{2} \leq \mathrm{C}$, $\varphi_{i} \geq-\left(F_{i}\right)_{\infty}^{\prime}$ and

$$
\begin{aligned}
\mathscr{D}\left(\varphi \mid \mu_{1}, \mu_{2}\right) & =\sum_{i} \int_{A_{i}} F_{i}^{\circ}\left(\varphi_{i}\right) \mathrm{d} \mu_{i} \\
& \geq \lim _{n \rightarrow \infty} \sum_{i} \int_{A_{i}} F_{i}^{\circ}\left(\varphi_{i, n}\right) \mathrm{d} \mu_{i}=\mathrm{ET}\left(\mu_{1}, \mu_{2}\right),
\end{aligned}
$$

thanks to the pointwise convergence in $A_{i}$, Fatou's Lemma and the fact that $F_{i}^{\circ}\left(\varphi_{i, n}\right)$ are uniformly bounded from above since $\varphi_{i, n}$ are uniformly bounded. Eventually replacing $\left(\varphi_{1}, \varphi_{2}\right)$ with $\left(\varphi_{1}^{\mathrm{CC}}, \varphi_{1}^{\mathrm{C}}\right)$ we obtain a pair in $\mathrm{C}_{b}\left(X_{1}\right) \times$ $\mathrm{C}_{b}\left(X_{2}\right)$ satisfying (4.42) thanks to Proposition 4.4.

The next result is of different type, since it does not require any boundedness nor regularity of $\mathrm{c}$ (also allowing the value $+\infty$ if $F_{i}(0)<\infty$ ). 
Theorem 4.15 In the basic coercive setting of Sect. 3.1 (i.e. (3.2a) or (3.2b) hold), let $\mu_{i} \in \mathcal{M}\left(X_{i}\right)$ with $\mathrm{ET}\left(\mu_{1}, \mu_{2}\right)<\infty$ (i.e. Problem 3.1 is feasible) and let us suppose that at least one of the following two conditions hold:

(a) $\mathrm{C}$ is everywhere finite and (3.12) holds

(b) $F_{i}(0)<\infty$.

Then a plan $\boldsymbol{\gamma} \in \mathcal{M}(\boldsymbol{X})$ is optimal if and only if there exists a pair $\boldsymbol{\varphi}$ as in (4.22) satisfying the optimality conditions (4.21) with respect to a Borel partition $\left(A_{i}, A_{\mu_{i}}, A_{\gamma_{i}}\right)$ related to the Lebesgue decomposition of $\left(\gamma_{i}, \mu_{i}\right)$ as in Lemma 2.3 .

Our proof starts with an auxiliary result on subdifferentials, which will be used extensively.

Lemma 4.16 Let $F \in \Gamma\left(\mathbb{R}_{+}\right)$, $s \in \mathrm{D}(F)$, let $\phi \in \mathbb{R} \cup\{ \pm \infty\}$ be an accumulation point of a sequence $\left(\phi_{n}\right) \subset \mathbb{R}$ satisfying

$$
\lim _{n \rightarrow \infty}\left(F(s)-s \phi_{n}+F^{*}\left(\phi_{n}\right)\right)=0 .
$$

If $\phi \in \mathbb{R}$ then $\phi \in \partial F(s)$, if $\phi=+\infty$ then $s=\max \mathrm{D}(F)$ and if $\phi=-\infty$ then $s=\min \mathrm{D}(F)$. In particular, if $s \in \mathrm{D}(F)$ then $\phi$ is finite.

Proof Up to extracting a suitable subsequence, it is not restrictive to assume that $\phi$ is the limit of $\phi_{n}$ as $n \rightarrow \infty$. For every $w \in \mathrm{D}(F)$ the Young inequality $w \phi_{n} \leq F(w)+F^{*}\left(\phi_{n}\right)$ yields

$$
\begin{aligned}
\limsup _{n \rightarrow \infty}(w-s) \phi_{n} & \leq \limsup _{n \rightarrow \infty} F(w)-F(s)+\left(F(s)-s \phi_{n}+F^{*}\left(\phi_{n}\right)\right) \\
= & F(w)-F(s)
\end{aligned}
$$

If $\mathrm{D}(F)=\{s\}$ then $\partial F(s)=\mathbb{R}$ and there is nothing to prove; let us assume that $\mathrm{D}(F)$ has nonempty interior.

If $\phi \in \mathbb{R}$ then $(w-s) \phi \leq F(w)-F(s)$ for every $w \in \mathrm{D}(F)$, so that $\phi \in \partial F(s)$. Since the right-hand side of (4.44) is finite for every $w \in \mathrm{D}(F)$, if $\phi=+\infty$ then $w \leq s$ for every $w \in \mathrm{D}(F)$, so that $s=\max \mathrm{D}(F)$. An analogous argument holds when $\phi=-\infty$.

Proof of Theorem 4.15 We already proved (Theorem 4.6) that the existence of a pair $\boldsymbol{\varphi}$ as in (4.22) satisfying (4.21) yields the optimality of $\boldsymbol{\gamma}$.

Let us now assume that $\boldsymbol{\gamma} \in \mathcal{M}(\boldsymbol{X})$ is optimal. If $\mu_{i} \equiv \eta_{0}$, then we also have $\boldsymbol{\gamma}=0$ and (4.21) is always satisfied, since we can choose $\varphi_{i} \equiv 0$.

We can therefore assume that at least one of the measures $\mu_{i}$, say $\mu_{2}$, has positive mass. Let $\gamma \in \operatorname{Opt}_{E T}\left(\mu_{1}, \mu_{2}\right)$, and let us apply Theorem 4.11 to find a maximizing sequence $\boldsymbol{\varphi}_{n} \in \boldsymbol{\Phi}$ such that $\lim _{n \uparrow \infty} \mathscr{D}\left(\boldsymbol{\varphi}_{n} \mid \mu_{1}, \mu_{2}\right)=$ 
$\mathrm{ET}\left(\mu_{1}, \mu_{2}\right)$. Using the Borel partitions $\left(A_{i}, A_{\mu_{i}}, A_{\gamma_{i}}\right)$ for the pairs of measures $\gamma_{i}, \mu_{i}$ provided by Lemma 2.3 and observing that the vanishing difference $\mathrm{ET}\left(\mu_{1}, \mu_{2}\right)-\mathscr{D}\left(\varphi_{n} \mid \mu_{1}, \mu_{2}\right)$ can be decomposed in the sum of the following three nonnegative contributions

$$
\begin{aligned}
\mathscr{E}\left(\boldsymbol{\gamma} \mid \mu_{1}, \mu_{2}\right)-\mathscr{D}\left(\boldsymbol{\varphi}_{n} \mid \mu_{1}, \mu_{2}\right)= & \int_{X_{1} \times X_{2}}\left(\mathrm{c}\left(x_{1}, x_{2}\right)-\varphi_{1, n}\left(x_{1}\right)-\varphi_{2, n}\left(x_{2}\right)\right) \mathrm{d} \boldsymbol{\gamma} \\
& +\int_{A_{i} \cup A_{\mu_{i}}}\left(F_{i}\left(\sigma_{i}\right)+\sigma_{i} \varphi_{i, n}-F_{i}^{\circ}\left(\varphi_{i, n}\right)\right) \mathrm{d} \mu_{i} \\
& +\int_{A_{\gamma_{i}}}\left(\varphi_{i, n}+\left(F_{i}\right)_{\infty}^{\prime}\right) \mathrm{d} \gamma_{i}^{\perp}
\end{aligned}
$$

we get

$$
\begin{array}{r}
\lim _{n \rightarrow \infty} \int_{X_{1} \times X_{2}}\left(\mathrm{c}\left(x_{1}, x_{2}\right)-\varphi_{1, n}\left(x_{1}\right)-\varphi_{2, n}\left(x_{2}\right)\right) \mathrm{d} \gamma=0, \\
\lim _{n \rightarrow \infty} \int_{A_{i} \cup A_{\mu_{i}}}\left(F_{i}\left(\sigma_{i}\right)+\sigma_{i} \varphi_{i, n}-F_{i}^{\circ}\left(\varphi_{i, n}\right)\right) \mathrm{d} \mu_{i}=0, \\
\lim _{n \rightarrow \infty} \int_{A_{\gamma_{i}}}\left(\varphi_{i, n}+\left(F_{i}\right)_{\infty}^{\prime}\right) \mathrm{d} \gamma_{i}^{\perp}=0 .
\end{array}
$$

Since all the integrands are nonnegative, up to selecting a suitable subsequence (not relabeled) we can assume that the integrands are converging pointwise a.e. to 0 . We can thus find Borel sets $A_{i}^{\prime} \subset A_{i}, A_{\mu_{i}}^{\prime} \subset A_{\mu_{i}}, A_{\gamma_{i}}^{\prime} \subset A_{\gamma_{i}}$ and $A^{\prime} \subset \boldsymbol{X}$ with $\pi^{i}\left(A^{\prime}\right)=A_{i}^{\prime} \cup A_{\gamma_{i}}^{\prime},\left(\mu_{i}+\gamma_{i}\right)\left(\left(A_{i} \backslash A_{i}^{\prime}\right) \cup\left(A_{\mu_{i}} \backslash A_{\mu_{i}}^{\prime}\right) \cup\left(A_{\gamma_{i}} \backslash A_{\gamma_{i}}^{\prime}\right)\right)=0$, and $\boldsymbol{\gamma}\left(\boldsymbol{X} \backslash A^{\prime}\right)=0$ such that

$$
\begin{aligned}
\mathrm{c}\left(x_{1}, x_{2}\right)<\infty, \quad \lim _{n \rightarrow \infty} \mathrm{c}\left(x_{1}, x_{2}\right)-\varphi_{1, n}\left(x_{1}\right)-\varphi_{2, n}\left(x_{2}\right)=0 & \text { in } A^{\prime}, \\
F_{i}\left(\sigma_{i}\right)<\infty, \quad \lim _{n \rightarrow \infty} F_{i}\left(\sigma_{i}\right)+\sigma_{i} \varphi_{i, n}-F_{i}^{\circ}\left(\varphi_{i, n}\right)=0 & \text { in } A_{i}^{\prime} \cup A_{\mu_{i}}^{\prime}, \\
\lim _{n \rightarrow \infty}\left(\varphi_{i, n}+\left(F_{i}\right)_{\infty}^{\prime}\right)=0 & \text { in } A_{\gamma_{i}}^{\prime} .
\end{aligned}
$$

For every $x_{i} \in X_{i}$ we define the Borel functions $\varphi_{1}\left(x_{1}\right):=\lim _{\sup _{n \rightarrow \infty}} \varphi_{1, n}\left(x_{1}\right)$ and $\varphi_{2}\left(x_{2}\right):=\liminf _{n \rightarrow \infty} \varphi_{2, n}\left(x_{2}\right)$, taking values in $\mathbb{R} \cup\{ \pm \infty\}$. It is clear that the pair $\boldsymbol{\varphi}=\left(\varphi_{1}, \varphi_{2}\right)$ complies with (4.22), (4.21d) and (4.21c).

If $\boldsymbol{\gamma}(\boldsymbol{X})=0$ then (4.21a) and (4.21b) are trivially satisfied, so that it is not restrictive to assume $\boldsymbol{\gamma}(\boldsymbol{X})>0$. 
If $\mu_{1}\left(X_{1}\right)=0$ then $\left(F_{1}\right)_{\infty}^{\prime}$ is finite (since $\gamma_{1}^{\perp}\left(X_{1}\right)=\gamma_{1}\left(X_{1}\right)=\boldsymbol{\gamma}(\boldsymbol{X})>0$ ) and $\varphi_{1} \equiv\left(F_{1}\right)_{\infty}^{\prime}$ on $A_{\gamma_{1}}^{\prime}$ and on $A^{\prime}$. It follows that $\varphi_{2}\left(x_{2}\right)=\mathrm{c}\left(x_{1}, x_{2}\right)-$ $\left(F_{1}\right)_{\infty}^{\prime} \in \mathbb{R}$ on $A^{\prime}$ so that (4.21a) is satisfied. Since $\varphi_{2}\left(x_{2}\right)$ is an accumulation point of $\varphi_{2, n}\left(x_{2}\right)$, Lemma 4.16 yields $-\varphi_{2}\left(x_{2}\right) \in \partial F_{2}\left(\sigma_{2}\left(x_{2}\right)\right)$ in $A_{2}^{\prime}$ so that (4.21b) is also satisfied (in the case $i=1$ one can choose $A_{1}^{\prime}=\emptyset$ ).

We can thus assume that $\mu_{i}\left(X_{i}\right)>0$ and $\boldsymbol{\gamma}(\boldsymbol{X})>0$. In order to check (4.21a) and (4.21b) we distinguish two cases.

Case a: $\mathrm{C}$ is everywhere finite and (3.12) holds. Let us first prove that $\varphi_{1}<\infty$ everywhere.

By contradiction, if there is a point $\bar{x}_{1} \in X_{1}$ such that $\varphi_{1}\left(\bar{x}_{1}\right)=\infty$ we deduce that $\varphi_{2}\left(x_{2}\right)=-\infty$ for every $x_{2} \in X_{2}$.

Since the set $A_{2}^{\prime} \cup A_{\mu_{2}}^{\prime}$ has positive $\mu_{2}$-measure, it contains some point $\bar{x}_{2}$ : Equation (4.46) and Lemma 4.16 (with $F=F_{2}, s=\sigma_{2}\left(\bar{x}_{2}\right), \phi_{n}:=$ $\left.-\varphi_{2, n}\left(\bar{x}_{2}\right)\right)$ yield $s_{2}^{+}=\max \mathrm{D}\left(F_{2}\right)=\sigma_{2}\left(\bar{x}_{2}\right)<\infty$ and $\sigma_{2} \equiv s_{2}^{+}$in $A_{2}^{\prime} \cup A_{\mu_{2}}^{\prime}$. We thus have $\mathrm{D}\left(F_{2}\right) \subset\left[0, s_{2}^{+}\right],\left(F_{2}\right)_{\infty}^{\prime}=+\infty$ and therefore $m_{2} s_{2}^{+}=\boldsymbol{\gamma}(\boldsymbol{X})$.

On the other hand, if $\varphi_{2}=-\infty$ in $X_{2}$ we deduce that $\varphi_{1}\left(x_{1}\right)=+\infty$ for every $x_{1} \in \pi^{1}\left(A^{\prime}\right)$. Since $\left(F_{1}\right)_{\infty}^{\prime} \geq 0$, it follows that $\gamma_{i}\left(A_{\gamma_{i}}^{\prime}\right)=0$ (i.e. $\gamma_{i}^{\perp}=0$ ) so that there is a point $a_{1}$ in $A_{1}^{\prime}$ such that $\varphi_{1}\left(a_{1}\right)=+\infty$. Arguing as before, a further application of Lemma 4.16 yields that $\sigma_{1} \equiv s_{1}^{-}=\min \mathrm{D}\left(F_{1}\right) \mu_{1}$-a.e. It follows that $m_{1} s_{1}^{-}=\gamma_{1}\left(X_{1}\right)=\boldsymbol{\gamma}(\boldsymbol{X})=m_{2} s_{2}^{+}$, and this contradicts (3.12).

Since $\mu_{1}\left(X_{1}\right)>0$ the same argument shows that $\varphi_{2}<\infty$ everywhere in $X_{2}$. It follows that (4.21a) holds and $\varphi_{i}>-\infty$ on $A_{i}^{\prime}$. Since $\varphi_{i}\left(x_{i}\right)$ is an accumulation point of $\varphi_{i, n}\left(x_{i}\right)$, Lemma 4.16 yields $-\varphi_{i}\left(x_{i}\right) \in \partial F_{i}\left(\sigma_{i}\left(x_{i}\right)\right)$ in $A_{i}^{\prime}$ so that $(4.21 \mathrm{~b})$ is also satisfied.

Case b: $F_{i}(0)<\infty$. In this case $F_{i}^{\circ}$ are bounded from above and $\varphi_{i} \geq$ $-\left(F_{i}\right)_{\infty}^{\prime}$ everywhere in $X_{i}$. By Theorem $4.11 \lim _{n \rightarrow \infty} \sum_{i} \int F_{i}^{\circ}\left(\varphi_{i, n}\right) \mathrm{d} \mu_{i}>$ $-\infty$, so that Fatou's Lemma yields $F_{1}^{\circ}\left(\varphi_{1}\right) \in \mathrm{L}^{1}\left(X_{1}, \mu_{1}\right)$ and $\varphi_{1}\left(x_{1}\right)>-\infty$ for $\mu_{1}$-a.e. $x_{1} \in X_{1}$, in particular for $\left(\mu_{1}+\gamma_{1}\right)$-a.e. $x_{1} \in A_{1}^{\prime}$. Applying Lemma 4.16, since $\sigma_{1}\left(x_{1}\right)>0=\min \mathrm{D}\left(F_{1}\right)$ in $A_{1}^{\prime}$, we deduce that $-\varphi_{1}\left(x_{1}\right) \in$ $\partial F_{1}\left(\sigma_{1}\left(x_{1}\right)\right)$ for $\left(\mu_{1}+\gamma_{1}\right)$-a.e. $x_{1} \in A_{1}^{\prime}$, i.e. (4.21b) for $i=1$. Since we already checked that (4.21c) and (4.21d) hold, applying Lemma 2.6 (with $\phi:=-\varphi_{1}$ and $\left.\left.\psi:=F_{1}^{\circ}\left(\varphi_{1}\right)\right)\right)$ we get $\varphi_{1} \in \mathrm{L}^{1}\left(X_{1}, \gamma_{1}\right)$, in particular $\varphi_{1} \circ \pi^{1} \in \mathbb{R}$ holds $\boldsymbol{\gamma}$-a.e. in $\boldsymbol{X}$. It follows that (4.21a) holds and $\varphi_{2} \circ \pi^{2} \in \mathrm{L}^{1}(\boldsymbol{X}, \boldsymbol{\gamma})$ so that $\varphi_{2} \in \mathbb{R}$ $\left(\mu_{2}+\gamma_{2}\right)$-a.e. in $A_{2}^{\prime}$. A further application of Lemma 4.16 yields (4.21b) for $i=2$.

Corollary 4.17 Let us suppose that $\mathrm{D}\left(F_{i}\right) \supset(0, \infty)$ and $F_{i}$ are differentiable in $(0, \infty)$ and let $\mu_{i} \in \mathcal{M}\left(X_{i}\right)$ with $\mathrm{ET}\left(\mu_{1}, \mu_{2}\right)<\infty$. A plan $\gamma \in \mathcal{M}(\boldsymbol{X})$ belongs to $\operatorname{Opt}_{\mathrm{ET}}\left(\mu_{1}, \mu_{2}\right)$ if and only if there exist Borel partitions $\left(A_{i}, A_{\mu_{i}}, A_{\gamma_{i}}\right)$ and corresponding Borel densities $\sigma_{i}$ associated to $\gamma_{i}$ and $\mu_{i}$ as in Lemma 2.3 such that setting 


$$
\varphi_{i}\left(x_{i}\right):= \begin{cases}-F_{i}^{\prime}\left(\sigma_{i}\right) & \text { if } x_{i} \in A_{i} \\ -\left(F_{i}\right)_{0}^{\prime} & \text { if } x_{i} \in A_{\mu_{i}}, \\ -\left(F_{i}\right)_{\infty}^{\prime} & \text { if } x_{i} \in X_{i} \backslash\left(A_{i} \cup A_{\mu_{i}}\right),\end{cases}
$$

we have

$$
\begin{aligned}
\varphi_{1} \oplus_{o} \varphi_{2} & \leq \mathrm{c} \text { in } X_{1} \times X_{2}, \\
\varphi_{1} \oplus \varphi_{2} & =\mathrm{c} \boldsymbol{\gamma} \text {-a.e. in }\left(A_{1} \cup A_{\gamma_{1}}\right) \times\left(A_{2} \cup A_{\gamma_{2}}\right) .
\end{aligned}
$$

Proof Since $\partial F_{i}(s)=\left\{F_{i}^{\prime}(s)\right\}$ for every $s \in(0, \infty)$ and $F_{i}^{\circ}\left(\varphi_{i}\right)=F_{i}(0)$ if and only if $\varphi_{i} \in\left[-\left(F_{i}\right)_{0}^{\prime}, \infty\right],(4.49)$ is clearly a necessary condition for optimality, thanks to Theorem 4.15. Since $\left(F_{i}\right)_{0}^{\prime} \leq F_{i}^{\prime}(s) \leq\left(F_{i}\right)_{\infty}^{\prime}$ Theorem 4.6 shows that conditions (4.48)-(4.49) are also sufficient.

The next result (where we will keep the same notation of Corollary 4.17) shows that (4.48)-(4.49) take an even simpler form when $-\left(F_{i}\right)_{0}^{\prime}=$ $\left(F_{i}\right)_{\infty}^{\prime}=+\infty$; in particular, by assuming that $\mathrm{c}$ is continuous, the support of the marginals $\gamma_{i}$ of an optimal plan $\gamma$ cannot be too small, since $\operatorname{supp}\left(\gamma_{i}\right) \supset \operatorname{supp}\left(\mu_{i}\right) \backslash \operatorname{supp}\left(\mu_{i}^{\perp}\right)$.

Corollary 4.18 (Spread of the support) Let us suppose that

- $\mathrm{C}: \boldsymbol{X} \rightarrow[0, \infty]$ is continuous.

- $\mathrm{D}\left(F_{i}\right) \supset(0, \infty), F_{i}$ are differentiable in $(0, \infty)$, and $-\left(F_{i}\right)_{0}^{\prime}=\left(F_{i}\right)_{\infty}^{\prime}=$ $\infty$,

and let $\mu_{i} \in \mathcal{M}\left(X_{i}\right)$ with $\mathrm{ET}\left(\mu_{1}, \mu_{2}\right)<\infty$ and $\boldsymbol{\gamma} \in \mathcal{M}(\boldsymbol{X})$. Then $\boldsymbol{\gamma}$ is an optimal plan if and only if $\gamma_{i} \ll \mu_{i}$, for every $\left(x_{1}, x_{2}\right) \in \operatorname{supp}\left(\mu_{1}\right) \times \operatorname{supp}\left(\mu_{2}\right)$ we have $\mathrm{c}\left(x_{1}, x_{2}\right)=+\infty$ if $x_{1} \in \operatorname{supp} \mu_{1}^{\perp}$ or $x_{2} \in \operatorname{supp} \mu_{2}^{\perp}$, and there exist Borel sets $A_{i} \subset \operatorname{supp} \gamma_{i}$ with $\gamma_{i}\left(X_{i} \backslash A_{i}\right)=0$ and Borel densities $\sigma_{i}: A_{i} \rightarrow(0, \infty)$ of $\gamma_{i}$ w.r.t. $\mu_{i}$ such that

$$
\begin{aligned}
& F_{1}^{\prime}\left(\sigma_{1}\right) \oplus F_{2}^{\prime}\left(\sigma_{2}\right) \geq-\mathrm{c} \text { in } A_{1} \times A_{2}, \\
& F_{1}^{\prime}\left(\sigma_{1}\right) \oplus F_{2}^{\prime}\left(\sigma_{2}\right)=-\mathrm{c} \quad \gamma \text {-a.e. in } A_{1} \times A_{2} .
\end{aligned}
$$

Remark 4.19 Apart from the case of pure transport problems (Example E.3 of Sect. 3.3), where the existence of Kantorovich potentials is well known (see [50, Thm. 5.10]), Theorem 4.15 covers essentially all the interesting cases, at least when the cost $c$ takes finite values if $0 \notin \mathrm{D}\left(F_{i}\right)$. In fact, if the strengthened feasibility condition (3.12) does not hold, it is not difficult to construct an example of optimal plan $\gamma$ for which conditions (4.22), (4.21a), (4.21b) cannot be satisfied. Consider e.g. $X_{i}=\mathbb{R}, \mathrm{c}\left(x_{1}, x_{2}\right):=\frac{1}{2}\left|x_{1}-x_{2}\right|^{2}$, $\mu_{1}:=\mathrm{e}^{-\sqrt{\pi} x_{1}^{2}} \mathscr{L}^{1}, \mu_{2}:=\mathrm{e}^{-\sqrt{\pi}\left(x_{2}+1\right)^{2}} \mathscr{L}^{1}$, and entropy functions $F_{i}$ satisfying $\mathrm{D}\left(F_{1}\right)=[a, 1], \mathrm{D}\left(F_{2}\right)=[1, b]$ with arbitrary choice of $a \in[0,1)$ 
and $b \in(1, \infty]$. Since $m_{1}=m_{2}=1$ the weak feasibility condition (3.1) holds, but (3.12) is violated. We find $\gamma_{i}=\mu_{i}, \sigma_{i} \equiv 1$, so that the optimal plan $\gamma$ can be obtained by solving the quadratic optimal transportation problem, thus $\boldsymbol{\gamma}:=\boldsymbol{t}_{\sharp} \mu_{1}$ where $\boldsymbol{t}(x):=(x, x-1)$. In this case the potentials $\varphi_{i}$ are uniquely determined up to an additive constant $a \in \mathbb{R}$ so that we have $\varphi_{1}\left(x_{1}\right)=x_{1}+a, \varphi_{2}\left(x_{2}\right)=-x_{2}-a-\frac{1}{2}$, and it is clear that condition $-\varphi_{i} \in \partial F_{i}(1)$ corresponding to (4.21b) cannot be satisfied, since $\partial F_{i}(1)$ are always proper subsets of $\mathbb{R}$. We can also construct entropies such that $\partial F_{i}(1)=\varnothing$ (e.g. $F_{1}(r)=(1-r) \log (1-r)+r$ with $\mathrm{D}\left(F_{1}\right)=[0,1]$, $F_{2}(r)=(r-1) \log (r-1)-r+2$ with $\left.\mathrm{D}\left(F_{2}\right)=[1, \infty)\right)$ so that $(4.21 \mathrm{~b})$ can never hold, independently of the cost $\mathrm{C}$.

\section{5 "Homogeneous" formulations of optimal Entropy-Transport problems}

Starting from the reverse formulation of the Entropy-Transport problem of Sect. 3.5 via the functional $\mathscr{R}$, see (3.28), in this section we will derive further equivalent representations of the ET functional, which will also reveal new interesting properties, in particular when we will apply these results to the logarithmic Hellinger-Kantorovich functional. The advantage of the reverse formulation is that it always admits a "1-homogeneous" representation, associated to a modified cost functional that can be explicitly computed in terms of $R_{i}$ and c.

We will always tacitly assume the coercive setting of Sect. 3.1, see (3.2).

\subsection{The homogeneous marginal perspective functional}

First of all we introduce the marginal perspective function $H_{c}$ depending on the parameter $c \geq \inf c$ (see [23, Chap. IV, Sect.2.2] for the definition and the basic properties of the perspective; we use the term "marginal perspective" since we are infimizing w.r.t. the perspective parameter):

Definition 5.1 (Marginal perspective function and cost) For $c \in[0, \infty)$, the marginal perspective function $H_{c}:[0, \infty) \times[0, \infty) \rightarrow[0, \infty]$ is defined as the lower semicontinuous envelope of

$$
\begin{aligned}
\tilde{H}_{c}\left(r_{1}, r_{2}\right) & :=\inf _{\theta>0} \theta\left(R_{1}\left(r_{1} / \theta\right)+R_{2}\left(r_{2} / \theta\right)+c\right) \\
& =\inf _{\theta>0} r_{1} F_{1}\left(\theta / r_{1}\right)+r_{2} F_{2}\left(\theta / r_{2}\right)+\theta c .
\end{aligned}
$$

For $c=\infty$ we set

$$
H_{\infty}\left(r_{1}, r_{2}\right):=F_{1}(0) r_{1}+F_{2}(0) r_{2}
$$


The induced marginal perspective cost is $H:\left(X_{1} \times \mathbb{R}_{+}\right) \times\left(X_{2} \times \mathbb{R}_{+}\right) \rightarrow$ $[0, \infty]$ with

$$
H\left(x_{1}, r_{1} ; x_{2}, r_{2}\right):=H_{\mathrm{C}\left(x_{1}, x_{2}\right)}\left(r_{1}, r_{2}\right), \quad \text { for } x_{i} \in X_{i} \text { and } r_{i} \geq 0 .
$$

The last formula (5.2) is justified by the property $F_{i}(0)=\left(R_{i}\right)_{\infty}^{\prime}$ and the fact that $H_{c}\left(r_{1}, r_{2}\right) \uparrow H_{\infty}\left(r_{1}, r_{2}\right)$ as $c \uparrow \infty$ for every $r_{1}, r_{2} \in[0, \infty)$; see also Lemma 5.3 below.

The marginal perspective cost (5.3) has an important interpretation in terms of the optimal Entropy Transport problem 3.1 between two Dirac masses: at least in the superlinear case (3.2a), it is easy to see that for every $x_{i} \in X$ and $r_{i}>0, i=1,2$, we have

$$
H\left(x_{1}, r_{1} ; x_{2}, r_{2}\right)=\mathrm{ET}\left(r_{1} \delta_{x_{1}}, r_{2} \delta_{x_{2}}\right), \quad \text { when }\left(F_{i}\right)_{\infty}^{\prime}=+\infty .
$$

It is in fact sufficient to minimize $\mathscr{E}\left(\boldsymbol{\gamma} \mid r_{1} \delta_{x_{1}}, r_{2} \delta_{x_{2}}\right)$ among the plans $\boldsymbol{\gamma}$ of the form $\theta \delta_{\left(x_{1}, x_{2}\right)}$.

Example 5.2 Let us consider the symmetric cases associated to the entropies $U_{p}$ introduced in (1.4) and Example 2.5 and $V(s)=|s-1|$ :

E.1 In the "logarithmic entropy case", which we will extensively study in Part II, we have

$$
F_{i}(s):=U_{1}(s)=s \log s-(s-1) \text { and } R_{i}(r)=U_{0}(r)=r-1-\log r .
$$

A direct computation shows

$$
\begin{aligned}
\tilde{H}_{c}\left(r_{1}, r_{2}\right) & =H_{c}\left(r_{1}, r_{2}\right)=r_{1}+r_{2}-2 \sqrt{r_{1} r_{2}} \mathrm{e}^{-c / 2} \\
& =\left(\sqrt{r_{1}}-\sqrt{r_{2}}\right)^{2}+2 \sqrt{r_{1} r_{2}}\left(1-\mathrm{e}^{-c / 2}\right) .
\end{aligned}
$$

E.2 For $p=0, F_{i}(s)=U_{0}(s)=s-\log s-1$, and $R_{i}(r)=U_{1}(r)$ we obtain

$$
\tilde{H}_{c}\left(r_{1}, r_{2}\right)=H_{c}\left(r_{1}, r_{2}\right)=r_{1} \log r_{1}+r_{2} \log r_{2}-\left(r_{1}+r_{2}\right) \log \left(\frac{r_{1}+r_{2}}{2+c}\right) .
$$

E.3 In the power-like case with $p \in \mathbb{R} \backslash\{0,1\}$ we start from

$$
F_{i}(s):=U_{p}(s)=\frac{1}{p(p-1)}\left(s^{p}-p(s-1)-1\right), \quad R_{i}(r)=U_{1-p}(r)
$$

and obtain, for $r_{1}, r_{2}>0$,

$$
\tilde{H}_{c}\left(r_{1}, r_{2}\right)=H_{c}\left(r_{1}, r_{2}\right)
$$




$$
=\frac{1}{p}\left[\left(r_{1}+r_{2}\right)-\frac{r_{1} r_{2}}{\left(r_{1}^{p-1}+r_{2}^{p-1}\right)^{1 /(p-1)}}(2-(p-1) c)_{+}^{q}\right] \text {, }
$$

where $q=p /(p-1)$. In fact, we have

$$
\begin{aligned}
& \theta\left(U_{1-p}\left(\frac{r_{1}}{\theta}\right)+U_{1-p}\left(\frac{r_{2}}{\theta}\right)+c\right)=\frac{r_{1}^{1-p}+r_{2}^{1-p}}{p(p-1)} \theta^{p} \\
&+\left.\frac{1}{p}\left(r_{1}+r_{2}\right)+\frac{1}{p-1}((p-1) c-2) \theta\right) \\
&=\frac{1}{p}\left(r_{1}+r_{2}\right)+\frac{1}{p-1}\left[\frac{1}{p}\left(\left(r_{1}^{1-p}+r_{2}^{1-p}\right)^{1 / p} \theta\right)^{p}-(2-(p-1) c) \theta\right],
\end{aligned}
$$

and (5.7) follows by minimizing w.r.t. $\theta$. For example, when $p=q=2$,

$$
\begin{aligned}
H_{c}\left(r_{1}, r_{2}\right) & =\frac{1}{2}\left(r_{1}+r_{2}\right)-\frac{1}{2} \frac{r_{1} r_{2}}{r_{1}+r_{2}}(2-c)_{+}^{2} \\
& =\frac{1}{2\left(r_{1}+r_{2}\right)}\left(\left(r_{1}-r_{2}\right)^{2}+h(c) r_{1} r_{2}\right),
\end{aligned}
$$

where $h(c)=c(4-c)$ if $0 \leq c \leq 2$ and 4 if $c \geq 2$. For $p=-1$ and $q=1 / 2$ equation (5.7) yields

$$
\tilde{H}_{c}\left(r_{1}, r_{2}\right)=H_{c}\left(r_{1}, r_{2}\right)=\sqrt{\left(r_{1}^{2}+r_{2}^{2}\right)(2+2 c)}-\left(r_{1}+r_{2}\right) .
$$

E.4 In the case of the total variation entropy $R_{i}(s)=V(s)=|s-1|$ we easily find

$$
\begin{aligned}
\tilde{H}_{c}\left(r_{1}, r_{2}\right) & =H_{c}\left(r_{1}, r_{2}\right)=r_{1}+r_{2}-(2-c)_{+}\left(r_{1} \wedge r_{2}\right) \\
& =\left|r_{2}-r_{1}\right|+(c \wedge 2)\left(r_{1} \wedge r_{2}\right) .
\end{aligned}
$$

The following dual characterization of $H_{c}$ nicely explains the crucial role of $H_{c}$.

Lemma 5.3 (Dual characterization of $H_{c}$ ) For every $c \geq 0$ the function $H_{c}$ admits the dual representation

$$
\begin{aligned}
& H_{c}\left(r_{1}, r_{2}\right) \\
& \quad=\sup \left\{r_{1} \psi_{1}+r_{2} \psi_{2}: \psi_{i} \in \mathrm{D}\left(R_{i}^{*}\right), R_{1}^{*}\left(\psi_{1}\right)+R_{2}^{*}\left(\psi_{2}\right) \leq c\right\} \\
& =\sup \left\{r_{1} F_{1}^{\circ}\left(\varphi_{1}\right)+r_{2} F_{2}^{\circ}\left(\varphi_{2}\right): \varphi_{i} \in \mathrm{D}\left(F_{i}^{\circ}\right), \varphi_{1}+\varphi_{2} \leq c\right\} .
\end{aligned}
$$


In particular it is lower semicontinuous, convex and positively 1-homogeneous (thus sublinear) with respect to $\left(r_{1}, r_{2}\right)$, nondecreasing and concave w.r.t. $c$, and satisfies

$$
H_{c}\left(r_{1}, r_{2}\right) \leq H_{\infty}\left(r_{1}, r_{2}\right)=\sum_{i} F_{i}(0) r_{i} \text { for every } c \geq 0, r_{i} \geq 0 .
$$

\section{Moreover,}

(a) the function $H_{c}$ coincides with $\tilde{H}_{c}$ in the interior of its domain; in particular, if $F_{i}(0)<\infty$ then $H_{c}\left(r_{1}, r_{2}\right)=\tilde{H}_{c}\left(r_{1}, r_{2}\right)$ whenever $r_{1} r_{2}>0$.

(b) If $\left(F_{1}\right)_{\infty}^{\prime}+c \geq-\left(F_{2}\right)_{0}^{\prime}$ and $\left(F_{2}\right)_{\infty}^{\prime}+c \geq-\left(F_{1}\right)_{0}^{\prime}$, then

$$
H_{c}\left(r_{1}, r_{2}\right)=\sum_{i} F_{i}(0) r_{i} \text { if } r_{1} r_{2}=0
$$

Proof Since $\sup \mathrm{D}\left(R_{i}^{*}\right)=F_{i}(0)$ by (2.33), one immediately gets (5.10) in the case $c=\infty$; we can thus assume $c<\infty$.

It is not difficult [23, Chap. IV, Sect.2.2] to check that the perspective function $\left(r_{1}, r_{2}, \theta\right) \mapsto \theta\left(R_{1}\left(r_{1} / \theta\right)+R_{2}\left(r_{2} / \theta\right)+c\right)$ is jointly convex in $[0, \infty) \times[0, \infty) \times(0, \infty)$ so that $\tilde{H}_{c}$ is a convex and positive 1 -homogeneous function. It is also proper (i.e. it is not identically $+\infty$ ) thanks to (3.1), and it is concave w.r.t $c$ since it is the infimum of a family of affine functions in $c$.

By Legendre duality [41, Thm.12.2], its lower semicontinuous envelope is given by

$$
H_{c}\left(r_{1}, r_{2}\right)=\sup \left\{\sum_{i} \psi_{i} r_{i}: H_{c}^{*}\left(\psi_{1}, \psi_{2}\right) \leq 0\right\}
$$

where

$$
\begin{aligned}
H_{c}^{*}\left(\psi_{1}, \psi_{2}\right) & =\sup \left\{\sum_{i} \psi_{i} r_{i}-\tilde{H}_{c}\left(r_{1}, r_{2}\right): r_{i} \geq 0\right\} \\
& =\sup _{r_{i} \geq 0, \theta>0} \sum_{i}\left(\psi_{i} r_{i}-\theta R_{i}\left(r_{i} / \theta\right)\right)-c \theta \\
& =\sup _{\theta>0} \theta\left(\sum_{i} R_{i}^{*}\left(\psi_{i}\right)-c\right) \\
& = \begin{cases}0 & \text { if } R_{i}^{*}\left(\psi_{i}\right)<\infty, \quad \sum_{i} R_{i}^{*}\left(\psi_{i}\right) \leq c \\
+\infty & \text { otherwise. }\end{cases}
\end{aligned}
$$

Now, (5.11) immediately follows from (5.10) by the usual change of variable $\varphi_{i}=R_{i}^{*}\left(\psi_{i}\right)$, recall (2.31) and $F_{i}^{\circ}\left(\varphi_{i}\right)=-F_{i}^{*}\left(-\varphi_{i}\right)$.

In order to prove point (a) it is sufficient to recall that convex functions are always continuous in the interior of their domain [41, Thm. 10.1]. In particular, 
since $\lim _{\theta \downarrow 0} \theta\left(R_{1}\left(r_{1} / \theta\right)+R_{2}\left(r_{2} / \theta\right)+c\right)=\sum_{i}\left(R_{i}\right)_{\infty}^{\prime} r_{i}=\sum_{i} F_{i}(0) r_{i}$ for every $r_{1}, r_{2}>0$, we have $\tilde{H}_{c}\left(r_{1}, r_{2}\right) \leq \sum_{i} F_{i}(0) r_{i}$, so that $\tilde{H}_{c}$ is always finite if $F_{i}(0)<\infty$.

Concerning (b), it is obvious when $r_{1}=r_{2}=0$. When $r_{1}>r_{2}=0$, the facts that $\sup \mathrm{D}\left(R_{i}^{*}\right)=F_{i}(0), \lim _{r \uparrow F_{i}(0)} R_{i}^{*}(r)=-\left(F_{i}\right)_{0}^{\prime}$, and inf $R_{i}^{*}=-\left(F_{i}\right)_{\infty}^{\prime}$ (see (2.33)) yield

$$
H_{c}\left(r_{1}, 0\right)=\sup \left\{\psi_{1} r_{1}: R_{1}^{*}\left(\psi_{1}\right) \leq c-\inf R_{2}^{*}\right\}=F_{1}(0) r_{1} .
$$

An analogous formula holds when $0=r_{1}<r_{2}$.

A simple consequence of Lemma 5.3 and (2.31) is the lower bound

$$
\tilde{H}_{c}\left(r_{1}, r_{2}\right) \geq H_{c}\left(r_{1}, r_{2}\right) \geq \sum_{i} \psi_{i} r_{i} \text { for }\left(-\varphi_{i}, \psi_{i}\right) \in \mathfrak{F}_{i} \text { with } \varphi_{1}+\varphi_{2} \leq c .
$$

We now introduce the integral functional associated with the marginal perspective cost (5.3), which is based on the Lebesgue decomposition $\mu_{i}=\varrho_{i} \gamma_{i}+\mu_{i}^{\perp}$ (see Lemma 2.3),

$$
\mathscr{H}\left(\mu_{1}, \mu_{2} \mid \boldsymbol{\gamma}\right):=\int_{X} H\left(x_{1}, \varrho_{1}\left(x_{1}\right) ; x_{2}, \varrho_{2}\left(x_{2}\right)\right) \mathrm{d} \boldsymbol{\gamma}+\sum_{i} F_{i}(0) \mu_{i}^{\perp}\left(X_{i}\right)
$$

where we adopted the same notation as in (3.27). Let us first show that $\mathscr{H}$ is always greater than $\mathscr{D}$.

Lemma 5.4 For every $\boldsymbol{\gamma} \in \mathcal{M}(\boldsymbol{X}), \mu_{i}, \mu_{i}^{\prime} \in \mathcal{M}\left(X_{i}\right), \boldsymbol{\varphi} \in \boldsymbol{\Phi}, \varrho_{i} \in \mathrm{L}_{+}^{1}\left(X_{i}, \gamma_{i}\right)$ with $\mu_{i}=\varrho_{i} \gamma_{i}+\mu_{i}^{\prime}$, we have

$$
\int_{X} H\left(x_{1}, \varrho_{1}\left(x_{1}\right) ; x_{2}, \varrho_{2}\left(x_{2}\right)\right) \mathrm{d} \boldsymbol{\gamma}+\sum_{i} F_{i}(0) \mu_{i}^{\prime}\left(X_{i}\right) \geq \mathscr{D}\left(\varphi \mid \mu_{1}, \mu_{2}\right) .
$$

Proof Recalling that $F_{i}^{\circ}\left(\varphi_{i}\right)=-F^{*}\left(-\varphi_{i}\right) \leq F_{i}(0)$ by (2.19) and (2.45), and using (5.15) with $r_{j}=\rho_{j}$ and $\psi_{j}=F_{j}^{\circ}\left(\rho_{j}\right)$ we have

$$
\begin{aligned}
& \int_{X} H\left(x_{1}, \varrho_{1}\left(x_{1}\right) ; x_{2}, \varrho_{2}\left(x_{2}\right)\right) \mathrm{d} \boldsymbol{\gamma}+\sum_{i} F_{i}(0) \mu_{i}^{\prime}\left(X_{i}\right) \\
& \stackrel{(5.15)}{\geq} \int_{X}\left(F_{1}^{\circ}\left(\varphi_{1}\left(x_{1}\right)\right) \varrho_{1}\left(x_{1}\right)+F_{2}^{\circ}\left(\varphi_{2}\left(x_{2}\right)\right) \varrho_{2}\left(x_{2}\right)\right) \mathrm{d} \boldsymbol{\gamma}+\sum_{i} F_{i}(0) \mu_{i}^{\prime}\left(X_{i}\right) \\
& \quad=\sum_{i} \int_{X_{i}} F_{i}^{\circ}\left(\varphi_{i}\right) \varrho_{i}\left(x_{i}\right) \mathrm{d} \gamma_{i}+\sum_{i} F_{i}(0) \mu_{i}^{\prime}\left(X_{i}\right)
\end{aligned}
$$




$$
\begin{aligned}
& \stackrel{(2.19)}{\geq} \sum_{i} \int_{X_{i}} F_{i}^{\circ}\left(\varphi_{i}\right) \varrho_{i}\left(x_{i}\right) \mathrm{d} \gamma_{i}+\sum_{i} \int_{X_{i}} F_{i}^{\circ}\left(\varphi_{i}\right) \mathrm{d} \mu_{i}^{\prime} \\
& =\sum_{i} \int_{X_{i}} F_{i}^{\circ}\left(\varphi_{i}\right) \mathrm{d} \mu_{i}=\mathscr{D}\left(\varphi \mid \mu_{1}, \mu_{2}\right) .
\end{aligned}
$$

An immediate consequence of the previous lemma is the following important result concerning the marginal perspective cost functional $\mathscr{H}$ defined by (5.16). It can be nicely compared to the Reverse Entropy-Transport functional $\mathscr{R}$ for which Theorem 3.11 stated $\mathscr{R}\left(\mu_{1}, \mu_{2} \mid \boldsymbol{\gamma}\right)=\mathscr{E}\left(\boldsymbol{\gamma} \mid \mu_{1}, \mu_{2}\right)$.

Theorem 5.5 For every $\mu_{i} \in \mathcal{M}\left(X_{i}\right), \boldsymbol{\gamma} \in \mathcal{M}(\boldsymbol{X})$ and $\boldsymbol{\varphi} \in \boldsymbol{\Phi}$ we have

$$
\mathscr{R}\left(\mu_{1}, \mu_{2} \mid \boldsymbol{\gamma}\right) \geq \mathscr{H}\left(\mu_{1}, \mu_{2} \mid \boldsymbol{\gamma}\right) \geq \mathscr{D}\left(\varphi \mid \mu_{1}, \mu_{2}\right)
$$

In particular

$$
\mathrm{ET}\left(\mu_{1}, \mu_{2}\right)=\mathrm{H}\left(\mu_{1}, \mu_{2}\right):=\min _{\boldsymbol{\gamma} \in \mathcal{M}(\boldsymbol{X})} \mathscr{H}\left(\mu_{1}, \mu_{2} \mid \boldsymbol{\gamma}\right),
$$

and $\boldsymbol{\gamma} \in \mathrm{Opt}_{\mathrm{ET}}\left(\mu_{1}, \mu_{2}\right)$ if and only if it minimizes $\mathscr{H}\left(\mu_{1}, \mu_{2} \mid \cdot\right)$ in $\mathcal{M}(\boldsymbol{X})$ and satisfies

$$
H\left(x_{1}, \varrho_{1}\left(x_{1}\right) ; x_{2}, \varrho_{2}\left(x_{2}\right)\right)=\sum_{i} R_{i}\left(\varrho_{i}\left(x_{i}\right)\right)+\mathrm{c}\left(x_{1}, x_{2}\right) \quad \gamma \text {-a.e. in } \boldsymbol{X},
$$

where $\varrho_{i}$ is defined as in (2.8). If moreover the following conditions

$$
\begin{aligned}
& F_{1}(0)=+\infty \text { or there exists } \bar{x}_{2} \in X_{2} \text { with } \mu_{2}\left(\left\{\bar{x}_{2}\right\}\right)=0, \\
& F_{2}(0)=+\infty \text { or there exists } \bar{x}_{1} \in X_{1} \text { with } \mu_{1}\left(\left\{\bar{x}_{1}\right\}\right)=0,
\end{aligned}
$$

are satisfied, then

$$
\begin{aligned}
\mathrm{ET}\left(\mu_{1}, \mu_{2}\right)=\min \left\{\int_{\boldsymbol{X}} H\left(x_{1}, \varrho_{1}\left(x_{1}\right) ; x_{2}, \varrho_{2}\left(x_{2}\right)\right) \mathrm{d} \boldsymbol{\gamma}:\right. & \\
& \left.\boldsymbol{\gamma} \in \mathcal{M}(\boldsymbol{X}), \mu_{i}=\varrho_{i} \gamma_{i}\right\} .
\end{aligned}
$$

Proof The inequality $\mathscr{R}\left(\mu_{1}, \mu_{2} \mid \boldsymbol{\gamma}\right) \geq \mathscr{H}\left(\mu_{1}, \mu_{2} \mid \boldsymbol{\gamma}\right)$ is an immediate consequence of the fact that $\sum_{i} R_{i}\left(r_{i}\right)+c \geq \tilde{H}_{c}\left(r_{1}, r_{2}\right) \geq H_{c}\left(r_{1}, r_{2}\right)$ for every $r_{i}, c \in[0, \infty]$, obtained by choosing $\theta=1$ in (5.1). The estimate $\mathscr{H}\left(\mu_{1}, \mu_{2} \mid \boldsymbol{\gamma}\right) \geq \mathscr{D}\left(\boldsymbol{\varphi} \mid \mu_{1}, \mu_{2}\right)$ was shown in Lemma 5.4. 
By using the "reverse" formulation of $\mathrm{ET}\left(\mu_{1}, \mu_{2}\right)$ in terms of the functional $\mathscr{R}\left(\mu_{1}, \mu_{2} \mid \boldsymbol{\gamma}\right)$ given by Theorem 3.11 and applying Theorem 4.11 we obtain (5.19) and the characterization (5.20).

To establish the identity (5.22) we note that the difference to (5.19) only lies in dropping the additional restriction $\mu_{i}^{\perp}=0$. When both $F_{1}(0)=F_{2}(0)=$ $+\infty$ the equivalence is obvious since the finiteness of the functional $\gamma \mapsto$ $\mathscr{H}\left(\mu_{1}, \mu_{2} \mid \boldsymbol{\gamma}\right)$ yields $\mu_{1}^{\perp}=\mu_{2}^{\perp}=0$.

In the general case, one immediately see that the right-hand side $E^{\prime}$ of (5.22) (with "inf" instead of "min") is larger than $E T\left(\mu_{1}, \mu_{2}\right)$, since the infimum of $\mathscr{H}\left(\mu_{1}, \mu_{2} \mid \cdot\right)$ is constrained to the smaller set of plans $\gamma$ satisfying $\mu_{i} \ll \gamma_{i}$. On the other hand, if $\bar{\gamma} \in \operatorname{Opt}_{\mathrm{ET}}\left(\mu_{1}, \mu_{2}\right)$ with $\mu_{i}=\varrho_{i} \bar{\gamma}_{i}+\mu_{i}^{\perp}$ and $\tilde{m}_{i}:=$ $\mu_{i}^{\perp}\left(X_{i}\right)>0$, we can consider $\gamma:=\bar{\gamma}+\frac{1}{\tilde{m}_{1} \tilde{m}_{2}} \mu_{1}^{\perp} \otimes \mu_{2}^{\perp}$ which satisfies $\mu_{i} \ll \gamma_{i}$; by exploiting the fact that $H\left(x_{1}, r_{1} ; x_{2}, r_{2}\right) \leq \sum_{i} F_{i}(0) r_{i}$ by (5.12), we obtain

$$
\begin{aligned}
& \mathscr{H}\left(\mu_{1}, \mu_{2} \mid \boldsymbol{\gamma}\right)= \int_{X} H\left(x_{1}, \varrho_{1}\left(x_{1}\right) ; x_{2}, \varrho_{2}\left(x_{2}\right)\right) \mathrm{d} \bar{\gamma} \\
&+\frac{1}{\tilde{m}_{1} \tilde{m}_{2}} \int_{X} H\left(x_{1}, \tilde{m}_{1} ; x_{2}, \tilde{m}_{2}\right) \mathrm{d} \mu_{1}^{\perp} \otimes \mu_{2}^{\perp} \\
& \leq \int_{X} H\left(x_{1}, \varrho_{1}\left(x_{1}\right) ; x_{2}, \varrho_{2}\left(x_{2}\right)\right) \mathrm{d} \bar{\gamma}+\sum_{i} F_{i}(0) \tilde{m}_{i}=\mathscr{H}\left(\mu_{1}, \mu_{2} \mid \bar{\gamma}\right),
\end{aligned}
$$

so that we have $E^{\prime} \leq \mathrm{ET}\left(\mu_{1}, \mu_{2}\right)$. The case when only one (say $\left.\mu_{2}^{\perp}\right)$ of the measures $\mu_{i}^{\perp}$ vanishes can be treated in the same way: since in this case $\tilde{m}_{1}=\mu_{1}^{\perp}\left(X_{1}\right)>0$ and therefore $F_{1}(0)<\infty$, by applying (5.21) we can choose $\boldsymbol{\gamma}:=\bar{\gamma}+\frac{1}{\tilde{m}_{1}} \mu_{1}^{\perp} \otimes \delta_{\bar{x}_{2}}$, obtaining

$$
\begin{aligned}
& \mathscr{H}\left(\mu_{1}, \mu_{2} \mid \boldsymbol{\gamma}\right) \\
& =\int_{X} H\left(x_{1}, \varrho_{1}\left(x_{1}\right) ; x_{2}, \varrho_{2}\left(x_{2}\right)\right) \mathrm{d} \overline{\boldsymbol{\gamma}}+\frac{1}{\tilde{m}_{1}} \int_{X_{1}} H\left(x_{1}, \tilde{m}_{1} ; \bar{x}_{2}, 0\right) \mathrm{d} \mu_{1}^{\perp} \\
& \leq \int_{X} H\left(x_{1}, \varrho_{1}\left(x_{1}\right) ; x_{2}, \varrho_{2}\left(x_{2}\right)\right) \mathrm{d} \overline{\boldsymbol{\gamma}}+F_{1}(0) \tilde{m}_{1}=\mathscr{H}\left(\mu_{1}, \mu_{2} \mid \overline{\boldsymbol{\gamma}}\right) .
\end{aligned}
$$

Remark 5.6 Notice that (5.21) is always satisfied if the spaces $X_{i}$ are uncountable. If $X_{i}$ is countable, one can always add an isolated point $\bar{x}_{i}$ (sometimes called "cemetery") to $X_{i}$ and consider the augmented space $\bar{X}_{i}=X_{i} \sqcup\left\{\bar{x}_{i}\right\}$ obtained as the disjoint union of $X$ and $\bar{x}_{i}$, with augmented cost $\overline{\mathrm{C}}$ which extends c to $+\infty$ on $\bar{X}_{1} \times \bar{X}_{2} \backslash\left(X_{1} \times X_{2}\right)$. We can recover (5.22) by allowing $\gamma$ in $\mathcal{M}\left(\bar{X}_{1} \times \bar{X}_{2}\right)$. 


\subsection{Entropy-transport problems with "homogeneous" marginal constraints}

In this section we will exploit the 1-homogeneity of the marginal perspective function $\mathscr{H}$ in order to derive a last representation of the functional ET, related to the new notion of homogeneous marginals. We will confine our presentation to the basic facts, and we will devote the second part of the paper to develop a full theory for the specific case of the Logarithmic Entropy-transport case.

In particular, the following construction (typical in the Young measure approach to variational problems) allows us to consider the entropy-transport problems in a setting of greater generality. We replace a pair $(\gamma, \varrho)$, where $\gamma$ and $\varrho$ are a measure on $X$ and a nonnegative Borel function, by a measure $\alpha \in \mathcal{M}(Y)$ on the extended space $Y=X \times[0, \infty)$. The original pair $(\gamma, \varrho)$ corresponds to a measure $\alpha=(x, \varrho(x))_{\sharp \gamma}$ concentrated on the graph of $\varrho$ in $Y$ and whose first marginal is $\gamma$.

Homogeneous marginals. In the usual setting of Sect. 3.1, we consider the product spaces $Y_{i}:=X_{i} \times[0, \infty)$ endowed with the product topology and denote the generic points in $Y_{i}$ with $y_{i}=\left(x_{i}, r_{i}\right), x_{i} \in X_{i}$ and $r_{i} \in[0, \infty)$ for $i=1,2$. Projections from $\boldsymbol{Y}:=Y_{1} \times Y_{2}$ onto the various coordinates will be denoted by $\pi^{y_{i}}, \pi^{x_{i}}, \pi^{r_{i}}$ with obvious meaning.

For $p>0$ and $\boldsymbol{y} \in \boldsymbol{Y}$ we will set $|\boldsymbol{y}|_{p}^{p}:=\sum_{i}\left|r_{i}\right|^{p}$ and call $\mathcal{M}_{p}(\boldsymbol{Y})$ (resp. $\left.\mathcal{P}_{p}(\boldsymbol{Y})\right)$ the space of measures $\boldsymbol{\alpha} \in \mathcal{M}(\boldsymbol{Y})($ resp. $\mathcal{P}(\boldsymbol{Y}))$ such that

$$
\int_{\boldsymbol{Y}}|\boldsymbol{y}|_{p}^{p} \mathrm{~d} \boldsymbol{\alpha}<\infty
$$

If $\boldsymbol{\alpha} \in \mathcal{M}_{p}(\boldsymbol{Y})$ the measures $r_{i}^{p} \boldsymbol{\alpha}$ belong to $\mathcal{M}(\boldsymbol{Y})$, which allow us to define the " $p$-homogeneous" marginal $\mathrm{h}_{i}^{p}(\boldsymbol{\alpha})$ of $\boldsymbol{\alpha} \in \mathcal{M}_{p}(\boldsymbol{Y})$ as the $x_{i}$-marginal of $r_{i}^{p} \boldsymbol{\alpha}$, namely

$$
\mathrm{h}_{i}^{p}(\boldsymbol{\alpha}):=\pi_{\sharp}^{x_{i}}\left(r_{i}^{p} \boldsymbol{\alpha}\right) \in \mathcal{M}\left(X_{i}\right) .
$$

The maps $\mathrm{h}_{i}^{p}: \mathcal{M}_{p}(\boldsymbol{Y}) \rightarrow \mathcal{M}\left(X_{i}\right)$ are linear and invariant with respect to dilations: if $\vartheta: \boldsymbol{Y} \rightarrow(0, \infty)$ is a Borel map in $\mathrm{L}^{p}(\boldsymbol{Y}, \boldsymbol{\alpha})$ and $\operatorname{prd}_{\vartheta}(\boldsymbol{y}):=$ $\left(x_{1}, r_{1} / \vartheta(\boldsymbol{y}) ; x_{2}, r_{2} / \vartheta(\boldsymbol{y})\right)$, we set

$$
\begin{aligned}
& \operatorname{dil}_{\vartheta, p}(\boldsymbol{\alpha}):=\left(\operatorname{prd}_{\vartheta}\right)_{\sharp}\left(\vartheta^{p} \boldsymbol{\alpha}\right), \text { i.e. for } \varphi \in \mathrm{B}_{b}(\boldsymbol{Y}) \\
& \int \varphi(\boldsymbol{y}) \mathrm{d}\left(\operatorname{dil}_{\vartheta, p}(\boldsymbol{\alpha})\right)=\int \varphi\left(x_{1}, r_{1} / \vartheta ; x_{2}, r_{2} / \vartheta\right) \vartheta^{p}(\boldsymbol{y}) \mathrm{d} \boldsymbol{\alpha}(\boldsymbol{y}) .
\end{aligned}
$$


Using (5.24) we obviously have

$$
\mathrm{h}_{i}^{p}\left(\operatorname{dil}_{\vartheta, p}(\boldsymbol{\alpha})\right)=\mathrm{h}_{i}^{p}(\boldsymbol{\alpha}) .
$$

In particular, for $\boldsymbol{\alpha} \in \mathcal{M}_{p}(\boldsymbol{Y})$ with $\boldsymbol{\alpha}(\boldsymbol{Y})>0$, by choosing

$$
\vartheta(\boldsymbol{y}):=\frac{1}{r_{*}}\left\{\begin{array}{ll}
|\boldsymbol{y}|_{p} & \text { if }|\boldsymbol{y}|_{p} \neq 0, \\
1 & \text { if }|\boldsymbol{y}|_{p}=0,
\end{array} \quad r_{*}:=\left(\int_{\boldsymbol{Y}}|\boldsymbol{y}|_{p}^{p} \mathrm{~d} \boldsymbol{\alpha}+\boldsymbol{\alpha}\left(\left\{|\boldsymbol{y}|_{p}=0\right\}\right)\right)^{1 / p}\right.
$$

we obtain a rescaled probability measure $\tilde{\alpha}$ with the same homogeneous marginals as $\boldsymbol{\alpha}$ and concentrated on $\boldsymbol{Y}_{r_{*}, p}:=\left\{\boldsymbol{y} \in \boldsymbol{Y}:|\boldsymbol{y}|_{p} \leq r_{*}\right\} \subset$ $\left(X \times\left[0, r_{*}\right]\right) \times\left(X \times\left[0, r_{*}\right]\right)$ :

$$
\tilde{\boldsymbol{\alpha}}=\operatorname{dil}_{\vartheta, p}(\boldsymbol{\alpha}) \in \mathcal{P}_{p}(\boldsymbol{Y}), \quad \mathrm{h}_{i}^{p}(\tilde{\boldsymbol{\alpha}})=\mathrm{h}_{i}^{p}(\boldsymbol{\alpha}), \quad \tilde{\boldsymbol{\alpha}}\left(\boldsymbol{Y} \backslash \boldsymbol{Y}_{r_{*}, p}\right)=0 .
$$

Entropy-transport problems with prescribed homogeneous marginals. Given $\mu_{1}, \mu_{2} \in \mathcal{M}(X)$ we now introduce the convex sets

$$
\begin{aligned}
& \mathcal{H}_{\leq}^{p}\left(\mu_{1}, \mu_{2}\right):=\left\{\boldsymbol{\alpha} \in \mathcal{M}_{p}(\boldsymbol{Y}): \mathrm{h}_{i}^{p}(\boldsymbol{\alpha}) \leq \mu_{i}\right\}, \\
& \mathcal{H}_{\underline{p}}^{p}\left(\mu_{1}, \mu_{2}\right):=\left\{\boldsymbol{\alpha} \in \mathcal{M}_{p}(\boldsymbol{Y}): \mathrm{h}_{i}^{p}(\boldsymbol{\alpha})=\mu_{i}\right\} .
\end{aligned}
$$

Clearly $\mathcal{H}_{\underline{p}}^{p}\left(\mu_{1}, \mu_{2}\right) \subset \mathcal{H}_{\leq}^{p}\left(\mu_{1}, \mu_{2}\right)$ and they are nonempty since every plan of the form

$$
\alpha=\frac{1}{a_{1}^{p} a_{2}^{p}}\left(\mu_{1} \otimes \delta_{a_{1}}\right) \otimes\left(\mu_{2} \otimes \delta_{a_{2}}\right), \quad \text { with } a_{1}, a_{2}>0
$$

belongs to $\mathcal{H}_{\underline{\underline{p}}}^{\underline{p}}\left(\mu_{1}, \mu_{2}\right)$. It is not difficult to check that $\mathcal{H}_{\leq}^{p}\left(\mu_{1}, \mu_{2}\right)$ is also narrowly closed, while, on the contrary, this property fails for $\mathcal{H} \underline{\underline{p}}\left(\mu_{1}, \mu_{2}\right)$ if $\mu_{1}\left(X_{1}\right) \mu_{2}\left(X_{2}\right) \neq 0$. To see this, it is sufficient to consider any $\boldsymbol{\alpha} \in$ $\mathcal{H} \underline{\underline{p}}\left(\mu_{1}, \mu_{2}\right) \backslash\{0\}$ and look at the vanishing sequence $\operatorname{dil}_{n^{-1}, p}(\boldsymbol{\alpha})$ for $n \rightarrow \infty$.

There is a natural correspondence between $\mathcal{H}_{\leq}^{p}\left(\mu_{1}, \mu_{2}\right)\left(\operatorname{resp} . \mathcal{H}_{\underline{\underline{p}}}^{p}\left(\mu_{1}, \mu_{2}\right)\right)$ and $\mathcal{H}_{<}^{1}\left(\mu_{1}, \mu_{2}\right)\left(\operatorname{resp} . \mathcal{H}_{=}^{1}\left(\mu_{1}, \mu_{2}\right)\right)$ induced by the map $\boldsymbol{Y} \ni\left(x_{1}, r_{1} ; x_{2}, r_{2}\right) \mapsto$ $\left(x_{1}, r_{1}^{\bar{p}} ; x_{2}, r_{2}^{p}\right)$. For plans $\boldsymbol{\alpha} \in \mathcal{H}_{\leq}^{1}\left(\mu_{1}, \mu_{2}\right)$ we can prove a result similar to Lemma 5.4 but now we obtain a linear functional in $\boldsymbol{\alpha}$.

Lemma 5.7 For $p \in(0, \infty), \mu_{i} \in \mathcal{M}\left(X_{i}\right), \boldsymbol{\varphi} \in \boldsymbol{\Phi}$, and $\boldsymbol{\alpha} \in \mathcal{H}_{\leq}^{p}\left(\mu_{1}, \mu_{2}\right)$ we have

$$
\begin{aligned}
& \int_{\boldsymbol{X}} H\left(x_{1}, r_{1}^{p} ; x_{2}, r_{2}^{p}\right) \mathrm{d} \boldsymbol{\alpha}+\sum_{i} F_{i}(0) \mu_{i}^{\prime}\left(X_{i}\right) \geq \mathscr{D}\left(\boldsymbol{\varphi} \mid \mu_{1}, \mu_{2}\right), \\
& \text { where } \mu_{i}^{\prime}:=\mu_{i}-\mathrm{h}_{i}^{p}(\boldsymbol{\alpha}) .
\end{aligned}
$$


Proof The calculations are quite similar to the proof of Lemma 5.4:

$$
\begin{aligned}
& \int_{\boldsymbol{Y}} H\left(x_{1}, r_{1}^{p} ; x_{2}, r_{2}^{p}\right) \mathrm{d} \boldsymbol{\alpha}+\sum_{i} F_{i}(0) \mu_{i}^{\prime}\left(X_{i}\right) \\
& \stackrel{(5.15)}{\geq} \int_{\boldsymbol{Y}}\left(F_{1}^{\circ}\left(\varphi_{1}\left(x_{1}\right)\right) r_{1}^{p}+F_{2}^{\circ}\left(\varphi_{2}\left(x_{2}\right)\right) r_{2}^{p}\right) \mathrm{d} \boldsymbol{\alpha}+\sum_{i} F_{i}(0) \mu_{i}^{\prime}\left(X_{i}\right) \\
& \quad=\sum_{i} \int_{X_{i}} F_{i}^{\circ}\left(\varphi_{i}\right) \mathrm{d}\left(\mathrm{h}_{i}^{p}(\boldsymbol{\alpha})\right)+\sum_{i} F_{i}(0) \mu_{i}^{\prime}\left(X_{i}\right) \\
& \quad \stackrel{(2.19)}{\geq} \sum_{i} \int_{X_{i}} F_{i}^{\circ}\left(\varphi_{i}\right) \mathrm{d}\left(\mathrm{h}_{i}^{p}(\boldsymbol{\alpha})\right)+\sum_{i} \int_{X_{i}} F_{i}^{\circ}\left(\varphi_{i}\right) \mathrm{d} \mu_{i}^{\prime} \\
& =\sum_{i} \int_{X_{i}} F_{i}^{\circ}\left(\varphi_{i}\right) \mathrm{d} \mu_{i}=\mathscr{D}\left(\varphi \mid \mu_{1}, \mu_{2}\right) .
\end{aligned}
$$

As a consequence, we can characterize the entropy-transport minimum via measures $\boldsymbol{\alpha} \in \mathcal{M}(\boldsymbol{Y})$.

Theorem 5.8 For every $\mu_{i} \in \mathcal{M}\left(X_{i}\right), p \in(0, \infty)$ we have

$$
\begin{aligned}
& \operatorname{ET}\left(\mu_{1}, \mu_{2}\right)=\min _{\boldsymbol{\alpha} \in \mathcal{H}_{\leq}^{p}\left(\mu_{1}, \mu_{2}\right)} \int_{\boldsymbol{Y}}\left(\sum_{i} R_{i}\left(r_{i}^{p}\right)+\mathrm{c}\left(x_{1}, x_{2}\right)\right) \mathrm{d} \boldsymbol{\alpha} \\
& +\sum_{i} F_{i}(0)\left(\mu_{i}-\mathrm{h}_{i}^{p}(\boldsymbol{\alpha})\right)\left(X_{i}\right) \\
& =\min _{\boldsymbol{\alpha} \in \mathcal{H}_{\leq}^{p}\left(\mu_{1}, \mu_{2}\right)} \int_{\boldsymbol{Y}} H\left(x_{1}, r_{1}^{p} ; x_{2}, r_{2}^{p}\right) \mathrm{d} \boldsymbol{\alpha}+\sum_{i} F_{i}(0)\left(\mu_{i}-\mathrm{h}_{i}^{p}(\boldsymbol{\alpha})\right)\left(X_{i}\right) \\
& =\min _{\boldsymbol{\alpha} \in \mathcal{H}_{\underline{\underline{p}}}^{\underline{p}}\left(\mu_{1}, \mu_{2}\right)} \int_{\boldsymbol{Y}} H\left(x_{1}, r_{1}^{p} ; x_{2}, r_{2}^{p}\right) \mathrm{d} \boldsymbol{\alpha} .
\end{aligned}
$$

Moreover, for every plan $\gamma \in \mathrm{Opt}_{\mathrm{ET}}\left(\mu_{1}, \mu_{2}\right)$ (resp. optimal for (5.19) or for (5.22)) with $\mu_{i}=\varrho_{i} \gamma_{i}+\mu_{i}^{\perp}$, the plan $\alpha:=\left(x_{1}, \varrho_{1}^{1 / p}\left(x_{1}\right) ; x_{2}, \varrho_{2}^{1 / p}\left(x_{2}\right)\right)_{\sharp} \gamma$ realizes the minimum of (5.31) (resp. (5.32) or (5.33)). 
Remark 5.9 When $F_{i}(0)=+\infty(5.31)$ and (5.32) simply read as

$$
\begin{aligned}
\mathrm{ET}\left(\mu_{1}, \mu_{2}\right) & =\min _{\boldsymbol{\alpha} \in \mathcal{H} \stackrel{p}{\underline{p}}\left(\mu_{1}, \mu_{2}\right)} \int_{\boldsymbol{Y}}\left(\sum_{i} R_{i}\left(r_{i}^{p}\right)+\mathrm{c}\left(x_{1}, x_{2}\right)\right) \mathrm{d} \boldsymbol{\alpha} \\
& =\min _{\boldsymbol{\alpha} \in \mathcal{H} \stackrel{1}{=}\left(\mu_{1}, \mu_{2}\right)} \int_{\boldsymbol{Y}} H\left(x_{1}, r_{1}^{p} ; x_{2}, r_{2}^{p}\right) \mathrm{d} \boldsymbol{\alpha} .
\end{aligned}
$$

Proof of Theorem 5.8 Let us denote by $E^{\prime}$ (resp. $E^{\prime \prime}, E^{\prime \prime \prime}$ ) the right-hand side of (5.31) (resp. of (5.32), (5.33)), where "min" has been replaced by "inf". If $\boldsymbol{\gamma} \in \mathcal{M}(\boldsymbol{X})$ and $\mu_{i}=\varrho_{i} \gamma_{i}+\mu_{i}^{\perp}$ (in the case of (5.33) $\mu_{i}^{\perp}=0$ ) is the usual Lebesgue decomposition as in Lemma 2.3, we can consider the plan $\boldsymbol{\alpha}:=\left(x_{1}, \varrho_{1}^{1 / p}\left(x_{1}\right) ; x_{2}, \varrho_{2}^{1 / p}\left(x_{2}\right)\right)_{\sharp} \boldsymbol{\gamma}$.

Since the map $\left(\varrho_{1}^{1 / p}, \varrho_{2}^{1 / p}\right): \boldsymbol{X} \rightarrow \mathbb{R}^{2}$ is Borel and takes values in a metrizable and separable space, it is Lusin $\boldsymbol{\gamma}$-measurable [44, Thm 5, p. 26], so that $\boldsymbol{\alpha}$ is a Radon measure in $\mathcal{M}(\boldsymbol{Y})$. For every nonnegative $\phi_{i} \in \mathrm{B}_{b}\left(X_{i}\right)$ we easily get

$$
\int \phi_{i}\left(x_{i}\right) r_{i}^{p} \mathrm{~d} \boldsymbol{\alpha}=\int \varrho_{i}\left(x_{i}\right) \phi_{i}\left(x_{i}\right) \mathrm{d} \boldsymbol{\gamma}=\int \varrho_{i} \phi_{i} \mathrm{~d} \gamma_{i} \leq \int \phi_{i} \mathrm{~d} \mu_{i},
$$

so that $\boldsymbol{\alpha} \in \mathcal{H}_{\leq}^{p}\left(\mu_{1}, \mu_{2}\right), \mathrm{h}_{i}^{p}(\boldsymbol{\alpha})=\varrho_{i} \gamma_{i}$, and

$$
\begin{aligned}
\mathscr{R}\left(\mu_{1}, \mu_{2} \mid \boldsymbol{\gamma}\right)= & \int_{X}\left(\sum_{i} R_{i}\left(\varrho_{i}\left(x_{i}\right)\right)+\mathrm{c}\left(x_{1}, x_{2}\right)\right) \mathrm{d} \boldsymbol{\gamma}+\sum_{i} F_{i}(0) \mu_{i}^{\perp}\left(X_{i}\right) \\
= & \left.\int_{\boldsymbol{Y}} \sum_{i} R_{i}\left(r_{i}^{p}\right)+\mathrm{c}\left(x_{1}, x_{2}\right)\right) \mathrm{d} \boldsymbol{\alpha} \\
& +\sum_{i} F_{i}(0)\left(\mu_{i}-\mathrm{h}_{i}^{p}(\boldsymbol{\alpha})\right)\left(X_{i}\right) \geq E^{\prime} .
\end{aligned}
$$

Taking the infimum w.r.t. $\boldsymbol{\gamma}$ and recalling (3.30) we get $\mathrm{ET}\left(\mu_{1}, \mu_{2}\right) \geq E^{\prime}$. Since $\sum_{i} R_{i}\left(r_{i}^{p}\right)+\mathrm{c}\left(x_{1}, x_{2}\right) \geq H\left(x_{1}, r_{1}^{p} ; x_{2}, r_{2}^{p}\right)$ it is also clear that $E^{\prime} \geq E^{\prime \prime}$.

On the other hand, Lemma 5.7 shows that $E^{\prime \prime} \geq \mathscr{D}\left(\varphi \mid \mu_{1}, \mu_{2}\right)$ for every $\boldsymbol{\varphi} \in \boldsymbol{\Phi}$. Applying Theorem 4.11 we get ET $\left(\mu_{1}, \mu_{2}\right)=E^{\prime}=E^{\prime \prime}$.

Concerning $E^{\prime \prime \prime}$ it is clear that $E^{\prime \prime \prime} \geq E^{\prime \prime}=\mathrm{ET}\left(\mu_{1}, \mu_{2}\right)$. When (5.21) hold, by choosing $\alpha$ induced by a minimizer of (5.22) we get the opposite inequality $E^{\prime \prime \prime} \leq \mathrm{ET}\left(\mu_{1}, \mu_{2}\right)$.

If (5.21) does not hold, we can still apply a slight modification of the argument at the end of the proof of Theorem 5.5. The only case to consider is when only one of the two measures $\mu_{i}^{\perp}$ vanishes: just to fix the ideas, let us suppose that $\tilde{m}_{1}=\mu_{1}^{\perp}\left(X_{1}\right)>0=\mu_{2}^{\perp}\left(X_{2}\right)$. If $\bar{\gamma} \in \mathrm{Opt}_{\mathrm{ET}}\left(\mu_{1}, \mu_{2}\right)$ and $\bar{\alpha}$ is obtained 
as above, we can just set $\boldsymbol{\alpha}:=\overline{\boldsymbol{\alpha}}+\left(\mu_{1}^{\perp} \times \delta_{1}\right) \times\left(v \times \delta_{0}\right)$ for an arbitrary $v \in \mathcal{P}\left(X_{2}\right)$. It is clear that $\mathrm{h}_{i}^{p}(\boldsymbol{\alpha})=\mu_{i}$ and

$$
\begin{aligned}
& \int_{\boldsymbol{Y}} H\left(x_{1}, r_{1}^{p} ; x_{2}, r_{2}^{p}\right) \mathrm{d} \boldsymbol{\alpha}=\int_{\boldsymbol{X}} H\left(x_{1}, \varrho_{1}\left(x_{1}\right) ; x_{2}, \varrho_{2}\left(x_{2}\right)\right) \mathrm{d} \overline{\boldsymbol{\gamma}} \\
& +\int_{X} H\left(x_{1}, 1 ; x_{2}, 0\right) \mathrm{d} \mu_{1}^{\perp} \otimes v \\
& \stackrel{(5.12)}{\leq} \int_{X} H\left(x_{1}, \varrho_{1}\left(x_{1}\right) ; x_{2}, \varrho_{2}\left(x_{2}\right)\right) \mathrm{d} \bar{\gamma}+F_{1}(0) \tilde{m}_{1} \\
& =\mathscr{H}\left(\mu_{1}, \mu_{2} \mid \bar{\gamma}\right)=\mathrm{ET}\left(\mu_{1}, \mu_{2}\right) \text {, }
\end{aligned}
$$

which yields $E^{\prime \prime \prime} \leq \mathrm{ET}\left(\mu_{1}, \mu_{2}\right)$.

Remark 5.10 (Rescaling invariance) By recalling (5.27a,b) and exploiting the 1-homogeneity of $H$ it is not restrictive to solve the minimum problem (5.32) in the smaller class of probability plans concentrated in

$$
\boldsymbol{Y}_{r, p}:=\left\{\left(x_{1}, r_{1} ; x_{2}, r_{2}\right) \in \boldsymbol{Y}: r_{1}^{p}+r_{2}^{p} \leq r^{p}\right\}, \quad r^{p}=\sum_{i} \mu_{i}\left(X_{i}\right)
$$

First, we note that it is not restrictive to assume that $\alpha\left(\left\{\boldsymbol{y} \in \boldsymbol{Y}:|\boldsymbol{y}|_{p}=\right.\right.$ $0\})=0$ in (5.32): we can always replace $\boldsymbol{\alpha}$ with $\boldsymbol{\alpha}^{\prime}:=\boldsymbol{\alpha}\left\llcorner\left\{\boldsymbol{y} \in \boldsymbol{Y}:|\boldsymbol{y}|_{p}>0\right\}\right.$ since $H\left(x_{1}, 0 ; x_{2}, 0\right)=0$ for every $x_{i} \in X_{i}$ and the homogeneous marginals of $\boldsymbol{\alpha}$ and $\boldsymbol{\alpha}^{\prime}$ coincide. As a second step, for every $\boldsymbol{\alpha} \in \mathcal{H}_{\leq}^{p}\left(\mu_{1}, \mu_{2}\right)$ with $\boldsymbol{\alpha}(\boldsymbol{Y})>0$, the choice $\tilde{\alpha}$ given by $(5.27 \mathrm{a}, \mathrm{b})$ yields a new probability plan concentrated on $\boldsymbol{Y}_{r_{*}, p} \subset \boldsymbol{Y}_{r, p}$ with the same homogeneous marginals as $\boldsymbol{\alpha}$ and satisfying

$$
\begin{aligned}
\int_{\boldsymbol{Y}} H\left(x_{1}, r_{1}^{p} ; x_{2}, r_{2}^{p}\right) \mathrm{d} \boldsymbol{\alpha} & =\int_{\boldsymbol{Y}} H\left(x_{1},\left(r_{1} / \vartheta\right)^{p} ; x_{2},\left(r_{2} / \vartheta\right)^{p}\right) \vartheta^{p} \mathrm{~d} \overline{\boldsymbol{\alpha}} \\
& =\int_{\boldsymbol{Y}} H\left(x_{1}, r_{1}^{p} ; x_{2}, r_{2}^{p}\right) \mathrm{d} \overline{\boldsymbol{\alpha}},
\end{aligned}
$$

where $\vartheta$ is the function defined in (5.27a) and we used the 1-homogeneity of $H$ w.r.t. the variables $\left(r_{1}, r_{2}\right)$.

Part II. The Logarithmic Entropy-Transport problem and the HellingerKantorovich distance

\section{The Logarithmic Entropy-Transport (LET) problem}

Starting from this section we will study a particular Entropy-Transport problem, whose structure reveals surprising properties. 


\subsection{The metric setting for Logarithmic Entropy-Transport problems}

Let $(X, \tau)$ be a Hausdorff topological space endowed with an extended distance function $\mathrm{d}: X \times X \rightarrow[0, \infty]$ which is lower semicontinuous w.r.t. $\tau$; we refer to $(X, \tau, \mathrm{d})$ as an extended metric-topological space. In the most common situations, $\mathrm{d}$ will take finite values, $(X, \mathrm{~d})$ will be separable and complete and $\tau$ will be the topology induced by $\mathrm{d}$; nevertheless, there are interesting applications where nonseparable extended distances play an important role, so that it will be useful to deal with an auxiliary topology, see e.g. $[1,3]$.

From now on we suppose that $X_{1}=X_{2}=X$. We choose the logarithmic entropies

$$
F_{i}(s)=U_{1}(s):=s \log s-s+1,
$$

and a cost $\mathrm{c}$ depending on the distance $\mathrm{d}$ through the function $\ell:[0, \infty] \rightarrow$ $[0, \infty]$ via

$$
\mathrm{c}\left(x_{1}, x_{2}\right):=\ell\left(\mathrm{d}\left(x_{1}, x_{2}\right)\right), \quad \ell(d):= \begin{cases}\log \left(1+\tan ^{2}(d)\right) & \text { if } d \in[0, \pi / 2), \\ +\infty & \text { if } d \geq \pi / 2,\end{cases}
$$

so that

$$
\mathrm{c}\left(x_{1}, x_{2}\right)= \begin{cases}-\log \left(\cos ^{2}\left(\mathrm{~d}\left(x_{1}, x_{2}\right)\right)\right) & \text { if } \mathrm{d}\left(x_{1}, x_{2}\right)<\pi / 2 \\ +\infty & \text { otherwise }\end{cases}
$$

Let us collect a few key properties that will be relevant in the sequel.

LE.1 $F_{i}$ are superlinear, $\mathrm{C}^{\infty}$ in $(0, \infty)$, strictly convex, with $\mathrm{D}\left(F_{i}\right)=[0, \infty)$, $F_{i}(0)=1$, and $\left(F_{i}\right)_{0}^{\prime}=-\infty$. For $s>0$ we have $\partial F_{i}(s)=\{\log s\}$.

LE. $2 R_{i}(r)=r F_{i}(1 / r)=r-1-\log r, R_{i}(0)=+\infty,\left(R_{i}\right)_{\infty}^{\prime}=1$.

LE.3 $F_{i}^{*}(\phi)=\exp (\phi)-1, F_{i}^{\circ}(\varphi)=1-\exp (-\varphi), \mathrm{D}\left(F_{i}^{*}\right)=\mathrm{D}\left(F_{i}^{\circ}\right)=\mathbb{R}$.

LE.4 $R_{i}^{*}(\psi)=-\log (1-\psi)$ for $\psi<1$ and $R_{i}^{*}(\psi)=+\infty$ for $\psi \geq 1$.

LE.5 The function $\ell$ can be characterized as the unique solution of the differential equation

$$
\ell^{\prime \prime}(d)=2 \exp (\ell(d)), \quad \ell(0)=\ell^{\prime}(0)=0,
$$

since it satisfies

$$
\ell(d)=-\log \left(\cos ^{2}(d)\right)=2 \int_{0}^{d} \tan (s) \mathrm{d} s, \quad d \in[0, \pi / 2),
$$


so that

$$
\ell(d) \geq d^{2}, \quad \ell^{\prime}(d)=2 \tan d \geq 2 d, \quad \ell^{\prime \prime}(d) \geq 2 .
$$

In particular $\ell$ is strictly increasing and uniformly 2-convex. It is not difficult to check that $\sqrt{\ell}$ is also convex: this property is equivalent to $2 \ell \ell^{\prime \prime} \geq\left(\ell^{\prime}\right)^{2}$ and a direct calculation shows

$$
2 \ell \ell^{\prime \prime}-\left(\ell^{\prime}\right)^{2}=4 \log \left(1+\tan ^{2}(d)\right)\left(1+\tan ^{2}(d)\right)-4 \tan ^{2}(d) \geq 0
$$

since $(1+r) \log (1+r) \geq r$.

LE.6 $H_{c}\left(r_{1}, r_{2}\right)=r_{1}+r_{2}-2 \sqrt{r_{1} r_{2}} \exp (-c / 2)$ for $c<\infty$, so that

$$
H\left(x_{1}, r_{1} ; x_{2}, r_{2}\right)=r_{1}+r_{2}-2 \sqrt{r_{1} r_{2}} \cos \left(\mathrm{d}_{\pi / 2}\left(x_{1}, x_{2}\right)\right),
$$

where we set

$$
\mathrm{d}_{a}\left(x_{1}, x_{2}\right):=\mathrm{d}\left(x_{1}, x_{2}\right) \wedge a \text { for } x_{i} \in X, a \geq 0 .
$$

Since the function

$$
H\left(x_{1}, r_{1}^{2} ; x_{2}, r_{2}^{2}\right)=r_{1}^{2}+r_{2}^{2}-2 r_{1} r_{2} \cos \left(\mathrm{d}_{\pi / 2}\left(x_{1}, x_{2}\right)\right)
$$

will have an important geometric interpretation (see Sect. 7.1), in the following we will choose the exponent $p=2$ in the setting of Sect. 5.2.

We keep the usual notation $X=X \times X$, identifying $X_{1}$ and $X_{2}$ with $X$ and letting the index $i$ run between 1 and 2, e.g. for $\gamma \in \mathcal{N}(X)$ the marginals are denoted by $\gamma_{i}=\left(\pi^{i}\right)_{\sharp} \boldsymbol{\gamma}$.

Problem 6.1 (The Logarithmic Entropy-Transport problem) Let $(X, \tau, \mathrm{d})$ be an extended metric-topological space, $\ell$ and $\mathrm{c}$ be as in (6.2). Given $\mu_{i} \in \mathcal{M}(X)$ find $\boldsymbol{\gamma} \in \mathcal{M}(\boldsymbol{X})$ minimizing

$$
\operatorname{LET}\left(\mu_{1}, \mu_{2}\right)=\min _{\boldsymbol{\gamma} \in \mathcal{M}(\boldsymbol{X})}\left(\sum_{i} \int_{X}\left(\sigma_{i} \log \sigma_{i}-\sigma_{i}+1\right) \mathrm{d} \mu_{i}+\int_{\boldsymbol{X}} \ell\left(\mathrm{d}\left(x_{1}, x_{2}\right)\right) \mathrm{d} \boldsymbol{\gamma}\right),
$$

where $\sigma_{i}=\frac{\mathrm{d} \gamma_{i}}{\mathrm{~d} \mu_{i}}$. We denote by $\mathrm{Opt}_{\mathrm{ET}}\left(\mu_{1}, \mu_{2}\right)$ the set of all the minimizers $\gamma$ in (6.10).

\subsection{The Logarithmic Entropy-Transport problem: main results}

In the next theorem we collect the main properties of the Logarithmic EntropyTransport (LET) problem relying on the reverse function $\mathscr{R}$ from Sect. 3.5, cf. (3.28), and $\mathscr{H}$ from Sect. 5.1, cf. (5.16). 
Theorem 6.2 (Direct formulation of the LET problem) Let $\mu_{i} \in \mathcal{M}(X)$ be given and let $\ell, \mathrm{d}_{\pi / 2}$ be defined as in (6.2) and (6.8).

(a) Existence of optimal plans. There exists an optimal plan $\gamma \in \operatorname{Opt}_{\mathbb{E T}}\left(\mu_{1}, \mu_{2}\right)$ solving Problem 6.1. The set $\mathrm{Opt}_{\mathrm{ET}}\left(\mu_{1}, \mu_{2}\right)$ is convex and compact in $\mathcal{M}(\boldsymbol{X})$, LET is a convex and positively 1-homogeneous functional (see (4.41)) satisfying $0 \leq \mathbb{L T T}\left(\mu_{1}, \mu_{2}\right) \leq \sum_{i} \mu_{i}(X)$.

(b) Reverse formulation ( $\mathrm{ET}=\min \mathscr{R}_{\mathrm{E}}$ ). The functional $\mathrm{LT}$ has the equivalent reverse formulation as

$\operatorname{LET}\left(\mu_{1}, \mu_{2}\right)=\min \left\{\mathscr{R}_{\mathrm{LE}}\left(\mu_{1}, \mu_{2} \mid \boldsymbol{\gamma}\right): \boldsymbol{\gamma} \in \mathcal{M}(\boldsymbol{X}), \mu_{i}=\varrho_{i} \gamma_{i}+\mu_{i}^{\perp}\right\}$,

where

$$
\begin{aligned}
\mathscr{R}_{\mathrm{LE}}\left(\mu_{1}, \mu_{2} \mid \boldsymbol{\gamma}\right):=\sum_{i}\left(\mu_{i}^{\perp}(X)\right. & \left.+\int_{X}\left(\varrho_{i}-1-\log \varrho_{i}\right) \mathrm{d} \gamma_{i}\right) \\
& +\int_{X} \ell\left(\mathrm{d}\left(x_{1}, x_{2}\right)\right) \mathrm{d} \boldsymbol{\gamma},
\end{aligned}
$$

and $\bar{\gamma}$ is an optimal plan in $\mathrm{Opt}_{\mathbb{E T}}\left(\mu_{1}, \mu_{2}\right)$ if and only if it minimizes (6.11).

(c) The homogeneous perspective formulation $\left(\mathrm{LT}=\min \mathscr{H}_{\mathrm{E}}\right)$. The functional $\mathrm{LT}\left(\mu_{1}, \mu_{2}\right)$ can be equivalently characterized as

$$
\begin{aligned}
& \text { LET }\left(\mu_{1}, \mu_{2}\right)=\min \left\{\mathscr{H}_{\mathrm{E}}\left(\mu_{1}, \mu_{2} \mid \boldsymbol{\gamma}\right): \boldsymbol{\gamma} \in \mathcal{M}(\boldsymbol{X})\right\} \text {, where } \\
& \qquad \mathscr{H}_{\mathrm{E}}\left(\mu_{1}, \mu_{2} \mid \boldsymbol{\gamma}\right):=\sum_{i} \mu_{i}(X)-2 \int_{X} \sqrt{\varrho_{1}\left(x_{1}\right) \varrho_{2}\left(x_{2}\right)} \cos \left(\mathrm{d}_{\pi / 2}\left(x_{1}, x_{2}\right)\right) \mathrm{d} \boldsymbol{\gamma} \\
& =\sum_{i} \mu_{i}^{\perp}(X)+\int_{X}\left(\varrho_{1}\left(x_{1}\right)+\varrho_{2}\left(x_{2}\right)-2 \sqrt{\varrho_{1}\left(x_{1}\right) \varrho_{2}\left(x_{2}\right)} \cos \left(\mathrm{d}_{\pi / 2}\left(x_{1}, x_{2}\right)\right)\right) \mathrm{d} \boldsymbol{\gamma}
\end{aligned}
$$

and $\gamma_{i}=\varrho_{i} \mu_{i}+\mu_{i}^{\perp}$. Moreover, every plan $\bar{\gamma} \in \mathrm{Opt}_{\mathbb{E T}}\left(\mu_{1}, \mu_{2}\right)$ provides a solution to (6.12).

Proof The variational problem (6.10) fits in the class considered by Problem 3.1, in the basic coercive setting of Sect. 3.1 since the logarithmic entropy (6.1) is superlinear with domain $[0, \infty)$. The problem is always feasible since $U_{1}(0)=1$ so that (3.6) holds.

(a) follows by Theorem 3.3(i); the upper bound of LET is a particular case of (3.7), and its convexity and 1-homogeneity follows by Corollary 4.13.

(b) is a consequence of Theorem 3.11. 
(c) is an application of Theorem 5.5 and (6.7).

We consider now the dual representation of $\mathrm{ET}$; recall that $\operatorname{LSC}_{S}(X)$ denotes the space of simple (i.e. taking a finite number of values) lower semicontinuous functions and for a pair $\phi_{i}: X \rightarrow \mathbb{R}$ the symbol $\phi_{1} \oplus \phi_{2}$ denotes the function $\left(x_{1}, x_{2}\right) \mapsto \phi_{1}\left(x_{1}\right)+\phi_{2}\left(x_{2}\right)$ defined in $\boldsymbol{X}$. In part a) we relate to Sect. 4.2, whereas b)-d) discusses the optimality conditions from Sect. 4.4.

Theorem 6.3 (Dual formulation and optimality conditions)

(a) The dual problem ( $\mathrm{ET}=\mathrm{D}_{\mathrm{EE}}=\mathrm{D}_{\mathrm{EF}}^{\prime}$ ). For all $\mu_{1}, \mu_{2} \in \mathcal{M}(X)$ we have

$$
\begin{aligned}
\text { LFT } & \left(\mu_{1}, \mu_{2}\right)=\sup \left\{\mathscr{D} \mathrm{E}\left(\varphi \mid \mu_{1}, \mu_{2}\right): \varphi_{i} \in \operatorname{LSC}_{s}(X), \varphi_{1} \oplus \varphi_{2} \leq \ell(\mathrm{d})\right\}, \\
= & \sup \left\{\sum_{i} \int_{X} \psi_{i} \mathrm{~d} \mu_{i}: \psi_{i} \in \operatorname{LSC}_{S}(X), \sup _{X} \psi_{i}<1\right. \\
& \left.\left(1-\psi_{1}\left(x_{1}\right)\right)\left(1-\psi_{2}\left(x_{2}\right)\right) \geq \cos ^{2}\left(\mathrm{~d}_{\pi / 2}\left(x_{1}, x_{2}\right)\right) \text { in } X\right\}
\end{aligned}
$$

where $\mathscr{D}_{\mathrm{EE}}\left(\varphi \mid \mu_{1}, \mu\right):=\sum_{i} \int_{X}\left(1-\mathrm{e}^{-\varphi_{i}}\right) \mathrm{d} \mu_{i}$. The same identities hold if the space $\operatorname{LSC}_{s}(X)$ is replaced by $\operatorname{LSC}_{b}(X)$ or $\mathrm{B}_{b}(X)$ in (6.13) and (6.14). When the topology $\tau$ is completely regular (in particular when $\mathrm{d}$ is a distance and $\tau$ is induced by $\mathrm{d}$ ) the space $\operatorname{LSC}_{S}(X)$ can be replaced by $\mathrm{C}_{b}(X)$ as well.

(b) Optimality conditions. Let us assume that $\mathrm{d}$ is continuous. A plan $\gamma \in$ $\mathcal{M}(\boldsymbol{X})$ is optimal if and only if its marginals $\gamma_{i}$ are absolutely continuous w.r.t. $\mu_{i}$,

$$
d \geq \pi / 2 \text { in }\left(\operatorname{supp} \mu_{1}^{\perp} \times \operatorname{supp} \mu_{2}\right) \bigcup\left(\operatorname{supp} \mu_{1} \times \operatorname{supp} \mu_{2}^{\perp}\right),
$$

and there exist Borel sets $A_{i} \subset \operatorname{supp} \gamma_{i}$ with $\gamma_{i}\left(X \backslash A_{i}\right)=0$ and Borel densities $\sigma_{i}: A_{i} \rightarrow(0, \infty)$ of $\gamma_{i}$ w.r.t. $\mu_{i}$ such that

$$
\begin{array}{ll}
\sigma_{1}\left(x_{1}\right) \sigma_{2}\left(x_{2}\right) \geq \cos ^{2}\left(\mathrm{~d}_{\pi / 2}\left(x_{1}, x_{2}\right)\right) & \text { in } A_{1} \times A_{2}, \\
\sigma_{1}\left(x_{1}\right) \sigma_{2}\left(x_{2}\right)=\cos ^{2}\left(\mathrm{~d}_{\pi / 2}\left(x_{1}, x_{2}\right)\right) & \boldsymbol{\gamma} \text {-a.e. in } A_{1} \times A_{2},
\end{array}
$$

or, equivalently, in terms of the densities $\varrho_{i}=\sigma_{i}^{-1}$ of $\mu_{i}$ w.r.t. $\gamma_{i}$

$$
\begin{aligned}
& \varrho_{1}\left(x_{1}\right) \varrho_{2}\left(x_{2}\right) \cos ^{2}\left(\mathrm{~d}_{\pi / 2}\left(x_{1}, x_{2}\right)\right) \leq 1 \quad \text { in } A_{1} \times A_{2}, \\
& \varrho_{1}\left(x_{1}\right) \varrho_{2}\left(x_{2}\right) \cos ^{2}\left(\mathrm{~d}_{\pi / 2}\left(x_{1}, x_{2}\right)\right)=1 \quad \boldsymbol{\gamma} \text {-a.e. in } A_{1} \times A_{2} .
\end{aligned}
$$

(c) $\ell(\mathrm{d})$-cyclical monotonicity. Every optimal plan $\gamma \in \mathrm{Opt}_{\mathbb{E T}}\left(\mu_{1}, \mu_{2}\right)$ is a solution of the optimal transport problem $\mathrm{T}$ with cost $\ell(\mathrm{d})$ (see (3.15) of 
Sect.3.3) between its marginals $\gamma_{i}$. In particular it is $\ell(\mathrm{d})$-cyclically monotone, i.e. it is concentrated on a Borel set $G \subset X(G=\operatorname{supp}(\gamma)$ when $\mathrm{d}$ is continuous) such that for every choice of $\left(x_{1}^{n}, x_{2}^{n}\right)_{n=1}^{N} \subset G$ and every permutation $\kappa:\{1, \ldots, N\} \rightarrow\{1, \ldots, N\}$

$$
\Pi_{n=1}^{N} \cos ^{2}\left(\mathrm{~d}_{\pi / 2}\left(x_{1}^{n}, x_{2}^{n}\right)\right) \geq \Pi_{n=1}^{N} \cos ^{2}\left(\mathrm{~d}_{\pi / 2}\left(x_{1}^{n}, x_{2}^{\kappa(n)}\right)\right) .
$$

(d) Generalized potentials. If $\gamma$ is optimal and $A_{i}, \sigma_{i}$ are defined as in $b$ ) above, the Borel potentials $\varphi_{i}, \psi_{i}: X \rightarrow \overline{\mathbb{R}}$

$$
\varphi_{i}:=\left\{\begin{array}{ll}
-\log \sigma_{i} & \text { in } A_{i}, \\
-\infty & \text { in } X \backslash \operatorname{supp} \mu_{i}, \\
+\infty & \text { otherwise, }
\end{array} \quad \psi_{i}:= \begin{cases}1-\sigma_{i} & \text { in } A_{i}, \\
-\infty & \text { in } X \backslash \operatorname{supp} \mu_{i}, \\
1 & \text { otherwise, }\end{cases}\right.
$$

satisfy $\varphi_{1} \oplus_{o} \varphi_{2} \leq \ell(\mathrm{d}), \log \left(1-\psi_{1}\right) \oplus_{o} \log \left(1-\psi_{2}\right) \geq \ell(\mathrm{d})$, and the optimality conditions corresponding to (4.50)

$$
\begin{aligned}
\varphi_{1}\left(x_{1}\right)+\varphi_{2}\left(x_{2}\right) & =\log \left(1-\psi_{1}\left(x_{1}\right)\right)+\log \left(1-\psi_{2}\left(x_{2}\right)\right) \\
& =\ell\left(\mathrm{d}\left(x_{1}, x_{2}\right)\right) \quad \gamma \text {-a.e. in } A_{1} \times A_{2} .
\end{aligned}
$$

Moreover $\mathrm{e}^{-\varphi_{i}}, \psi_{i} \in \mathrm{L}^{1}\left(X, \mu_{i}\right)$ and

$$
\begin{aligned}
\operatorname{LET}\left(\mu_{1}, \mu_{2}\right) & =\sum_{i} \int_{X}\left(1-\mathrm{e}^{-\varphi_{i}}\right) \mathrm{d} \mu_{i} \\
& =\sum_{i} \int_{X} \psi_{i} \mathrm{~d} \mu_{i}=\sum_{i} \mu_{i}(X)-2 \gamma(X) .
\end{aligned}
$$

Proof Identity (6.13) follows by Theorem 4.11, recalling the definition (4.11) of $\boldsymbol{\Phi}$ and the fact that $F_{i}^{\circ}(\varphi)=1-\exp (-\varphi)$.

Identity (6.14) follows from Proposition 4.3 and the fact that $R_{i}^{*}(\psi)=$ $-\log (1-\psi)$. Notice that the definition (4.7) of $\boldsymbol{\Psi}$ ensures that we can restrict the supremum in (6.14) to functions $\psi_{i}$ with $\sup _{X} \psi_{i}<1$. We have discussed the possibility to replace $\operatorname{LSC}_{s}(X)$ with $\operatorname{LSC}_{b}(X), \mathrm{B}_{b}(X)$ or $\mathrm{C}_{b}(X)$ in Corollary 4.12.

The statement of point (b) follows by Corollary 4.18; notice that the LET problem is always feasible.

Point (c) is an obvious consequence of the optimality of $\gamma$.

Point (d) can be easily deduced by (b) or by applying Corollaries 4.17 and 4.18, observing that the formula defining $\varphi_{i}$ of (6.21) corresponds to (4.48) with $\left(F_{i}\right)_{\infty}^{\prime}=+\infty=-\left(F_{i}\right)_{0}^{\prime}$ and the optimality condition corresponds to (4.50). Finally, $\psi_{i}$ are just related to $\varphi_{i}$ by $\psi_{i}=F_{i}^{\circ}\left(\varphi_{i}\right)=1-\exp \left(-\varphi_{i}\right)$. 
In the one-dimensional case, the $\ell(d)$-cyclic monotonicity of part (c) of the previous theorem reduces to classical monotonicity.

Corollary 6.4 (Monotonicity of optimal plans in $\mathbb{R}$ ) When $X=\mathbb{R}$ with the usual distance, the support of every optimal plan $\gamma$ is a monotone set, i.e.

$$
\left(x_{1}, x_{2}\right),\left(x_{1}^{\prime}, x_{2}^{\prime}\right) \in \operatorname{supp}(\boldsymbol{\gamma}), x_{1}<x_{1}^{\prime} \Rightarrow x_{2} \leq x_{2}^{\prime} .
$$

Proof As the function $\ell$ is uniformly convex, (6.20) is equivalent to monotonicity.

The next result provides a variant of the reverse formulation in Theorem 6.2, which expresses the LET problem as a supremum of the linear mass functional on $\boldsymbol{\gamma}$ on a convex set characterized by the marginals of $\gamma$ and the cost.

Corollary 6.5 For all $\mu_{1}, \mu_{2} \in \mathcal{M}(X)$ we have

$$
\begin{aligned}
\text { LET }\left(\mu_{1}, \mu_{2}\right)= & \sum_{i} \mu_{i}(X)-2 \max \left\{\boldsymbol{\gamma}(\boldsymbol{X}): \boldsymbol{\gamma} \in \mathcal{M}(\boldsymbol{X}), \gamma_{i}=\sigma_{i} \mu_{i},\right. \\
& \left.\sigma_{1}\left(x_{1}\right) \sigma_{2}\left(x_{2}\right) \leq \cos ^{2}\left(\mathrm{~d}_{\pi / 2}\left(x_{1}, x_{2}\right)\right) \boldsymbol{\gamma} \text {-a.e. in } \boldsymbol{X}\right\} .
\end{aligned}
$$

Proof Let us denote by $M^{\prime}$ the right-hand side and let $\boldsymbol{\gamma} \in \mathcal{M}(X)$ be a plan satisfying the conditions of (6.24). If $A_{i}$ are Borel sets with $\gamma_{i}\left(X \backslash A_{i}\right)=0$ and $\sigma_{i}$ : $X \rightarrow(0, \infty)$ are Borel densities of $\gamma_{i}$ w.r.t. $\mu_{i}$, the densities $\varrho_{i}$ of $\mu_{i}$ w.r.t. $\gamma_{i}$ satisfy $\varrho_{i}\left(x_{i}\right)=1 / \sigma_{i}\left(x_{i}\right)$ in $A_{i}$ so that $\sigma_{1}\left(x_{1}\right) \sigma_{2}\left(x_{2}\right) \leq \cos ^{2}\left(\mathrm{~d}_{\pi / 2}\left(x_{1}, x_{2}\right)\right)$ yields $\varrho_{1}\left(x_{1}\right) \varrho_{2}\left(x_{2}\right) \cos ^{2}\left(\mathrm{~d}_{\pi / 2}\left(x_{1}, x_{2}\right)\right) \geq 1$. Since $\left(\log \varrho_{i}\right)_{+} \in \mathrm{L}^{1}\left(X, \gamma_{i}\right)$ we have

$$
\begin{aligned}
& \sum_{i}\left(\mu_{i}^{\perp}(X)+\int_{X}\left(\varrho_{i}-1-\log \varrho_{i}\right) \mathrm{d} \gamma_{i}\right)+\int_{X} \ell\left(\mathrm{d}\left(x_{1}, x_{2}\right)\right) \mathrm{d} \boldsymbol{\gamma} \\
& =\sum_{i}\left(\mu_{i}(X)-\gamma_{i}(X)\right)-\int_{X} \log \left(\varrho_{1}\left(x_{1}\right) \varrho_{2}\left(x_{2}\right) \cos ^{2}\left(\mathrm{~d}_{\pi / 2}\left(x_{1}, x_{2}\right)\right)\right) \mathrm{d} \boldsymbol{\gamma} \\
& \quad \leq \sum_{i} \mu_{i}(X)-2 \boldsymbol{\gamma}(X) .
\end{aligned}
$$

By (6.11) we get $M^{\prime} \geq \operatorname{LT}\left(\mu_{1}, \mu_{2}\right)$. On the other hand, choosing any $\bar{\gamma} \in$ $\mathrm{Opt}_{\text {EFT }}\left(\mu_{1}, \mu_{2}\right)$ the optimality condition (6.17) shows that $\bar{\gamma}$ is an admissible competitor for (6.24) and (6.22) shows that $M^{\prime}=\operatorname{LET}\left(\mu_{1}, \mu_{2}\right)$.

Combining (6.12), (6.13), (6.14), and (6.24), we find that the nonnegative and concave functional $\left(\mu_{1}, \mu_{2}\right) \mapsto \sum_{i} \mu_{i}(X)-\mathbb{E} T\left(\mu_{1}, \mu_{2}\right)$ can be represented as in the following equivalent ways: 


$$
\begin{aligned}
& \sum_{i} \mu_{i}(X)-\mathbf{E T}\left(\mu_{1}, \mu_{2}\right)=2 \max _{\boldsymbol{\gamma} \in \mathcal{M}(\boldsymbol{X})} \int_{\boldsymbol{X}} \sqrt{\varrho_{1}\left(x_{1}\right) \varrho_{2}\left(x_{2}\right)} \cos \left(\mathrm{d}_{\pi / 2}\left(x_{1}, x_{2}\right)\right) \mathrm{d} \boldsymbol{\gamma} \\
& =\inf \left\{\sum_{i} \int_{X} \mathrm{e}^{-\varphi_{i}} \mathrm{~d} \mu_{i}: \varphi_{i} \in \operatorname{LSC}_{S}(X), \varphi_{1} \oplus \varphi_{2} \leq \ell(\mathrm{d})\right\} \\
& =\inf \left\{\sum_{i} \int_{X} \tilde{\psi}_{i} \mathrm{~d} \mu_{i}: \tilde{\psi}_{i} \in \operatorname{USC}_{s}(X), \inf _{X} \tilde{\psi}_{i}>0\right. \\
& =2 \max \left\{\boldsymbol{\gamma}(\boldsymbol{X}): \boldsymbol{\gamma} \in \mathcal{M}(\boldsymbol{X}), \gamma_{i}=\sigma_{i} \mu_{i},\right. \\
& \left.\sigma_{1}\left(x_{1}\right) \sigma_{2}\left(x_{2}\right) \leq \cos ^{2}\left(\mathrm{~d}_{\pi / 2}\left(x_{1}, x_{2}\right)\right) \boldsymbol{\gamma} \text {-a.e. in } \boldsymbol{X}\right\}
\end{aligned}
$$

The next result concerns uniqueness of the optimal plan $\gamma$ in the Euclidean case $X=\mathbb{R}^{d}$. We will use the notion of approximate differential (denoted by D), see e.g. [2, Def. 5.5.1].

Theorem 6.6 (Uniqueness) Let $\mu_{i} \in \mathcal{M}(X)$ and $\gamma \in \mathrm{Opt}_{\text {LET }}\left(\mu_{1}, \mu_{2}\right)$.

(i) The marginals $\gamma_{i}=\pi_{\sharp}^{i} \gamma$ are uniquely determined.

(ii) If $X=\mathbb{R}$ with the usual distance then $\gamma$ is the unique element of $\mathrm{Opt}_{\mathbb{E T}}\left(\mu_{1}, \mu_{2}\right)$.

(iii) If $X=\mathbb{R}^{d}$ with the usual distance, $\mu_{1} \ll \mathscr{L}^{d}$ is absolutely continuous, and $A_{i} \subset \mathbb{R}^{d}$ and $\sigma_{i}: A_{i} \rightarrow(0, \infty)$ are as in Theorem $\left.6.3 \mathrm{~b}\right)$, then $\sigma_{1}$ is approximately differentiable at $\mu_{1}$-a.e. point of $A_{1}$ and $\gamma$ is the unique element of $\mathrm{Opt}_{\mathbb{E}}\left(\mu_{1}, \mu_{2}\right)$. The transport plan $\gamma$ is concentrated on the graph of a function $\boldsymbol{t}: \mathbb{R}^{d} \rightarrow \mathbb{R}^{d}$ satisfying

$$
\begin{aligned}
& \boldsymbol{t}\left(x_{1}\right)=x_{1}-\frac{\arctan \left(\left|\boldsymbol{\xi}\left(x_{1}\right)\right|\right)}{\left|\boldsymbol{\xi}\left(x_{1}\right)\right|} \boldsymbol{\xi}\left(x_{1}\right), \\
& \boldsymbol{\xi}\left(x_{1}\right)=-\frac{1}{2} \tilde{D} \log \sigma_{1}\left(x_{1}\right)
\end{aligned}
$$

Proof (i) follows directly from Lemma 3.5.

(ii) follows by Theorem 6.3(c), since whenever the marginals $\gamma_{i}$ are fixed there is only one plan with monotone support in $\mathbb{R}$ (see e.g. [42, Chap. 2]).

In order to prove (iii) we adapt the argument of [2, Thm. 6.2.4] to our singular setting, where the cost $\mathrm{c}$ can take the value $+\infty$.

Let $A_{i} \subset \mathbb{R}^{d}$ and $\sigma_{i}: A_{i} \rightarrow(0, \infty)$ as in Theorem $\left.6.3 \mathrm{~b}\right)$; notice that since $\sigma_{1}>0$ in $A_{1}$ the classes of $\mu_{1}$ - and $\gamma_{1}$-negligible subsets of $A_{1}$ coincide. 
Since $\mu_{1}=u \mathscr{L}^{d} \ll \mathscr{L}^{d}$ with density $u \in L^{1}\left(\mathbb{R}^{d}\right)$, up to removing a $\mu_{1^{-}}$ negligible set (and thus $\gamma_{1}$-negligible) from $A_{1}$, it is not restrictive to assume that $u\left(x_{1}\right)>0$ everywhere in $A_{1}$, so that the classes of $\mathscr{L}^{d}$ - and $\mu_{1}$-negligible subsets of $A_{1}$ coincide. For every $n \in \mathbb{N}$ we define

$$
A_{2, n}:=\left\{x_{2} \in A_{2}: \sigma_{2}\left(x_{2}\right) \geq 1 / n\right\}, \quad s_{n}\left(x_{1}\right):=\sup _{x_{2} \in A_{2, n}} \cos ^{2}\left(\left|x_{1}-x_{2}\right|\right) / \sigma_{2}\left(x_{2}\right) .
$$

The functions $s_{n}$ are bounded and Lipschitz in $\mathbb{R}^{d}$ and therefore differentiable $\mathscr{L}^{d}$-a.e. by Rademacher's Theorem. Since $\mu_{1}$ is absolutely continuous w.r.t. $\mathscr{L}^{d}$ we deduce that $s_{n}$ are differentiable $\mu_{1}$-a.e. in $A_{1}$.

By (6.16) we have $\sigma_{1}\left(x_{1}\right) \geq s_{n}\left(x_{1}\right)$ in $A_{1}$. By (6.17) we know that for $\gamma_{1^{-}}$ a.e. $x_{1} \in A_{1}$ there exists $x_{2} \in A_{2}$ such that $\left|x_{1}-x_{2}\right|<\pi / 2$ and $\sigma_{1}\left(x_{1}\right)=$ $\cos ^{2}\left(\left|x_{1}-x_{2}\right|\right) / \sigma_{2}\left(x_{2}\right)$ so that $\sigma_{1}\left(x_{1}\right)=s_{n}\left(x_{1}\right)$ for $n$ sufficiently big and hence the family $\left(B_{n}\right)_{n \in \mathbb{N}}$ of sets $B_{n}:=\left\{x_{1} \in A_{1}: \sigma_{1}\left(x_{1}\right)>s_{n}\left(x_{1}\right)\right\}$ is decreasing (since $s_{n}$ is increasing and dominated by $\sigma_{1}$ ) and has $\mathscr{L}^{d}$-negligible intersection.

It follows that $\gamma_{1}$-a.e. $x_{1} \in A_{1}$ is a point of $\mathscr{L}^{d}$-density 1 of $\left\{x_{1} \in A_{1}\right.$ : $\left.\sigma_{1}\left(x_{1}\right)=s_{n}\left(x_{1}\right)\right\}$ for some $n \in \mathbb{N}$ and $s_{n}$ is differentiable at $x_{1}$. Let us denote by $A_{1}^{\prime}$ the set of all $x_{1} \in A_{1}$ such that $\sigma_{1}$ is approximately differentiable at every $x_{1} \in A_{1}^{\prime}$ with approximate differential $\tilde{\mathrm{D}} \sigma_{1}\left(x_{1}\right)$ equal to $\mathrm{D} s_{n}\left(x_{1}\right)$ for $n$ sufficiently big.

Suppose now that $x_{1} \in A_{1}^{\prime}$ and $\sigma_{1}\left(x_{1}\right)=\cos ^{2}\left(\left|x_{1}-x_{2}\right|\right) / \sigma_{2}\left(x_{2}\right)$ for some $x_{2} \in A_{2}$. Since by (6.16) and (6.17) the map $x_{1}^{\prime} \mapsto \cos ^{2}\left(\left|x_{1}^{\prime}-x_{2}\right|\right) / \sigma_{1}\left(x_{1}^{\prime}\right)$ attains its maximum at $x_{1}^{\prime}=x_{1}$, we deduce that

$$
\tan \left(\left|x_{1}-x_{2}\right|\right) \frac{x_{1}-x_{2}}{\left|x_{1}-x_{2}\right|}=-\frac{1}{2} \tilde{D} \log \sigma_{1}\left(x_{1}\right),
$$

so that $x_{2}$ is uniquely determined, and (6.29) follows.

We conclude this section with the last representation formula for $\operatorname{LT}\left(\mu_{1}, \mu_{2}\right)$ given in terms of transport plans $\boldsymbol{\alpha}$ in $\boldsymbol{Y}:=Y \times Y$ with $Y:=X \times[0, \infty)$ with constraints on the homogeneous marginals, keeping the notation of Sect. 5.2. Even if it seems the most complicated one, it will provide the natural point of view in order to study the metric properties of the LFT functional, and it will play a crucial role in Sect. 7.6, where the link between the LET formulation and the Hellinger-Kantorovich distance will be studied. The interest of (6.34) relies in the particular form of its integrand, by recalling that by (5.4) and (6.9) we have

$$
\text { LET }\left(r_{1}^{2} \delta_{x_{1}}, r_{2}^{2} \delta\left(x_{2}\right)\right)=r_{1}^{2}+r_{2}^{2}-2 r_{1} r_{2} \cos \left(\mathrm{d}_{\pi / 2}\left(x_{1}, x_{2}\right)\right) .
$$


Theorem 6.7 For every $\mu_{i} \in \mathcal{M}(X)$ we have

$$
\begin{gathered}
\text { LET }\left(\mu_{1}, \mu_{2}\right)=\sum_{i} \mu_{i}(X)-2 \max _{\boldsymbol{\alpha} \in \mathcal{H}_{\leq}^{2}\left(\mu_{1}, \mu_{2}\right)} \int_{\boldsymbol{X}} r_{1} r_{2} \cos \left(\mathrm{d}_{\pi / 2}\left(x_{1}, x_{2}\right)\right) \mathrm{d} \boldsymbol{\alpha} \\
=\min \left\{\int_{\boldsymbol{Y}}\left(r_{1}^{2}+r_{2}^{2}-2 r_{1} r_{2} \cos \left(\mathrm{d}_{\pi / 2}\left(x_{1}, x_{2}\right)\right)\right) \mathrm{d} \boldsymbol{\alpha}\right. \\
\left.\quad+\sum_{i}\left(\mu_{i}-\mathrm{h}_{i}^{2}(\boldsymbol{\alpha})\right)(X): \boldsymbol{\alpha} \in \mathcal{M}(\boldsymbol{Y}), \mathrm{h}_{i}^{2}(\boldsymbol{\alpha}) \leq \mu_{i}\right\} \\
=\min \left\{\int_{\boldsymbol{Y}}\left(r_{1}^{2}+r_{2}^{2}-2 r_{1} r_{2} \cos \left(\mathrm{d}_{\pi / 2}\left(x_{1}, x_{2}\right)\right)\right) \mathrm{d} \boldsymbol{\alpha}: \boldsymbol{\alpha} \in \mathcal{M}(\boldsymbol{Y}), \mathrm{h}_{i}^{2}(\boldsymbol{\alpha})=\mu_{i}\right\}
\end{gathered}
$$

Moreover, for every plan $\bar{\gamma} \in \mathrm{Opt}_{\mathbb{E T}}\left(\mu_{1}, \mu_{2}\right)$ and every pair of Borel densities $\varrho_{i}$ as in (6.11) the plan $\bar{\alpha}:=\left(x_{1}, \sqrt{\varrho_{1}\left(x_{1}\right)} ; x_{2}, \sqrt{\varrho_{2}\left(x_{2}\right)}\right)_{\sharp} \bar{\gamma}$ is optimal for (6.33) and (6.32).

Proof Identity (6.33) (resp. (6.34)) follows directly by (5.32) (resp. (5.33)) of Theorem 5.8. Relation (6.32) is just a different form for (6.33).

\section{The metric side of the LET-functional: the Hellinger-Kantorovich distance}

In this section we want to show that the functional

$$
\left(\mu_{1}, \mu_{2}\right) \mapsto \sqrt{\mathrm{ETT}\left(\mu_{1}, \mu_{2}\right)}
$$

defines a distance in $\mathcal{M}(X)$, which is then called the Hellinger-Kantorovich distance and denoted $\mathrm{HK}$.

In order to introduce this distance we will adopt a geometric point of view, which is strictly related to the characterization given in Theorem 6.7: it will mainly exploit the link with Optimal Transport in the so-called geometric cone $\mathfrak{C}$ constructed on $X$, cf. [10, Sect. 3.6]. This is possible since the function

$$
\left(x_{1}, r_{1} ; x_{2}, r_{2}\right) \mapsto r_{1}^{2}+r_{2}^{2}-2 r_{1} r_{2} \cos \left(\mathrm{d}\left(x_{1}, x_{2}\right) \wedge a\right), \quad a>0,
$$

appearing in (6.31) and (6.34) with $a=\pi / 2$, is a (possibly extended) squared semidistance in $Y=X \times[0, \infty)$, whenever $a \in(0, \pi]$.

In the next two sections we will briefly study this function and the associated metric for the particular choices of $a=\pi$ (the canonical one in metric geometry) and $a=\pi / 2$ (related to the minimal cost between a pair of Dirac masses (6.31)): the role of these two values will be clarified in Remark 7.2 and in Sect. 7.6, we also refer to [30, Sect. 3] for more motivation and examples. The 
induced metric space $\mathfrak{C}$, can be obtained by taking the quotient with respect to the equivalence classes of points with distance 0 . Radon measures on $\mathfrak{C}$ can be projected to Radon measures on $X$ by taking suitable homogeneous marginals, which will be studied in Sect. 7.2.

The definition and the basic properties of the Hellinger-Kantorovich distance will be given in Sect. 7.3; the main metric properties will be derived in Sects. 7.4 and 7.5: they rely on a refined gluing technique and on the flexibility of the notion of homogeneous marginals, which allow us to transfer many useful properties of the Kantorovich-Wasserstein distance on the cone $\mathfrak{C}$ to corresponding properties for $\mathrm{HK}$. Section 7.6 will then show the equivalent characterization of $\mathrm{HK}$ in terms of the Logarithmic Entropy Transport problem LFT and its dual formulation, thus providing a direct and robust formulation of $\mathrm{HK}$ as a convex minimization problem enjoying all the properties we recalled in the previous section.

\subsection{The cone construction}

Let us quickly recall a few basic facts concerning the cone construction, referring to $[10$, Sect. 3.6] for further details. In the extended metric-topological space $(X, \tau, \mathrm{d})$ of Sect. 6.1 , we will denote by $\mathrm{d}_{a}:=\mathrm{d} \wedge a$ the truncated distance and by $y=(x, r), x \in X, r \in[0, \infty)$, the generic points of $Y:=X \times[0, \infty)$.

It is not difficult to show that the function $\mathrm{d}_{\mathfrak{C}}: Y \times Y \rightarrow[0, \infty)$

$$
\mathrm{d}_{\mathfrak{C}^{\mathrm{C}}}^{2}\left(\left(x_{1}, r_{1}\right),\left(x_{2}, r_{2}\right)\right):=r_{1}^{2}+r_{2}^{2}-2 r_{1} r_{2} \cos \left(\mathrm{d}_{\pi}\left(x_{1}, x_{2}\right)\right)
$$

is nonnegative, symmetric, and satisfies the triangle inequality (see e.g. [10, Prop. 3.6.13]). We also notice that

$$
\mathrm{d}_{\mathfrak{C}^{\mathrm{C}}}^{2}\left(y_{1}, y_{2}\right)=\left|r_{1}-r_{2}\right|^{2}+4 r_{1} r_{2} \sin ^{2}\left(\mathrm{~d}_{\pi}\left(x_{1}, x_{2}\right) / 2\right),
$$

which implies the useful estimates

$$
\begin{gathered}
\max \left(\left|r_{1}-r_{2}\right|, \frac{2}{\pi} \sqrt{r_{1} r_{2}} \mathrm{~d}_{\pi}\left(x_{1}, x_{2}\right)\right) \leq \mathrm{d}_{\mathfrak{C}}\left(y_{1}, y_{2}\right) \\
\leq\left|r_{1}-r_{2}\right|+\sqrt{r_{1} r_{2}} \mathrm{~d}_{\pi}\left(x_{1}, x_{2}\right) .
\end{gathered}
$$

From this it follows that $d_{\mathfrak{C}}$ induces a true distance in the quotient space $\mathfrak{C}=Y / \sim$ where

$$
y_{1} \sim y_{2} \quad \Leftrightarrow \quad r_{1}=r_{2}=0 \text { or } r_{1}=r_{2}, x_{1}=x_{2} .
$$


Equivalence classes are usually denoted by $\mathfrak{y}=[y]=[x, r]$, where the vertex $[x, 0]$ plays a distinguished role. It is denoted by $\mathfrak{o}$, its complement is the open set $\mathfrak{C}_{\mathfrak{o}}=\mathfrak{C} \backslash\{\mathfrak{o}\}$. On $\mathfrak{C}$ we introduce a topology $\tau_{\mathfrak{C}}$, which is in general weaker than the canonical quotient topology: $\tau_{\mathfrak{C}}$ neighborhoods of points in $\mathfrak{C}_{\mathfrak{o}}$ coincide with neighborhoods in $Y$, whereas the sets

$$
\{[x, r]: 0 \leq r<\varepsilon\}=\left\{\mathfrak{y} \in \mathfrak{C}: \mathrm{d}_{\mathfrak{C}}(\mathfrak{y}, \mathfrak{o})<\varepsilon\right\}, \quad \varepsilon>0,
$$

provide a system of open neighborhoods of $\mathfrak{o}$. $\tau_{\mathfrak{C}}$ coincides with the quotient topology when $X$ is compact.

It is easy to check that $\left(\mathfrak{C}, \tau_{\mathfrak{C}}\right)$ is a Hausdorff topological space and $d_{\mathfrak{C}}$ is $\tau_{\mathfrak{C}}-$ lower semicontinuous. If $\tau$ is induced by $\mathrm{d}$ then $\tau_{\mathfrak{C}}$ is induced by $\mathrm{d}_{\mathfrak{C}}$. If $(X, \mathrm{~d})$ is complete (resp. separable), then $\left(\mathfrak{C}, d_{\mathfrak{C}}\right)$ is also complete (resp. separable).

Perhaps the simplest example is provided by the unit sphere $X=\mathbb{S}^{d-1}=$ $\left\{x \in \mathbb{R}^{d}:|x|=1\right\}$ in $\mathbb{R}^{d}$ endowed with the intrinsic Riemannian distance: the corresponding cone $\mathfrak{C}$ is precisely $\mathbb{R}^{d}$.

We denote the canonical projection by

$$
\mathfrak{p}: Y \rightarrow \mathfrak{C}, \quad \mathfrak{p}(x, r)=[x, r] .
$$

Clearly $\mathfrak{p}$ is continuous and is an homeomorphism between $Y \backslash(X \times\{0\})$ and $\mathfrak{C}_{\mathfrak{o}}$. A right inverse $\mathrm{y}: \mathfrak{C} \rightarrow Y$ of the map $\mathfrak{p}$ can be obtained by fixing a point $\bar{x} \in X$ and defining

$$
\begin{aligned}
& \mathrm{r}: \mathfrak{C} \rightarrow[0, \infty), \mathrm{r}[x, r]=r, \\
& \mathrm{x}: \mathfrak{C} \rightarrow X, \mathrm{x}[x, r]=\left\{\begin{array}{ll}
x & \text { if } r>0, \\
\bar{x} & \text { if } r=0,
\end{array} \text { and } \mathrm{y}:=(\mathrm{x}, \mathrm{r}) .\right.
\end{aligned}
$$

Notice that $r$ is continuous and $x$ is continuous restricted to $\mathfrak{C}_{\mathfrak{o}}$.

A continuous rescaling product from $\mathfrak{C} \times[0, \infty)$ to $\mathfrak{C}$ can be defined by

$$
\mathfrak{y} \cdot \lambda:= \begin{cases}\mathfrak{o} & \text { if } \mathfrak{y}=\mathfrak{o}, \\ {[x, \lambda r]} & \text { if } \mathfrak{y}=[x, r], s>0 .\end{cases}
$$

We conclude this introductory section by a characterization of compact sets in $\left(\mathfrak{C}, \tau_{\mathfrak{C}}\right)$.

Lemma 7.1 (Compact sets in $\mathfrak{C}$ ) A closed set $K$ of $\mathfrak{C}$ is compact if and only if there is $r_{0}>0$ such that its upper sections

$$
K(\rho):=\{x \in X:[x, r] \in K \text { for some } r \geq \rho\}
$$

are empty for $\rho>r_{0}$ and compact in $X$ for $0<\rho \leq r_{0}$. 
Proof It is easy to check that the condition is necessary.

In order to show the sufficiency, let $\rho=\inf _{K}$ r. If $\rho>0$ then $K$ is compact since it is a closed subset of the compact set $\mathfrak{p}\left(K(\rho) \times\left[\rho, r_{0}\right]\right)$.

If $\rho=0$ then $\mathfrak{o}$ is an accumulation point of $K$ by (7.7) and therefore $\mathfrak{o} \in K$ since $K$ is closed. If $\mathscr{U}$ is an open covering of $K$, we can pick $U_{0} \in \mathscr{U}$ such that $\mathfrak{o} \in U_{0}$. By (7.7) there exists $\varepsilon>0$ such that $K \backslash U_{0} \subset \mathfrak{p}(K(\varepsilon) \times$ $\left.\left[\varepsilon, r_{0}\right]\right)$ : since $\mathfrak{p}\left(K(\varepsilon) \times\left[\varepsilon, r_{0}\right]\right)$ is compact, we can thus find a finite subcover $\left\{U_{1}, \cdots, U_{N}\right\} \subset \mathscr{U}$ of $K \backslash U_{0} .\left\{U_{n}\right\}_{n=0}^{N}$ is therefore a finite subcover of $K$.

Remark 7.2 (Two different truncations) Notice that in the constitutive formula defining $\mathrm{d}_{\mathfrak{C}}$ we used the truncated distance $\mathrm{d}_{\pi}$ with upper threshold $\pi$, whereas in Theorem 6.7 an analogous formula with $\mathrm{d}_{\pi / 2}$ and threshold $\pi / 2$ played a crucial role. We could then consider the distance

$$
\begin{aligned}
& \mathrm{d}_{\pi / 2, \mathfrak{C}}^{2}\left(\left[x_{1}, r_{1}\right],\left[x_{2}, r_{2}\right]\right):=r_{1}^{2}+r_{2}^{2}-2 r_{1} r_{2} \cos \left(\mathrm{d}_{\pi / 2}\left(x_{1}, x_{2}\right)\right) \\
& \quad=\left|r_{1}-r_{2}\right|^{2}+4 r_{1} r_{2} \sin ^{2}\left(\mathrm{~d}_{\pi / 2}\left(x_{1}, x_{2}\right) / 2\right)
\end{aligned}
$$

on $\mathfrak{C}$, which satisfies

$$
\mathrm{d}_{\pi / 2, \mathfrak{C}} \leq \mathrm{d}_{\mathfrak{C}} \leq \sqrt{2} \mathrm{~d}_{\pi / 2, \mathfrak{C}}
$$

The notation (7.11a) is justified by the fact that $d_{\pi / 2, \mathfrak{C}}$ is still a cone distance associated to the metric space $\left(X, \mathrm{~d}_{\pi / 2}\right)$, since obviously $\left(\mathrm{d}_{\pi / 2}\right)_{\pi}=\left(\mathrm{d}_{\pi / 2}\right) \wedge$ $\pi / 2=d_{\pi / 2}$. From the geometric point of view, the choice of $d_{\mathfrak{C}}$ is natural, since it preserves important metric properties concerning geodesics (see [10, Thm. 3.6.17] and the next Sect. 8.1) and curvature (see [10, Sect. 4.7] and the next Sect. 8.3).

On the other hand, the choice of $\mathrm{d}_{\pi / 2}$ is crucial for its link with the function $H$ of (6.9), with Entropy-Transport problems, and with a representation property for the Hopf-Lax formula that we will see in the next sections. Notice that the 1-homogeneous formula (6.7) would not be convex in $\left(r_{1}, r_{2}\right)$ if one uses $\mathrm{d}_{\pi}$ instead of $\mathrm{d}_{\pi / 2}$. Nevertheless, we will prove in Sect. 7.3 the remarkable fact that both $d_{\pi}$ and $d_{\pi / 2}$ will lead to the same distance between positive measures.

\subsection{Radon measures in the cone $\mathfrak{C}$ and homogeneous marginals}

It is clear that any measure $v \in \mathcal{M}(\mathfrak{C})$ can be lifted to a measure $\bar{v} \in \mathcal{M}(Y)$ such that $\mathfrak{p}_{\sharp} \bar{v}=v$ : it is sufficient to take $\bar{v}=\mathrm{y}_{\sharp} v$ where $\mathrm{y}$ is a right inverse of $\mathfrak{p}$ defined as in (7.9). 
We call $\mathcal{M}_{2}(\mathfrak{C})\left(\right.$ resp. $\left.\mathcal{P}_{2}(\mathfrak{C})\right)$ the space of measures $v \in \mathcal{M}(\mathfrak{C})$ (resp. $v \in$ $\mathcal{P}(\mathfrak{C}))$ such that

$$
\int_{\mathfrak{C}} \mathrm{r}^{2} \mathrm{~d} v=\int_{\mathfrak{C}} \mathrm{d}_{\mathfrak{C}}^{2}(\mathfrak{y}, \mathfrak{o}) \mathrm{d} v=\int_{Y} r^{2} \mathrm{~d} \bar{v}<\infty, \quad \bar{v}=\mathrm{y}_{\sharp} \nu .
$$

Measures in $\mathcal{M}_{2}(\mathfrak{C})$ thus correspond to images $\mathfrak{p}_{\sharp} \bar{v}$ of measures $\bar{v} \in \mathcal{M}_{2}(Y)$ and have finite second moment w.r.t. the distance $\mathfrak{d}_{\mathfrak{C}}$, which justifies the index 2 in $\mathcal{M}_{2}(\mathfrak{C})$. Notice moreover that the measure $r^{2} \bar{v}$ does not charge $X \times\{0\}$ and it is independent of the choice of the point $\bar{x}$ in (7.9).

The above considerations can be easily extended to plans in the product spaces $\mathfrak{C}^{\otimes N}$ (where typically $N=2$, but also the general case will turn out to be useful later on). To clarify the notation, we will denote by $\mathfrak{y}=\left(\mathfrak{y}_{i}\right)_{i=1}^{N}=$ $\left(\left[x_{i}, r_{i}\right]\right)_{i=1}^{N}$ a point in $\mathfrak{C}^{\otimes N}$ and we will set $\mathbf{r}_{i}(\mathfrak{y})=\mathrm{r}\left(\mathfrak{y}_{i}\right)=r_{i}, \mathbf{x}_{i}(\mathfrak{y})=$ $\mathbf{X}\left(\mathfrak{y}_{i}\right) \in X$. Projections on the $i$-coordinate from $\mathfrak{C}^{\otimes N}$ to $\mathfrak{C}^{\mathfrak{s}}$ are usually denoted by $\pi^{i}$ or $\pi^{\mathfrak{y}_{i}}, \mathfrak{p}=\mathfrak{p}^{\otimes N}:(Y)^{\otimes N} \rightarrow \mathfrak{C}^{\otimes N}, \mathbf{y}=\mathrm{y}^{\otimes N}: \mathfrak{C}^{\otimes N} \rightarrow(Y)^{\otimes N}$ are the Cartesian products of the projections and of the lifts.

Recall that the $\mathrm{L}^{2}$-Kantorovich-Wasserstein (extended) distance $\mathrm{W}_{\mathrm{d}_{\mathfrak{C}}}$ in $\mathcal{M}_{2}(\mathfrak{C})$ induced by $\mathrm{d}_{\mathfrak{C}}$ is defined by

$$
\mathrm{W}_{\mathrm{d}_{\mathfrak{C}}}^{2}\left(v_{1}, v_{2}\right):=\min \left\{\int \mathrm{d}_{\mathfrak{C}}^{2}\left(\mathfrak{y}_{1}, \mathfrak{y}_{2}\right) \mathrm{d} \boldsymbol{\alpha}: \boldsymbol{\alpha} \in \mathcal{M}(\mathfrak{C}), \pi_{\sharp}^{\mathfrak{y}_{i}} \boldsymbol{\alpha}=v_{i}\right\},
$$

with the convention that $W_{\mathfrak{d}_{\mathfrak{C}}}\left(v_{1}, v_{2}\right)=+\infty$ if $v_{1}(\mathfrak{C}) \neq v_{2}(\mathfrak{C})$ and thus the minimum in (7.14) is taken on an empty set. We want to mimic the above definition, replacing the usual marginal conditions in (7.14) with the homogeneous marginals $\mathfrak{h}_{i}^{2}$ which we are going to define.

Let us consider now a plan $\boldsymbol{\alpha}$ in $\mathcal{M}\left(\mathfrak{C}^{\otimes N}\right)$ with $\overline{\boldsymbol{\alpha}}=\mathbf{y}_{\sharp} \boldsymbol{\alpha} \in \mathcal{M}\left(Y^{\otimes N}\right)$ : we say that $\boldsymbol{\alpha}$ lies in $\mathcal{M}_{2}\left(\mathfrak{C}^{\otimes N}\right)$ if

$$
\int_{\mathfrak{C}^{\otimes N}} \sum_{i} \mathrm{r}_{i}^{2} \mathrm{~d} \boldsymbol{\alpha}=\int_{Y \otimes N} \sum_{i} r_{i}^{2} \mathrm{~d} \overline{\boldsymbol{\alpha}}<\infty
$$

Its "canonical" marginals in $\mathcal{M}(\mathfrak{C})$ are $\boldsymbol{\alpha}_{i}=\pi_{\sharp}^{\mathfrak{y}_{i}} \boldsymbol{\alpha}$, whereas the "homogeneous" marginals correspond to (5.24) with $p=2$ :

$$
\mathfrak{h}_{i}^{2}(\boldsymbol{\alpha}):=\left(\mathrm{x}_{i}\right)_{\sharp}\left(\mathrm{r}_{i}^{2} \boldsymbol{\alpha}\right)=\pi_{\sharp}^{x_{i}}\left(r_{i}^{2} \overline{\boldsymbol{\alpha}}\right)=\mathrm{h}_{i}^{2}(\overline{\boldsymbol{\alpha}}) \in \mathcal{M}(X), \quad \overline{\boldsymbol{\alpha}}:=\mathbf{y}_{\sharp} \boldsymbol{\alpha} .
$$

We will omit the index $i$ when $N=1$. Notice that $r_{i}^{2} \alpha$ does not charge $\left(\pi^{i}\right)^{-1}(\mathfrak{o})$ (similarly, $r_{i}^{2} \overline{\boldsymbol{\alpha}}$ does not charge $Y^{\otimes i-1} \times\{(\bar{x}, 0)\} \times Y^{\otimes N-i}$ ) so that (7.16) is independent of the choice of the point $\bar{x}$ in (7.9). 
As in (5.26), the homogeneous marginals on the cone are invariant with respect to dilations: if $\vartheta: \mathfrak{C}^{\otimes N} \rightarrow(0, \infty)$ is a Borel map in $\mathrm{L}^{2}\left(\mathfrak{C}^{\otimes N}, \boldsymbol{\alpha}\right)$ we set

$$
\left(\operatorname{prd}_{\vartheta}(\mathfrak{y})\right)_{i}:=\mathfrak{y}_{i} \cdot(\vartheta(\mathfrak{y}))^{-1} \text { and } \operatorname{dil}_{\vartheta, 2}(\boldsymbol{\alpha}):=\left(\operatorname{prd}_{\vartheta}\right)_{\sharp}\left(\vartheta^{2} \boldsymbol{\alpha}\right),
$$

so that

$$
\mathfrak{h}_{i}^{2}\left(\operatorname{dil}_{\vartheta, 2}(\boldsymbol{\alpha})\right)=\mathfrak{h}_{i}^{2}(\boldsymbol{\alpha}) \text { for every } \boldsymbol{\alpha} \in \mathcal{M}_{2}\left(\mathfrak{C}^{\otimes N}\right) .
$$

As for the canonical marginals, a uniform control of the homogeneous marginals is sufficient to get equal tightness, cf. (2.4) for the definition. We state this result for an arbitrary number of components, and we emphasize that we are not claiming any closedness of the involved sets.

Lemma 7.3 (Homogeneous marginals and tightness) Let $\mathcal{K}_{i}, i=1, \cdots, N$, be a finite collection of bounded and equally tight sets in $\mathcal{M}(X)$. Then, the set

$$
\left\{\boldsymbol{\alpha} \in \mathcal{M}_{2}\left(\mathfrak{C}^{N}\right): \mathfrak{h}_{i}^{2} \boldsymbol{\alpha} \in \mathcal{K}_{i} \text { for } i=1, \ldots, N\right\}
$$

is equally tight in $\mathcal{M}\left(\mathfrak{C}^{N}\right)$.

Proof By applying [2, Lem. 5.2.2], it is sufficient to consider the case $N=1$ : given a bounded and equally tight set $\mathcal{K} \subset \mathcal{M}(X)$ we prove that $\mathcal{H}:=\{\alpha \in$ $\left.\mathcal{M}_{2}(\mathfrak{C}): \mathfrak{h}^{2} \boldsymbol{\alpha} \in \mathcal{K}\right\}$ is equally tight. For $A \subset X, R \subset(0, \infty)$ we will use the short notation $A \times \mathfrak{C} R$ for $\mathfrak{p}(A \times R) \subset \mathfrak{C}$. If $A$ and $R$ are compact, then $A \times \mathfrak{C} R$ is compact in $\mathfrak{C}$.

Let $M:=\sup _{\mu \in \mathcal{K}} \mu(X)<\infty$; since $\mathcal{K}$ is equally tight, we can find an increasing sequence of compact sets $K_{n} \subset X$ such that $\mu\left(X \backslash K_{n}\right) \leq 8^{-n}$ for every $\mu \in \mathcal{K}$. For an integer $m \in \mathbb{N}$ we then consider the compact sets $\mathfrak{K}_{m} \subset \mathfrak{C}$ defined by

$$
\mathfrak{K}_{m}=\{\mathfrak{o}\} \cup\left(K_{m} \times \mathfrak{C}\left[2^{-m}, 2^{m}\right]\right) \cup\left(\bigcup_{n=1}^{\infty} K_{n+m} \times \mathfrak{C}\left[2^{-n}, 2^{-n+1}\right]\right) .
$$

Setting $K_{\infty}=\bigcup_{n=1}^{\infty} K_{n}$, we have $\mu\left(X \backslash K_{\infty}\right)=0$ and

$$
\begin{aligned}
\mathfrak{C} \backslash \mathfrak{K}_{m} \subset & \left(K_{m} \times \mathfrak{C}\left(2^{m}, \infty\right)\right) \cup\left(\bigcup_{n=1}^{\infty}\left(K_{n+m} \backslash K_{n+m-1}\right) \times \mathfrak{C}\left(2^{-n+1}, \infty\right)\right) \\
& \cup\left(\left(X \backslash K_{\infty}\right) \times_{\mathfrak{C}}(0, \infty)\right) .
\end{aligned}
$$

Since for every $\boldsymbol{\alpha} \in \mathcal{H}$ with $\mathfrak{h}^{2} \boldsymbol{\alpha}=\mu$ and every $A \in \mathcal{B}(X)$ we have

$$
\boldsymbol{\alpha}\left(A \times_{\mathfrak{C}}(s, \infty)\right) \leq s^{-2} \mu(A) \leq s^{-2} M \text { and } \boldsymbol{\alpha}\left(\left(X \backslash K_{\infty}\right) \times_{\mathfrak{C}}(0, \infty)\right)=0,
$$


we conclude

$$
\begin{aligned}
\boldsymbol{\alpha}\left(\mathfrak{C} \backslash \mathfrak{K}_{m}\right) & \leq M 4^{-m}+\sum_{n=1}^{\infty} \boldsymbol{\alpha}\left(\left(X \backslash K_{n+m-1}\right) \times_{\mathfrak{C}}\left(2^{-n+1}, \infty\right)\right) \\
& \leq M 4^{-m}+\sum_{n=1}^{\infty} 4^{n-1} 8^{1-n-m} \leq 4^{-m}\left(M+\sum_{n=1}^{\infty} 4^{-n}\right) \\
& \left.\leq 4^{-m}(1+M)\right),
\end{aligned}
$$

for every $\boldsymbol{\alpha} \in \mathcal{H}$. Since all $\mathfrak{K}_{m}$ are compact, we obtain the desired equal tightness.

\subsection{The Hellinger-Kantorovich problem}

In this section we will always consider $N=2$, keeping the shorter notation $\boldsymbol{Y}=Y^{\otimes 2}$ and $\mathfrak{C}=\mathfrak{C}^{\otimes 2}$. As in (5.28), for every $\mu_{1}, \mu_{2} \in \mathcal{M}_{2}(X)$ we define the sets

$$
\begin{aligned}
& \mathfrak{H}_{\leq}^{2}\left(\mu_{1}, \mu_{2}\right):=\left\{\boldsymbol{\alpha} \in \mathcal{M}_{2}(\mathfrak{C}): \mathfrak{h}_{i}^{2} \boldsymbol{\alpha} \leq \mu_{i}\right\} \text { and } \\
& \mathfrak{H}_{=}^{2}\left(\mu_{1}, \mu_{2}\right):=\left\{\boldsymbol{\alpha} \in \mathcal{M}_{2}(\mathfrak{C}): \mathfrak{h}_{i}^{2} \boldsymbol{\alpha}=\mu_{i}\right\} .
\end{aligned}
$$

They are the images of $\mathcal{H}_{\leq}^{2}\left(\mu_{1}, \mu_{2}\right)$ and $\mathcal{H}_{=}^{2}\left(\mu_{1}, \mu_{2}\right)$ through the projections $\mathfrak{p}_{\sharp}$; in particular they always contain plans $\mathfrak{p}_{\sharp} \boldsymbol{\alpha}$, where $\boldsymbol{\alpha}$ is given by (5.29). The condition $\alpha \in \mathfrak{H}_{\leq}^{2}\left(\mu_{1}, \mu_{2}\right)$ is equivalent to ask that

$$
\int \mathrm{r}_{i}^{2} \varphi\left(\mathrm{x}_{i}\right) \mathrm{d} \boldsymbol{\alpha} \leq \int \varphi \mathrm{d} \mu_{i} \text { for every nonnegative } \varphi \in \mathrm{B}_{b}(X) .
$$

We can thus define the following minimum problem:

Problem 7.4 (The Hellinger-Kantorovich problem) Given $\mu_{1}, \mu_{2} \in \mathcal{M}(X)$ find an optimal plan $\boldsymbol{\alpha}_{\mathrm{opt}} \in \mathcal{H}_{=}^{2}\left(\mu_{1}, \mu_{2}\right) \subset \mathcal{M}_{2}(\mathfrak{C})$ solving the minimum problem

$$
\mathrm{HK}\left(\mu_{1}, \mu_{2}\right)^{2}:=\min \left\{\int \mathrm{d}_{\mathfrak{C}^{(}}^{2}\left(\mathfrak{y}_{1}, \mathfrak{y}_{2}\right) \mathrm{d} \boldsymbol{\alpha}: \boldsymbol{\alpha} \in \mathcal{M}_{2}(\mathfrak{C}), \mathfrak{h}_{i}^{2} \boldsymbol{\alpha}=\mu_{i}\right\} .
$$

We denote by $\operatorname{Opt}_{\mathbb{K}_{K}}\left(\mu_{1}, \mu_{2}\right) \subset \mathcal{N}(\mathfrak{C})$ the collection of all the optimal plans $\boldsymbol{\alpha}$ realizing the minimum in (7.23) and by $\mathrm{H}^{2}\left(\mu_{1}, \mu_{2}\right)$ the value of the minimum in (7.23) (whose existence is guaranteed by the next Theorem 7.6).

Remark 7.5 (Lifting of plans in $Y$ ) Since any plan $\alpha \in \mathcal{M}(\mathfrak{C})$ can be lifted to a plan $\overline{\boldsymbol{\alpha}}=\mathbf{y}_{\sharp} \boldsymbol{\alpha} \in \mathcal{P}(Y \times Y)$ such that $\mathfrak{p}_{\sharp} \overline{\boldsymbol{\alpha}}=\boldsymbol{\alpha}$ the previous problem 7.4 is also equivalent to find 


$$
\min \left\{\int \mathrm{d}_{\mathfrak{C}^{c}}^{2}\left(y_{1}, y_{2}\right) \mathrm{d} \overline{\boldsymbol{\alpha}}: \overline{\boldsymbol{\alpha}} \in \mathcal{M}(Y \times Y), \quad \mathrm{h}_{i}^{2}(\overline{\boldsymbol{\alpha}})=\mu_{i}\right\} .
$$

The advantage to work in the quotient space $\mathfrak{C}$ is to gain compactness, as the next Theorem 7.6 will show.

An importance feature of the cone distance and the homogeneous marginals is an invariance under rescaling, which can be done by the dilations from (7.17). Let us set

$$
\mathfrak{C}[R]:=\{[x, r] \in \mathfrak{C}: r \leq R\} \text { and } \mathfrak{C}[R]:=\mathfrak{C}[R] \times \mathfrak{C}[R] .
$$

It is not restrictive to solve the previous problem 7.4 by also assuming that $\alpha$ is a probability plan in $\mathcal{P}(\mathfrak{C})$ concentrated on $\mathfrak{C}[R]$ with $R^{2}=\sum_{i} \mu_{i}(X)$, i.e.

$$
\begin{aligned}
\mathrm{HK}^{2}\left(\mu_{1}, \mu_{2}\right) & =\min _{\boldsymbol{\alpha} \in C} \int \mathrm{d}_{\mathfrak{C}}^{2} \mathrm{~d} \boldsymbol{\alpha}, \\
\text { where } C & :=\left\{\boldsymbol{\alpha} \in \mathcal{P}(\mathfrak{C}): \mathfrak{h}_{i}^{2} \boldsymbol{\alpha}=\mu_{i}, \boldsymbol{\alpha}(\mathfrak{C} \backslash \mathfrak{C}[R])=0\right\} .
\end{aligned}
$$

In fact the functional $d_{\mathfrak{C}}^{2}$ and the constraints have a natural scaling invariance induced by the dilation maps defined by (7.17). Since

$$
\int \mathrm{d}_{\mathfrak{C}}^{2} \mathrm{~d}\left(\operatorname{dil}_{\vartheta, 2}(\boldsymbol{\alpha})\right)=\int \vartheta^{2} \mathrm{~d}_{\mathfrak{C}}^{2}\left(\left[x_{1}, r_{1} / \vartheta\right] ;\left[x_{2}, r_{2} / \vartheta\right]\right) \mathrm{d} \boldsymbol{\alpha}=\int \mathrm{d}_{\mathfrak{C}}^{2} \mathrm{~d} \boldsymbol{\alpha},
$$

restricting first $\boldsymbol{\alpha}$ to $\mathfrak{C} \backslash\{(\mathfrak{o}, \mathfrak{o})\}$ and then choosing $\vartheta$ as in (5.27a) with $p=2$ we obtain a probability plan $\operatorname{dil}_{\vartheta, 2}\left(\boldsymbol{\alpha}\llcorner\mathfrak{C} \backslash\{(\mathfrak{o}, \mathfrak{o})\})\right.$ in $\mathcal{H}_{=}^{2}\left(\mu_{1}, \mu_{2}\right)$ concentrated in $\mathfrak{C}[R] \backslash\{(\mathfrak{o}, \mathfrak{o})\}$ with the same cost $\int \mathrm{d}_{\mathfrak{C}}^{2} \mathrm{~d} \boldsymbol{\alpha}$. In order to show that Problem 7.4 has a solution we can then use the formulation (7.26) and prove that the set $C$ where the minimum will be found is narrowly compact in $\mathcal{P}(\mathfrak{C})$. Notice that the analogous property would not be true in $\mathcal{P}(Y \times Y)$ (unless $X$ is compact) since the collection of measures concentrated in $(X \times\{0\}) \times(X \times\{0\})$ would not be equally tight. Also the constraints $\mathfrak{h}_{i}^{2} \boldsymbol{\alpha}=\mu_{i}$ would not be preserved by narrow convergence, if one allows for arbitrary plans in $\mathcal{P}(\mathfrak{C})$, as in (7.23).

Theorem 7.6 (Existence of optimal plans for the HK problem) For every $\mu_{1}, \mu_{2} \in \mathcal{M}(X)$ the Hellinger-Kantorovich problem 7.4 always admits a solution $\boldsymbol{\alpha} \in \mathcal{P}(\mathfrak{C})$ concentrated on $\mathfrak{C}[R] \backslash\{(\mathfrak{o}, \mathfrak{o})\}$ with $R^{2}=\sum_{i} \mu_{i}(X)$.

Proof By the rescaling (7.27) it is not restrictive to look for minimizers $\boldsymbol{\alpha}$ of (7.26). Since $\mathfrak{C}[R]$ is closed in $\mathfrak{C}$ and the maps $r_{i}^{2}$ are continuous and bounded in $\mathfrak{C}[R], C$ is clearly narrowly closed. By Lemma 7.3, $C$ is also equally tight in $\mathcal{P}(\mathfrak{C})$, thus narrowly compact by Theorem 2.2 . Since the $d_{\mathfrak{C}}^{2}$ is 
lower semicontinuous in $\mathfrak{C}$, the existence of a minimizer of (7.26) then follows by the direct method of the calculus of variations.

We can also prove an interesting characterization of $\mathrm{HK}$ in terms of the Kantorovich-Wasserstein distance on $\mathcal{P}_{2}(\mathfrak{C})$ given by (7.14). An even deeper connection will be discussed in the next section, see Corollary 7.13.

Corollary 7.7 ( $\mathrm{HK}$ and the Kantorovich-Wasserstein distance on $\left.\mathcal{P}_{2}(\mathfrak{C})\right)$ For every $\mu_{1}, \mu_{2} \in \mathcal{M}(X)$ we have (recall the notation $\mathfrak{h}$ explained after (7.16))

$$
\mathrm{H}\left(\mu_{1}, \mu_{2}\right)=\min \left\{\mathrm{W}_{\mathfrak{d}_{\mathfrak{C}}}\left(\alpha_{1}, \alpha_{2}\right): \alpha_{i} \in \mathcal{P}_{2}(\mathfrak{C}), \quad \mathfrak{h}^{2} \alpha_{i}=\mu_{i}\right\},
$$

and there exist optimal measures $\bar{\alpha}_{i}$ for (7.28) concentrated on $\mathfrak{C}[R]$ with $R^{2}=\sum_{i} \mu_{i}(X)$. In particular the map $\mathfrak{h}^{2}: \mathcal{P}_{2}(\mathfrak{C}) \rightarrow \mathcal{M}(X)$ is a contraction, i.e.

$$
\mathrm{H}\left(\mathfrak{h}^{2} \alpha_{1}, \mathfrak{h}^{2} \alpha_{2}\right) \leq \mathrm{W}_{\mathrm{d}_{\mathfrak{C}}}\left(\alpha_{1}, \alpha_{2}\right) \text { for every } \alpha_{i} \in \mathcal{P}_{2}(\mathfrak{C}) .
$$

Proof If $\alpha_{i} \in \mathcal{P}_{2}(\mathfrak{C})$ with $\mathfrak{h}^{2} \alpha_{i}=\mu_{i}$ then any Kantorovich-Wasserstein optimal plan $\alpha \in \mathcal{P}(\mathfrak{C} \times \mathfrak{C})$ for (7.14) with marginals $\alpha_{i}$ clearly belongs to $\mathfrak{H}_{=}^{2}\left(\mu_{1}, \mu_{2}\right)$ and yields the bound $\mathrm{H}\left(\mu_{1}, \mu_{2}\right) \leq \mathrm{W}_{\mathrm{d}_{\mathfrak{C}}}\left(\alpha_{1}, \alpha_{2}\right)$. On the other hand, if $\boldsymbol{\alpha} \in \mathrm{Opt}_{\mathbb{H}_{\mathrm{K}}}\left(\mu_{1}, \mu_{2}\right)$ is an optimal solution for (7.23) and $\alpha_{i}:=\pi_{\sharp}^{i} \boldsymbol{\alpha} \in$ $\mathcal{P}_{2}(\mathfrak{C})$ are its marginals, we have $\mathbb{K}\left(\mu_{1}, \mu_{2}\right) \geq \mathbf{W}_{\mathrm{d}_{\mathfrak{C}}}\left(\alpha_{1}, \alpha_{2}\right)$, so that $\alpha_{i}$ realize the minimum for (7.28).

We conclude this section with two simple properties of the $\mathrm{H}$ functional. We denote by $\eta_{0}$ the null measure.

Lemma 7.8 (Subadditivity of $\mathrm{H}^{2}$ ) The functional $\mathrm{H}^{2}$ satisfies

$$
\mathrm{H}^{2}\left(\mu, \eta_{0}\right)=\mu(X), \mathrm{H}^{2}\left(\mu_{1}, \mu_{2}\right) \leq \mu_{1}(X)+\mu_{2}(X)
$$

for every $\mu, \mu_{i} \in \mathcal{M}(X)$, and it is subadditive, i.e. for every $\mu_{i}, \mu_{i}^{\prime} \in \mathcal{M}(X)$ we have

$$
\mathrm{H}^{2}\left(\mu_{1}+\mu_{1}^{\prime}, \mu_{2}+\mu_{2}^{\prime}\right) \leq \mathrm{H}^{2}\left(\mu_{1}, \mu_{2}\right)+\mathrm{H}^{2}\left(\mu_{1}^{\prime}, \mu_{2}^{\prime}\right) .
$$

Proof The relations in (7.30) are obvious. If $\boldsymbol{\alpha} \in \mathfrak{H}_{=}^{2}\left(\mu_{1}, \mu_{2}\right)$ and $\boldsymbol{\alpha}^{\prime} \in$ $\mathfrak{H}_{=}^{2}\left(\mu_{1}^{\prime}, \mu_{2}^{\prime}\right)$ it is easy to check that $\boldsymbol{\alpha}+\boldsymbol{\alpha}^{\prime} \in \mathfrak{H}_{=}^{2}\left(\mu_{1}+\mu_{1}^{\prime}, \mu_{2}+\mu_{2}^{\prime}\right)$. Since the cost functional is linear with respect to the plan, we get (7.31).

Subsequently we will use the symbol "L" for the restriction of measures.

Lemma 7.9 (A formulation with relaxed constraints) For every $\mu_{1}, \mu_{2} \in$ $\mathcal{M}(X)$ we have 


$$
\begin{aligned}
& \mathrm{HK}^{2}\left(\mu_{1}, \mu_{2}\right) \\
& =\min _{\boldsymbol{\alpha} \in \mathfrak{H}_{\leq}^{2}\left(\mu_{1}, \mu_{2}\right)}\left\{\int \mathrm{d}_{\mathfrak{C}^{2}}^{2}\left(\mathfrak{y}_{1}, \mathfrak{y}_{2}\right) \mathrm{d} \boldsymbol{\alpha}+\sum_{i}\left(\mu_{i}-\mathfrak{h}_{i}^{2} \boldsymbol{\alpha}\right)(X)\right\} \\
& =\mu_{1}(X)+\mu_{2}(X)-\max _{\boldsymbol{\alpha} \in \mathfrak{H}_{\leq}^{2}\left(\mu_{1}, \mu_{2}\right)}\left\{2 \int \mathrm{r}_{1} r_{2} \cos \left(\mathbf{d}_{\pi}\left(\mathbf{x}_{1}, \mathbf{x}_{2}\right)\right) \mathrm{d} \boldsymbol{\alpha}\right\} .
\end{aligned}
$$

\section{Moreover,}

(i) Equations (7.32a)-(7.32b) share the same class of optimal plans.

(ii) A plan $\alpha \in \mathfrak{H}_{\leq}^{2}\left(\mu_{1}, \mu_{2}\right)$ is optimal for (7.32a)-(7.32b) if and only if the plan $\boldsymbol{\alpha}_{\mathfrak{o}}:=\alpha\left\llcorner\left(\mathfrak{C}_{\mathfrak{o}} \times \mathfrak{C}_{\mathfrak{o}}\right)\right.$ is optimal as well.

(iii) If $\boldsymbol{\alpha}$ is optimal for (7.32a)-(7.32b) with $\mu_{i}^{\prime}:=\mu_{i}-\mathfrak{h}_{i}^{2} \boldsymbol{\alpha}$, then $\tilde{\boldsymbol{\alpha}}:=\boldsymbol{\alpha}+\boldsymbol{\alpha}^{\prime}$ is an optimal plan in $\operatorname{Opt}_{\mathbb{K}^{K}}\left(\mu_{1}, \mu_{2}\right)$ for all $\boldsymbol{\alpha}^{\prime} \in \mathfrak{H}_{=}^{2}\left(\mu_{1}^{\prime}, \mu_{2}^{\prime}\right)$.

(iv) A plan $\alpha \in \mathfrak{H}_{=}^{2}\left(\mu_{1}, \mu_{2}\right)$ belongs to $\operatorname{Opt}_{\mathbb{H}^{K}}\left(\mu_{1}, \mu_{2}\right)$ if and only if $\boldsymbol{\alpha}_{\mathfrak{o}}:=$ $\alpha\left\llcorner\left(\mathfrak{C}_{\mathfrak{o}} \times \mathfrak{C}_{\mathfrak{o}}\right)\right.$ is optimal for $(7.32 \mathrm{a})-(7.32 \mathrm{~b})$.

Proof The formulas (7.32a) and (7.32b) are just two different ways to write the same functional, since for every $\alpha \in \mathfrak{H}_{\leq}^{2}\left(\mu_{1}, \mu_{2}\right)$ we have

$$
\int \mathrm{d}_{\mathfrak{C}}^{2} \mathrm{~d} \boldsymbol{\alpha}+\sum_{i}\left(\mu_{i}-\mathfrak{h}_{i}^{2} \boldsymbol{\alpha}\right)(X)=\sum_{i} \mu_{i}(X)-2 \int \mathrm{r}_{1} \mathrm{r}_{2} \cos \left(\mathrm{d}_{\pi}\left(\mathrm{x}_{1}, \mathrm{x}_{2}\right)\right) \mathrm{d} \boldsymbol{\alpha}
$$

Thus, to prove (i) it is sufficient to show (7.32a). The inequality $\geq$ is obvious, since $\mathfrak{H}_{\leq}^{2}\left(\mu_{1}, \mu_{2}\right) \supset \mathfrak{H}_{=}^{2}\left(\mu_{1}, \mu_{2}\right)$ and for every $\boldsymbol{\alpha} \in \mathfrak{H}_{=}^{2}\left(\mu_{1}, \mu_{2}\right)$ the term $\sum_{i}\left(\mu_{i}-\mathfrak{h}_{i}^{2} \boldsymbol{\alpha}\right)(X)$ vanishes.

On the other hand, whenever $\alpha \in \mathfrak{H}_{\leq}^{2}\left(\mu_{1}, \mu_{2}\right)$, setting $\mu_{i}^{\prime \prime}:=\mathfrak{h}_{i}^{2} \boldsymbol{\alpha} \in \mathcal{M}(X)$, $\mu_{i}^{\prime}:=\mu_{i}-\mu_{i}^{\prime \prime}$ and observing that $\alpha \in \mathfrak{H}_{=}^{2}\left(\mu_{1}^{\prime \prime}, \mu_{2}^{\prime \prime}\right)$ we get

$$
\begin{aligned}
& \int \mathrm{d}_{\mathfrak{C}^{2}}^{2}\left(\mathfrak{y}_{1}, \mathfrak{y}_{2}\right) \mathrm{d} \boldsymbol{\alpha}+\sum_{i}\left(\mu_{i}-\mathfrak{h}_{i}^{2} \boldsymbol{\alpha}\right)(X) \geq \mathrm{H}^{2}\left(\mu_{1}^{\prime \prime}, \mu_{2}^{\prime \prime}\right)+\mu_{1}^{\prime}(X)+\mu_{2}^{\prime}(X) \\
& \quad \stackrel{(7.30)}{\geq} \mathrm{K}^{2}\left(\mu_{1}^{\prime}, \mu_{2}^{\prime}\right)+\mathrm{H}^{2}\left(\mu_{1}^{\prime \prime}, \mu_{2}^{\prime \prime}\right) \stackrel{(7.31)}{\geq} \mathrm{H}^{2}\left(\mu_{1}, \mu_{2}\right) .
\end{aligned}
$$

The same calculations also prove point (iii).

In order to check (ii) it is sufficient to observe that the integrand in (7.32b) vanishes on $\mathfrak{C} \backslash\left(\mathfrak{C}_{\mathfrak{o}} \times \mathfrak{C}_{\mathfrak{o}}\right)$.

Finally, if $\boldsymbol{\alpha} \in \mathrm{Opt}_{\mathbb{H}^{K}}\left(\mu_{1}, \mu_{2}\right)$ is optimal for (7.23), then by the consideration above it is optimal for (7.32b) and therefore (ii) shows that $\boldsymbol{\alpha}_{\mathfrak{o}}$ is optimal as well. The converse implication follows by (iii). 


\subsection{Gluing lemma and triangle inequality}

In this section we will prove that $\mathrm{HK}$ satisfies the triangle inequality and therefore is a distance on $\mathcal{M}(X)$. As in Optimal Transport (see e.g. [2, Sect. 7.1]), the triangle inequality can be obtained by a gluing technique that allows us to join a couple of optimal transport plans with a common marginal. Here we will deal with transport plans on the cone $\mathfrak{C}$ and homogeneous marginals. We will also consider a more general situation where a sequence of measures is involved: it will turn out to be extremely useful in various topological (Theorem 7.15) and metric (Theorems 7.17, 8.4, 8.6, 8.8) results.

The main technical step is provided by the following property for plans in $\mathcal{M}\left(\mathfrak{C}^{\otimes N}\right)$ with given homogeneous marginals, which is a simple application of the rescaling invariance in (7.27). This property is nontrivial since homogeneous marginals are considerably less rigid than the usual marginals and therefore the gluing technique requires a preliminary normalization, which does not affect the computation of the $\mathrm{H}$ distance.

Lemma 7.10 (Normalization of lifts) Let $\boldsymbol{\alpha} \in \mathcal{M}_{2}\left(\mathfrak{C}^{\otimes N}\right), N \geq 2$, be a plan satisfying

$$
\begin{aligned}
& \mathfrak{h}_{i}^{2} \boldsymbol{\alpha}=\mu_{i} \in \mathcal{M}(X) \text { for } i=1, \ldots, N, \\
& \text { and } a_{i}=\int \mathrm{d}_{\mathfrak{C}}^{2}\left(\mathfrak{y}_{i-1}, \mathfrak{y}_{i}\right) \mathrm{d} \boldsymbol{\alpha} \text { for } i=2, \ldots, N,
\end{aligned}
$$

and let $j \in\{1, \ldots, N\}$ be fixed. Then, it is possible to find a new plan $\bar{\alpha} \in$ $\mathcal{M}_{2}\left(\mathfrak{C}^{\otimes N}\right)$ which still satisfies (7.34) and additionally the normalization of the jth lift,

$$
\pi_{\sharp}^{j}(\overline{\boldsymbol{\alpha}})=\delta_{\mathfrak{o}}+\mathfrak{p}_{\sharp}\left(\mu_{j} \otimes \delta_{1}\right) .
$$

Proof By possibly adding $\otimes^{N} \delta_{\mathfrak{o}}$ to $\boldsymbol{\alpha}$ (which does not modify (7.34)) we may suppose that

$$
\omega_{j}:=\alpha\left(\left\{\mathfrak{y} \in \mathfrak{C}^{\otimes N}: \pi^{j}(\mathfrak{y})=\mathfrak{o}\right\}\right) \geq 1,
$$

where $j$ is fixed as in the lemma. In order to find $\bar{\alpha}$ it is sufficient to rescale $\alpha$ by the function

$$
\vartheta(\mathfrak{y}):= \begin{cases}\mathrm{r}_{j}(\mathfrak{y}) & \text { if } \mathfrak{y}_{j} \neq \mathfrak{o}, \\ \omega_{j}^{-1 / 2} & \text { otherwise. }\end{cases}
$$

With the notation of (7.17) we set $\overline{\boldsymbol{\alpha}}:=\operatorname{dil}_{\vartheta, 2}(\boldsymbol{\alpha})$ and we decompose $\boldsymbol{\alpha}$ in the sum $\boldsymbol{\alpha}=\boldsymbol{\alpha}^{\prime}+\boldsymbol{\alpha}^{\prime \prime}$ where $\boldsymbol{\alpha}^{\prime}=\boldsymbol{\alpha}\left\llcorner\left\{\mathfrak{y} \in \mathfrak{C}^{\otimes N}: \pi^{j}(\mathfrak{y})=\mathfrak{o}\right\}\right.$. For every $\zeta \in \mathrm{B}_{b}(\mathfrak{C})$ we have 


$$
\begin{aligned}
\int \zeta(\mathfrak{y} j) \mathrm{d} \overline{\boldsymbol{\alpha}} & =\int \zeta\left(\mathfrak{y} j \cdot \vartheta^{-1}(\mathfrak{y})\right) \vartheta^{2}(\mathfrak{y}) \mathrm{d} \boldsymbol{\alpha} \\
& =\int \zeta(\mathfrak{o}) \omega_{j}^{-1} \mathrm{~d} \boldsymbol{\alpha}^{\prime}+\int \zeta\left(\left[x_{j}, r_{j} / \vartheta(\mathfrak{y})\right]\right) \vartheta^{2}(\mathfrak{y}) \mathrm{d} \boldsymbol{\alpha}^{\prime \prime} \\
& =\zeta(\mathfrak{o})+\int \zeta\left(\left[x_{j}, 1\right]\right) \mathrm{r}_{j}^{2} \mathrm{~d} \boldsymbol{\alpha}^{\prime \prime}=\zeta(\mathfrak{o})+\int \zeta\left(\left[x_{j}, 1\right]\right) \mathrm{r}_{j}^{2} \mathrm{~d} \boldsymbol{\alpha} \\
& =\zeta(\mathfrak{o})+\int \zeta \circ \mathfrak{p} \mathrm{d}\left(\mu_{j} \otimes \delta_{1}\right)
\end{aligned}
$$

which yields (7.35).

We can now prove a general form of the so-called "gluing lemma" that is the natural extension of the well known result for transport problems (see e.g. [2, Lem. 5.3.4]). Here its formulation is strongly related to the rescaling invariance of optimal plans given by Lemma 7.10.

Lemma 7.11 (Gluing lemma) Let us consider a finite collection of measures $\mu_{i} \in \mathcal{M}(X)$ for $i=1, \ldots, N$ with $N \geq 2$. Set

$$
\Theta:=\sqrt{\mu_{1}(X)}+\sum_{i=2}^{N} \mathrm{H}\left(\mu_{i-1}, \mu_{i}\right) \text { and } M^{2}:=\sum_{i=1}^{N} \mu_{i}(X) .
$$

Then there exist plans $\boldsymbol{\alpha}_{1}, \boldsymbol{\alpha}_{2} \in \mathcal{P}_{2}\left(\mathfrak{C}^{\otimes N}\right)$ such that

$$
\begin{aligned}
& \mathfrak{h}_{i}^{2} \boldsymbol{\alpha}_{k}=\mu_{i} \text { for } i=1, \ldots, N \text { and } \\
& \quad \int \mathrm{d}_{\mathfrak{C}}^{2}\left(\mathfrak{y}_{i-1}, \mathfrak{y}_{i}\right) \mathrm{d} \boldsymbol{\alpha}_{k}=\mathrm{K}^{2}\left(\mu_{i-1}, \mu_{i}\right) \text { for } i=2, \ldots, N .
\end{aligned}
$$

Moreover, the plans $\boldsymbol{\alpha}_{k}$ satisfy the following additional conditions:

$$
\begin{aligned}
& \boldsymbol{\alpha}_{1} \text { is concentrated on }\left\{\mathfrak{y} \in \mathfrak{C}^{\otimes N}: \sum_{i} \mathrm{r}_{i}^{2}(\mathfrak{y}) \leq M^{2}\right\} \\
& \boldsymbol{\alpha}_{2} \text { is concentrated on }\left\{\mathfrak{y} \in \mathfrak{C}^{\otimes N}: \sup _{i} \mathrm{r}_{i}(\mathfrak{y}) \leq \Theta\right\}=(\mathfrak{C}[\Theta])^{\otimes N} .
\end{aligned}
$$

Proof We first construct a plan $\boldsymbol{\alpha}$ satisfying (7.38), then suitable rescalings will provide $\boldsymbol{\alpha}_{k}$ satisfying (7.39) or (7.40). In order to clarify the argument, we consider $N$-copies $X_{1}, X_{2}, \ldots, X_{N}$ of $X$ (and for $\mathfrak{C}$ in a similar way) so that $X^{\otimes N}=\prod_{i=1}^{N} X_{i}$.

We argue by induction; the starting case $N=2$ is covered by Theorem 7.6. Let us now discuss the induction step, by assuming that the thesis holds for $N$ and proving it for $N+1$. We can thus find an optimal plan $\boldsymbol{\alpha}^{N}$ such that (7.38) 
hold, and another optimal plan $\boldsymbol{\alpha} \in \mathrm{Opt}_{\mathbb{K}_{\mathrm{K}}}\left(\mu_{N}, \mu_{N+1}\right)$ for the pair $\mu_{N}, \mu_{N+1}$. Applying the normalization Lemma 7.10 to $\boldsymbol{\alpha}^{N}$ (with $j=N$ ) and to $\boldsymbol{\alpha}$ (with $j=1$ ) we can assume that

$$
\pi_{\sharp}^{N}\left(\boldsymbol{\alpha}^{N}\right)=\delta_{\mathfrak{o}}+\mathfrak{p}_{\sharp}\left(\mu_{N} \otimes \delta_{1}\right)=\pi_{\sharp}^{1}(\boldsymbol{\alpha}) .
$$

Therefore we can apply the standard gluing Lemma in $\left(\prod_{i=1}^{N-1} \mathfrak{C}_{i}\right), \mathfrak{C}_{N}, \mathfrak{C}_{N+1}$ (see e.g. [2, Lemma 5.3.2] and [1, Lemma 2.2] in the case of arbitrary topological spaces) obtaining a new plan $\boldsymbol{\alpha}^{N+1}$ satisfying $\pi_{\sharp}^{1,2, \cdots, N} \boldsymbol{\alpha}^{N+1}=\boldsymbol{\alpha}^{N}$ and $\pi^{N, N+1} \boldsymbol{\alpha}^{N+1}=\boldsymbol{\alpha}$. In particular, $\boldsymbol{\alpha}^{N+1}$ satisfies (7.38).

A further application of the rescaling (7.27) with $\vartheta$ as in (5.27a) yields a plan $\boldsymbol{\alpha}_{1}$ satisfying also (7.39).

In order to obtain $\boldsymbol{\alpha}_{2}$, we can assume $\boldsymbol{\alpha}(\{|\mathfrak{y}|=0\})=0$ and set $\boldsymbol{\alpha}_{2}=$ $\operatorname{dil}_{\vartheta, 2}(\boldsymbol{\alpha})$, where we use the rescaling function

$$
\vartheta(\mathfrak{y}):=r^{-1}|\mathfrak{y}|_{\infty}=r^{-1} \sup _{i} \boldsymbol{r}_{i}(\mathfrak{y}) \text { with } r^{2}:=\int_{\mathfrak{C}^{\otimes N}}|\mathfrak{y}|_{\infty}^{2} \mathrm{~d} \boldsymbol{\alpha} .
$$

To obtain (7.40) it remains to estimate $r$. We consider arbitrary coefficients $\theta_{i}>0$ and use for $n=2, \ldots, N$ the inequality

$$
\begin{aligned}
\mathrm{r}_{n} & \leq \mathrm{r}_{1}+\sum_{i=2}^{n}\left|\mathrm{r}_{i}-\mathrm{r}_{i-1}\right| \leq\left(\sum_{i=1}^{n} \theta_{i}^{-1}\right)^{1 / 2}\left(\theta_{1} \mathrm{r}_{1}^{2}+\sum_{i=2}^{n} \theta_{i}\left|\mathrm{r}_{i}-\mathrm{r}_{i-1}\right|^{2}\right)^{1 / 2} \\
& \leq\left(\sum_{i=1}^{N} \theta_{i}^{-1}\right)^{1 / 2}\left(\theta_{1} \mathrm{r}_{1}^{2}+\sum_{i=2}^{N} \theta_{i} \mathrm{~d}_{\mathfrak{C}}^{2}\left(\mathfrak{y}_{i}, \mathfrak{y}_{i-1}\right)\right)^{1 / 2}
\end{aligned}
$$

which yields

$$
\sup _{i} \boldsymbol{r}_{i}(\mathfrak{y}) \leq\left(\sum_{i=1}^{N} \theta_{i}^{-1}\right)^{1 / 2}\left(\theta_{1} \mathrm{r}_{1}^{2}+\sum_{i=2}^{N} \theta_{i} \mathrm{~d}_{\mathfrak{C}}^{2}\left(\mathfrak{y}_{i}, \mathfrak{y}_{i-1}\right)\right)^{1 / 2},
$$

and therefore

$$
\begin{aligned}
r^{2} & =\int_{\mathfrak{C}^{\otimes N}}|\mathfrak{y}|_{\infty}^{2} \mathrm{~d} \boldsymbol{\alpha} \leq\left(\sum_{i=1}^{N} \theta_{i}^{-1}\right) \int_{\mathfrak{C}^{\otimes N}}\left(\theta_{1} \mathrm{r}_{1}^{2}+\sum_{i=2}^{N} \theta_{i} \mathrm{~d}_{\mathfrak{C}^{2}}^{2}\left(\mathfrak{y}_{i}, \mathfrak{y}_{i-1}\right)\right) \mathrm{d} \boldsymbol{\alpha} \\
& =\left(\sum_{i=1}^{N} \theta_{i}^{-1}\right) \cdot\left(\theta_{1} \mu_{1}(X)+\sum_{i=2}^{N} \theta_{i} \mathrm{H}^{2}\left(\mu_{i-1}, \mu_{i}\right)\right) ;
\end{aligned}
$$

optimizing with respect to $\theta_{i}>0$ we obtain the value of $\Theta$ given by (7.37). 
The next remark gives a similar rescaling result for probability couplings $\boldsymbol{\beta} \in \mathcal{P}_{2}\left(\mathfrak{C}^{\otimes N}\right)$.

Remark 7.12 In a completely similar way (see [2, Lemma 5.3.4]), for every $N \geq 2$ and every finite collection of measures $\mu_{i} \in \mathcal{M}(X)$, there exists a plan $\boldsymbol{\beta} \in \mathcal{P}_{2}\left(\mathfrak{C}^{\otimes N}\right)$ concentrated on $\left\{\mathfrak{y} \in \mathfrak{C}^{\otimes N}: \sup _{i} \mathrm{r}_{i}(\mathfrak{y}) \leq \Xi\right\}$ with

$$
\Xi:=\sqrt{\mu_{1}(X)}+\sum_{i=2}^{N} \mathrm{~K}\left(\mu_{1}, \mu_{i}\right),
$$

such that

$$
\mathfrak{h}_{i}^{2} \boldsymbol{\beta}=\mu_{i} \text { and } \int \mathrm{d}_{\mathfrak{C}}^{2}\left(\mathfrak{y}_{1}, \mathfrak{y}_{i}\right) \mathrm{d} \boldsymbol{\beta}=\mathrm{H}^{2}\left(\mu_{1}, \mu_{i}\right)
$$

for $i=1, \ldots, N$.

Arguing as in the proof of Corollary 7.7 one immediately obtains the following result, which will be needed for the proof of Theorem 8.8 and for the subsequent corollary.

Corollary 7.13 For every finite collection of measures $\mu_{i} \in \mathcal{M}(X), i=$ $1, \ldots, N$, there exist $\alpha_{i}, \beta_{i} \in \mathcal{P}_{2}(\mathfrak{C})$ with $\alpha_{i}$ concentrated in $\mathfrak{C}[r]$ where $r=\min (M, \Theta)$ is given as in (7.37) and $\beta_{i}$ concentrated in $\mathfrak{C}[\Xi]$ given by (7.41) such that

$$
\begin{aligned}
\mathfrak{h}^{2} \alpha_{i}=\mu_{i} \text { and } & \mathfrak{h}^{2} \beta_{i}=\mu_{i} \text { for } i=1, \ldots, N, \\
\mathrm{H}\left(\mu_{1}, \mu_{i}\right)= & \mathbf{W}_{\mathrm{d}_{\mathfrak{C}}}\left(\beta_{1}, \beta_{i}\right) \text { and } \\
\mathrm{HK}\left(\mu_{i}, \mu_{i+1}\right)= & \mathbf{W}_{\mathrm{d}_{\mathfrak{C}}}\left(\alpha_{i}, \alpha_{i+1}\right) \text { for } i=2, \ldots, N .
\end{aligned}
$$

We are now in the position to show that the functional $\mathrm{HK}$ is a true distance on $\mathcal{M}(X)$, where we deduce the triangle inequality from that for $\mathrm{W}_{\mathrm{d}_{\mathfrak{C}}}$ by using normalized lifts.

Corollary 7.14 ( $\mathrm{HK}$ is a distance) $\mathrm{H}$ is a distance on $\mathcal{M}(X)$; in particular, for every $\mu_{1}, \mu_{2}, \mu_{3} \in \mathcal{M}(X)$ we have the triangle inequality

$$
\mathrm{H}\left(\mu_{1}, \mu_{3}\right) \leq \mathrm{H}\left(\mu_{1}, \mu_{2}\right)+\mathrm{H}\left(\mu_{2}, \mu_{3}\right) .
$$

Proof It is immediate to check that $\mathrm{H}$ is symmetric and $\mathrm{H}\left(\mu_{1}, \mu_{2}\right)=0$ if and only if $\mu_{1}=\mu_{2}$. In order to check (7.43) it is sufficient to apply the previous corollary 7.13 to find measures $\alpha_{i} \in \mathcal{P}_{2}(\mathfrak{C}), i=1,2,3$, such that 
$\mathfrak{h}^{2} \alpha_{i}=\mu_{i}$ and $\mathbf{H}\left(\mu_{1}, \mu_{2}\right)=\mathbf{W}_{\mathrm{d}_{\mathfrak{C}}}\left(\alpha_{1}, \alpha_{2}\right)$ and $\mathbf{H}\left(\mu_{2}, \mu_{3}\right)=\mathbf{W}_{\mathrm{d}_{\mathfrak{C}}}\left(\alpha_{2}, \alpha_{3}\right)$. Applying the triangle inequality for $\mathrm{W}_{\mathfrak{d}_{\mathfrak{C}}}$ we obtain

$$
\begin{aligned}
\mathrm{HK}\left(\mu_{1}, \mu_{3}\right) & \leq \mathrm{W}_{\mathrm{d}_{\mathfrak{C}}}\left(\alpha_{1}, \alpha_{3}\right) \leq \mathrm{W}_{\mathrm{d}_{\mathfrak{C}}}\left(\alpha_{1}, \alpha_{2}\right)+\mathrm{W}_{\mathrm{d}_{\mathfrak{C}}}\left(\alpha_{2}, \alpha_{3}\right) \\
& =\mathrm{HK}\left(\mu_{1}, \mu_{2}\right)+\mathrm{H}\left(\mu_{2}, \mu_{3}\right) .
\end{aligned}
$$

As a consequence of the previous two results, the map $\mathfrak{h}^{2}: \mathcal{P}_{2}(\mathfrak{C}) \rightarrow \mathcal{M}(X)$ is a metric submersion.

\subsection{Metric and topological properties}

In this section we will assume that the topology $\tau$ on $X$ is induced by $\mathrm{d}$ and that $(X, \mathrm{~d})$ is separable, so that also $\left(\mathfrak{C}, \mathrm{d}_{\mathfrak{C}}\right)$ is separable. Notice that in this case there is no difference between weak and narrow topology in $\mathcal{M}(X)$. Moreover, since $X$ is separable, $\mathcal{M}(X)$ equipped with the weak topology is metrizable, so that converging sequences are sufficient to characterize the weak-narrow topology.

It turns out [2, Chap. 7] that $\left(\mathcal{P}_{2}(\mathfrak{C}), W_{\mathfrak{d}_{\mathfrak{C}}}\right)$ is a separable metric space: convergence of a sequence $\left(\alpha_{n}\right)_{n \in \mathbb{N}}$ to a limit measure $\alpha$ in $\left(\mathcal{P}_{2}(\mathfrak{C}), \mathrm{W}_{\mathrm{d}_{\mathfrak{C}}}\right)$ corresponds to weak-narrow convergence in $\mathcal{P}(\mathfrak{C})$ and convergence of the quadratic moments, or, equivalently, to convergence of integrals of continuous functions with quadratic growth, i.e.

$$
\lim _{n \rightarrow \infty} \int_{\mathfrak{C}} \varphi \mathrm{d} \alpha_{n}=\int_{\mathfrak{C}} \varphi \mathrm{d} \alpha \quad \text { for every } \varphi \in \mathrm{C}(\mathfrak{C}) \text { with }|\varphi(\mathfrak{y})| \leq A+B \mathrm{r}^{2}(\mathfrak{y}),
$$

for some constants $A, B \geq 0$ depending on $\varphi$. Recall that $\mathrm{r}^{2}(\mathfrak{y})=\mathrm{d}_{\mathfrak{C}}^{2}(\mathfrak{y}, \mathfrak{o})$.

Theorem 7.15 ( $\mathrm{H}$ metrizes the weak topology on $\mathcal{M}(X)) \mathrm{HK}$ induces the weak-narrow topology on $\mathcal{M}(X)$ : a sequence $\left(\mu_{n}\right)_{n \in \mathbb{N}} \in \mathcal{M}(X)$ converges to a measure $\mu$ in $(\mathcal{M}, \mathrm{K})$ if and only if $\left(\mu_{n}\right)_{n \in \mathbb{N}}$ converges weakly to $\mu$ in duality with continuous and bounded functions.

In particular, the metric space $(\mathcal{M}(X), \mathrm{H})$ is separable.

Proof Let us first suppose that $\lim _{n \rightarrow \infty} \mathrm{HK}\left(\mu_{n}, \mu\right)=0$. We argue by contradiction and we assume that there exists a function $\zeta \in \mathrm{C}_{b}(X)$ and a subsequence (still denoted by $\mu_{n}$ ) such that

$$
\inf _{n}\left|\int_{X} \zeta \mathrm{d} \mu_{n}-\int_{X} \zeta \mathrm{d} \mu\right|>0
$$


The first estimate of (7.30) and the triangle inequality show that

$$
\limsup _{n \rightarrow \infty} \mu_{n}(X) \leq \limsup _{n \rightarrow \infty}\left(\mathrm{K}\left(\mu_{n}, \mu\right)+\mathrm{K}\left(\mu, \eta_{0}\right)\right)^{2}=\mu(X),
$$

so that $\sup _{n} \mu_{n}(X)=M^{2}<\infty$. By Corollary 7.7 we can find measures $\alpha_{n}, \alpha_{n}^{\prime} \in \mathcal{P}_{2}(\mathfrak{C})$ concentrated on $\mathfrak{C}[2 M]$ such that

$$
\mathfrak{h}^{2} \alpha_{n}=\mu, \quad \mathfrak{h}^{2} \alpha_{n}^{\prime}=\mu_{n}, \quad \mathbf{W}_{\mathrm{d}_{\mathfrak{C}}}\left(\alpha_{n}, \alpha_{n}^{\prime}\right)=\mathrm{H}\left(\mu, \mu_{n}\right) .
$$

By Lemma 7.3 the sequence $\left(\alpha_{n}\right)_{n \in \mathbb{N}}$ is equally tight in $\mathcal{P}_{2}(\mathfrak{C})$; since it is also uniformly bounded there exists a subsequence $k \mapsto n_{k}$ such that $\alpha_{n_{k}}$ weakly converges to a limit $\alpha \in \mathcal{P}_{2}(\mathfrak{C})$. Since $\alpha_{n}$ is concentrated on $\mathfrak{C}[2 M]$ we also have $\lim _{k \rightarrow \infty} \mathrm{W}_{\mathfrak{d}_{\mathfrak{C}}}\left(\alpha_{n_{k}}, \alpha\right)=0$ and therefore $\mathfrak{h}^{2} \alpha=\mu$, $\lim _{k \rightarrow \infty} \mathrm{W}_{\mathrm{d}_{\mathfrak{C}}}\left(\alpha_{n_{k}}^{\prime}, \alpha\right)=0$.

We thus have

$$
\lim _{k \rightarrow \infty} \int_{X} \zeta(x) \mathrm{d} \mu_{n_{k}}=\lim _{k \rightarrow \infty} \int_{\mathfrak{C}} \zeta(\mathbf{x}) \mathrm{r}^{2} \mathrm{~d} \alpha_{n_{k}}^{\prime}=\int_{\mathfrak{C}} \zeta(\mathbf{x}) \mathrm{r}^{2} \mathrm{~d} \alpha=\int_{X} \zeta(x) \mathrm{d} \mu
$$

which contradicts (7.45).

In order to prove the converse implication, let us suppose that $\mu_{n}$ is converging weakly to $\mu$ in $\mathcal{N}(X)$. If $\mu$ is the null measure $\eta_{0}$, then $\lim _{n \rightarrow \infty} \mu_{n}(X)=0$ so that $\lim _{n \rightarrow \infty} \mathrm{HK}\left(\mu_{n}, \mu\right)=0$ by (7.30).

So we can suppose that $m:=\mu(X)>0$ and have $m_{n}:=\mu_{n}(X) \geq m / 2>0$ for sufficiently large $n$. We now consider the measures $\alpha_{n}, \alpha \in \mathcal{P}(\mathfrak{C})$ given by

$$
\alpha_{n}:=\mathfrak{p}_{\sharp}\left(m_{n}^{-1} \mu_{n} \otimes \delta_{\sqrt{m_{n}}}\right) \text { and } \alpha:=\mathfrak{p}_{\sharp}\left(m^{-1} \mu \otimes \delta_{\sqrt{m}}\right) .
$$

Since $\mathfrak{h}^{2} \alpha_{n}=\mu_{n}$ and $\mathfrak{h}^{2} \alpha=\mu$, by (7.29) we have $\mathrm{H}\left(\mu_{n}, \mu\right) \leq \mathbf{W}_{\mathfrak{d}_{\mathfrak{C}}}\left(\alpha_{n}, \alpha\right)$.

Since $m_{n}^{-1} \mu_{n}$ is weakly converging to $m^{-1} \mu$ in $\mathcal{P}(X)$ and $m_{n} \rightarrow m$, it is easy to check that $m_{n}^{-1} \mu_{n} \otimes \delta_{\sqrt{m_{n}}}$ weakly converges to $m^{-1} \mu \otimes \delta_{\sqrt{m}}$ in $\mathcal{P}(Y)$ and therefore $\alpha_{n}$ weakly converges to $\alpha$ in $\mathcal{P}(\mathfrak{C})$ by the continuity of the projection $\mathfrak{p}$. Hence, in order to conclude that $\mathrm{W}_{\mathfrak{d}_{\mathfrak{C}}}\left(\alpha_{n}, \alpha\right) \rightarrow 0$ it is now sufficient to prove the convergence of their quadratic moments with respect to the vertex $\mathfrak{o}$. However, this is is immediate because of

$$
\lim _{n \rightarrow \infty} \int \mathrm{d}_{\mathfrak{C}^{2}}^{2}(\mathfrak{y}, \mathfrak{o}) \mathrm{d} \alpha_{n}=\lim _{n \rightarrow \infty} \int \mathrm{r}^{2} \mathrm{~d} \alpha_{n}=\lim _{n \rightarrow \infty} m_{n}=m=\int \mathrm{d}_{\mathfrak{C}^{\mathfrak{c}}}^{2}(\mathfrak{y}, \mathfrak{o}) \mathrm{d} \alpha .
$$

Corollary 7.16 (Compactness) If $(X, \mathrm{~d})$ is a compact metric space then $(\mathcal{M}(X), \mathrm{H})$ is a proper metric space, i.e. every bounded set is relatively compact. 
Proof It is sufficient to notice that a set $\mathcal{C} \subset \mathcal{M}(X)$ is bounded w.r.t. $\mathrm{H}$ if and only if $\sup _{\mu \in \mathcal{C}} \mu(X)<\infty$. Then the classical weak sequential compactness of closed bounded sets in $\mathcal{M}(X)$ gives the result.

The following completeness result for $(\mathcal{M}(X), \mathrm{H})$ is obtained by suitable liftings of measures $\mu_{i}$ to probability measures $\alpha_{i} \in \mathcal{P}_{2}(\mathfrak{C})$, supported in some $\mathfrak{C}[\Theta]$. Then the completeness of the Kantorovich-Wasserstein space $\left(\mathcal{P}_{2}(\mathfrak{C}), W_{d_{\mathfrak{C}}}\right)$ is exploited.

Theorem 7.17 (Completeness of $(\mathcal{N}(X), \mathrm{HK}))$ If $(X, \mathrm{~d})$ is complete than the metric space $(\mathcal{N}(X), \mathrm{HK})$ is complete.

Proof We have to prove that every Cauchy sequence $\left(\mu_{n}\right)_{n \in \mathbb{N}}$ in $(\mathcal{M}(X), \mathrm{H})$ admits a convergent subsequence. By exploiting the Cauchy property, we can find an increasing sequence of integers $k \mapsto n(k)$ such that $\mathrm{HK}\left(\mu_{m}, \mu_{m^{\prime}}\right) \leq$ $2^{-k}$ whenever $m, m^{\prime} \geq n(k)$ and we consider the subsequence $\mu_{i}^{\prime}:=\mu_{n(i)}$, so that

$$
\sqrt{\mu_{1}(X)}+\sum_{i=2}^{N} \mathrm{H}\left(\mu_{n(i)}, \mu_{n(i-1)}\right) \leq \sqrt{\mu_{1}(X)}+1 .
$$

By applying the Gluing Lemma 7.11, for every $N>0$ we can find measures $\alpha_{i}^{N} \in \mathcal{P}_{2}(\mathfrak{C}), i=1, \ldots, N$, concentrated on $\mathfrak{C}[\Theta]$ with $\Theta:=\sqrt{\mu_{1}(X)}+1$, such that $\mathfrak{h}^{2} \alpha_{i}^{N}=\mu_{i}^{\prime}$ and $\mathrm{W}_{\mathrm{d}_{\mathfrak{C}}}\left(\alpha_{i}^{N}, \alpha_{i-1}^{N}\right)=\mathrm{HK}\left(\mu_{i}^{\prime}, \mu_{i-1}^{\prime}\right)$.

For every $i$ the sequence $N \mapsto \alpha_{i}^{N} \in \mathcal{P}_{2}(\mathfrak{C})$ is equally tight by Lemma 7.3 and concentrated on the bounded set $\mathfrak{C}[\Theta]$, so that by Prokhorov Theorem it is relatively compact in $\left(\mathcal{P}_{2}(\mathfrak{C}), \mathrm{W}_{\mathrm{d}_{\mathfrak{C}}}\right)$.

By a standard diagonal argument, we can find a further increasing subsequence $m \mapsto N(m)$ and limit measures $\alpha_{i} \in \mathcal{P}_{2}(\mathfrak{C})$ such that $\lim _{m \rightarrow \infty} \mathbf{W}_{\mathrm{d}_{\mathfrak{C}}}\left(\alpha_{i}^{N(m)}, \alpha_{i}\right)=0$. The convergence with respect to $\mathbf{W}_{\mathrm{d}_{\mathfrak{C}}}$ yields that

$$
\mathfrak{h}^{2} \alpha_{i}=\mu_{i}, \quad \mathrm{~W}_{\mathrm{d}_{\mathfrak{C}}}\left(\alpha_{i}, \alpha_{i-1}\right)=\mathrm{HK}\left(\mu_{i}^{\prime}, \mu_{i-1}^{\prime}\right) \leq 2^{i-1} .
$$

It follows that $i \mapsto \alpha_{i}$ is a Cauchy sequence in $\left(\mathcal{P}_{2}(\mathfrak{C}), \mathrm{W}_{\mathrm{d}_{\mathfrak{C}}}\right)$ which is a complete metric space [2, Prop. 7.1.5] and therefore there exists $\alpha \in \mathcal{P}_{2}(\mathfrak{C})$ such that $\lim _{i \rightarrow \infty} \mathrm{W}_{\mathrm{d}_{\mathfrak{C}}}\left(\alpha_{i}, \alpha\right)=0$. Setting $\mu:=\mathfrak{h}^{2} \alpha \in \mathcal{M}(X)$ we thus obtain $\lim _{i \rightarrow \infty} \mathrm{H}\left(\mu_{i}^{\prime}, \mu\right)=0$.

We conclude this section by proving a simple comparison estimate for $\mathrm{HK}$ with the Bounded Lipschitz metric (cf. [19, Sect. 11.3]), see also [27, Thm. 3], and the flat metric. The Bounded Lipschitz metric is defined via 
$\operatorname{BL}\left(\mu_{1}, \mu_{2}\right):=\sup \left\{\int \xi \mathrm{d}\left(\mu_{1}-\mu_{2}\right): \xi \in \operatorname{Lip}_{b}(X), \sup _{X}|\xi|+\operatorname{Lip}(\xi, X) \leq 1\right\}$

and it is metrically equivalent to the flat metric

$\widetilde{\mathrm{BL}}\left(\mu_{1}, \mu_{2}\right):=\sup \left\{\int \xi \mathrm{d}\left(\mu_{1}-\mu_{2}\right): \xi \in \operatorname{Lip}_{b}(X), \sup _{X}|\xi| \vee \operatorname{Lip}(\xi, X) \leq 1\right\}$,

in the sense that $\mathrm{BL}\left(\mu_{1}, \mu_{2}\right) \leq \widetilde{\mathrm{BL}}\left(\mu_{1}, \mu_{2}\right) \leq 2 \mathrm{BL}\left(\mu_{1}, \mu_{2}\right)$. In its turn, $\widetilde{\mathrm{BL}}\left(\mu_{1}, \mu_{2}\right)$ coincides with the Piccoli-Rossi distance we considered in Example E.9 of Sect. 3.3, see [39]. We do not claim that the constant $C_{*}$ below is optimal.

Proposition 7.18 For every $\mu_{1}, \mu_{2} \in \mathcal{N}(X)$ we have

$$
\widetilde{\mathrm{BL}}\left(\mu_{1}, \mu_{2}\right) \leq C_{*}\left(\sum_{i} \mu_{i}(X)\right)^{1 / 2} \mathrm{~K}\left(\mu_{1}, \mu_{2}\right) \text {, where } C_{*}:=\sqrt{2+\pi^{2} / 2}
$$

Proof Let $\xi \in \operatorname{Lip}_{b}(X)$ with $\sup _{X}|\xi| \leq 1$ and $\operatorname{Lip}(\xi, X) \leq 1$, and let $\boldsymbol{\alpha} \in$ $\mathcal{P}(\mathfrak{C})$ optimal for (7.26) and concentrated on $\mathfrak{C}[R]$ with $\overline{R^{2}}:=\mu_{1}\left(X_{1}\right)+$ $\mu_{2}\left(X_{2}\right)$. Notice that

$$
\begin{aligned}
\left|\xi\left(x_{1}\right)-\xi\left(x_{2}\right)\right| & \leq \min \left(\mathrm{d}\left(x_{1}, x_{2}\right), 2\right) \leq 2 \mathrm{~d}_{2}\left(x_{1}, x_{2}\right) \leq 2 \mathrm{~d}_{\pi}\left(x_{1}, x_{2}\right) \\
& \leq 2 \pi \sin \left(\mathrm{d}_{\pi}\left(x_{1}, x_{2}\right) / 2\right) .
\end{aligned}
$$

We consider the function $\zeta: \mathfrak{C} \rightarrow \mathbb{R}$ defined by $\zeta(\mathfrak{y}):=\xi(\mathbf{x}) \mathrm{r}^{2}$. Hence, $\zeta$ satisfies

$$
\begin{aligned}
\left|\zeta\left(\mathfrak{y}_{1}\right)-\zeta\left(\mathfrak{y}_{2}\right)\right| & \leq\left|\xi\left(\mathrm{x}_{1}\right)-\xi\left(\mathrm{x}_{2}\right)\right| \mathrm{r}_{1} \mathrm{r}_{2}+\left(\left|\xi\left(\mathrm{x}_{1}\right)\right| \mathrm{r}_{1}+\left|\xi\left(\mathrm{x}_{2}\right)\right| \mathrm{r}_{2}\right)\left|\mathrm{r}_{1}-\mathrm{r}_{2}\right| \\
& \leq 2 \pi \sin \left(\mathrm{d}_{\pi}\left(\mathrm{x}_{1}, \mathrm{x}_{2}\right) / 2\right) \mathrm{r}_{1} \mathrm{r}_{2}+\left(\mathrm{r}_{1}+\mathrm{r}_{2}\right)\left|\mathrm{r}_{1}-\mathrm{r}_{2}\right| \\
& \stackrel{(7.4)}{\leq} \sqrt{\left(\mathrm{r}_{1}+\mathrm{r}_{2}\right)^{2}+\pi^{2} \mathrm{r}_{1} \mathrm{r}_{2}} \mathrm{~d}_{\mathfrak{C}}\left(\mathfrak{y}_{1}, \mathfrak{y}_{2}\right) \\
& \leq C_{*} \sqrt{\mathrm{r}_{1}^{2}+\mathrm{r}_{2}^{2}} \mathrm{~d}_{\mathfrak{C}}\left(\mathfrak{y}_{1}, \mathfrak{y}_{2}\right)
\end{aligned}
$$

Since the optimal plan $\alpha$ is concentrated on $\left\{r_{1}^{2}+r_{2}^{2} \leq R^{2}\right\}$ we obtain 


$$
\begin{aligned}
\left|\int_{X} \xi \mathrm{d}\left(\mu_{1}-\mu_{2}\right)\right| & =\left|\int \zeta\left(\mathfrak{y}_{1}\right)-\zeta\left(\mathfrak{y}_{2}\right) \mathrm{d} \boldsymbol{\alpha}\right| \leq \int\left|\zeta\left(\mathfrak{y}_{1}\right)-\zeta\left(\mathfrak{y}_{2}\right)\right| \mathrm{d} \boldsymbol{\alpha} \\
& \leq C_{*} R \int \mathrm{d}_{\mathfrak{C}}\left(\mathfrak{y}_{1}, \mathfrak{y}_{2}\right) \mathrm{d} \boldsymbol{\alpha} \leq C_{*} R \mathrm{H}\left(\mu_{1}, \mu_{2}\right) .
\end{aligned}
$$

\subsection{Hellinger-Kantorovich distance and Entropy-Transport functionals}

In this section we will establish our main result connecting the HellingerKantorovich Problem 7.4 defining HK with the Logarithmic Entropy-Transport Problem 6.1 defining LET.

It is clear that the definition of $\mathrm{HK}$ does not change if we replace the distance $\mathrm{d}$ on $X$ by its truncation $\mathrm{d}_{\pi}=\mathrm{d} \wedge \pi$. It is less obvious that we can even replace the threshold $\pi$ with $\pi / 2$ and use the distance $d_{\pi / 2, \mathfrak{C}}$ of Remark 7.2 in the formulation of the Hellinger-Kantorovich Problem 7.4. This property is related to the particular structure of the homogeneous marginals (which are not affected by masses concentrated in the vertex $\mathfrak{o}$ of the cone $\mathfrak{C}$ ); in [30, Sect.3.2] it is is called the presence of a sufficiently large reservoir, which shows that transport over distances larger than $\pi / 2$ is never optimal, since it is cheaper to transport into or out of the reservoir in $\mathfrak{o}$. This will provide an essential piece of information to connect the $\mathrm{HK}$ and the $L \mathbb{E}$ functionals.

In order to prove that transport only occurs of distances $\leq \pi / 2$ we define the subset

$$
\mathfrak{C}^{\prime}:=\left\{\mathrm{d}_{\pi / 2, \mathfrak{C}}<\mathrm{d}_{\mathfrak{C}}\right\}=\left\{\left(\mathfrak{y}_{1}, \mathfrak{y}_{2}\right) \in \mathfrak{C}_{\mathfrak{o}} \times \mathfrak{C}_{\mathfrak{o}}: \mathrm{d}\left(\mathrm{x}_{1}, \mathrm{x}_{2}\right)>\pi / 2\right\}
$$

and consider the partition $\left(\mathfrak{C}^{\prime}, \mathfrak{C}^{\prime \prime}\right)$ of $\mathfrak{C}=\mathfrak{C} \times \mathfrak{C}$, where $\mathfrak{C}^{\prime \prime}:=\mathfrak{C} \backslash \mathfrak{C}^{\prime}=$ $\left\{d_{\pi / 2, \mathfrak{C}}=d_{\mathfrak{C}}\right\}$. Observe that

$$
\mathfrak{C}_{\mathfrak{o}}^{\prime \prime}:=\mathfrak{C}^{\prime \prime} \cap\left(\mathfrak{C}_{\mathfrak{o}} \times \mathfrak{C}_{\mathfrak{o}}\right)=\left\{\left(\mathfrak{y}_{1}, \mathfrak{y}_{2}\right) \in \mathfrak{C}_{\mathfrak{o}} \times \mathfrak{C}_{\mathfrak{o}}: \mathrm{d}\left(\mathrm{x}_{1}, \mathrm{x}_{2}\right) \leq \pi / 2\right\} .
$$

In the following lemma we show that minimizers $\alpha \in \operatorname{Opt}_{\mathbb{K}}\left(\mu_{1}, \mu_{2}\right)$ are concentrated on $\mathfrak{C}^{\prime \prime}$, i.e. $\boldsymbol{\alpha}\left(\mathfrak{C}^{\prime}\right)=0$ which holds if and only if $\boldsymbol{\alpha}_{\mathfrak{o}}=\boldsymbol{\alpha}\left\llcorner\left(\mathfrak{C}_{\mathfrak{o}} \times\right.\right.$ $\mathfrak{C}_{\mathfrak{o}}$ ) is concentrated on $\mathfrak{C}_{\mathfrak{o}}^{\prime \prime}$. To handle the mass that is transported into or out of $\mathfrak{o}$, we use the continuous projections

$$
\mathfrak{g}_{i}: \mathfrak{C} \rightarrow \mathfrak{C}, \quad \mathfrak{g}_{1}\left(\mathfrak{y}_{1}, \mathfrak{y}_{2}\right):=\left(\mathfrak{y}_{1}, \mathfrak{o}\right), \quad \mathfrak{g}_{2}\left(\mathfrak{y}_{1}, \mathfrak{y}_{2}\right):=\left(\mathfrak{o}, \mathfrak{y}_{2}\right) .
$$

Lemma 7.19 (Plan restriction) For every $\alpha \in \mathcal{M}(\mathfrak{C})$ the plan

$$
\hat{\alpha}:=\boldsymbol{\alpha}^{\prime \prime}+\left(\mathfrak{g}_{1}\right)_{\sharp} \boldsymbol{\alpha}^{\prime}+\left(\mathfrak{g}_{2}\right)_{\sharp} \boldsymbol{\alpha}^{\prime} \quad \text { with } \quad \boldsymbol{\alpha}^{\prime}:=\boldsymbol{\alpha}\left\llcorner\mathfrak{C}^{\prime}, \quad \boldsymbol{\alpha}^{\prime \prime}:=\boldsymbol{\alpha}\left\llcorner\mathfrak{C}^{\prime \prime},\right.\right.
$$


is concentrated on $\mathfrak{C}^{\prime \prime}$, has the same homogeneous marginals as $\boldsymbol{\alpha}$, i.e. $\mathfrak{h}_{i}^{2} \hat{\boldsymbol{\alpha}}=$ $\mathfrak{h}_{i}^{2} \boldsymbol{\alpha}$, and

$$
\int_{\mathfrak{C}} d_{\mathfrak{C}}^{2} \mathrm{~d} \hat{\boldsymbol{\alpha}}=\int_{\mathfrak{C}} \mathrm{d}_{\pi / 2, \mathfrak{C}}^{2} \mathrm{~d} \hat{\boldsymbol{\alpha}} \leq \int_{\mathfrak{C}} \mathrm{d}_{\mathfrak{C}}^{2} \mathrm{~d} \boldsymbol{\alpha}
$$

where the inequality is strict if $\boldsymbol{\alpha}\left(\mathfrak{C}^{\prime}\right)>0$. In particular for every $\mu_{1}, \mu_{2} \in$ $\mathcal{M}(X)$

$$
\mathrm{HK}^{2}\left(\mu_{1}, \mu_{2}\right)=\min \left\{\int \mathrm{d}_{\pi / 2, \mathfrak{C}}^{2}\left(\mathfrak{y}_{1}, \mathfrak{y}_{2}\right) \mathrm{d} \boldsymbol{\alpha}: \boldsymbol{\alpha} \in \mathcal{M}_{2}(\mathfrak{C}), \mathfrak{h}_{i}^{2} \boldsymbol{\alpha}=\mu_{i}\right\} .
$$

Proof For every $\zeta \in \mathrm{B}_{b}(X)$, since $\mathrm{r}_{1} \circ \mathfrak{g}_{2}=0$ and $\mathrm{r}_{1} \circ \mathfrak{g}_{1}=\mathrm{r}_{1}$, we have

$$
\begin{aligned}
\int \zeta \mathrm{d}\left(\mathfrak{h}_{1}^{2} \hat{\boldsymbol{\alpha}}\right) & =\int \zeta\left(\mathbf{x}_{1}\right) \mathrm{r}_{1}^{2} \mathrm{~d} \hat{\boldsymbol{\alpha}}=\int \zeta\left(\mathbf{x}_{1}\right) \mathrm{r}_{1}^{2} \mathrm{~d} \boldsymbol{\alpha}^{\prime \prime}+\sum_{k} \int \zeta\left(\mathbf{x}_{1}\left(\mathfrak{g}_{k}\right)\right) \mathrm{r}_{1}\left(\mathfrak{g}_{k}\right)^{2} \mathrm{~d} \boldsymbol{\alpha}^{\prime} \\
& =\int \zeta\left(\mathbf{x}_{1}\right) \mathrm{r}_{1}^{2} \mathrm{~d} \boldsymbol{\alpha}^{\prime \prime}+\int \zeta\left(\mathbf{x}_{1}\right) \mathrm{r}_{1}^{2} \mathrm{~d} \boldsymbol{\alpha}^{\prime}=\int \zeta\left(\mathbf{x}_{1}\right) \mathrm{r}_{1}^{2} \mathrm{~d} \boldsymbol{\alpha}=\int \zeta \mathrm{d}\left(\mathfrak{h}_{1}^{2} \boldsymbol{\alpha}\right),
\end{aligned}
$$

so that $\mathfrak{h}_{1}^{2} \hat{\boldsymbol{\alpha}}=\mathfrak{h}_{1}^{2} \boldsymbol{\alpha}$; a similar calculation holds for $\mathfrak{h}_{2}^{2}$ so that $\hat{\boldsymbol{\alpha}} \in \mathfrak{H}_{=}^{2}\left(\mu_{1}, \mu_{2}\right)$. Moreover, if $\left(\mathfrak{y}_{1}, \mathfrak{y}_{2}\right) \in \mathfrak{C}^{\prime}$ we easily get

$$
d_{\mathfrak{C}^{\prime}}^{2}\left(\mathfrak{y}_{1}, \mathfrak{y}_{2}\right)>r_{1}^{2}+r_{2}^{2}=d_{\mathfrak{C}^{\prime}}^{2}\left(\mathfrak{g}_{1}\left(\mathfrak{y}_{1}, \mathfrak{y}_{2}\right)\right)+d_{\mathfrak{C}^{(}}^{2}\left(\mathfrak{g}_{2}\left(\mathfrak{y}_{1}, \mathfrak{y}_{2}\right)\right)
$$

so that whenever $\boldsymbol{\alpha}\left(\mathfrak{C}^{\prime}\right)>0$ we get

$$
\begin{aligned}
\int \mathrm{d}_{\mathfrak{C}}^{2} \mathrm{~d} \hat{\boldsymbol{\alpha}} & =\int\left(\mathrm{d}_{\mathfrak{C}}^{2} \circ \mathfrak{g}_{1}+\mathrm{d}_{\mathfrak{C}}^{2} \circ \mathfrak{g}_{2}\right) \mathrm{d} \boldsymbol{\alpha}^{\prime}+\int \mathrm{d}_{\mathfrak{C}}^{2} \mathrm{~d} \boldsymbol{\alpha}^{\prime \prime} \\
& <\int \mathrm{d}_{\mathfrak{C}}^{2} \mathrm{~d} \boldsymbol{\alpha}^{\prime}+\int \mathrm{d}_{\mathfrak{C}}^{2} \mathrm{~d} \boldsymbol{\alpha}^{\prime \prime}=\int \mathrm{d}_{\mathfrak{C}}^{2} \mathrm{~d} \boldsymbol{\alpha},
\end{aligned}
$$

which proves (7.53) and characterizes the equality case. (7.54) then follows by (7.53) and the fact that the homogeneous marginals of $\hat{\alpha}$ and $\alpha$ coincide.

In (7.54) we have established that $\boldsymbol{\alpha} \in \operatorname{Opt}_{\mathbb{K}_{K}}\left(\mu_{1}, \mu_{2}\right)$ has support in $\mathfrak{C}^{\prime \prime}$. This allows us to prove the identity $\mathbb{E T}=\mathrm{HK}^{2}$. For this, we introduce the open set $\mathfrak{G} \subset \mathfrak{C}^{\prime \prime}$ via

$$
\mathfrak{G}:=\left\{\left(\left[x_{1}, r_{1}\right],\left[x_{2}, r_{2}\right]\right) \in \mathfrak{C}_{\mathfrak{o}} \times \mathfrak{C}_{\mathfrak{o}}: \mathrm{d}\left(x_{1}, x_{2}\right)<\pi / 2\right\}
$$

and note that $r_{1} r_{2} \cos \left(d_{\pi / 2}\left(x_{1}, x_{2}\right)\right)>0$ in $\mathfrak{G}$. Recall also $\mathfrak{p}=\mathfrak{p} \otimes \mathfrak{p}: \boldsymbol{Y} \rightarrow \mathfrak{C}$, where $\mathfrak{p}$ is defined in (7.8). 
Theorem 7.20 $\left(\mathrm{H}^{2}=\mathrm{LT}\right)$ For all $\mu_{1}, \mu_{2} \in \mathcal{M}(X)$ we have

$$
\mathrm{H}^{2}\left(\mu_{1}, \mu_{2}\right)=\mathbb{E} \mathbb{T}\left(\mu_{1}, \mu_{2}\right),
$$

and $\boldsymbol{\alpha}\left(\mathfrak{C}^{\prime}\right)=0$ for optimal solution $\boldsymbol{\alpha} \in \mathcal{M}(\mathfrak{C})$ of Problem 7.4 or of $(7.32 \mathrm{a}$, b). Moreover,

(i) $\boldsymbol{\alpha} \in \mathcal{M}(\mathfrak{C})$ is an optimal plan for $(7.32 \mathrm{a}, \mathrm{b})$ if and only if $\boldsymbol{\alpha}\left(\mathfrak{C}^{\prime}\right)=0$ and $\mathbf{y}_{\sharp}\left(\boldsymbol{\alpha}\left\llcorner\mathfrak{C}_{\mathfrak{o}} \times \mathfrak{C}_{\mathfrak{o}}\right)\right.$ is an optimal plan for (6.33)-(6.32).

(ii) $\overline{\boldsymbol{\alpha}} \in \mathcal{M}(\boldsymbol{Y})$ is any optimal plan for (6.34) if and only if the plan $\hat{\boldsymbol{\alpha}}$ obtained from $\boldsymbol{\alpha}:=\mathfrak{p}_{\sharp} \overline{\boldsymbol{\alpha}}$ as in (7.52) is an optimal plan for the HellingerKantorovich Problem 7.4.

(iii) In the case that $\gamma \in \mathcal{M}(X \times X)$ belongs to $\operatorname{Opt}_{\text {ETT }}\left(\mu_{1}, \mu_{2}\right)$ and $\varrho_{i}: X \rightarrow[0, \infty)$ are Borel maps so that $\mu_{i}=\varrho_{i} \gamma_{i}+\mu_{i}^{\perp}$, then $\boldsymbol{\beta}:=\left(\mathfrak{p} \circ\left(x_{1}, \varrho_{1}^{1 / 2}\left(x_{1}\right) ; x_{2}, \varrho_{2}^{1 / 2}\left(x_{2}\right)\right)\right)_{\sharp} \boldsymbol{\gamma}$ is an optimal plan for $(7.32 \mathrm{a}, \mathrm{b})$, and it satisfies $\mathrm{r}_{1} \mathrm{r}_{2} \cos \left(\mathrm{d}_{\pi / 2}\left(\mathrm{x}_{1}, \mathrm{x}_{2}\right)\right)=1 \boldsymbol{\beta}$-a.e.; in particular $\boldsymbol{\beta}$ is concentrated on $\mathfrak{G}$.

(iv) If $\boldsymbol{\alpha} \in \mathcal{M}(\mathfrak{C})$ is an optimal plan for Problem 7.4 then $\tilde{\boldsymbol{\alpha}}:=\boldsymbol{\alpha}\llcorner\mathfrak{G}$ is an optimal plan for $(7.32 \mathrm{a}, \mathrm{b})$. Moreover,

- the plan $\boldsymbol{\beta}:=\operatorname{dil}_{\vartheta, 2}(\tilde{\boldsymbol{\alpha}})$, with $\vartheta:=\left(\mathrm{r}_{1} \mathrm{r}_{2} \cos \left(\mathrm{d}_{\pi / 2}\left(\mathbf{x}_{1}, \mathbf{x}_{2}\right)\right)\right)^{1 / 2}$, is an optimal plan for $(7.32 \mathrm{a}, \mathrm{b})$ satisfying $\mathrm{r}_{1} \mathrm{r}_{2} \cos \left(\mathrm{d}_{\pi / 2}\left(\mathrm{x}_{1}, \mathrm{x}_{2}\right)\right)=1 \boldsymbol{\beta}$-a.e.

- If $(X, \tau)$ is separable and metrizable, $\boldsymbol{\gamma}:=\left(\mathrm{x}_{1}, \mathrm{x}_{2}\right)_{\sharp} \boldsymbol{\beta}$ belongs to $\mathrm{Opt}_{\text {ET }}\left(\mu_{1}, \mu_{2}\right)$,

- If $(X, \tau)$ is separable and metrizable, $\boldsymbol{\beta}=\left(\mathfrak{p} \circ\left(x_{1}, \varrho_{1}^{1 / 2}\left(x_{1}\right) ; x_{2}, \varrho_{2}^{1 / 2}\right.\right.$ $\left.\left.\left(x_{2}\right)\right)\right)_{\sharp} \gamma$.

Proof Identity (7.55) and the first statement immediately follow by combining the previous Lemma 7.19 with Remark 7.5 and (6.34). Claim (ii) follows as well.

In order to prove (i), we observe that if $\alpha$ is an optimal plan for the formulation (7.32a,b) we can apply Lemma 7.9(iii) to find $\tilde{\alpha} \geq \boldsymbol{\alpha}$ optimal for (7.23), so that $\boldsymbol{\alpha}\left(\mathfrak{C}^{\prime}\right) \leq \tilde{\boldsymbol{\alpha}}\left(\mathfrak{C}^{\prime}\right)=0$. Given this property, (7.32a,b) correspond to $(6.33)-(6.32)$.

(iii) is a consequence of Theorem 6.7 and of the optimality conditions (6.19), which show that $\boldsymbol{\beta}$ is concentrated on $\mathfrak{G}$ and satisfies $r_{1} r_{2} \cos \left(\mathrm{d}_{\pi / 2}\left(\mathrm{x}_{1}, \mathrm{x}_{2}\right)\right)=$ $1 \boldsymbol{\beta}$-a.e. Therefore, $\boldsymbol{\beta}$ is optimal for (7.32a,b) thanks to claim (i).

Concerning (iv), the optimality of $\tilde{\alpha}$ is obvious from the formulation $(7.32 \mathrm{~b}$ ) and the optimality of $\boldsymbol{\beta}=\operatorname{dil}_{\vartheta, 2}(\tilde{\boldsymbol{\alpha}})$ follows from the invariance of (7.32b) with respect to dilations. We notice that $\boldsymbol{\beta}$-almost everywhere in $\mathfrak{G}$ we have

$$
\sum_{i} U_{0}\left(\mathrm{r}_{i}^{2}\right)+\mathrm{c}\left(\mathrm{x}_{1}, \mathrm{x}_{2}\right)=\sum_{i}\left(\mathrm{r}_{i}^{2}-1-\log \mathrm{r}_{i}^{2}\right)-\log \left(\cos ^{2}\left(\mathrm{~d}_{\pi / 2}\left(\mathrm{x}_{1}, \mathrm{x}_{2}\right)\right)\right)
$$




$$
\begin{aligned}
& =\sum_{i} r_{i}^{2}-2-2 \log \left(r_{1} r_{2} \cos \left(d_{\pi / 2}\left(x_{1}, x_{2}\right)\right)\right) \\
& =r_{1}^{2}+r_{2}^{2}-2 r_{1} r_{2} \cos \left(d_{\pi / 2}\left(x_{1}, x_{2}\right)\right)
\end{aligned}
$$

so that by $(7.32 \mathrm{a})$ we arrive at

$$
\int\left(\sum_{i} U_{0}\left(\mathrm{r}_{i}^{2}\right)+\mathrm{c}\left(\mathbf{x}_{1}, \mathbf{x}_{2}\right)\right) \mathrm{d} \boldsymbol{\beta}+\sum_{i}\left(\mu_{i}(X)-\mathfrak{h}_{i}^{2} \boldsymbol{\beta}(X)\right)=\mathrm{H}^{2}\left(\mu_{1}, \mu_{2}\right) .
$$

Let us now set $\boldsymbol{\gamma}:=\left(\mathrm{x}_{1}, \mathrm{x}_{2}\right)_{\sharp} \boldsymbol{\beta} \in \mathcal{M}(X \times X)$ and $\beta_{i}:=\pi_{\sharp}^{i} \boldsymbol{\beta} \in \mathcal{M}(\mathfrak{C})$, which yield $\gamma_{i}:=\pi_{\sharp}^{i} \boldsymbol{\gamma}=\left(\mathbf{x}_{i}\right)_{\sharp} \boldsymbol{\beta}=\mathbf{x}_{\sharp} \beta_{i} \in \mathcal{M}(X)$ and $\tilde{\mu}_{i}:=\mathfrak{h}_{i}^{2} \boldsymbol{\beta}=\left(\mathbf{x}_{i}\right)_{\sharp}\left(r_{i}^{2} \boldsymbol{\gamma}\right)=$ $\mathbf{X}_{\sharp}\left(r^{2} \beta_{i}\right)$. Denoting by $\left(\beta_{i, x_{i}}\right)_{x_{i} \in X}$ the disintegration of $\beta_{i}$ with respect to $\gamma_{i}$ (here we need the metrizability and separability of $(X, \tau)$, see [2, Sect. 5.3]), we find

$$
\begin{aligned}
\int_{X} \zeta \mathrm{d} \tilde{\mu}_{i} & =\int_{\mathfrak{C}} \zeta(\mathbf{x}) r^{2} \mathrm{~d} \beta_{i}=\int_{X}\left(\int_{\mathfrak{C}} \zeta(\mathbf{x}) r^{2} \mathrm{~d} \beta_{i, x}\right) \mathrm{d} \gamma_{i} \\
& =\int_{X} \zeta(x)\left(\int_{\mathfrak{C}} r^{2} \mathrm{~d} \beta_{i, x}\right) \mathrm{d} \gamma_{i}
\end{aligned}
$$

for all $\zeta \in \mathrm{B}_{b}(X)$, so that

$$
\tilde{\mu}_{i}=\tilde{\varrho}_{i} \gamma_{i} \leq \mu_{i} \text { with } \tilde{\varrho}_{i}(x):=\int_{\mathfrak{C}} r^{2} \mathrm{~d} \beta_{i, x}
$$

Applying Jensen's inequality we obtain

$$
\begin{aligned}
\int U_{0}\left(r_{i}^{2}\right) \mathrm{d} \boldsymbol{\beta} & =\int U_{0}\left(\mathrm{r}_{i}^{2}\right) \mathrm{d} \beta_{i}=\int\left(\int U_{0}\left(r_{i}^{2}\right) \mathrm{d} \beta_{i, x_{i}}\left(r_{i}\right)\right) \mathrm{d} \gamma_{i} \\
& \geq \int U_{0}\left(\int r_{i}^{2} \mathrm{~d} \beta_{i, x_{i}}\left(r_{i}\right)\right) \mathrm{d} \gamma_{i}=\int U_{0}\left(\tilde{\varrho}_{i}(x)\right) \mathrm{d} \gamma_{i} .
\end{aligned}
$$

Now $\int \mathrm{c}\left(\mathbf{x}_{1}, \mathbf{x}_{2}\right) \mathrm{d} \boldsymbol{\beta}=\int \mathrm{c}\left(x_{1}, x_{2}\right) \mathrm{d} \boldsymbol{\gamma}$ and (7.56) imply

$$
\mathrm{H}^{2}\left(\mu_{1}, \mu_{2}\right) \geq \sum_{i} \int_{X} U_{0}\left(\tilde{\varrho}_{i}\right) \mathrm{d} \gamma_{i}+\int_{X \times X} \mathrm{~cd} \boldsymbol{\gamma}+\sum_{i} v_{i}(X)
$$

with $\nu_{i}:=\mu_{i}-\tilde{\mu}_{i} \in \mathcal{M}(X)$. Hence, since $\mu_{i}=\tilde{\varrho}_{i} \gamma_{i}+v_{i}$ and the standard decomposition $\mu_{i}=\varrho_{i} \gamma_{i}+\mu_{i}^{\perp}\left(\right.$ cf. (2.8)) give $v_{i}=\mu_{i}^{\perp}+\left(\varrho_{i}-\tilde{\varrho}_{i}\right) \gamma_{i} \geq \mu_{i}^{\perp}$, 
$U_{0}(s)=s-1-\log s$ and the monotonicity of the logarithm yield

$$
\begin{aligned}
\mathrm{H}^{2}\left(\mu_{1}, \mu_{2}\right) & \geq \sum_{i}\left(\int_{X} U_{0}\left(\tilde{\varrho}_{i}\right) \mathrm{d} \gamma_{i}+v_{i}(X)\right)+\int \mathrm{c} \mathrm{d} \gamma \\
& =\sum_{i}\left(\int_{X}\left(U_{0}\left(\tilde{\varrho}_{i}\right)+\varrho_{i}-\tilde{\varrho}_{i}\right) \mathrm{d} \gamma_{i}+\mu_{i}^{\perp}(X)\right)+\int \mathrm{c} \mathrm{d} \boldsymbol{\gamma} \\
& \geq \sum_{i}\left(\int_{X} U_{0}\left(\varrho_{i}\right) \mathrm{d} \gamma_{i}+\mu_{i}^{\perp}(X)\right)+\int \mathrm{cd} \boldsymbol{\gamma} \geq \operatorname{LT}\left(\mu_{1}, \mu_{2}\right),
\end{aligned}
$$

where the last estimate follows from Theorem 6.2(b). Above, the first inequality is strict if $v_{i} \neq \mu_{i}^{\perp}$ so that $\varrho_{i}>\tilde{\varrho}_{i}$ on some set with positive $\gamma_{i}$-measure.

By the first statement of the Theorem it follows that $\gamma \in \mathrm{Opt}_{\text {ET }}\left(\mu_{1}, \mu_{2}\right)$. Hence, all the inequalities are in fact identities, and we conclude $\tilde{\varrho}_{i} \equiv \varrho_{i}$. Since $U_{0}$ is strictly convex, the disintegration measure $\beta_{i, x_{i}}$ is a Dirac measure concentrated on $\sqrt{\varrho_{i}\left(x_{i}\right)}$, so that $\boldsymbol{\beta}=\left(\mathfrak{p} \circ\left(x_{1}, \varrho_{1}^{1 / 2}\left(x_{1}\right) ; x_{2}, \varrho_{2}^{1 / 2}\left(x_{2}\right)\right)\right)_{\sharp} \boldsymbol{\gamma}$.

We observe that the system $\left(\boldsymbol{\gamma}, \varrho_{1}, \varrho_{2}\right)$ provided by the previous Theorem enjoys a few remarkable properties, that are not obvious from the original Hellinger-Kantorovich formulation.

(a) First of all, thanks to (6.15), the annihilated part $\mu_{i}^{\perp}$ of the measures $\mu_{i}$ is concentrated on the set

$$
M_{i, j}:=\left\{x_{i} \in X: \mathrm{d}\left(x_{i}, \operatorname{supp}\left(\mu_{j}\right)\right) \geq \pi / 2\right\}
$$

When $\mu_{i}\left(M_{i, j}\right)=0$ then $\mu_{i} \ll \gamma_{i}$.

(b) As a second property, an optimal plan $\gamma \in \operatorname{Opt}_{\text {EET }}\left(\mu_{1}, \mu_{2}\right)$ provides an optimal plan $\boldsymbol{\alpha}=\left(\mathfrak{p} \circ\left(x_{1}, \varrho_{1}^{1 / 2}\left(x_{1}\right) ; x_{2}, \varrho_{2}^{1 / 2}\left(x_{2}\right)\right)\right)_{\sharp} \boldsymbol{\gamma}$ which is concentrated on the graph of the map $\left(\varrho_{1}^{1 / 2}\left(x_{1}\right) ; \varrho_{2}^{1 / 2}\left(x_{2}\right)\right)$ from $X \times X$ to $\mathbb{R}_{+} \times \mathbb{R}_{+}$, where the maps $\varrho_{i}$ are independent, in the sense that $\varrho_{i}$ only depends on $x_{i}$.

(c) A third important application of Theorem 7.20 is the duality formula for the $\mathrm{HK}$ functional which directly follows from (6.14) of Theorem 6.3. We will state it in a slightly different form in the next theorem, whose interpretation will be clearer in the light of Sect. 8.4. It is based on the inf-convolution formula

$$
\begin{aligned}
\mathscr{P}_{1} \xi(x) & =\inf _{x^{\prime} \in X}\left(\frac{\xi\left(x^{\prime}\right)}{1+2 \xi\left(x^{\prime}\right)}+\frac{\sin ^{2}\left(\mathrm{~d}_{\pi / 2}\left(x, x^{\prime}\right)\right)}{2\left(1+2 \xi\left(x^{\prime}\right)\right)}\right) \\
& =\inf _{x^{\prime} \in X} \frac{1}{2}\left(1-\frac{\cos ^{2}\left(\mathrm{~d}_{\pi / 2}\left(x, x^{\prime}\right)\right)}{1+2 \xi\left(x^{\prime}\right)}\right) .
\end{aligned}
$$


where $\xi \in \mathrm{B}(X)$ with $\xi>-1 / 2$.

Theorem 7.21 (Duality formula for $\mathrm{HK}$ )

(i) If $\xi \in \mathrm{B}_{b}(X)$ with $\inf _{X} \xi>-1 / 2$ then the function $\mathscr{P}_{1} \xi$ defined by (7.57) belongs to $\operatorname{Lip}_{b}(X)$, satisfies $\sup _{X} \mathscr{P}_{1} \xi<1 / 2$, and admits the equivalent representation

$$
\mathscr{P}_{1} \xi(x)=\inf _{x^{\prime} \in B_{\pi / 2}(x)} \frac{1}{2}\left(1-\frac{\cos ^{2}\left(\mathrm{~d}_{\pi / 2}\left(x, x^{\prime}\right)\right)}{1+2 \xi\left(x^{\prime}\right)}\right) .
$$

In particular, if $\xi$ has bounded support then $\mathscr{P}_{1} \xi \in \operatorname{Lip}_{b s}(X)$, the space of Lipschitz functions with bounded support.

(ii) Let us suppose that $(X, \mathrm{~d})$ is a separable metric space and $\tau$ is induced by $\mathrm{d}$. For every $\mu_{0}, \mu_{1} \in \mathcal{M}(X)$ we have

$$
\begin{aligned}
& \frac{1}{2} \mathrm{~K}^{2}\left(\mu_{0}, \mu_{1}\right)=\sup \left\{\int \mathscr{P}_{1} \xi \mathrm{d} \mu_{1}-\int \xi \mathrm{d} \mu_{0}:\right. \\
& \left.\quad \xi \in \operatorname{Lip}_{b s}(X), \inf _{X} \xi>-1 / 2\right\} .
\end{aligned}
$$

Proof Let us first observe that if

$$
-\frac{1}{2}<a \leq \xi \leq b \text { in } X \Rightarrow \frac{a}{1+2 a} \leq \mathscr{P}_{1} \xi \leq \frac{b}{1+2 b} \text { in } X,
$$

where the upper bound follows using $x^{\prime}=x$, while the lower bound is easily seen from the first form of $\mathscr{P}_{1} \xi$ in $(7.57)$ and $\sin ^{2} \geq 0$. Since $1 /\left(1+2 \xi\left(x^{\prime}\right)\right) \leq$ $1 /(1+2 a)$ for every $x^{\prime} \in X$, the function $\mathscr{P}_{1} \xi$ is also Lipschitz, because it is the infimum of a family of uniformly Lipschitz functions.

Moreover we have the estimate

$$
\frac{1}{2}\left(1-\frac{\cos ^{2}\left(\mathrm{~d}_{\pi / 2}\left(x, x^{\prime}\right)\right)}{1+2 \xi\left(x^{\prime}\right)}\right)=\frac{1}{2}>\frac{b}{1+2 b} \quad \text { if } \mathrm{d}\left(x, x^{\prime}\right) \geq \pi / 2,
$$

which immediately gives (7.58). In particular, we have

$$
\xi \equiv 0 \text { in } X \backslash B \Rightarrow \mathscr{P}_{1} \xi \equiv 0 \text { in }\{x \in X: \mathrm{d}(x, B) \geq \pi / 2\} .
$$

Let us now prove statement (ii). We denote by $E$ the the right-hand side of (7.59) and by $E^{\prime}$ the analogous expression where $\xi$ runs in $\mathrm{C}_{b}(X)$ :

$$
E^{\prime}:=\sup \left\{\int \mathscr{P}_{1} \xi \mathrm{d} \mu_{1}-\int \xi \mathrm{d} \mu_{0}: \xi \in \mathrm{C}_{b}(X), \inf _{X} \xi>-1 / 2\right\} .
$$


It is clear that $E^{\prime} \geq E$. If $\xi \in \mathrm{C}_{b}(X)$ with inf $\xi>-1 / 2$, setting $\psi_{1}\left(x_{1}\right):=$ $-2 \xi\left(x_{1}\right), \psi_{2}\left(x_{2}\right):=2\left(\mathscr{P}_{1} \xi\right)\left(x_{2}\right)$, we know that $\sup _{X} \psi_{2}<1$ and $\psi_{2} \in$ $\operatorname{Lip}_{b}(X)$. Thus, $\psi_{1}$ and $\psi_{2}$ are continuous and satisfy

$$
\left(1-\psi_{2}\left(x_{2}\right)\right)\left(1-\psi_{1}\left(x_{1}\right)\right) \geq \cos ^{2}\left(\mathrm{~d}_{\pi / 2}\left(x_{1}, x_{2}\right)\right) .
$$

Hence, the pair $\left(\psi_{1}, \psi_{2}\right)$ is admissible for (6.14) (with $\mathrm{C}_{b}(X)$ instead of $\operatorname{LSC}_{s}(X)$; note that $\tau$ is metrizable and thus completely regular), so that $\mathrm{HK}^{2}\left(\mu_{0}, \mu_{1}\right)=\operatorname{LET}\left(\mu_{0}, \mu_{1}\right) \geq E^{\prime}$.

On the other hand, if $\left(\psi_{1}, \psi_{2}\right) \in \mathrm{C}_{b}(X) \times \mathrm{C}_{b}(X)$ with $\sup _{X} \psi_{i}<1$, setting $\xi_{1}=-\frac{1}{2} \psi_{1}$ and $\tilde{\xi}_{2}:=\mathscr{P}_{1}\left(-\xi_{1}\right)$ we see that $2 \tilde{\xi}_{2} \geq \psi_{2}$ giving $E^{\prime} \geq$ $\mathrm{HK}^{2}\left(\mu_{0}, \mu_{1}\right)$, so that $\mathrm{H}^{2}\left(\mu_{0}, \mu_{1}\right)=E^{\prime}$.

It remains to show that $E \geq E^{\prime}$. We first approximate $\xi \in \mathrm{C}_{b}(X)$ with $\inf _{X} \xi>-1 / 2$ by a decreasing sequence of Lipschitz and bounded functions (e.g. by taking $\left.\xi_{n}(x):=\sup _{y} \xi(y)-n \mathrm{~d}_{\pi}(x, y)\right)$ pointwise converging to $\xi$, observing that $\mathscr{P}_{1} \xi_{n}$ is also decreasing, uniformly bounded and pointwise converging to $\mathscr{P}_{1} \xi$. We deduce that the supremum in (7.63) does not change if we restrict it to $\operatorname{Lip}_{b}(X)$.

In the last step of the proof we want to show that we can eventually restrict the supremum in (7.63) to $\operatorname{Lip}_{b s}(X)$, by a further approximation argument. We fix a Lipschitz function $\xi$ valued in $[a, b]$ with $-1 / 2<a \leq 0 \leq b$ and we consider the increasing sequence of nonnegative cut-off functions $\zeta_{n}(x):=$ $0 \vee(n-\mathrm{d}(x, \bar{x})) \wedge 1$ : they are uniformly 1-Lipschitz, have bounded support and satisfy $\zeta_{n} \uparrow 1$ as $n \rightarrow \infty$. It is easy to check that $\xi_{n}:=\zeta_{n} \xi$ belong to $\operatorname{Lip}_{b s}(X)$ and take values in the interval $[a, b]$ so that $\frac{a}{1+2 a} \leq \mathscr{P}_{1} \xi_{n} \leq \frac{b}{1+2 b}$ for every $n \in \mathbb{N}$ by (7.60).

Since $\xi_{n}(x)=0$ if $\mathrm{d}(x, \bar{x}) \geq n$ and $\xi_{n}(x)=\xi(x)$ if $\mathrm{d}(x, \bar{x}) \leq n-1$, by (7.58) we get

$$
\begin{aligned}
& \mathscr{P}_{1} \xi_{n}(x)=0 \quad \text { if } \mathrm{d}(x, \bar{x}) \geq n+\pi / 2, \\
& \mathscr{P}_{1} \xi_{n}(x)=\mathscr{P}_{1} \xi(x) \text { if } \mathrm{d}(x, \bar{x})<n-1-\pi / 2
\end{aligned}
$$

Thus $\mathscr{P}_{1} \xi_{n} \in \operatorname{Lip}_{b s}(X)$ and $\mathscr{P}_{1} \xi_{n}(x) \rightarrow \mathscr{P}_{1} \xi(x)$ for every $x \in X$ as $n \rightarrow \infty$. Applying the Lebesgue Dominated Convergence theorem we conclude that

$$
\lim _{n \rightarrow \infty} \int_{X} \mathscr{P}_{1} \xi_{n} \mathrm{~d} \mu_{1}-\int_{X} \xi_{n} \mathrm{~d} \mu_{0}=\int_{X} \mathscr{P}_{1} \xi \mathrm{d} \mu_{1}-\int_{X} \xi \mathrm{d} \mu_{0}
$$




\subsection{Limiting cases: recovering the Hellinger-Kakutani distance and the Kantorovich-Wasserstein distance}

In this section we will show that we can recover the Hellinger-Kakutani and the Kantorovich-Wasserstein distance by suitably rescaling the $\mathrm{H}$ functional. The Hellinger-Kakutani distance. As we have seen in Example E.5 of Sect. 3.3, the Hellinger-Kakutani distance between two measures $\mu_{1}, \mu_{2} \in$ $\mathcal{M}(X)$ can be obtained as a limiting case when the space $X$ is endowed with the discrete distance

$$
\mathrm{d}_{\mathrm{He}}\left(x_{1}, x_{2}\right):=\left\{\begin{array}{ll}
a & \text { if } x_{1} \neq x_{2} \\
0 & \text { if } x_{1}=x_{2},
\end{array} \quad \text { with } a \in[\pi, \infty] .\right.
$$

The induced cone distance in this case is

$$
\mathrm{d}_{\mathfrak{C}}^{2}\left(\left[x_{1}, r_{1}\right],\left[x_{2}, r_{2}\right]\right)= \begin{cases}\left(r_{1}-r_{2}\right)^{2} & \text { if } x_{1}=x_{2}, \\ \left(r_{1}+r_{2}\right)^{2} & \text { if } x_{1} \neq x_{2} .\end{cases}
$$

and the induced cost function for the Entropy-Transport formalism is given by

$$
\mathrm{C}_{\mathrm{He}}\left(x_{1}, x_{2}\right):= \begin{cases}0 & \text { if } x_{1}=x_{2} \\ +\infty & \text { otherwise }\end{cases}
$$

Recalling (3.21)-(3.22) we obtain

$$
\begin{aligned}
\operatorname{He}^{2}\left(\mu_{1}, \mu_{2}\right) & =\operatorname{LET}_{\mathrm{He}}\left(\mu_{1}, \mu_{2}\right)=\int_{X}\left(\sqrt{\varrho_{1}}-\sqrt{\varrho_{2}}\right)^{2} \mathrm{~d} \gamma \\
\text { with } \mu_{i} & =\varrho_{i} \gamma \ll \gamma \in \mathcal{M}(X) .
\end{aligned}
$$

Since $\mathrm{C}_{\mathrm{He}} \geq \mathrm{C}=\ell(\mathrm{d})$ for every distance function on $X$, we always have the upper bound

$$
\mathrm{H}\left(\mu_{1}, \mu_{2}\right) \leq \mathrm{He}\left(\mu_{1}, \mu_{2}\right) \text { for every } \mu_{1}, \mu_{2} \in \mathcal{M}(X) .
$$

Applying Lemma 3.9 we easily get

Theorem 7.22 (Convergence of $\mathrm{HK}$ to $\mathrm{He}$ ) Let $(X, \tau, \mathrm{d})$ be an extended metric topological space and let $\mathrm{H}_{\lambda \mathrm{d}}$ be the Hellinger-Kantorovich distances in $\mathcal{M}(X)$ induced by the distances $\mathrm{d}_{\lambda}:=\lambda \mathrm{d}, \lambda>0$. For every pair $\mu_{1}, \mu_{2} \in$ $\mathcal{M}(X)$ we have

$$
\mathrm{HK}_{\lambda \mathrm{d}}\left(\mu_{1}, \mu_{2}\right) \uparrow \mathrm{He}\left(\mu_{1}, \mu_{2}\right) \text { as } \lambda \uparrow \infty \text {. }
$$


The Kantorovich-Wasserstein distance. Let us first observe that whenever $\mu_{1}, \mu_{2} \in \mathcal{M}(X)$ have the same mass their $\mathrm{H}$-distance is always bounded form above by the Kantorovich-Wasserstein distance $\mathrm{W}_{\mathrm{d}}$ (the upper bound is trivial when $\mu_{1}(X) \neq \mu_{2}(X)$, since in this case $\left.W_{d}\left(\mu_{1}, \mu_{2}\right)=+\infty\right)$.

Proposition 7.23 For every pair $\mu_{1}, \mu_{2} \in \mathcal{M}(X)$ we have

$$
\mathrm{H}\left(\mu_{1}, \mu_{2}\right) \leq \mathrm{W}_{\mathrm{d}_{\pi / 2}}\left(\mu_{1}, \mu_{2}\right) \leq \mathrm{W}_{\mathrm{d}}\left(\mu_{1}, \mu_{2}\right) .
$$

Proof It is not restrictive to assume that $\mathrm{W}_{\mathrm{d}_{\pi / 2}}^{2}\left(\mu_{1}, \mu_{2}\right)=\int \mathrm{d}_{\pi / 2}^{2} \gamma<\infty$ for an optimal plan $\boldsymbol{\gamma}$ with marginals $\mu_{i}$. We then define the plan $\boldsymbol{\alpha}:=\mathfrak{s}_{\sharp} \boldsymbol{\gamma} \in$ $\mathcal{M}(\mathfrak{C} \times \mathfrak{C})$ where $\mathfrak{s}\left(x_{1}, x_{2}\right):=\left(\left[x_{1}, 1\right],\left[x_{2}, 1\right]\right)$, so that $\mathfrak{h}_{i}^{2} \boldsymbol{\alpha}=\mu_{i}$. By using (7.54) and the identity $2-2 \cos ^{2}(d)=4 \sin ^{2}(d / 2)$ we obtain

$$
\begin{aligned}
\mathrm{HK}^{2}\left(\mu_{1}, \mu_{2}\right) & \leq 4 \int_{\mathfrak{C}} \sin ^{2}\left(\mathrm{~d}_{\pi / 2}\left(\mathbf{x}_{1}, \mathbf{x}_{2}\right) / 2\right) \mathrm{d} \boldsymbol{\alpha} \leq \int_{\boldsymbol{X}} \mathrm{d}_{\pi / 2}^{2}\left(x_{1}, x_{2}\right) \mathrm{d} \boldsymbol{\gamma} \\
& \leq \mathbf{W}_{\mathrm{d}_{\pi / 2}}^{2}\left(\mu_{1}, \mu_{2}\right) .
\end{aligned}
$$

In order to recover the Kantorovich-Wasserstein distance we perform a simultaneous scaling, by taking the limit of $n \mathrm{HK}_{\mathrm{d} / n}$ where $\mathrm{HK}_{\mathrm{d} / n}$ is induced by the distance $\mathrm{d} / n$.

Theorem 7.24 (Convergence of $\mathrm{HK}$ to $\mathrm{W})$ Let $(X, \tau, \mathrm{d})$ be an extended metric topological space and let $\mathrm{H}_{\mathrm{d} / \lambda}$ be the Hellinger-Kantorovich distances in $\mathcal{M}(X)$ induced by the distances $\lambda^{-1} \mathrm{~d}$ for $\lambda>0$. Then, for all $\mu_{1}, \mu_{2} \in \mathcal{M}(X)$ we have

$$
\lambda \mathrm{H}_{\mathrm{d} / \lambda}\left(\mu_{1}, \mu_{2}\right) \uparrow \mathrm{W}_{\mathrm{d}}\left(\mu_{1}, \mu_{2}\right) \text { as } \lambda \uparrow \infty \text {. }
$$

Proof Let us denote by $\mathrm{LT}_{\lambda}=\mathrm{H}_{\mathrm{d} / \lambda}^{2}$ the optimal value of the LET-problem associated to $d / \lambda$. Since the Kantorovich-Wasserstein distance is invariant by the rescaling $\lambda \mathrm{W}_{\mathrm{d} / \lambda}=\mathrm{W}_{\mathrm{d}}$, estimate (7.71) shows that $\lambda \mathrm{H}_{\mathrm{d} / \lambda} \leq \mathrm{W}_{\mathrm{d}}$.

As $x \mapsto \sin (x \wedge \pi / 2)$ is concave in $[0, \infty)$, the function $x \mapsto \sin (x \wedge \pi / 2) / x$ is decreasing in $[0, \infty)$, so that $\alpha \sin ((d / \alpha) \wedge \pi / 2) \leq \lambda \sin ((d / \lambda) \wedge \pi / 2)$ for every $d \geq 0$ and $0<\alpha<\lambda$. Combining (7.54) with (7.11b) we see that the map $\lambda \mapsto \lambda \mathrm{H}_{\mathrm{d} / \lambda}\left(\mu_{1}, \mu_{2}\right)$ is nondecreasing.

It remains to prove that $L:=\lim _{\lambda \rightarrow \infty} \lambda \mathrm{HK}_{\mathrm{d} / \lambda}\left(\mu_{1}, \mu_{2}\right)=\sup _{\lambda \geq 1} \lambda \mathrm{H}_{\mathrm{d} / \lambda}$ $\left(\mu_{1}, \mu_{2}\right) \geq \mathbf{W}_{\mathrm{d}}\left(\mu_{1}, \mu_{2}\right)$. For this, it is not restrictive to assume that $L$ is finite.

Let $\boldsymbol{\gamma}_{\lambda}$ be an optimal plan for $\mathrm{H}_{\mathrm{d} / \lambda}\left(\mu_{1}, \mu_{2}\right)$ with marginals $\gamma_{\lambda, i}=\pi_{\sharp}^{i} \boldsymbol{\gamma}_{\lambda}$. We denote by $\mathscr{F}$ the entropy functionals associated to logarithmic entropy $U_{1}(s)$ and by $\mathscr{G}$ the entropy functionals associated to $\mathrm{I}_{1}(s)$ as in Example E.3 
of Sect. 3.3. Since the transport part of the LET-functional is associated to the costs

$$
\mathrm{c}_{\lambda}\left(x_{1}, x_{2}\right)=\lambda^{2} \ell\left(\mathrm{d}\left(x_{1}, x_{2}\right) / \lambda\right) \stackrel{(6.6)}{\geq} \mathrm{d}^{2}\left(x_{1}, x_{2}\right)
$$

we obtain the estimate

$$
L^{2} \geq \lambda^{2} \operatorname{LT}_{\lambda}\left(\mu_{1}, \mu_{2}\right) \geq \sum_{i} \lambda^{2} \mathscr{F}\left(\gamma_{\lambda, i} \mid \mu_{i}\right)+\int_{X} \mathrm{~d}^{2}\left(x_{1}, x_{2}\right) \mathrm{d} \boldsymbol{\gamma}_{\lambda} .
$$

Proposition 2.10 shows that the family of plans $\left(\boldsymbol{\gamma}_{\lambda}\right)_{\lambda \geq 1}$ is relatively compact with respect to narrow convergence in $\mathcal{M}(X \times X)$. Since $\lambda^{2} F(s) \uparrow \mathrm{I}_{1}(s)$, passing to the limit along a suitable subnet $(\lambda(\alpha))_{\alpha \in \mathbb{A}}$ parametrized by a directed set $\mathbb{A}$, and applying Corollary 2.9 we get a limit plan $\gamma \in \mathcal{M}(X \times X)$ with marginals $\gamma_{i}$ such that

$$
\sum_{i} \mathscr{G}\left(\gamma_{i} \mid \mu_{i}\right) \leq L^{2}, \quad \text { which implies } \quad \gamma_{i}=\mu_{i}
$$

In particular, we conclude that $\mu_{1}(X)=\boldsymbol{\gamma}(X \times X)=\mu_{2}(X)$. Since $\mathrm{d}$ is lower semicontinuous, narrow convergence of $\boldsymbol{\gamma}_{\lambda(\alpha)}$ and (7.73) also yield

$$
L^{2} \geq \liminf _{\alpha \in \mathbb{A}} \int_{\boldsymbol{X}} \mathrm{d}^{2}\left(x_{1}, x_{2}\right) \mathrm{d} \boldsymbol{\gamma}_{\lambda(\alpha)} \geq \int_{\boldsymbol{X}} \mathrm{d}^{2}\left(x_{1}, x_{2}\right) \mathrm{d} \boldsymbol{\gamma} \geq \mathrm{W}_{\mathrm{d}}^{2}\left(\mu_{1}, \mu_{2}\right) .
$$

\subsection{The Gaussian Hellinger-Kantorovich distance}

We conclude this general introduction to the Hellinger-Kantorovich distance by discussing another interesting example.

We consider the inverse function $g: \mathbb{R}_{+} \rightarrow[0, \pi / 2)$ of $\sqrt{\ell}$ :

$$
g(z):=\arccos \left(\mathrm{e}^{-z^{2} / 2}\right), \text { giving } g(0)=0, g^{\prime}(0)=1, \ell(g(d))=d^{2} .
$$

Since $\sqrt{\ell}$ is a convex function, $g$ is a concave increasing function in $[0, \infty)$ with $g(z) \leq z$ and $\lim _{z \rightarrow \infty} g(z)=\pi / 2$.

It follows that $\mathrm{g}:=g \circ \mathrm{d}$ is a distance in $X$, inducing the same topology as d. We can now introduce a distance $\mathrm{H}_{\mathrm{g}}$ associated to $\mathrm{g}$. The corresponding distance on $\mathfrak{C}^{2}$ is given by

$$
g_{\mathfrak{c}}\left(\mathfrak{y}_{1}, \mathfrak{y}_{2}\right):=r_{1}^{2}+r_{2}^{2}-2 r_{1} r_{2} \exp \left(-d^{2}\left(x_{1}, x_{2}\right) / 2\right)
$$


From $g(z) \leq z$ we have $\mathrm{g}_{\mathfrak{C}} \leq \mathrm{d}_{\mathfrak{C}}$.

We can then apply Corollary 7.14, Theorems 7.15, 7.17, 7.20, and 6.3 to obtain the following result.

Theorem 7.25 (The Gaussian Hellinger-Kantorovich distance) The functional

$$
\begin{aligned}
& \mathbf{G K}^{2}\left(\mu_{1}, \mu_{2}\right):=\mathrm{HK}_{\mathrm{g}}^{2}\left(\mu_{1}, \mu_{2}\right) \\
& =\min \left\{\int \mathfrak{g}_{\mathfrak{C}}^{2}\left(\mathfrak{y}_{1}, \mathfrak{y}_{2}\right) \mathrm{d} \boldsymbol{\alpha}: \boldsymbol{\alpha} \in \mathcal{M}(\mathfrak{C}), \mathfrak{h}_{i}^{2} \boldsymbol{\alpha}=\mu_{i}\right\}
\end{aligned}
$$

defines a distance on $\mathcal{M}(X)$ dominated by $\mathrm{H}$. If $(X, \mathrm{~d})$ is separable (resp. complete) then ( $\mathcal{M}(X), \mathrm{GK})$ is a separable (resp. complete) metric space, whose topology coincides with the weak convergence. We also have

$$
\begin{aligned}
\operatorname{GK}^{2}\left(\mu_{1}, \mu_{2}\right) & =\min \left\{\sum_{i} \mathscr{F}\left(\gamma_{i} \mid \mu_{i}\right)+\int_{\boldsymbol{X}} \mathrm{d}^{2}\left(x_{1}, x_{2}\right) \mathrm{d} \boldsymbol{\gamma}: \boldsymbol{\gamma} \in \mathcal{M}(\boldsymbol{X})\right\} \\
& =\sup \left\{\sum_{i} \int\left(1-\mathrm{e}^{-\varphi_{i}}\right) \mathrm{d} \mu_{i}: \varphi_{1} \oplus \varphi_{2} \leq \mathrm{d}^{2}\right\} .
\end{aligned}
$$

We shall see in the next Sect. 8.2 that $\mathrm{HK}$ is the length distance induced by $\mathrm{GK}$ if $\mathrm{d}$ is a length distance on $X$.

\section{Dynamic interpretation of the Hellinger-Kantorovich distance}

In this section we collect our main results concerning the dynamic interpretation of the Hellinger-Kantorovich distance: it reveals another deep connection with Optimal Transport problems, in particular as a natural generalization of the Benamou-Brenier [7] characterization of the Kantorovich-Wasserstein distance, see the next Sect. 8.4 and [30, Sect.4], where a more direct approach has been adopted for the case $X=\mathbb{R}^{d}$.

In order to deal with arbitrary geodesic spaces $X$ and to obtain other important results concerning general representation formulae for geodesics and absolutely continuous curves (Sect. 8.2), lower curvature bounds (Sect. 8.3), duality relations with subsolutions to Hamilton-Jacobi equations (Sects. 8.4 and 8.6), and contraction properties for diffusion semigroups (Sect. 8.7), we adopted here the point of view of dynamic plans (i.e. probability measures on continuous paths), which provide a powerful tool in Optimal Transport, cf. [2, Chap. 8]. It is not difficult to imagine that the natural objects to deal with the Hellinger-Kantorovich distance are dynamic plans in the cone $\mathfrak{C}$, so we will devote the next section to recall the basic metric properties of curves in $\mathfrak{C}$. 
As in Sect. 7.5, in all this section we will suppose that $(X, \mathrm{~d})$ is a complete and separable (possibly extended) metric space and $\tau$ coincides with the topology induced by $\mathrm{d}$. All the results admit a natural generalization to the framework of extended metric-topological spaces [1, Sect.4].

\subsection{Absolutely continuous curves and geodesics in the cone $\mathfrak{C}$}

Absolutely continuous curves and metric derivative. If $\left(Z, d_{Z}\right)$ is a (possibly extended) metric space and $I$ is an interval of $\mathbb{R}$, a curve $\mathrm{z}: I \rightarrow Z$ is absolutely continuous if there exists $m \in \mathrm{L}^{1}(I)$ such that

$$
\mathrm{d}_{Z}\left(\mathrm{z}\left(t_{0}\right), \mathrm{z}\left(t_{1}\right)\right) \leq \int_{t_{0}}^{t_{1}} m(t) \mathrm{d} t \quad \text { whenever } t_{0}, t_{1} \in I, t_{0}<t_{1} .
$$

Its metric derivative $\left|z^{\prime}\right|_{d_{Z}}$ (we will omit the index $d_{Z}$ when the choice of the metric is clear from the context) is the Borel function defined by

$$
\left|\mathrm{z}^{\prime}\right|_{\mathrm{d}_{Z}}(t):=\limsup _{h \rightarrow 0} \frac{\mathrm{d}_{Z}(\mathrm{z}(t+h), \mathrm{z}(t))}{|h|}
$$

and it is possible to show (see [2]) that the lim sup above is in fact a limit for $\mathscr{L}^{1}$-a.e. points in $I$ and it provides the minimal (up to possible modifications in $\mathscr{L}^{1}$-negligible sets) function $m$ for which (8.1) holds. We will denote by $\operatorname{AC}^{p}(I ; Z)$ the class of all absolutely continuous curves $\mathrm{z}: I \rightarrow Z$ with $\left|\mathrm{z}^{\prime}\right| \in \mathrm{L}^{p}(I)$; when $I$ is an open set of $\mathbb{R}$, we will also consider the local space $\operatorname{AC}_{l o c}^{p}(I ; Z)$. If $Z$ is complete and separable then $\mathrm{AC}^{p}([0,1] ; Z)$ is a Borel set in the space $\mathrm{C}([0,1] ; Z)$ endowed with the topology of uniform convergence. (This property can be extended to the framework of extended metric-topological spaces, see [3].)

A curve $\mathrm{z}:[0,1] \rightarrow Z$ is a (minimal, constant speed) geodesic if

$$
\mathrm{d}_{Z}\left(\mathrm{z}\left(t_{0}\right), \mathrm{z}\left(t_{1}\right)\right)=\left|t_{1}-t_{0}\right| \mathrm{d}_{Z}(\mathrm{z}(0), \mathrm{z}(1)) \text { for every } t_{0}, t_{1} \in[0,1] \text {. }
$$

In particular $\mathrm{z}$ is Lipschitz and $\left|\mathrm{z}^{\prime}\right| \equiv \mathrm{d}_{Z}\left(\mathrm{z}\left(t_{0}\right), \mathrm{z}\left(t_{1}\right)\right)$ in $[0,1]$. We denote by $\mathrm{Geo}(Z) \subset \mathrm{C}([0,1] ; Z)$ the closed subset of all the geodesics.

By using the fact that $\int_{0}^{1} f^{2} \mathrm{~d} t \geq\left(\int_{0}^{1} f \mathrm{~d} t\right)^{2}$ with equality if and only if $f$ is constant a.e. in $(0,1)$, it is easy to check that a curve

$\mathrm{z} \in \operatorname{AC}^{2}([0,1] ; Z)$ is a geodesic if and only if $\int_{0}^{1}\left|\mathrm{z}^{\prime}\right|_{\mathrm{d}_{Z}}^{2} \mathrm{~d} t \leq \mathrm{d}_{Z}^{2}(\mathrm{z}(0), \mathrm{z}(1))$;

notice that the opposite inequality in (8.4) is satisfied along any curve. 
A metric space $\left(Z, \mathrm{~d}_{Z}\right)$ is called a length (or intrinsic) space if the distance between arbitrary pairs of points can be obtained as the infimum of the length of the absolutely continuous curves connecting them; by a simple reparametrization technique (see e.g. [2, Lem. 1.1.4]), this property is equivalent to assume that for every pair of points $z_{0}, z_{1} \in Z$ at finite distance and every $\kappa>1$ there exists a Lipschitz curve $\mathrm{z}_{\kappa}:[0,1] \rightarrow Z$ such that

$$
z(i)=z_{i}, i=0,1, \quad\left|\mathrm{z}^{\prime}\right|_{\mathrm{d}_{Z}}(t) \leq \kappa \mathrm{d}_{Z}\left(z_{0}, z_{1}\right) \text { for every } t \in[0,1] .
$$

$\left(Z, \mathrm{~d}_{Z}\right)$ is called a geodesic (or strictly intrinsic) space if every pair of points $z_{0}, z_{1}$ at finite distance can be joined by a geodesic (for which (8.5) holds with $\kappa=1)$.

Geodesics in $\mathfrak{C}$. If $(X, d)$ is a geodesic (resp. length) space, then also $\mathfrak{C}$ is a geodesic (resp. length) space, cf. [10, Sect.3.6]. The geodesic connecting a point $\mathfrak{y}=[x, r]$ with $\mathfrak{o}$ is

$$
\mathfrak{y}(t)=[x, t r]=\mathfrak{y} \cdot t \text { for } t \in[0,1] .
$$

If $x_{1}, x_{2} \in X$ with $\mathrm{d}\left(x_{1}, x_{2}\right) \geq \pi$, then a geodesic between $\mathfrak{y}_{i}=\left[x_{i}, r_{i}\right]$ can be easily obtained by joining two geodesics connecting $\mathfrak{y}_{i}$ to $\mathfrak{o}$ as before; observe that in this case $\mathrm{d}_{\mathfrak{C}}\left(\mathfrak{y}_{1}, \mathfrak{y}_{2}\right)=r_{1}+r_{2}$.

In the case when $\mathrm{d}\left(x_{1}, x_{2}\right)<\pi$ and $r_{1}, r_{2}>0$, every geodesic $\mathfrak{y}: I \rightarrow \mathfrak{C}$ connecting $\mathfrak{y}_{1}$ to $\mathfrak{y}_{2}$ is associated to a geodesic $\mathrm{x}$ in $X$ joining $x_{1}$ to $x_{2}$ and parametrized with unit speed in the interval $\left[0, \mathrm{~d}\left(x_{1}, x_{2}\right)\right]$. To find the radius $r(t)$, we use the complex plane $\mathbb{C}$ and write the curve connecting $z_{1}=r_{1} \in \mathbb{C}$ to $z_{2}=r_{2} \exp \left(\mathrm{id}\left(x_{1}, x_{2}\right)\right) \in \mathbb{C}$ in polar coordinates, namely

$$
\begin{aligned}
\mathrm{z}(t) & =r(t) \exp (\mathrm{i} \theta(t)), \\
r^{2}(t) & =(1-t)^{2} r_{1}^{2}+t^{2} r_{2}^{2}+2 t(1-t) r_{1} r_{2} \cos \left(\mathrm{d}\left(x_{1}, x_{2}\right)\right), \\
\cos (\theta(t)) & =\frac{(1-t) r_{1}+t r_{2} \cos \left(\mathrm{d}\left(x_{1}, x_{2}\right)\right)}{r(t)}, \theta(t) \in[0, \pi],
\end{aligned}
$$

and then the geodesic curve and the distance in $\mathfrak{C}$ take the form

$$
\mathfrak{y}(t)=[\mathrm{x}(\theta(t)), r(t)], \quad \mathbf{d}_{\mathfrak{C}}\left(\mathfrak{y}_{1}, \mathfrak{y}_{2}\right)=\left|z_{2}-z_{1}\right| .
$$

Absolutely continuous curves in $\mathfrak{C}$. We want to obtain now a simple characterizations of absolutely continuous curves in $\mathfrak{C}$. If $t \mapsto \mathfrak{y}(t)$ is a continuous curve in $\mathfrak{C}$, with $t \in[0,1]$, is clear that $\mathrm{r}(t):=\mathrm{r}(\mathfrak{y}(t))$ is a continuous curve with values in $[0, \infty)$. We can then consider the open set $O_{\mathrm{r}}=\mathrm{r}^{-1}((0, \infty))$ and the map $\mathrm{x}:[0,1] \rightarrow X$ defined by $\mathrm{x}(t):=\mathrm{x}(\mathfrak{y}(t))$, whose restriction to $O_{\mathrm{r}}$ is also continuous. Thus any continuous curve $\mathfrak{y}: I \rightarrow \mathfrak{C}$ can be lifted to a pair of maps $\mathrm{y}=\mathrm{y} \circ \mathfrak{y}=(\mathrm{x}, \mathrm{r}):[0,1] \rightarrow Y$ with $\mathrm{r}$ continuous and $\mathrm{x}$ 
continuous on $O_{\mathrm{r}}$ and constant on its complement. Conversely, it is clear that starting from a pair $\mathrm{y}=(\mathrm{x}, \mathrm{r})$ as above, then $\mathfrak{y}=\mathfrak{p} \circ \mathrm{y}$ is continuous in $\mathfrak{C}$. We thus introduce the set

$$
\begin{aligned}
\widetilde{\mathrm{C}}([0,1] ; Y):= & \{\mathrm{y}=(\mathrm{x}, \mathrm{r}):[0,1] \rightarrow Y: \\
& \left.\mathrm{r} \in \mathrm{C}\left([0,1] ; \mathbb{R}_{+}\right), \mathrm{x}_{\left.\right|_{O_{\mathrm{r}}}} \text { is continuous }\right\}
\end{aligned}
$$

and for $p \geq 1$ the analogous spaces

$$
\begin{aligned}
\widetilde{\operatorname{AC}^{p}}([0,1] ; Y):=\{\mathrm{y} & =(\mathrm{x}, \mathrm{r}): \mathrm{r} \in \mathrm{AC}^{p}\left([0,1] ; \mathbb{R}_{+}\right), \\
& \left.\mathrm{x}_{\left.\right|_{O_{\mathrm{r}}}} \in \operatorname{AC}_{l o c}^{p}\left(O_{\mathrm{r}} ; X\right), \mathrm{r}\left|\mathrm{x}^{\prime}\right| \in \mathrm{L}^{p}\left(O_{\mathrm{r}}\right)\right\} .
\end{aligned}
$$

If $\mathrm{y}=(\mathrm{x}, \mathrm{r}) \in \widetilde{\mathrm{AC}}^{p}([0,1] ; Y)$ we define the Borel map $\left|\mathrm{y}^{\prime}\right|:[0,1] \rightarrow \mathbb{R}_{+}$by

$$
\left|\mathrm{y}^{\prime}\right|^{2}(t):=\left|\mathrm{r}^{\prime}(t)\right|^{2}+\mathrm{r}^{2}(t)\left|\mathrm{x}^{\prime}\right|_{\mathrm{d}}^{2}(t) \quad \text { if } t \in O_{\mathrm{r}}, \quad\left|\mathrm{y}^{\prime}\right|(t)=0 \text { otherwise. }
$$

For absolutely continuous curves the following characterization holds:

Lemma 8.1 Let $\mathfrak{y} \in \mathrm{C}([0,1] ; \mathfrak{C})$ be lifted to $\mathrm{y}=\mathrm{y} \circ \mathfrak{y} \in \widetilde{\mathrm{C}}([0,1] ; Y)$. Then $\mathfrak{y} \in \operatorname{AC}^{p}(I ; \mathfrak{C})$ if and only if $\mathrm{y}=(\mathrm{x}, \mathrm{r}) \in \widetilde{\mathrm{AC}}^{p}([0,1] ; Y)$ and

$$
\left|\mathfrak{y}^{\prime}\right|_{\mathrm{d}_{\mathfrak{C}}}(t)=\left|\mathrm{y}^{\prime}\right|(t) \text { for } \mathscr{L}^{1} \text {-a.e. } t \in[0,1] .
$$

Proof By (7.5) one immediately sees that if $\mathfrak{y}=\mathfrak{p} \circ \mathrm{y} \in \mathrm{AC}^{p}([0,1] ; \mathfrak{C})$ then $\mathrm{r}$ belongs to $\mathrm{AC}^{p}([0,1] ; \mathbb{R})$ and $\mathrm{x} \in \operatorname{AC}_{\mathrm{loc}}^{p}\left(O_{\mathrm{r}} ; X\right)$. Since $\mathfrak{y}$ is absolutely continuous, we can evaluate the metric derivative at a.e. $t \in O_{\mathrm{r}}$ where also $\mathrm{r}^{\prime}$ and $\left|\mathrm{x}^{\prime}\right|$ exist: starting from (7.4) leads to the limit

$$
\begin{aligned}
\lim _{h \downarrow 0} & \frac{\mathrm{d}_{\mathfrak{C}^{(}}^{2}(\mathfrak{y}(t+h), \mathfrak{y}(t))}{h^{2}} \\
\quad & =\lim _{h \downarrow 0} \frac{|\mathrm{r}(t+h)-\mathrm{r}(t)|^{2}+4 \mathrm{r}(t+h) \mathrm{r}(t) \sin ^{2}\left(\frac{1}{2} \mathrm{~d}_{\pi}(\mathrm{x}(t+h), \mathrm{x}(t))\right)}{h^{2}} \\
\quad & =\left|\mathrm{r}^{\prime}(t)\right|^{2}+\mathrm{r}^{2}(t)\left|\mathrm{x}^{\prime}\right|_{\mathrm{d}}^{2}(t)
\end{aligned}
$$

which provides (8.12).

Moreover, the same calculations show that if the lifting y belongs to $\widetilde{\mathrm{AC}}^{p}([0,1] ; Y)$ then the restriction of $\mathfrak{y}$ to each connected component of $O_{\mathrm{r}}$ is absolutely continuous with metric velocity given by $(8.12)$ in $L^{p}(0,1)$. Since $\mathfrak{y}$ is globally continuous and constant in $[0,1] \backslash O_{\mathrm{r}}$, we conclude that $\mathfrak{y} \in \mathrm{AC}^{p}([0,1] ; \mathfrak{C})$. 
As a consequence, in a length space, we get the variational representation formula

$$
\begin{aligned}
\mathrm{d}_{\mathfrak{C}^{2}}^{2}\left(\mathfrak{y}_{0}, \mathfrak{y}_{1}\right)=\inf & \left\{\int_{[0,1] \cap\{\mathrm{r}>0\}}\left(\mathrm{r}^{2}(t)\left|\mathrm{x}^{\prime}\right|_{\mathrm{d}}^{2}(t)+\left|\mathrm{r}^{\prime}(t)\right|^{2}\right) \mathrm{d} t:\right. \\
& \left.(\mathrm{x}, \mathrm{r}) \in \widetilde{\mathrm{AC}}^{2}([0,1] ; Y),[\mathrm{x}(i), \mathrm{r}(i)]=\mathfrak{y}_{i}, i=0,1\right\} .
\end{aligned}
$$

Remark 8.2 (The Euclidean case) In the Euclidean case $X=\mathbb{R}^{d}$ with the usual Euclidean distance $\mathrm{d}\left(x_{1}, x_{2}\right):=\left|x_{1}-x_{2}\right|$ we can give a more explicit interpretation of the metric velocity (8.12) and write a simple duality formula for the chain rule of smooth functions that will turn out to be useful in Sect. 8.5.

For $\mathfrak{y}=[\mathrm{x}, \mathrm{r}] \in \mathrm{AC}^{2}([0,1] ; \mathfrak{C})$, we can define a Borel vector field $\mathfrak{y}_{\mathfrak{C}}^{\prime}$ : $[0,1] \rightarrow \mathbb{R}^{d+1}$ by

$$
\mathfrak{y}_{\mathfrak{C}}^{\prime}(t):= \begin{cases}\left(\mathrm{r}(t) \mathrm{x}^{\prime}(t), \mathrm{r}^{\prime}(t)\right) & \text { whenever } \mathrm{r}(t) \neq 0 \text { and the derivatives exist, } \\ (0,0) & \text { otherwise. }\end{cases}
$$

Then, (8.12) yields $\left|\mathfrak{y}^{\prime}\right|_{\mathfrak{d}_{\mathfrak{C}}}(t)=\left|\mathfrak{y}_{\mathfrak{C}^{\mathfrak{c}}}^{\prime}(t)\right|_{\mathbb{R}^{d+1}}$ for $\mathscr{L}^{1}$-a.e. $t \in(0,1)$ and the Euclidean norm of $\mathfrak{y}_{\mathfrak{C}}^{\prime}(t)$ corresponds to the Riemannian norm of $\mathfrak{y}^{\prime}$ with respect to the metric tensor $g_{[x, r]}(\dot{x}, \dot{r}):=r^{2}|\dot{x}|^{2}+\dot{r}^{2}$.

For $\xi \in C^{1}\left(\mathbb{R}^{d} \times[0,1]\right)$ we set $\zeta([x, r], t):=\xi(x, t) r^{2}$ and obtain $\partial_{t} \zeta([x, r], t):=\partial_{t} \xi(x, t) r^{2}$. Now defining the Borel map $\mathrm{D}_{\mathfrak{C} \zeta}: \mathfrak{C} \rightarrow$ $\left(\mathbb{R}^{d+1}\right)^{*}$ via

$$
\mathrm{D}_{\mathfrak{C}} \zeta(\mathfrak{y}, t):= \begin{cases}\left(r \mathrm{D}_{x} \xi(x, t), 2 r \xi(x, t)\right) & \text { for } \mathfrak{y} \neq \mathfrak{o} \\ (0,0) & \text { otherwise }\end{cases}
$$

we see that the map $t \mapsto \zeta(\mathfrak{y}(t), t)$ is absolutely continuous and satisfies

$$
\frac{\mathrm{d}}{\mathrm{d} t} \zeta(\mathfrak{y}(t), t)=\partial_{t} \zeta(\mathfrak{y}(t), t)+\left\langle\mathrm{D}_{\mathfrak{C}} \zeta(\mathfrak{y}(t), t), \mathfrak{y}_{\mathfrak{C}^{\prime}}^{\prime}(t)\right\rangle_{\mathbb{R}^{d+1}}
$$

$\mathscr{L}^{1}$-a.e. in $(0,1)$.

Note that the first component of $\mathrm{D}_{\mathfrak{C}} \zeta$ contains the factor $r$ rather than $r^{2}$, since $\mathfrak{y}_{\mathfrak{C}}^{\prime}$ in (8.12) already has one factor $r$ in its first component. The Euclidean norm of $\mathrm{D}_{\mathfrak{C}} \zeta$ corresponds to the dual Riemannian norm of the differential of $\zeta$.

\subsection{Lifting of absolutely continuous curves and geodesics}

Dynamic plans and time-dependent marginals. Let $\left(Z, \mathrm{~d}_{Z}\right)$ be a complete and separable metric space. A dynamic plan $\pi$ in $Z$ is a probability measure 
in $\mathcal{P}(\mathrm{C}(I ; Z))$, and we say that $\pi$ has finite 2-energy if it is concentrated on $\operatorname{AC}^{2}(I ; Z)$ and

$$
\int\left(\int_{0}^{1}\left|\mathrm{z}^{\prime}\right|_{\mathrm{d}_{Z}}^{2}(t) \mathrm{d} t\right) \mathrm{d} \boldsymbol{\pi}(\mathrm{z})<\infty .
$$

We denote by $\mathrm{e}_{t}$ the evaluation map on $\mathrm{C}(I ; Z)$ given by $\mathrm{e}_{t}(\mathrm{z}):=\mathrm{z}(t)$. If $\pi$ is a dynamic plan, $\alpha_{t}=\left(\mathrm{e}_{t}\right)_{\sharp} \boldsymbol{\pi} \in \mathcal{M}(Z)$ is its marginal at time $t \in I$ and the curve $t \mapsto \alpha_{t}$ belongs to $\mathrm{C}\left(I ;\left(\mathcal{M}(Z), \mathrm{W}_{\mathrm{d}_{Z}}\right)\right)$. If moreover $\pi$ is a dynamic plan with finite 2-energy, then $\alpha \in \mathrm{AC}^{2}\left(I ;\left(\mathcal{M}(Z), \mathrm{W}_{\mathrm{d}_{Z}}\right)\right.$ ) (see [33, Thm.4]).

We say that $\pi$ is an optimal geodesic plan between $\alpha_{0}, \alpha_{1} \in \mathcal{P}(Z)$ if $\left(\mathrm{e}_{i}\right)_{\sharp} \boldsymbol{\pi}=\alpha_{i}$ for $i=0,1$, if it is a dynamic plan concentrated on $\operatorname{Geo}(Z)$, and if

$$
\int \mathrm{d}_{Z}^{2}(\mathrm{z}(0), \mathrm{z}(1)) \mathrm{d} \boldsymbol{\pi}(\mathrm{z})=\iint_{0}^{1}\left|\mathrm{z}^{\prime}\right|^{2} \mathrm{~d} t \mathrm{~d} \boldsymbol{\pi}(\mathrm{z})=\mathrm{W}_{\mathrm{d}_{\mathrm{Z}}}^{2}\left(\alpha_{0}, \alpha_{1}\right) .
$$

Recalling (8.4), one immediately sees that for every dynamic plan $\pi$ concentrated on $\mathrm{AC}^{2}([0,1] ; Z)$ with $\left(\mathrm{e}_{i}\right)_{\sharp} \pi=\alpha_{i}$ the condition

$$
\iint_{0}^{1}\left|\mathrm{z}^{\prime}\right|^{2} \mathrm{~d} t \mathrm{~d} \pi(\mathrm{z}) \leq \mathrm{W}_{\mathrm{d}_{\mathrm{Z}}}^{2}\left(\alpha_{0}, \alpha_{1}\right)
$$

is sufficient to conclude that $\pi$ is an optimal geodesic plan for $\alpha_{0}, \alpha_{1} \in \mathcal{P}(Z)$.

When $Z=\mathfrak{C}$ we will denote by $\mathfrak{h}_{t}^{2}=\mathfrak{h}^{2} \circ\left(\mathrm{e}_{t}\right)_{\sharp}$ the homogeneous marginal at time $t \in I$. Since $\mathfrak{h}^{2}: \mathcal{P}(\mathfrak{C}) \rightarrow \mathcal{N}(X)$ is 1-Lipschitz (cf. Corollary 7.13), it follows that for every dynamic plan $\pi$ with finite 2-energy the curve $\mu_{t}:=$ $\mathfrak{h}^{2} \alpha_{t}=\mathfrak{h}_{t}^{2} \pi$ belongs to $\mathrm{AC}^{2}(I ;(\mathcal{M}(X), \mathrm{HK}))$ and moreover

$$
\left|\mu_{t}^{\prime}\right|_{\mathrm{HK}}^{2} \leq \int\left|\mathfrak{y}^{\prime}\right|_{\mathrm{d}_{\mathfrak{C}}}^{2}(t) \mathrm{d} \boldsymbol{\pi}(\mathfrak{y}) \text { for a.e. } t \in(0,1)
$$

A simple consequence of this property is that $(\mathcal{M}(X), \mathrm{HK})$ inherits the length (or geodesic) property of $(X, d)$.

Proposition $8.3(\mathcal{M}(X), \mathrm{KK})$ is a length (resp. geodesic) space if and only if $(X, \mathrm{~d})$ is a length (resp. geodesic) space.

Proof Let us first suppose that $(X, \mathrm{~d})$ is a length space (the argument in the geodesic case is completely equivalent) and let $\mu_{i} \in \mathcal{M}(X)$. By Corollary 7.7 we find $\alpha_{i} \in \mathcal{P}_{2}(\mathfrak{C})$ such that $\mathfrak{h}^{2} \alpha_{i}=\mu_{i}$ and $\mathrm{H}\left(\mu_{1}, \mu_{2}\right)=\mathbf{W}_{\mathfrak{d}_{\mathfrak{C}}}\left(\alpha_{1}, \alpha_{2}\right)$. Since $\mathfrak{C}$ is a length space, it is well known that $\mathcal{P}_{2}(\mathfrak{C})$ is a length space (see [47]); recalling (8.5), for every $\kappa>1$ there exists $\alpha \in \operatorname{Lip}\left([0,1] ;\left(\mathcal{P}_{2}(\mathfrak{C}), W_{d_{\mathfrak{c}}}\right)\right.$ ) connecting $\alpha_{1}$ to $\alpha_{2}$ such that $\left|\alpha^{\prime}\right| \mathrm{W}_{\mathrm{d}_{\mathfrak{C}}} \leq \kappa \mathrm{W}_{\mathrm{d}_{\mathfrak{C}}}\left(\alpha_{1}, \alpha_{2}\right)$. Setting $\mu_{t}:=\mathfrak{h}^{2} \alpha_{t}$ we obtain a Lipschitz curve connecting $\mu_{1}$ to $\mu_{2}$ with length $\leq \kappa \mathrm{H}\left(\mu_{1}, \mu_{2}\right)$. 
The converse property is a consequence of the next representation Theorem 8.4 and the fact that if $\left(\mathcal{P}_{2}(\mathfrak{C}), W_{d}\right)$ is a length (resp. geodesic) space, then $\mathfrak{C}$ and thus $X$ are length (resp. geodesic) spaces.

We want to prove the converse representation result that every absolutely continuous curve $\mu:[0,1] \rightarrow(\mathcal{M}(X), \mathrm{HK})$ can be written via a dynamic plan $\pi$ as $\mu_{t}=\mathfrak{h}_{t}^{2} \pi$. The argument only depends on the metric properties of the Lipschitz submersion $\mathfrak{h}$.

Theorem 8.4 Let $\left(\mu_{t}\right)_{t \in[0,1]}$ be a curve in $\mathrm{AC}^{p}([0,1] ;(\mathcal{M}(X), \mathrm{H})), p \in$ $[1, \infty]$, with

$$
\Theta:=\sqrt{\mu_{0}(X)}+\int_{0}^{1}\left|\mu^{\prime}\right|_{\mathrm{H}} \mathrm{d} t .
$$

Then there exists a curve $\left(\alpha_{t}\right)_{t \in[0,1]}$ in $\mathrm{AC}^{p}\left([0,1] ;\left(\mathcal{P}_{2}(\mathfrak{C}), \mathrm{W}_{\mathrm{d}_{\mathfrak{C}}}\right)\right)$ such that $\alpha_{t}$ is concentrated on $\mathfrak{C}[\Theta]$ for every $t \in[0,1]$ and

$$
\mu_{t}=\mathfrak{h}^{2} \alpha_{t} \text { in }[0,1], \quad\left|\mu_{t}^{\prime}\right|_{\mathfrak{K}}=\left|\alpha_{t}^{\prime}\right| \mathrm{W}_{\mathrm{d}_{\mathfrak{C}}} \text { for a.e. } t \in(0,1) \text {. }
$$

Moreover, when $p=2$, there exists a dynamic plan $\pi \in \mathcal{P}\left(\mathrm{AC}^{2}([0,1] ; \mathfrak{C})\right)$ such that

$$
\begin{gathered}
\alpha_{t}=\left(\mathrm{e}_{t}\right)_{\sharp} \boldsymbol{\pi}, \quad \mu_{t}=\mathfrak{h}_{t}^{2} \boldsymbol{\pi}=\mathfrak{h}^{2} \alpha_{t} \text { in }[0,1], \\
\left|\mu_{t}^{\prime}\right|_{H_{\mathfrak{K}}}^{2}=\left|\alpha_{t}^{\prime}\right|_{\mathrm{W}_{\mathrm{d}_{\mathfrak{C}}}}^{2}=\int\left|\mathfrak{y}^{\prime}\right|_{\mathrm{d}_{\mathfrak{C}}}^{2}(t) \mathrm{d} \boldsymbol{\pi}(\mathfrak{y}) \text { for a.e. } t \in(0,1) .
\end{gathered}
$$

Proof By Lisini's lifting Theorem [33, Theorem 5] (8.23) is a consequence of the first part of the statement and (8.22) in the case $p=2$. It is therefore sufficient to prove that for a given $\mu \in \mathrm{AC}([0,1] ;(\mathcal{M}(X), \mathrm{HK}))$ there exists a curve $\alpha \in \operatorname{AC}\left([0,1] ;\left(\mathcal{P}_{2}(\mathfrak{C}), \mathbf{W}_{\mathfrak{d}_{\mathfrak{C}}}\right)\right)$ such that $\mu_{t}=\mathfrak{h}^{2}\left(\alpha_{t}\right)$ and $\left|\mu_{t}^{\prime}\right|=\left|\alpha_{t}^{\prime}\right|$ a.e. in $(0,1)$. By a standard reparametrization technique (see e.g. [2, Lem. 1.1.4]), we may assume that $\mu$ is Lipschitz continuous and $\left|\mu_{t}^{\prime}\right|=L$.

We divide the interval $I=[0,1]$ into $2^{N}$-intervals of size $2^{-N}$, namely $I_{i}^{N}:=\left[t_{i-1}^{N}, t_{i}^{N}\right]$ with $t_{i}^{N}:=i 2^{-N}$ for $i=1, \ldots, 2^{N}$. Setting $\mu_{i}^{N}:=\mu_{t_{i}^{N}}$ we can apply the Gluing Lemma 7.11 (starting from $i=0$ to $2^{N}$ ) to obtain measures $\alpha_{i}^{N} \in \mathcal{P}_{2}(\mathfrak{C})$ such that

$$
\mathfrak{h}\left(\alpha_{i}^{N}\right)=\mu_{i}^{N}, \quad W_{\mathrm{d}_{\mathfrak{C}}}\left(\alpha_{i}^{N}, \alpha_{i+1}^{N}\right)=\mathrm{H}\left(\mu_{i}^{N}, \mu_{i+1}^{N}\right) \leq L 2^{-N},
$$

and concentrated on $\mathfrak{C}\left[\Theta_{N}\right]$ where

$$
\Theta_{N}=\sqrt{\mu_{0}(X)}+\sum_{i=1}^{2^{N}} \mathrm{H}\left(\mu_{i-1}^{N}, \mu_{i}^{N}\right) \leq \Theta .
$$


Thus if $t$ is a dyadic point, we obtain a sequence of probability measures $\alpha^{N}(t) \in \mathcal{P}_{2}(\mathfrak{C})$ concentrated on $\mathfrak{C}[\Theta]$ with $\mathfrak{h}^{2}\left(\alpha^{N}(t)\right)=\mu_{t}$ and such that $W_{\mathrm{d}_{\mathfrak{C}}}\left(\alpha^{N}(t), \alpha^{N}(s)\right) \leq L|t-s|$ if $s=m 2^{-N}$ and $t=n 2^{-N}$ are dyadic points in the same grid. By the compactness Lemma 7.3 and a standard diagonal argument, we can extract a subsequence $N(k)$ such that $\alpha_{N(k)}(t)$ converges to $\alpha(t)$ in $\left(\mathcal{P}_{2}(\mathfrak{C}), W_{\mathrm{d}_{\mathfrak{C}}}\right)$ for every dyadic point $t$. Since $W_{\mathrm{d}_{\mathfrak{C}}}(\alpha(s), \alpha(t)) \leq$ $L|t-s|$ for every dyadic $s, t$, we can extend $\alpha$ to a $L$-Lipschitz curve, still denoted by $\alpha$, which satisfies $\mathfrak{h}^{2}(\alpha(t))=\mu_{t}$. Since $\mathfrak{h}^{2}$ is 1 -Lipschitz, we conclude that $\left|\alpha^{\prime}\right|(t)=\left|\mu_{t}^{\prime}\right|$ a.e. in $(0,1)$.

Corollary 8.5 Let $\left(\mu_{t}\right)_{t \in[0,1]}$ be a curve in $\mathrm{AC}^{2}([0,1] ;(\mathcal{M}(X), \mathrm{H}))$ and let $\Theta$ as in (8.21). Then there exists a dynamic plan $\tilde{\pi}$ in $\mathcal{P}(\widetilde{C}([0,1] ; Y))$ concentrated on $\widetilde{\mathrm{AC}}^{2}([0,1] ; Y)$ such that $\alpha_{t}=\left(\mathrm{e}_{t}\right)_{\sharp} \boldsymbol{\pi}$ is concentrated in $X \times[0, \Theta]$, that $\mu_{t}=\mathrm{h}^{2}\left(\left(\mathrm{e}_{t}\right)_{\sharp} \pi\right)$, and that

$$
\left|\mu_{t}^{\prime}\right|_{\mathrm{HK}}^{2}=\int\left|\mathrm{y}^{\prime}\right|^{2}(t) \mathrm{d} \boldsymbol{\pi}(\mathrm{y}) \text { for } \mathscr{L}^{1} \text {-a.e. } t \in[0,1],
$$

where $\left|\mathrm{y}^{\prime}\right|$ is defined in (8.11).

Another important consequence of the previous representation result is a precise characterization of the geodesics in $(\mathcal{M}(X), \mathrm{H})$.

Theorem 8.6 (Geodesics in $(\mathcal{M}(X), \mathrm{K}))$

(i) If $\left(\mu_{t}\right)_{t \in[0,1]}$ is a geodesic in $(\mathcal{M}(X), \mathrm{K})$ then there exists an optimal geodesic plan $\pi$ in $\mathcal{P}(\mathrm{Geo}(\mathfrak{C}))$ (recall (8.18)) such that

(a) $\pi$-a.e. curve $\mathfrak{y}$ is a geodesic in $\mathfrak{C}$,

(b) $[0,1] \ni t \mapsto \alpha_{t}:=\left(\mathrm{e}_{t}\right)_{\sharp} \pi$ is a geodesic in $\left(\mathcal{P}_{2}(\mathfrak{C}), \mathrm{W}_{\mathrm{d}_{\mathfrak{C}}}\right)$, where all $\alpha_{t}$ are concentrated on $\mathfrak{C}[\Theta]$ with $\Theta^{2}=2\left(\mu_{0}(X)+\mathrm{H}^{2}\left(\mu_{0}, \mu_{1}\right)\right)$,

(c) $\mu_{t}=\mathfrak{h}_{t}^{2} \pi=\mathfrak{h}^{2} \alpha_{t}$ for every $t \in[0,1]$, and

(d) $\left(\mathrm{e}_{s}, \mathrm{e}_{t}\right)_{\sharp} \pi \in \mathrm{Opt}_{H_{K}}\left(\mu_{s}, \mu_{t}\right)$ if $0 \leq s<t \leq 1$.

(ii) If $(X, \mathrm{~d})$ is a geodesic space, for every $\mu_{0}, \mu_{1} \in \mathcal{M}(X)$ and every $\boldsymbol{\alpha} \in$ $\operatorname{Opt}_{\mathbb{K}}\left(\mu_{0}, \mu_{1}\right)$ there exists an optimal geodesic plan $\pi \in \mathcal{P}(\operatorname{Geo}(\mathfrak{C}))$ such that $\left(\mathrm{e}_{0}, \mathrm{e}_{1}\right)_{\sharp} \boldsymbol{\pi}=\boldsymbol{\alpha}$.

Proof The statement (i) is an immediate consequence of Theorem 8.4. Notice that $\left|\mu_{t}^{\prime}\right|_{\mathrm{HK}_{\mathrm{K}}}^{2}=\mathrm{HK}^{2}\left(\mu_{0}, \mu_{1}\right)$ in $(0,1)$ since $\left(\mu_{t}\right)_{t \in[0,1]}$ is a geodesic, so that (8.23) yields

$$
\iint_{0}^{1}\left|\mathfrak{y}^{\prime}\right|_{\mathrm{d}_{\mathfrak{C}}}^{2}(t) \mathrm{d} t \mathrm{~d} \boldsymbol{\pi}(\mathfrak{y})=\int_{0}^{1}\left|\mu_{t}^{\prime}\right|_{\mathfrak{H K}^{2}}^{2} \mathrm{~d} t=\mathrm{H}^{2}\left(\mu_{0}, \mu_{1}\right) \leq \mathrm{W}_{\mathfrak{d}_{\mathfrak{C}}}^{2}\left(\alpha_{0}, \alpha_{1}\right),
$$

so that $\boldsymbol{\pi}$ satisfies (8.19) in $\mathfrak{C}$ and we deduce that it is an optimal geodesic plan. 
Statement (ii) is a well known property [33, Thm.6] of the KantorovichWasserstein space $\left(\mathfrak{C}, W_{\mathfrak{d}_{\mathfrak{C}}}\right)$ in the case when $\mathfrak{C}$ is geodesic.

Theorem 8.4 also clarifies the relation between $\mathrm{HK}$ and $\mathrm{GK}$ introduced in Sect. 7.8.

Corollary 8.7 If $(X, \mathrm{~d})$ is separable and complete then $\mathrm{AC}^{2}([0,1]$; $(\mathcal{M}(X), G K))$ coincides with $\mathrm{AC}^{2}([0,1] ;(\mathcal{M}(X), \mathrm{H}))$ and for every curve $\mu \in \mathrm{AC}^{2}([0,1] ;(\mathcal{M}(X), \mathrm{GK}))$ we have

$$
\left|\mu^{\prime}\right|_{\mathrm{GK}}(t)=\left|\mu^{\prime}\right|_{\mathbb{H}}(t) \text { for } \mathscr{L}^{1} \text {-a.e. } t \in[0,1] .
$$

In particular if $(X, \mathrm{~d})$ is a length metric space then $\mathrm{H}$ is the length distance generated by $\mathrm{GK}$.

Proof Since $\mathrm{GK} \leq \mathrm{HK}$ it is clear that $\mathrm{AC}^{2}([0,1] ;(\mathcal{M}(X), \mathrm{HK})) \subset \mathrm{AC}^{2}([0,1]$; $(\mathcal{M}(X), \mathrm{GK}))$.

In order to prove the opposite inclusion and (8.26) it is sufficient to notice that the classes of absolutely continuous curves in $\mathfrak{C}$ w.r.t. $d_{\mathfrak{C}}$ and $g_{\mathfrak{C}}$ coincide with equal metric derivatives $\left|\mathfrak{y}^{\prime}\right|_{d_{\mathfrak{C}}}=\left|\mathfrak{y}^{\prime}\right|_{g_{\mathfrak{C}}}$. Since $G K=\mathrm{K}_{\mathrm{g}}$ is the Hellinger-Kantorovich distance induced by $\mathrm{g}$, the assertion follows by (8.23) of Theorem 8.4.

\subsection{Lower curvature bound in the sense of Alexandrov}

Let us first recall two possible definitions of Positively Curved (PC) spaces in the sense of Alexandrov, referring to [10] and to [11] for other equivalent definitions and for the more general case of spaces with curvature $\geq k, k \in$ $\mathbb{R}$. In the case of a smooth Riemannian manifold $(M, g)$ equipped with the Riemannian distance $d_{g}$ all the local definitions are equivalent to assume that the sectional curvature of $M$ is nonnegative (or bounded by $k \mathrm{~g}$, in the case of curvature $\geq k$ ).

According to Sturm [46], a metric space $\left(Z, d_{Z}\right)$ is a Positively Curved (PC) metric space in the large if for every choice of points $z_{0}, z_{1}, \ldots, z_{N} \in Z$ and coefficients $\lambda_{1}, \ldots, \lambda_{N} \in(0, \infty)$ we have

$$
\sum_{i, j=1}^{N} \lambda_{i} \lambda_{j} \mathrm{~d}_{Z}^{2}\left(z_{i}, z_{j}\right) \leq 2 \sum_{i, j=1}^{N} \lambda_{i} \lambda_{j} \mathrm{~d}_{Z}^{2}\left(z_{0}, z_{j}\right) .
$$

If every point of $Z$ has a neighborhood that is $\mathrm{PC}$, then we say that $Z$ is locally positively curved.

When the space $Z$ is geodesic, the above (local and global) definitions coincide with the corresponding one given by Alexandrov, which is based on 
triangle comparison: for every choice of $z_{0}, z_{1}, z_{2} \in Z$, every $t \in[0,1]$, and every point $z_{t}$ such that $\mathrm{d}_{Z}\left(z_{t}, z_{k}\right)=|k-t| \mathrm{d}_{Z}\left(z_{0}, z_{1}\right)$ for $k=0,1$ we have

$$
\mathrm{d}_{Z}^{2}\left(z_{2}, z_{t}\right) \geq(1-t) \mathrm{d}_{Z}^{2}\left(z_{2}, z_{0}\right)+t \mathrm{~d}_{Z}^{2}\left(z_{2}, z_{1}\right)-2 t(1-t) \mathrm{d}_{Z}^{2}\left(z_{0}, z_{1}\right) .
$$

When $Z$ is also complete, the local and the global definitions are equivalent [46, Corollary 1.5]. Next we provide conditions on $(X, \mathrm{~d})$ or $\left(\mathfrak{C}, \mathrm{d}_{\mathfrak{C}}\right)$ that guarantee that $(\mathcal{M}(X), \mathrm{HK})$ is a $\mathrm{PC}$ space.

Theorem 8.8 Let $(X, \mathrm{~d})$ be a metric space.

(i) If $X \subset \mathbb{R}$ is convex (i.e. an interval) endowed with the standard distance, then $(\mathcal{M}(X), \mathrm{H})$ is a PC space.

(ii) If $\left(\mathfrak{C}, \mathrm{d}_{\mathfrak{C}}\right)$ is a PC space in the large, $c f .(8.27)$, then $(\mathcal{M}(X), \mathrm{H}(X))$ is a PC space.

(iii) If $(X, \mathrm{~d})$ is separable, complete and geodesic, then $(\mathcal{M}(X), \mathrm{H})$ is a PC space if and only if $(X, \mathrm{~d})$ has locally curvature $\geq 1$.

Before we go into the proof of this result, we highlight that for a compact convex subset $\Omega \subset \mathbb{R}^{d}$ with $d \geq 2$ equipped with the Euclidean distance, the space $(\mathcal{M}(\Omega), \mathrm{H})$ is not PC, see [30, Sect.5.6] for an explicit construction showing the semiconcavity of the squared distance fails.

Proof Let us first prove statement (ii). If $\left(\mathfrak{C}, d_{\mathfrak{C}}\right)$ is a PC space then also $\left(\mathcal{P}_{2}(\mathfrak{C}), W_{\mathrm{d}_{\mathfrak{C}}}\right)$ is a PC space [47]. Applying Corollary 7.13, for every choice of $\mu_{i} \in \mathcal{M}(X), i=0, \ldots, N$, we can then find measures $\beta_{i} \in \mathcal{P}_{2}(\mathfrak{C})$ such that

$$
\mathrm{W}_{\mathrm{d}_{\mathfrak{C}}}\left(\beta_{0}, \beta_{i}\right)=\mathrm{H}\left(\mu_{0}, \mu_{i}\right) \text { for } i=1, \ldots, N,
$$

where it is crucial that $\beta_{0}$ is the same for every $i$. It then follows that

$$
\begin{aligned}
\sum_{i, j=1}^{N} \lambda_{i} \lambda_{j} \mathrm{HK}^{2}\left(\mu_{i}, \mu_{j}\right) & \leq \sum_{i, j=1}^{N} \lambda_{i} \lambda_{j} \mathrm{~W}_{\mathrm{d}_{\mathfrak{C}}}^{2}\left(\beta_{i}, \beta_{j}\right) \leq 2 \sum_{i, j=1}^{N} \lambda_{i} \lambda_{j} \mathrm{~W}_{\mathrm{d}_{\mathfrak{C}}}^{2}\left(\beta_{0}, \beta_{i}\right) \\
& =2 \sum_{i, j=1}^{N} \lambda_{i} \lambda_{j} \mathrm{H}^{2}\left(\mu_{0}, \mu_{i}\right) .
\end{aligned}
$$

Let us now consider (iii) " $\Rightarrow$ ": If $(\mathcal{M}(X), \mathrm{H})$ is $\mathrm{PC}$, we have to prove that $(X, \mathrm{~d})$ has locally curvature $\geq 1$. By Theorem [10, Thm. 4.7.1] it is sufficient to prove that $\mathfrak{C} \backslash\{\mathfrak{o}\}$ is locally PC to conclude that $(X, \mathrm{~d})$ has locally curvature $\geq 1$. We thus select points $\mathfrak{y}_{i}=\left[x_{i}, r_{i}\right], i=0,1,2$, in a sufficiently small neighborhood of $\mathfrak{y}=[x, r]$ with $r>0$, so that $\mathrm{d}\left(x_{i}, x_{j}\right)<\pi / 2$ for every $i, j$ and $r_{i}, r_{j}>0$. We also consider a geodesic $\mathfrak{y}_{t}=\left[x_{t}, s_{t}\right], t \in[0,1]$, connecting $\mathfrak{y}_{0}$ to $\mathfrak{y}_{1}$, thus satisfying $\mathrm{d}_{\mathfrak{C}}\left(\mathfrak{y}_{t}, \mathfrak{y}_{i}\right)=|i-t| \mathrm{d}\left(\mathfrak{y}_{0}, \mathfrak{y}_{1}\right)$ for $i=0,1$. 
Setting $\mu_{i}:=r_{i} \delta_{x_{i}}, \mu_{t}:=s_{t} \delta_{x_{t}}$, it is easy to check (cf. [30, Sect. 3.3.1]) that

$$
\begin{aligned}
& \mathrm{H}\left(\mu_{i}, \mu_{j}\right)=\mathrm{d}_{\mathfrak{C}}\left(\mathfrak{y}_{i}, \mathfrak{y}_{j}\right) \text { for } i, j \in\{0,1,2\}, \\
& \mathrm{K}\left(\mu_{t}, \mu_{k}\right)=|k-t| \mathrm{H}\left(\mu_{0}, \mu_{1}\right) \text { for } k \in\{0,1\} .
\end{aligned}
$$

We can thus apply (8.28) to $\mu_{0}, \mu_{1}, \mu_{2}, \mu_{t}$ and obtain the corresponding inequality for $\mathfrak{y}_{0}, \mathfrak{y}_{1}, \mathfrak{y}_{2}, \mathfrak{y}_{t}$.

(iii) " $\Leftarrow$ ": In order to prove the converse property we apply Remark 7.12. For $\mu_{0}, \mu_{1}, \mu_{2}$, and $\mu_{3}=\mu_{t} \in \mathcal{M}(X)$ with $t \in[0,1]$ and $\mathrm{K}\left(\mu_{3}, \mu_{k}\right)=$ $|k-t| \mathrm{H}\left(\mu_{0}, \mu_{1}\right)$, we find a plan $\alpha \in \mathcal{P}\left(X_{0} \times X_{1} \times X_{2} \times X_{3}\right)$ (with the usual convention to use copies of $X$ ) such that

$$
\mathfrak{h}_{i}^{2} \boldsymbol{\alpha}=\mu_{i}, \quad \int \mathrm{d}_{\mathfrak{C}}^{2}\left(\mathfrak{y}_{i}, \mathfrak{y}_{j}\right) \mathrm{d} \boldsymbol{\alpha}=\mathrm{HK}^{2}\left(\mu_{i}, \mu_{j}\right)
$$

for $(i, j) \in A=\{(0,3),(1,3),(2,3)\}$. The triangle inequality, the elementary inequality $t(1-t)(a+b)^{2} \leq(1-t) a^{2}+t b^{2}$, and the very definition of $\mathrm{HK}$ yield for $t \in(0,1)$ the estimate

$$
\begin{aligned}
t(1-t) \mathrm{HK}^{2}\left(\mu_{0}, \mu_{1}\right) \leq t(1-t) \int \mathrm{d}_{\mathfrak{C}}^{2}\left(\mathfrak{y}_{0}, \mathfrak{y}_{1}\right) \mathrm{d} \boldsymbol{\alpha} \\
\quad \leq \int t(1-t)\left(\left(\mathrm{d}_{\mathfrak{C}}\left(\mathfrak{y}_{0}, \mathfrak{y}_{3}\right)+\mathrm{d}_{\mathfrak{C}}\left(\mathfrak{y}_{3}, \mathfrak{y}_{1}\right)\right)^{2} \mathrm{~d} \boldsymbol{\alpha}\right. \\
\quad \leq \int(1-t) \mathrm{d}_{\mathfrak{C}}^{2}\left(\mathfrak{y}_{0}, \mathfrak{y}_{3}\right)+t \mathrm{~d}_{\mathfrak{C}}^{2}\left(\mathfrak{y}_{3}, \mathfrak{y}_{1}\right) \mathrm{d} \boldsymbol{\alpha} \\
\quad=(1-t) \mathrm{H}^{2}\left(\mu_{0}, \mu_{3}\right)+t \mathrm{H}^{2}\left(\mu_{3}, \mu_{1}\right)=t(1-t) \mathrm{HK}^{2}\left(\mu_{0}, \mu_{1}\right) .
\end{aligned}
$$

This series of inequalities shows in particular that

$$
\begin{aligned}
(1-t) \mathrm{d}_{\mathfrak{C}}^{2}\left(\mathfrak{y}_{0}, \mathfrak{y}_{3}\right)+t \mathrm{~d}_{\mathfrak{C}}^{2}\left(\mathfrak{y}_{3}, \mathfrak{y}_{1}\right) & =t(1-t)\left(\mathrm{d}_{\mathfrak{C}}\left(\mathfrak{y}_{0}, \mathfrak{y}_{3}\right)+\mathrm{d}_{\mathfrak{C}}\left(\mathfrak{y}_{3}, \mathfrak{y}_{1}\right)\right)^{2} \\
& =t(1-t) \mathrm{d}_{\mathfrak{C}}^{2}\left(\mathfrak{y}_{0}, \mathfrak{y}_{1}\right) \quad \alpha \text {-a.e. }
\end{aligned}
$$

so that

$$
\mathrm{d}_{\mathfrak{C}}\left(\mathfrak{y}_{0}, \mathfrak{y}_{3}\right)=t \mathrm{~d}_{\mathfrak{C}}\left(\mathfrak{y}_{0}, \mathfrak{y}_{1}\right) \text { and } \mathrm{d}_{\mathfrak{C}}\left(\mathfrak{y}_{3}, \mathfrak{y}_{1}\right)=(1-t) \mathrm{d}_{\mathfrak{C}}\left(\mathfrak{y}_{0}, \mathfrak{y}_{1}\right) \quad \boldsymbol{\alpha} \text {-a.e. }
$$

Moreover, $\pi_{\sharp}^{\mathfrak{y}_{0}, \mathfrak{y}_{1}} \boldsymbol{\alpha} \in \mathrm{Opt}_{\mathbb{H}_{\mathbb{K}}}\left(\mu_{0}, \mu_{1}\right)$, so that (8.31) holds for $(i, j) \in A^{\prime}=$ $A \cup\{(0,1)\}$.

By Theorem 7.20 we deduce that

$$
\mathrm{d}\left(\mathrm{x}_{i}, \mathrm{x}_{j}\right) \leq \pi / 2 \quad \boldsymbol{\alpha} \text {-a.e. for }(i, j) \in A^{\prime}
$$


If one of the points $\mathfrak{y}_{i}, i=0,1,2$, is the vertex $\mathfrak{o}$, then it is not difficult to check by a direct computation that

$$
\mathrm{d}_{\mathfrak{C}}^{2}\left(\mathfrak{y}_{2}, \mathfrak{y}_{3}\right) \geq(1-t) \mathrm{d}_{\mathfrak{C}}^{2}\left(\mathfrak{y}_{2}, \mathfrak{y}_{0}\right)+t \mathrm{~d}_{\mathfrak{C}^{2}}^{2}\left(\mathfrak{y}_{2}, \mathfrak{y}_{1}\right)-2 t(1-t) \mathrm{d}_{\mathfrak{C}}^{2}\left(\mathfrak{y}_{0}, \mathfrak{y}_{1}\right) .
$$

When $\mathfrak{y}_{i} \in \mathfrak{C} \backslash\{\mathfrak{o}\}$ for every $i=0,1,2$, we use $\mathrm{d}\left(\mathrm{x}_{0}, \mathrm{x}_{1}\right)+\mathrm{d}\left(\mathrm{x}_{1}, \mathrm{x}_{2}\right)+$ $\mathrm{d}\left(\mathrm{x}_{2}, \mathrm{x}_{0}\right) \leq \frac{3}{2} \pi<2 \pi$, and Theorem [10, Thm. 4.7.1] yields (8.32) because of the assumption that $X$ is PC. Integrating (8.32) w.r.t. $\boldsymbol{\alpha}$, by taking into account (8.31), the fact that $\left(\pi^{0}, \pi^{1}\right)_{\sharp} \boldsymbol{\alpha} \in \mathrm{Opt}_{\mathbb{K}_{K}}\left(\mu_{0}, \mu_{1}\right)$, and that

$$
\int \mathrm{d}_{\mathfrak{C}^{c}}^{2}\left(\mathfrak{y}_{2}, \mathfrak{y}_{i}\right) \mathrm{d} \boldsymbol{\alpha} \geq \mathrm{K}^{2}\left(\mu_{2}, \mu_{i}\right) \text { for } i=0,1,
$$

we obtain

$\mathrm{H}^{2}\left(\mu_{2}, \mu_{3}\right) \geq(1-t) \mathrm{H}^{2}\left(\mu_{2}, \mu_{0}\right)+t \mathrm{H}^{2}\left(\mu_{2}, \mu_{1}\right)-2 t(1-t) \mathrm{H}^{2}\left(\mu_{0}, \mu_{1}\right)$.

Finally, statement (i) is just a particular case of (iii).

As simple applications of the Theorem above we obtain that $\mathcal{N}(\mathbb{R})$ and $\mathcal{M}\left(\mathbb{S}^{d-1}\right)$ endowed with $\mathrm{K}$ are Positively Curved spaces.

\subsection{Duality and Hamilton-Jacobi equation}

In this section we will show the intimate connections of the duality formula of Theorem 7.21 with Lipschitz subsolutions of the Hamilton-Jacobi equation in $X \times(0,1)$ given by

$$
\partial_{t} \xi_{t}+\frac{1}{2}\left|\mathrm{D}_{X} \xi_{t}\right|^{2}+2 \xi_{t}^{2}=0
$$

and its counterpart in the cone space

$$
\partial_{t} \zeta_{t}+\frac{1}{2}\left|\mathbf{D}_{\mathfrak{C}} \zeta_{t}\right|^{2}=0
$$

Indeed, the first derivation of $\mathrm{HK}$ via LET was obtained by solving (8.33) for $X=\mathbb{R}^{d}$, see the remarks on the chronological development in Section A.

At a formal level, it is not difficult to check that solutions to (8.33) corresponds to the special class of solutions to (8.34) of the form

$$
\zeta_{t}([x, r]):=\xi_{t}(x) r^{2} .
$$

Indeed, still on the formal level we have the formula

$$
\left|\mathrm{D}_{\mathfrak{C}} \zeta\right|^{2}=\frac{1}{r^{2}}\left|\mathrm{D}_{X} \zeta\right|^{2}+\left|\partial_{r} \zeta\right|^{2}=\left|\mathrm{D}_{X} \xi\right|^{2} r^{2}+4 \xi^{2} r^{2} \text { if } \zeta=\xi r^{2}
$$


Since the Kantorovich-Wasserstein distance on $\mathcal{P}_{2}(\mathfrak{C})$ can be defined in duality with subsolutions to (8.34) via the Hopf-Lax formula (see e.g. $[3,50]$ ) and 2-homogeneous marginals are modeled on test functions as in (8.35), we can expect to obtain a dual representation for the Hellinger-Kantorovich distance on $\mathcal{M}(X)$ by studying the Hopf-Lax formula for initial data of the form $\zeta_{0}(x, r)=\xi_{0}(x) r^{2}$.

Slope and asymptotic Lipschitz constant. In order to give a metric interpretation to (8.33) and (8.34), let us first recall that for a locally Lipschitz function $f: Z \rightarrow \mathbb{R}$ defined in a metric space $\left(Z, \mathrm{~d}_{Z}\right)$ the metric slope $\left|\mathrm{D}_{Z} f\right|$ and the asymptotic Lipschitz constant $\left|\mathrm{D}_{Z} f\right|_{a}$ are defined by $[2,3,12]$

$$
\begin{aligned}
\left|\mathrm{D}_{Z} f\right|(z) & :=\limsup _{x \rightarrow z} \frac{|f(x)-f(z)|}{\mathrm{d}_{Z}(x, z)}, \\
\left|\mathrm{D}_{Z} f\right|_{a}(z) & :=\lim _{r \downarrow 0} \sup _{\substack{x, y \in B_{r}(z) \\
y \neq x}} \frac{|f(y)-f(x)|}{\mathrm{d}_{Z}(x, y)}
\end{aligned}
$$

with the convention that $\left|\mathrm{D}_{Z} f\right|(z)=\left|\mathrm{D}_{Z} f\right|_{a}(z)=0$ whenever $z$ is an isolated point. It is not difficult to check that $\left|\mathrm{D}_{Z} f\right|_{a}$ can also be defined as the minimal constant $L \geq 0$ such that there exists a function $G_{L}: Z \times Z \rightarrow[0, \infty)$ satisfying

$$
|f(x)-f(y)| \leq G_{L}(x, y) \mathrm{d}_{Z}(x, y), \quad \limsup _{x, y \rightarrow z} G_{L}(x, y) \leq L .
$$

Note that $\left|\mathrm{D}_{Z} f\right|_{a}$ is always an upper semicontinuous function clearly satisfying $\left|\mathrm{D}_{Z} f\right|_{a} \geq\left|\mathrm{D}_{Z} f\right|$. When $Z$ is a length space, (8.5) and the chain rule along Lipschitz curves easily yield

$$
|f(x)-f(y)| \leq \mathrm{d}_{Z}(x, y) \sup _{B_{r}(z)}\left|\mathrm{D}_{Z} f\right| \text { for every } x, y \in B_{2 r}(z),
$$

so that $\left|\mathrm{D}_{Z} f\right|_{a}$ is the upper semicontinuous envelope of the metric slope $\left|\mathrm{D}_{Z} f\right|$. We will often write $|\mathrm{D} f|,|\mathrm{D} f|_{a}$ whenever the space $Z$ will be clear from the context.

Remark 8.9 The notion of locally Lipschitz function and the value $\left|\mathrm{D}_{Z} f\right|_{a}$ does not change if we replace the distance $\mathrm{d}_{Z}$ with a distance $\tilde{\mathrm{d}}_{Z}$ of the form 


$$
\begin{aligned}
& \tilde{\mathrm{d}}_{Z}\left(z_{1}, z_{2}\right):=h\left(\mathrm{~d}_{Z}\left(z_{1}, z_{2}\right)\right) \text { for } z_{1}, z_{2} \in Z, \\
& \quad \text { with } h:[0, \infty) \rightarrow[0, \infty) \text { concave and } \lim _{r \downarrow 0} \frac{h(r)}{r}=1 .
\end{aligned}
$$

In particular, the truncated distances $\mathrm{d}_{Z} \wedge \kappa$ with $\kappa>0$, the distances $a \sin \left(\left(\mathrm{d}_{Z} \wedge \kappa\right) / a\right)$ with $a>0$ and $\kappa \in(0, a \pi / 2]$, and the distance $\mathrm{g}=g(\mathrm{~d})$ given by (7.74) yield the same asymptotic Lipschitz constant.

In the case of the cone space $\mathfrak{C}$ it is not difficult to see that the distance $d_{\mathfrak{C}}$ and $d_{\pi / 2, \mathfrak{C}}$ coincide in suitably small neighborhoods of every point $\mathfrak{y} \in \mathfrak{C} \backslash\{\mathfrak{o}\}$, so that they induce the same asymptotic Lipschitz constants in $\mathfrak{C} \backslash\{\mathfrak{o}\}$. The same property holds for $\mathfrak{g}_{\mathfrak{C}}$. In the case of the vertex $\mathfrak{o}$, relation (7.12) yields

$$
\left|\mathrm{D}_{\mathfrak{C}} f\right|_{a}(\mathfrak{o}) \leq\left|\mathrm{D}_{\left(\mathfrak{C}, \mathrm{d}_{\pi / 2, \mathfrak{C}}\right)} f\right|_{a}(\mathfrak{o}) \leq \sqrt{2}\left|\mathrm{D}_{\mathfrak{C}} f\right|_{a}(\mathfrak{o}) .
$$

The next result shows that the asymptotic Lipschitz constant satisfies formula (8.36) for $\zeta([x, r])=\xi(x) r^{2}$.

Lemma 8.10 For $\xi: X \rightarrow \mathbb{R}$ let $\zeta: \mathfrak{C} \rightarrow \mathbb{R}$ be defined by $\zeta([x, r]):=$ $\xi(x) r^{2}$.

(i) If $\zeta$ is $\mathrm{d}_{\mathfrak{C}-\text { Lipschitz in }} \mathfrak{C}[R]$, then $\xi \in \operatorname{Lip}_{b}(X)$ with

$$
\begin{aligned}
\sup _{X}|\xi| & \leq \frac{1}{R^{2}} \sup _{\mathfrak{C}[R]}|\zeta| \leq \frac{1}{R} \operatorname{Lip}(\zeta, \mathfrak{C}[R]) \text { and } \\
\operatorname{Lip}(\xi, X) & \leq \frac{1}{R} \operatorname{Lip}(\zeta, \mathfrak{C}[R]) .
\end{aligned}
$$

(ii) If $\xi \in \operatorname{Lip}_{b}(X)$, then $\zeta$ is $\mathrm{d}_{\mathfrak{C}}$-Lipschitz in $\mathfrak{C}[R]$ for every $R>0$ with

$$
\begin{aligned}
\sup _{\mathfrak{C}[R]}|\zeta| & \leq R^{2} \sup _{X}|\xi| \text { and } \\
\operatorname{Lip}^{2}(\zeta, \mathfrak{C}[R]) & \leq R^{2}\left(\operatorname{Lip}^{2}(\xi,(X, \tilde{\mathrm{d}}))+4 \sup _{X}|\xi|^{2}\right),
\end{aligned}
$$

where $\tilde{\mathrm{d}}:=2 \sin \left(\mathrm{d}_{\pi} / 2\right)$.

(iii) In the cases (i) or (ii) we have, for every $x \in X$ and $r \geq 0$, the relation

$$
\left|\mathrm{D}_{\mathfrak{C}} \zeta\right|_{a}^{2}([x, r])=\left\{\begin{array}{cl}
\left(\left|\mathrm{D}_{X} \xi\right|_{a}^{2}(x)+4 \xi^{2}(x)\right) r^{2} & \text { for } r>0, \\
0 & \text { for } r=0 .
\end{array}\right.
$$

The analogous formula holds for the metric slope $\left|\mathrm{D}_{\mathfrak{C}} \zeta\right|([x, r])$. Moreover, equation (8.43) remains true if $\mathrm{d}_{\mathfrak{C}}$ is replaced by the distance $\mathrm{d}_{\pi / 2, \mathfrak{C}}$. 
Proof As usual we set $\mathfrak{y}_{i}=\left[x_{i}, r_{i}\right]$ and $\mathfrak{y}=[x, r]$.

Let us first check statement (i). If $\zeta$ is locally Lipschitz then $|\xi(x)|=$ $\frac{1}{R^{2}}|\zeta([x, R])-\zeta([x, 0])| \leq \frac{1}{R} \operatorname{Lip}(\zeta ; \mathfrak{C}[R])$ for every $R$ sufficiently small, so that $\xi$ is uniformly bounded. Moreover, using (7.4) for every $R>0$ we have

$$
\begin{aligned}
R^{2}\left|\xi\left(x_{1}\right)-\xi\left(x_{2}\right)\right| & \leq\left|\zeta\left(x_{1}, R\right)-\zeta\left(x_{2}, R\right)\right| \leq \operatorname{Lip}(\zeta ; \mathfrak{C}[R]) R \tilde{\mathrm{d}}\left(x_{1}, x_{2}\right) \\
& \leq \operatorname{Lip}(\zeta ; \mathfrak{C}[R]) R \mathrm{~d}\left(x_{1}, x_{2}\right)
\end{aligned}
$$

so that $\xi$ is uniformly Lipschitz and (8.41) holds.

Concerning (ii), for $\xi \in \operatorname{Lip}_{b}(X)$ we set $S:=\sup |\xi|$ and $L:=$ $\operatorname{Lip}(\xi,(X, \tilde{\mathrm{d}}))$ and use the identity

$$
\begin{aligned}
\zeta\left(\mathfrak{y}_{1}\right)-\zeta\left(\mathfrak{y}_{2}\right)= & \left(\xi\left(x_{1}\right)-\xi\left(x_{2}\right)\right) r_{1} r_{2}+2 \xi(x) r\left(r_{1}-r_{2}\right) \\
& +\omega\left(\mathfrak{y}_{1}, \mathfrak{y}_{2} ; \mathfrak{y}\right)\left(r_{1}-r_{2}\right),
\end{aligned}
$$

where $\omega\left(\mathfrak{y}_{1}, \mathfrak{y}_{2} ; \mathfrak{y}\right):=r_{1} \xi\left(x_{1}\right)+r_{2} \xi\left(x_{2}\right)-2 r \xi(x)$ with $\lim _{\mathfrak{y}_{1}, \mathfrak{y}_{2} \rightarrow \mathfrak{y}} \omega\left(\mathfrak{y}_{1}\right.$, $\left.\mathfrak{y}_{2} ; \mathfrak{y}\right)=0$. Since $\left|\omega\left(\mathfrak{y}_{1}, \mathfrak{y}_{2} ; 0\right)\right| \leq 2 R S$ if $\mathfrak{y}_{i} \in \mathfrak{C}[R]$, equation (8.44) with $r=0$ yields

$$
\begin{aligned}
\left|\zeta\left(\mathfrak{y}_{1}\right)-\zeta\left(\mathfrak{y}_{2}\right)\right| & \leq L \tilde{\mathrm{d}}\left(x_{1}, x_{2}\right) r_{1} r_{2}+2 R S\left|r_{1}-r_{2}\right| \\
& \leq\left(L^{2}+4 S^{2}\right)^{1 / 2} R \mathrm{~d}_{\mathfrak{C}}\left(\mathfrak{y}_{1}, \mathfrak{y}_{2}\right) .
\end{aligned}
$$

Letting $R \downarrow 0$ the inequality above also proves (8.43) in the case $r=0$.

In order to prove (8.43) when $r \neq 0$ let us set $L_{\mathfrak{C}}:=\left|\mathrm{D}_{\mathfrak{C}} \zeta\right|_{a}^{2}([x, r])$, $L_{X}:=\left|\mathrm{D}_{X} \xi\right|_{a}(x)$, and let $G_{L}$ be a function satisfying (8.38) with respect to the distance $\tilde{\mathrm{d}}$ (see Remark 8.9). Equation (8.44) yields, for all $\mathfrak{y}=[x, r]$, the relation

$$
\begin{aligned}
\left|\zeta\left(\mathfrak{y}_{1}\right)-\zeta\left(\mathfrak{y}_{2}\right)\right| & \leq G_{L}\left(x_{1}, x_{2}\right) \tilde{\mathrm{d}}\left(x_{1}, x_{2}\right) r_{1} r_{2}+\left(2|\xi(x)| r+\left|\omega\left(\mathfrak{y}_{1}, \mathfrak{y}_{2} ; \mathfrak{y}\right)\right|\right)\left|r_{1}-r_{2}\right| \\
& \leq\left(G_{L}^{2}\left(x_{1}, x_{2}\right) r_{1} r_{2}+\left(2|\xi(x)| r+\left|\omega\left(\mathfrak{y}_{1}, \mathfrak{y}_{2} ; \mathfrak{y}\right)\right|\right)^{2}\right)^{1 / 2} \mathrm{~d}_{\mathfrak{C}}\left(\mathfrak{y}_{1}, \mathfrak{y}_{2}\right) .
\end{aligned}
$$

Passing to the limit $\mathfrak{y}_{1}, \mathfrak{y}_{2} \rightarrow \mathfrak{y}$ and using the fact that $x_{1}, x_{2} \rightarrow x$ due to $r \neq 0$, we obtain $L_{\mathfrak{C}} \leq r\left(L_{X}^{2}+4|\xi(x)|^{2}\right)^{1 / 2}$.

In order to prove the converse inequality we observe that for every $L^{\prime}<L_{X}$ there exist two sequences of points $\left(x_{i, n}\right)_{n \in \mathbb{N}}$ converging to $x$ w.r.t. $\mathrm{d}$ such that $\xi\left(x_{1, n}\right)-\xi\left(x_{2, n}\right) \geq L^{\prime} \delta_{n}$ where $0<\delta_{n}:=\tilde{\mathrm{d}}\left(x_{1, n}, x_{2, n}\right) \rightarrow 0$. Choosing $r_{1, n}:=r$ and $r_{2, n}=r\left(1+\lambda \delta_{n}\right)$ for an arbitrary constant $\lambda \in \mathbb{R}$ with the same sign as $\xi(x)$, we can apply (8.44) and arrive at 


$$
\begin{aligned}
L_{\mathfrak{C}} & \geq \liminf _{n \rightarrow \infty} \frac{\left|\zeta\left(\mathfrak{y}_{1, n}\right)-\zeta\left(\mathfrak{y}_{2, n}\right)\right|}{\mathrm{d}_{\mathfrak{C}}\left(\mathfrak{y}_{1, n}, \mathfrak{y}_{2, n}\right)} \geq \liminf _{n \rightarrow \infty} \frac{L^{\prime} \delta_{n} r^{2}+2|\xi(x)| r^{2}|\lambda| \delta_{n}+o\left(\delta_{n}\right)}{\sqrt{\lambda^{2} r^{2} \delta_{n}^{2}+r^{2} \delta_{n}^{2}+o\left(\delta_{n}\right)}} \\
& =r \frac{L^{\prime}+2|\xi(x)||\lambda|}{\sqrt{\lambda^{2}+1}} .
\end{aligned}
$$

Optimizing with respect to $\lambda$ we obtain

$$
L_{\mathfrak{C}}^{2} \geq r^{2}\left(\left(L^{\prime}\right)^{2}+4|\xi(x)|^{2}\right), \text { where } L^{\prime} \leq L_{X} \text { is arbitrary. }
$$

This proves (8.43) for the asymptotic Lipschitz constant $\left|\mathrm{D}_{\mathfrak{C}} \zeta\right|_{a}$. The arguments for proving (8.43) for metric slopes $\left|\mathrm{D}_{\mathfrak{C}} \zeta\right|$ are completely analogous.

\section{Hopf-Lax formula and subsolutions to metric Hamilton-Jacobi equation} in the cone $\mathfrak{C}$. Whenever $f \in \operatorname{Lip}_{b}(\mathfrak{C})$ the Hopf-Lax formula

$$
\mathscr{Q}_{t} f(\mathfrak{y}):=\inf _{\mathfrak{y}^{\prime} \in \mathfrak{C}^{\mathfrak{f}}}\left(f\left(\mathfrak{y}^{\prime}\right)+\frac{1}{2 t} \mathrm{~d}_{\mathfrak{C}^{\mathfrak{C}}}^{2}\left(\mathfrak{y}, \mathfrak{y}^{\prime}\right)\right) \text { for } \mathfrak{y} \in \mathfrak{C} \text { and } t>0,
$$

provides a function $t \mapsto \mathscr{Q}_{t} f$ which is Lipschitz from $[0, \infty)$ to $\mathrm{C}_{b}(\mathfrak{C})$, satisfies the a-priori bounds

$$
\inf _{\mathfrak{C}} f \leq \mathscr{Q}_{t} f \leq \sup _{\mathfrak{C}} f, \quad \operatorname{Lip}\left(\mathscr{Q}_{t} f ; \mathfrak{C}\right) \leq 2 \operatorname{Lip}(f, \mathfrak{C})
$$

and solves

$$
\partial_{t}^{+} \mathscr{Q}_{t} f(\mathfrak{z})+\frac{1}{2}\left|\mathrm{D}_{\mathfrak{C}} \mathscr{Q}_{t} f\right|_{a}^{2}(\mathfrak{z}) \leq 0 \text { for every } \mathfrak{z} \in \mathfrak{C}, t>0,
$$

where $\partial_{t}^{+}$denotes the partial right derivative w.r.t. $t$.

It is also possible to prove that for every $\mathfrak{y} \in \mathfrak{C}$ the time derivative of $\mathscr{Q}_{t} f(\mathfrak{y})$ exists with possibly countable exceptions and that (8.47) is in fact an equality if $\left(\mathfrak{C}, \mathrm{d}_{\mathfrak{C}}\right)$ is a length space, a property that always holds if $(X, \mathrm{~d})$ is a length metric space. This is stated in our main result:

Theorem 8.11 (Metric subsolution of Hamilton-Jacobi equation in $X$ ) Let $\xi \in \operatorname{Lip}_{b}(X)$ satisfy the uniform lower bound $P:=1+2 \inf _{X}(\xi \wedge 0)>0$ and let us set $\zeta([x, r]):=\xi(x) r^{2}$. Then, for every $t \in[0,1]$ we have

$$
\begin{aligned}
\mathscr{Q}_{t} \zeta([x, r]) & =\xi_{t}(x) r^{2}, \text { where } \xi_{t}(x):=\mathscr{P}_{t} \xi(x) \text { and } \\
\mathscr{P}_{t} \xi(x) & :=\inf _{x^{\prime} \in X}\left(\frac{\xi\left(x^{\prime}\right)}{1+2 t \xi\left(x^{\prime}\right)}+\frac{\sin ^{2}\left(\mathrm{~d}_{\pi / 2}\left(x, x^{\prime}\right)\right)}{2 t\left(1+2 t \xi\left(x^{\prime}\right)\right)}\right) \\
& =\inf _{x^{\prime} \in X} \frac{1}{2 t}\left(1-\frac{\cos ^{2}\left(\mathrm{~d}_{\pi / 2}\left(x, x^{\prime}\right)\right)}{1+2 t \xi\left(x^{\prime}\right)}\right) .
\end{aligned}
$$


Moreover, for every $R>0$ we have

$$
\begin{aligned}
& \xi_{t}(x) r^{2}= \inf _{\substack{\mathfrak{y}^{\prime}=\left[x^{\prime}, r^{\prime}\right] \in \mathfrak{C}[R] \\
\\
\text { for all } x \in X, r \leq P R .}}\left(\xi\left(x^{\prime}\right)\left(r^{\prime}\right)^{2}+\frac{1}{2 t} \mathrm{~d}_{\mathfrak{C}}^{2}\left([x, r] ;\left[x^{\prime}, r^{\prime}\right]\right)\right) \\
&
\end{aligned}
$$

The map $t \mapsto \xi_{t}$ is Lipschitz from $[0,1]$ to $\mathrm{C}_{b}(X)$ with $\xi_{t} \in \operatorname{Lip}_{b}(X)$ for every $t \in[0,1]$. Moreover, $\xi_{t}$ is a subsolution to the generalized Hamilton-Jacobi equation

$$
\partial_{t}^{+} \xi_{t}(x)+\frac{1}{2}\left|\mathrm{D}_{X} \xi_{t}\right|_{a}^{2}(x)+2 \xi_{t}^{2}(x) \leq 0 \text { for } x \in X \text { and } t \in[0,1] .
$$

For every $x \in X$ the map $t \mapsto \xi_{t}(x)$ is time differentiable with at most countable exceptions. If $(X, \mathrm{~d})$ is a length space, (8.50a) holds with equality and $\left|\mathrm{D}_{X} \xi_{t}\right|_{a}(x)=\left|\mathrm{D}_{X} \xi_{t}\right|(x)$ for every $x \in X$ and $t \in[0,1]$ :

$$
\partial_{t}^{+} \xi_{t}(x)+\frac{1}{2}\left|\mathrm{D}_{X} \xi_{t}\right|_{a}^{2}(x)+2 \xi_{t}^{2}(x)=0, \quad\left|\mathrm{D}_{X} \xi_{t}\right|_{a}(x)=\left|\mathrm{D}_{X} \xi_{t}\right|(x) .
$$

Notice that when $\xi(x) \equiv \xi$ is constant, (8.48) reduces to $\mathscr{P}_{t} \xi=\xi /(1+2 t \xi)$ which is the solution to the elementary differential equation $\frac{\mathrm{d}}{\mathrm{d} t} \xi+2 \xi^{2}=0$. Proof Let us observe that $\inf _{t \in[0,1], z \in X}(1+2 t \xi(z))=P>0$. A simple calculation shows

$$
\begin{aligned}
& \xi\left(x^{\prime}\right)\left(r^{\prime}\right)^{2}+\frac{1}{2 t} \mathrm{~d}_{\mathfrak{C}}^{2}\left([x, r] ;\left[x^{\prime}, r^{\prime}\right]\right) \\
&=\frac{1}{2 t}\left(\left(1+2 t \xi\left(x^{\prime}\right)\right)\left(r^{\prime}\right)^{2}+r^{2}-2 r r^{\prime} \cos \left(\mathrm{d}_{\pi}\left(x, x^{\prime}\right)\right)\right) \\
&=\frac{1}{2 t\left(1+2 t \xi\left(x^{\prime}\right)\right)}\left[\left(\left(1+2 t \xi\left(x^{\prime}\right)\right) r^{\prime}-\cos \left(\mathrm{d}_{\pi}\left(x, x^{\prime}\right)\right) r\right)^{2}\right. \\
&\left.+r^{2}\left(2 t \xi\left(x^{\prime}\right)+\sin ^{2}\left(\mathrm{~d}_{\pi}\left(x, x^{\prime}\right)\right)\right)\right] .
\end{aligned}
$$

Hence, if we choose

$$
r^{\prime}:= \begin{cases}r \cos \left(\mathrm{d}_{\pi}\left(x, x^{\prime}\right)\right) /\left(1+2 t \xi\left(x^{\prime}\right)\right) & \text { if } \mathrm{d}\left(x, x^{\prime}\right) \leq \pi / 2 \\ 0 & \text { otherwise, }\end{cases}
$$

we find (notice the truncation at $\pi / 2$ instead of $\pi$ )

$$
\begin{aligned}
& \inf _{r^{\prime} \geq 0} \xi\left(x^{\prime}\right)\left(r^{\prime}\right)^{2}+\frac{1}{2 t} \mathrm{~d}_{\mathfrak{C}}^{2}\left([x, r] ;\left[x^{\prime}, r^{\prime}\right]\right) \\
& \quad=\frac{r^{2}}{2 t\left(1+2 t \xi\left(x^{\prime}\right)\right)}\left(2 t \xi\left(x^{\prime}\right)+\sin ^{2}\left(\mathrm{~d}_{\pi / 2}\left(x, x^{\prime}\right)\right)\right),
\end{aligned}
$$


which yields (8.48). Now (8.49) also follows, since $r^{\prime} \leq r / P$ in (8.51).

Equation (8.49) also shows that the function $\zeta_{t}=\xi_{t}(x) r^{2}$ coincides on $\mathfrak{C}[P R]$ with the solution $\zeta_{t}^{R}$ given by the Hopf-Lax formula in the metric space $\mathfrak{C}[R]$. Since the initial datum $\zeta$ is bounded and Lipschitz on $\mathfrak{C}[R]$ we deduce that $\zeta_{t}^{R}$ is bounded and Lipschitz, so that $t \mapsto \xi_{t}$ is bounded and Lipschitz in $X$ by Lemma 8.10 .

Equation (8.50a) and the other regularity properties then follow by (8.43) and the general properties of the Hopf-Lax formula in $\mathfrak{C}[R]$.

\section{Duality between the Hellinger-Kantorovich distance and subsolutions to} the generalized Hamilton-Jacobi equation. We conclude this section with the main application of the above results to the Hellinger-Kantorovich distance.

Theorem 8.12 Let us suppose that $(X, \mathrm{~d})$ is a complete and separable metric space.

(i) If $\mu \in \mathrm{AC}^{2}([0,1] ;(\mathcal{N}(X), \mathrm{H}))$ and $\xi:[0,1] \rightarrow \operatorname{Lip}_{b}(X)$ is uniformly bounded, Lipschitz w.r.t. the uniform norm, and satisfies (8.50a), then the curve $t \mapsto \int \xi_{t} \mathrm{~d} \mu_{t}$ is absolutely continuous and

$$
\frac{\mathrm{d}}{\mathrm{d} t} \int_{X} \xi_{t} \mathrm{~d} \mu_{t} \leq \frac{1}{2}\left|\mu_{t}^{\prime}\right|_{\mathrm{HK}}^{2}
$$

(ii) If $(X, \mathrm{~d})$ is a length space, then for every $\mu_{0}, \mu_{1}$ and $k \in \mathbb{N} \cup\{\infty\}$ we have

$$
\begin{array}{r}
\frac{1}{2} \mathrm{H}^{2}\left(\mu_{0}, \mu_{1}\right)=\sup \left\{\int_{X} \xi_{1} \mathrm{~d} \mu_{1}-\int_{X} \xi_{0} \mathrm{~d} \mu_{0}: \xi \in \mathrm{C}^{k}\left([0,1] ; \operatorname{Lip}_{b}(X)\right),\right. \\
\left.\partial_{t} \xi_{t}(x)+\frac{1}{2}\left|\mathrm{D}_{X} \xi_{t}\right|^{2}(x)+2 \xi_{t}^{2}(x) \leq 0 \text { in } X \times(0,1)\right\} .
\end{array}
$$

Moreover, in the above formula we can also take the supremum over functions $\xi \in \mathrm{C}^{k}\left([0,1] ; \operatorname{Lip}_{b}(X)\right)$ with bounded support.

Proof If $\xi$ satisfies (8.50a) then setting $\zeta_{t}([x, r]):=\xi_{t}(x) r^{2}$ we obtain a family of functions $t \mapsto \zeta_{t}, t \in[0,1]$, whose restriction to every $\mathfrak{C}[R]$ is uniformly bounded and Lipschitz, and it is Lipschitz continuous with respect to the uniform norm of $\mathrm{C}_{b}(\mathfrak{C}[R])$. By Lemma 8.10 the function $\zeta$ solves

$$
\partial_{t}^{+} \zeta_{t}+\frac{1}{2}\left|D_{\mathfrak{C}} \zeta_{t}\right|_{a}^{2} \leq 0 \quad \text { in } \mathfrak{C} \times(0,1) .
$$


According to Theorem 8.4 we find $\theta>0$ and a curve $\alpha \in \mathrm{AC}^{2}\left([0,1] ;\left(\mathcal{P}_{2}(\mathfrak{C}[\theta])\right.\right.$, $\left.\mathrm{W}_{\mathrm{d}_{\mathfrak{C}}}\right)$ ) satisfying (8.22). Applying the results of [6, Sect.6], the map $t \mapsto$ $\int_{\mathfrak{C}} \zeta_{t} \mathrm{~d} \alpha_{t}$ is absolutely continuous with

$$
\frac{\mathrm{d}}{\mathrm{d} t} \int_{\mathfrak{C}} \zeta_{t} \mathrm{~d} \alpha_{t} \leq \frac{1}{2}\left|\alpha_{t}^{\prime}\right|_{\mathrm{W}_{\mathrm{d}_{\mathfrak{C}}}}^{2} \mathscr{L}^{1} \text {-a.e. in }(0,1) .
$$

Since $\int_{\mathfrak{C}} \zeta_{t} \mathrm{~d} \alpha_{t}=\int_{X} \xi_{t} \mathrm{~d} \mu_{t}$ we obtain (8.53).

Let us now prove (ii). As a first step, denoting by $S$ the right-hand side of (8.54), we prove that $\mathrm{HK}^{2}\left(\mu_{0}, \mu_{1}\right) \geq S$. If $\xi \in \mathrm{C}^{1}\left([0,1]\right.$; $\left.\operatorname{Lip}_{b}(X)\right)$ satisfies the pointwise inequality

$$
\partial_{t} \xi_{t}(x)+\frac{1}{2}\left|\mathrm{D}_{X} \xi_{t}\right|^{2}(x)+2 \xi_{t}^{2}(x) \leq 0,
$$

then it also satisfies ( $8.50 \mathrm{a})$, because (8.55) provides the relation

$$
\frac{1}{2}\left|\mathrm{D}_{X} \xi_{t}\right|^{2}(x) \leq-\left(\partial_{t} \xi_{t}(x)+2 \xi_{t}^{2}(x)\right) \text { for every }(x, t) \in X \times(0,1),
$$

where the right hand side is bounded and continuous in $X$. Equation (8.56) thus yields the same inequality for the upper semicontinuous envelope of $\left|D_{X} \xi_{t}\right|$ and this function coincides with $\left|\mathrm{D}_{X} \xi_{t}\right|_{a}$ since $X$ is a length space.

We can therefore apply the previous point (i) by choosing $\lambda>1$ and a Lipschitz curve $\mu:[0,1] \rightarrow \mathcal{M}(X)$ joining $\mu_{0}$ to $\mu_{1}$ with metric velocity $\left|\mu_{t}^{\prime}\right|_{\mathrm{HK}} \leq \lambda \mathrm{HK}\left(\mu_{0}, \mu_{1}\right)$, whose existence is guaranteed by the length property of $X$ and a standard rescaling technique. Relation (8.53) yields

$$
2 \int_{X} \xi_{1} \mathrm{~d} \mu_{1}-2 \int_{X} \xi_{0} \mathrm{~d} \mu_{0} \leq \int_{0}^{1}\left|\mu_{t}^{\prime}\right|_{\mathrm{HK}}^{2} \mathrm{~d} t \leq \lambda^{2} \mathrm{HK}^{2}\left(\mu_{0}, \mu_{1}\right) .
$$

Since $\lambda>1$ is arbitrary, we get $\mathrm{HK}^{2}\left(\mu_{0}, \mu_{1}\right) \geq S$.

In order to prove the converse inequality in (8.54) we fix $\eta>0$ and apply the duality Theorem 7.21 to get $\xi_{0} \in \operatorname{Lip}_{b s}(X)$ (the space of Lipschitz functions with bounded support) with inf $\xi_{0}>-1 / 2$ such that

$$
2 \int_{X} \mathscr{P}_{1} \xi_{0} \mathrm{~d} \mu_{1}-2 \int_{X} \xi_{0} \mathrm{~d} \mu_{0} \geq \mathrm{H}^{2}\left(\mu_{0}, \mu_{1}\right)-\eta .
$$

Setting $\xi_{t}:=\mathscr{P}_{t} \xi_{0}$ we find a solution to (8.50a) which has bounded support, is uniformly bounded in $\operatorname{Lip}_{b}(X)$ and Lipschitz with respect to the uniform norm. We have to show that $\left(\xi_{t}\right)_{t \in[0,1]}$ can be suitably approximated by smoother solutions $\xi^{\varepsilon} \in \mathrm{C}^{\infty}\left([0,1] ; \operatorname{Lip}_{b}(X)\right), \varepsilon>0$, in such a way that $\int \xi_{i}^{\varepsilon} \mathrm{d} \mu_{i} \rightarrow$ $\int \xi_{i} \mathrm{~d} \mu_{i}$ as $\varepsilon \downarrow 0$ for $i=0,1$. 
We use an argument of [1], which relies on the scaling invariance of the generalized Hamilton-Jacobi equation: If $\xi$ solves (8.55) and $\lambda>0$, then $\xi_{t}^{\lambda}(x):=\lambda \xi_{\lambda t+t_{0}}(x)$ solves (8.55) as well. Hence, by approximating $\xi_{t}$ with $\lambda \xi(\lambda t+(1-\lambda) / 2, x)$ with $0<\lambda<1$ and passing to the limit $\lambda \uparrow 1$, it is not restrictive to assume that $\xi$ is defined in a larger interval $[a, b]$, with $a<0, b>1$. Now, a time convolution is well defined on [0, 1], for which we use a symmetric, nonnegative kernel $\kappa \in \mathrm{C}_{\mathrm{c}}^{\infty}(\mathbb{R})$ with integral 1 defined via

$$
\xi_{t}^{\varepsilon}(x):=\left(\xi_{(\cdot)}(x) * \kappa_{\varepsilon}\right)_{t}=\int_{\mathbb{R}} \xi_{w}(x) \kappa_{\varepsilon}(t-w) \mathrm{d} w,
$$

where $\kappa_{\varepsilon}(t):=\varepsilon^{-1} \kappa(t / \varepsilon)$. It yields a curve $\xi^{\varepsilon} \in \mathrm{C}^{\infty}\left([0,1] ; \operatorname{Lip}_{b}(X)\right)$ satisfying

$$
\partial_{t} \xi_{t}^{\varepsilon}+\frac{1}{2}\left(\left|\mathrm{D}_{X} \xi_{(\cdot)}\right|^{2}\right) * \kappa_{\varepsilon}+2\left(\xi_{(\cdot)}^{2}\right) * \kappa_{\varepsilon} \leq 0 \quad \text { in } X \times[0,1] .
$$

By Jensen's inequality, we have the two estimates $\xi_{(\cdot)}^{2} * \kappa_{\varepsilon} \geq\left(\xi_{(\cdot)} * \kappa_{\varepsilon}\right)^{2}$ and $\left|\mathrm{D}_{X} \xi_{(\cdot)}\right|^{2} * \kappa_{\varepsilon} \geq\left(\left|\mathrm{D}_{X} \xi_{(\cdot)}\right| * \kappa_{\varepsilon}\right)^{2}$. Moreover, applying the following Lemma 8.13 we also get $\left|\mathrm{D}_{X} \xi_{(\cdot)}\right| * \kappa_{\varepsilon} \geq\left|\mathrm{D}_{X} \xi_{(\cdot)}^{\varepsilon}\right|$, so that the smooth convolution $\xi_{t}^{\varepsilon}$ satisfies (8.55). Since $\xi_{t}^{\varepsilon} \rightarrow \xi_{t}$ uniformly in $X$ for every $t \in[0,1]$, we easily get

$$
S \geq \lim _{\varepsilon \downarrow 0} 2\left(\int_{X} \xi_{1}^{\varepsilon} \mathrm{d} \mu_{1}-\int_{X} \xi_{0}^{\varepsilon} \mathrm{d} \mu_{0}\right) \geq \mathrm{H}^{2}\left(\mu_{0}, \mu_{1}\right)-\eta .
$$

Since $\eta>0$ is arbitrary the proof of (ii) is complete.

The next result shows that averaging w.r.t. a probability measure $\pi \in \mathcal{P}(\Omega)$ does not increase the metric slope nor the asymptotic Lipschitz constant. This was used in the last proof for the temporal smoothing and will be used for spatial smoothing in Corollary 8.14.

Lemma 8.13 Let $(X, \mathrm{~d})$ be a separable metric space, let $(\Omega, \mathcal{B}, \pi)$ be a probability space (i.e. $\pi(\Omega)=1$ ) and let $\xi_{\omega} \in \operatorname{Lip}_{b}(X), \omega \in \Omega$, be a family of uniformly bounded functions such that $\sup _{\omega \in \Omega} \operatorname{Lip}\left(\xi_{\omega} ; X\right)<\infty$ and $\omega \mapsto \xi_{\omega}(x)$ is $\mathcal{B}$-measurable for every $x \in X$. Then the function $x \mapsto \xi(x):=\int_{\Omega} \xi_{\omega}(x) \mathrm{d} \pi(\omega)$ belongs to $\operatorname{Lip}_{b}(X)$ and for every $x \in X$ the maps $\omega \mapsto\left|\mathrm{D}_{X} \xi_{\omega}\right|(x)$ and $\omega \mapsto\left|\mathrm{D}_{X} \xi_{\omega}\right|_{a}(x)$ are $\mathcal{B}$-measurable and satisfy

$$
\left|\mathrm{D}_{X} \xi\right|_{a}(x) \leq \int_{X}\left|\mathrm{D}_{X} \xi_{\omega}\right|_{a}(x) \mathrm{d} \pi(\omega), \quad\left|\mathrm{D}_{X} \xi\right|(x) \leq \int_{X}\left|\mathrm{D}_{X} \xi_{\omega}\right|(x) \mathrm{d} \pi(\omega) .
$$

Proof The fact that $\xi \in \operatorname{Lip}_{b}(X)$ is obvious. To show measurability we fix $x \in X$ and use the expression (8.37) for $\left|\mathrm{D}_{X} \xi\right|_{a}(x)$. It is sufficient to prove that 
for every $r>0$ the map $\omega \mapsto s_{r, \omega}(x):=\sup _{y \neq z \in B_{r}(x)}\left|\xi_{\omega}(y)-\xi_{\omega}(z)\right| / \mathrm{d}(y, z)$ is $\mathcal{B}$-measurable. This property follows by the continuity of $\xi_{\omega}$ and the separability of $X$, so that it is possible to restrict the supremum to a countable dense collection of points $\tilde{B}_{r}(x)$ in $B_{r}(x)$. Thus, the measurability follows, because the pointwise supremum of countably many measurable functions is measurable. An analogous argument holds for $\left|\mathrm{D}_{X} \xi_{\omega}\right|$.

Using the definition $\xi:=\int \xi_{\omega} \mathrm{d} \pi$ we have

$$
\frac{|\xi(y)-\xi(z)|}{\mathrm{d}(y, z)} \leq \int_{\Omega} \frac{\left|\xi_{\omega}(y)-\xi_{\omega}(z)\right|}{\mathrm{d}(y, z)} \mathrm{d} \pi(\omega) \text { for } y \neq z .
$$

Taking the supremum with respect to $y, z \in \tilde{B}_{r}(x)$ and $y \neq z$, we obtain

$$
\sup _{y \neq z \in B_{r}(x)} \frac{|\xi(y)-\xi(z)|}{\mathrm{d}(y, z)} \leq \int_{\Omega} s_{r, \omega}(x) \mathrm{d} \pi(\omega) .
$$

A further limit as $r \downarrow 0$ and the application of the Lebesgue Dominated convergence Theorem yields the first inequality of (8.59). The argument to prove the second inequality is completely analogous.

When $X=\mathbb{R}^{d}$ the characterization (8.54) of $\mathrm{HK}$ holds for an even smoother class of subsolutions $\xi$ of the generalized Hamilton-Jacobi equation.

Corollary 8.14 Let $X=\mathbb{R}^{d}$ be endowed with the Euclidean distance. Then

$$
\begin{array}{r}
\mathrm{H}^{2}\left(\mu_{0}, \mu_{1}\right)=2 \sup \left\{\int_{X} \xi_{1} \mathrm{~d} \mu_{1}-\int_{X} \xi_{0} \mathrm{~d} \mu_{0}: \xi \in \mathrm{C}_{\mathrm{c}}^{\infty}\left(\mathbb{R}^{d} \times[0,1]\right),\right. \\
\left.\partial_{t} \xi_{t}(x)+\frac{1}{2}\left|\mathrm{D}_{x} \xi_{t}(x)\right|^{2}+2 \xi_{t}^{2}(x) \leq 0 \text { in } X \times(0,1)\right\} .
\end{array}
$$

Proof We just have to check that the supremum of (8.54) does not change if we substitute $\mathrm{C}^{\infty}\left([0,1] ; \operatorname{Lip}_{b s}\left(\mathbb{R}^{d}\right)\right)$ with $\mathrm{C}_{\mathrm{c}}^{\infty}\left(\mathbb{R}^{d} \times[0,1]\right)$. This can be achieved by approximating any subsolution $\xi \in \mathrm{C}^{\infty}\left([0,1] ; \operatorname{Lip}_{b s}\left(\mathbb{R}^{d}\right)\right)$ via convolution in space with a smooth kernel with compact support, which still provides a subsolution thanks to Lemma 8.13.

\subsection{The dynamic interpretation of the Hellinger-Kantorovich distance "à la Benamou-Brenier"}

In this section we will apply the superposition principle of Theorem 8.4 and the duality result 8.12 with subsolutions of the Hamilton-Jacobi equation to quickly derive a dynamic formulation "à la Benamou-Brenier" [7,37], [2, 
Sect. 8] of the Hellinger-Kantorovich distance, which has also been considered in the recent [27]. In order to keep the exposition simpler, we will consider the case $X=\mathbb{R}^{d}$ with the canonical Euclidean distance $\mathrm{d}\left(x_{1}, x_{2}\right):=\left|x_{1}-x_{2}\right|$, but the result can be extended to more general Riemannian and metric settings, e.g. arguing as in [6, Sect. 6]. A different approach, based on suitable representation formulae for the continuity equation, is discussed in our companion paper [30].

Our starting point is provided by a suitable class of linear continuity equations with reaction. In the following we will denote by $\mu_{I} \in \mathcal{N}\left(\mathbb{R}^{d} \times[0,1]\right)$ the measure

$$
\int \xi \mathrm{d} \mu_{I}:=\int_{0}^{1} \int_{\mathbb{R}^{d}} \xi_{t}(x) \mathrm{d} \mu_{t}(x) \mathrm{d} t
$$

induced by a curve $\mu \in \mathrm{C}^{0}\left([0,1] ; \mathcal{M}\left(\mathbb{R}^{d}\right)\right)$.

Definition 8.15 Let $\mu \in \mathrm{C}^{0}\left([0,1] ; \mathcal{M}\left(\mathbb{R}^{d}\right)\right)$, let $(\boldsymbol{v}, w): \mathbb{R}^{d} \times(0,1) \rightarrow \mathbb{R}^{d+1}$ be a Borel vector field in $\mathrm{L}^{2}\left(\mathbb{R}^{d} \times(0,1), \mu_{I} ; \mathbb{R}^{d+1}\right)$, thus satisfying

$$
\int_{0}^{1} \int_{\mathbb{R}^{d}}\left(\left|\boldsymbol{v}_{t}(x)\right|^{2}+w_{t}^{2}(x)\right) \mathrm{d} \mu_{t}(x) \mathrm{d} t=\int|(\boldsymbol{v}, w)|^{2} \mathrm{~d} \mu_{I}<\infty .
$$

We say that $\mu$ satisfies the continuity equation with reaction governed by $(\boldsymbol{v}, w)$ if

$$
\partial_{t} \mu_{t}+\nabla \cdot\left(\boldsymbol{v}_{t} \mu_{t}\right)=w_{t} \mu_{t} \text { holds in } \mathscr{D}^{\prime}\left(\mathbb{R}^{d} \times(0,1)\right),
$$

i.e. for every test function $\xi \in \mathrm{C}_{\mathrm{c}}^{\infty}\left(\mathbb{R}^{d} \times(0,1)\right)$

$$
\int_{0}^{1} \int_{\mathbb{R}^{d}}\left(\partial_{t} \xi_{t}(x)+\mathrm{D}_{x} \xi_{t}(x) \boldsymbol{v}_{t}(x)+\xi_{t}(x) w_{t}(x)\right) \mathrm{d} \mu_{t} \mathrm{~d} t=0
$$

An equivalent formulation [2, Sect. 8.1] of (8.63) is

$$
\frac{\mathrm{d}}{\mathrm{d} t} \int_{\mathbb{R}^{d}} \xi(x) \mathrm{d} \mu_{t}(x)=\int_{\mathbb{R}^{d}}\left(\mathrm{D}_{x} \xi(x) \boldsymbol{v}_{t}(x)+\xi(x) w_{t}(x)\right) \mathrm{d} \mu_{t} \quad \text { in } \mathscr{D}^{\prime}(0,1),
$$

for every $\xi \in \mathrm{C}_{\mathrm{c}}^{\infty}\left(\mathbb{R}^{d}\right)$. We have a first representation result for absolutely continuous curves $t \mapsto \mu_{t}$, which relies in Theorem 8.4, where we constructed suitable lifted plans $\pi \in \mathcal{P}\left(\mathrm{AC}^{2}([0,1] ; \mathfrak{C})\right)$, i.e. $\mu_{t}=\mathfrak{h}_{t}^{2} \boldsymbol{\pi}$, where $\mathfrak{C}$ is now the cone over $\mathbb{R}^{d}$.

Theorem 8.16 Let $\left(\mu_{t}\right)_{t \in[0,1]}$ be a curve in $\mathrm{AC}^{2}\left([0,1] ;\left(\mathcal{M}\left(\mathbb{R}^{d}\right), \mathrm{HK}\right)\right)$. Then $\mu$ satisfies the continuity equation with reaction (8.63) with a Borel vector field $(\boldsymbol{v}, w) \in \mathrm{L}^{2}\left(\mathbb{R}^{d} \times(0,1), \mu_{I} ; \mathbb{R}^{d+1}\right)$ satisfying

$$
\left(\boldsymbol{v}_{t}, w_{t}\right) \in \mathrm{L}^{2}\left(\mathbb{R}^{d} ; \mu_{t}\right), \int\left(\left|\boldsymbol{v}_{t}\right|^{2}+\frac{1}{4}\left|w_{t}\right|^{2}\right) \mathrm{d} \mu_{t} \leq\left|\mu_{t}^{\prime}\right|^{2}
$$

for $\mathscr{L}^{1}$-a.e. $t \in(0,1)$. 
Proof We will denote by $I$ the interval $[0,1]$ endowed with the Lebesgue measure $\lambda=\mathscr{L}^{1}\left\llcorner[0,1]\right.$. Recalling the map $(\mathrm{x}, \mathrm{r}): \mathfrak{C} \rightarrow \mathbb{R}^{d} \times[0, \infty)$ we define the maps $\mathrm{x}_{I}: \mathrm{C}(I ; \mathfrak{C}) \times I \rightarrow \mathbb{R}^{d} \times I$ and $\mathrm{R}: \mathrm{C}(I ; \mathfrak{C}) \times I \rightarrow \mathbb{R}_{+}$via $\mathrm{x}_{I}(\mathrm{z}, t):=(\mathrm{x}(\mathrm{z}(t)), t)$ and $\mathrm{R}(\mathrm{z}, t):=\mathrm{r}(\mathrm{z}(t))$.

Let $\boldsymbol{\pi}$ be a dynamic plan in $\mathfrak{C}$ representing $\mu_{t}$ as in Theorem 8.4. We consider the deformed dynamic plan $\pi_{I}:=\left(\mathrm{R}^{2} \pi\right) \otimes \lambda$, the measure $\hat{\mu}_{I}:=\left(\mathrm{x}_{I}\right)_{\sharp} \boldsymbol{\pi}_{I}$ and the disintegration $\left(\tilde{\boldsymbol{\pi}}_{x, t}\right)_{(x, t) \in \mathbb{R}^{d} \times I}$ of $\boldsymbol{\pi}_{I}$ with respect to $\hat{\mu}_{I}$. Since $\pi$ is in fact a dynamic plan on $\mathfrak{C}[\Theta]$, where $\Theta$ is given by (8.21), we notice that $\pi_{I} \leq \Theta^{2}(\boldsymbol{\pi} \otimes \lambda)$ so that $\pi_{I}$ has finite mass and

$$
\hat{\mu}_{I}=\int_{0}^{1}\left(\mu_{t} \otimes \delta_{t}\right) \mathrm{d} \lambda(t),
$$

coincides with $\mu_{I}$ in (8.61), because for every $\xi \in \mathrm{B}_{b}\left(\mathbb{R}^{d} \times I\right)$ we have

$$
\begin{aligned}
\int \xi \mathrm{d} \hat{\mu}_{I} & =\int \xi(\mathbf{x}(\mathrm{z}(t)), t) \mathrm{r}^{2}(\mathrm{z}(t)) \mathrm{d}(\boldsymbol{\pi} \otimes \lambda)(\mathrm{z}, t) \\
& =\int_{0}^{1} \int_{\mathbb{R}^{d}} \xi_{t}(x) \mathrm{d} \mu_{t}(x) \mathrm{d} t=\int \xi \mathrm{d} \mu_{I} .
\end{aligned}
$$

Let $\boldsymbol{u} \in \mathrm{L}^{2}\left(\mathrm{AC}^{2}(I ; \mathfrak{C}) \times I ; \boldsymbol{\pi} \otimes \lambda ; \mathbb{R}^{d+1}\right)$ be the Borel vector field $\boldsymbol{u}(\mathfrak{y}, t):=$ $\mathfrak{y}_{\mathfrak{C}}^{\prime}(t)$ for every curve $\mathfrak{y} \in \operatorname{AC}^{2}(I ; \mathfrak{C})$ and $t \in I$, where $\mathfrak{y}_{\mathfrak{C}}^{\prime}$ is defined as in (8.14). By taking the density of the vector measure $\left(\mathbf{X}_{I}\right)_{\sharp}\left(\boldsymbol{u} \boldsymbol{\pi}_{I}\right)$ with respect to $\mu_{I}$ we obtain a Borel vector field $\boldsymbol{u}_{I}=(\boldsymbol{v}, \hat{w}) \in \mathrm{L}^{2}\left(\mathbb{R}^{d} \times I ; \mu_{I} ; \mathbb{R}^{d+1}\right)$ which satisfies

$$
\begin{aligned}
\boldsymbol{u}_{I}(x, t) & =\int \boldsymbol{u} \mathrm{d} \boldsymbol{\pi}_{x, t} \text { for } \mu_{I} \text {-a.e. }(x, t) \in \mathbb{R}^{d} \times I \text { and } \\
\int\left(\left|\boldsymbol{v}_{t}\right|^{2}+\hat{w}_{t}^{2}\right) \mathrm{d} \mu_{t} & \leq\left|\mu_{t}^{\prime}\right|^{2} .
\end{aligned}
$$

Choosing a test function $\zeta([x, r], t):=\xi(x) \eta(t) r^{2}$ with $\xi \in \mathrm{C}_{\mathrm{c}}^{\infty}\left(\mathbb{R}^{d}\right)$ and $\eta \in \mathrm{C}_{\mathrm{c}}^{\infty}(I)$ we can exploit the chain rule (8.16) in $\mathbb{R}^{d}$ and find

$$
\begin{aligned}
& -\int_{0}^{1} \eta^{\prime} \int_{\mathbb{R}^{d}} \xi \mathrm{d} \mu_{t} \mathrm{~d} t=-\int_{\mathbb{R}^{d} \times I} \eta^{\prime}(t) \xi(x) \mathrm{d} \mu_{I} \\
& =-\int \xi\left(\mathbf{x}(\mathfrak{y}(t)) \mathrm{r}^{2}(\mathfrak{y}(t)) \eta^{\prime}(t) \mathrm{d}(\boldsymbol{\pi} \otimes \lambda)\right. \\
& =-\int \partial_{t} \zeta(\mathfrak{y}(t), t) \mathrm{d}(\boldsymbol{\pi} \otimes \lambda) \\
& =\int\left(-\frac{\mathrm{d}}{\mathrm{d} t} \zeta(\mathfrak{y}(t), t)+\left\langle\mathrm{D}_{\mathfrak{C}} \zeta(\mathfrak{y}(t), t), \mathfrak{y}_{\mathfrak{C}^{\prime}}^{\prime}(t)\right\rangle\right) \mathrm{d}(\boldsymbol{\pi} \otimes \lambda)
\end{aligned}
$$




$$
\begin{aligned}
& =\int\left(\int_{0}^{1}-\frac{\mathrm{d}}{\mathrm{d} t} \zeta(\mathfrak{y}(t), t) \mathrm{d} t\right) \mathrm{d} \boldsymbol{\pi}+\int\left\langle\left(\mathrm{D}_{x} \xi\left(\mathbf{x}_{I}\right), 2 \xi\left(\mathbf{x}_{I}\right)\right), \boldsymbol{u}\right\rangle \mathrm{R}^{2} \mathrm{~d}(\boldsymbol{\pi} \otimes \lambda) \\
& =\int \eta(t)\left\langle\left(\mathrm{D}_{x} \xi(x), 2 \xi(x)\right), \boldsymbol{u}_{I}\right\rangle \mathrm{d} \mu_{I} \\
& =\int_{0}^{1} \eta(t) \int_{\mathbb{R}^{d}}\left(\left\langle\mathrm{D}_{x} \xi(x), \boldsymbol{v}_{t}(x)\right\rangle+2 \xi(x) \hat{w}_{t}(x)\right) \mathrm{d} \mu_{t} \mathrm{~d} t .
\end{aligned}
$$

Setting $w_{t}=2 \hat{w}_{t}$ the continuity equation with reaction (8.65) holds.

The next result provides the opposite inequality, which will be deduced from the duality between the solutions of the generalized Hamilton-Jacobi equation and $\mathrm{HK}$ developed in Theorem 8.12.

Theorem 8.17 Let $\left(\mu_{t}\right)_{t \in[0,1]}$ be a continuous curve in $\mathcal{M}\left(\mathbb{R}^{d}\right)$ that solves the continuity equation with reaction (8.63) governed by the Borel vector field $(\boldsymbol{v}, w) \in L^{2}\left(\mathbb{R}^{d} \times[0,1], \mu_{I} ; \mathbb{R}^{d+1}\right)$ with $\mu_{I}$ given by (8.61). Then $\mu \in$ $\mathrm{AC}^{2}\left([0,1] ;\left(\mathcal{M}\left(\mathbb{R}^{d}\right), \mathrm{K}\right)\right)$ and

$$
\left|\mu_{t}^{\prime}\right|^{2} \leq \int_{\mathbb{R}^{d}}\left(\left|\boldsymbol{v}_{t}\right|^{2}+\frac{1}{4}\left|w_{t}\right|^{2}\right) \mathrm{d} \mu_{t} \text { for } \mathscr{L}^{1} \text {-a.e. } t \in(0,1) .
$$

Proof The simple scaling $\xi(t, x) \rightarrow(b-a) \xi(a+(b-a) t, x)$ transforms any subsolution of the Hamilton-Jacobi equation in $[0,1]$ to a subsolution of the same equation in $[a, b]$. Thus, Corollary 8.14 yields

$$
\begin{gathered}
\mathrm{H}^{2}\left(\mu_{0}, \mu_{1}\right)=2(b-a) \sup \left\{\int_{\mathbb{R}^{d}} \xi_{b} \mathrm{~d} \mu_{1}-\int_{\mathbb{R}^{d}} \xi_{a} \mathrm{~d} \mu_{0}: \xi \in \mathrm{C}_{\mathrm{c}}^{\infty}\left(\mathbb{R}^{d} \times[a, b]\right),\right. \\
\left.\partial_{t} \xi_{t}(x)+\frac{1}{2}\left|\mathrm{D}_{x} \xi_{t}(x)\right|^{2}+2 \xi_{t}^{2}(x) \leq 0 \text { in } \mathbb{R}^{d} \times(a, b)\right\} .
\end{gathered}
$$

Let $\xi \in \mathrm{C}_{\mathrm{c}}^{\infty}\left(\mathbb{R}^{d} \times[0,1]\right)$ be a subsolution to the Hamilton-Jacobi equation $\partial_{t} \xi+\frac{1}{2}|\mathrm{D} \xi|^{2}+2 \xi^{2} \leq 0$ in $\mathbb{R}^{d} \times[0,1]$. By a standard argument (see [2, Lem. 8.1.2]), the integrability (8.62), the weak continuity of $t \mapsto \mu_{t}$ and (8.64) yield

$$
\begin{aligned}
& 2 \int_{\mathbb{R}^{d}} \xi_{t_{1}} \mathrm{~d} \mu_{t_{1}}-2 \int_{\mathbb{R}^{d}} \xi_{t_{0}} \mathrm{~d} \mu_{t_{0}}=2 \int_{t_{0}}^{t_{1}} \int_{\mathbb{R}^{d}}\left(\partial_{t} \xi_{t}+\left\langle\mathrm{D}_{x} \xi_{t}, \boldsymbol{v}_{t}\right\rangle+\xi_{t} w_{t}\right) \mathrm{d} \mu_{t} \mathrm{~d} t \\
& \quad \leq 2 \int_{t_{0}}^{t_{1}} \int_{\mathbb{R}^{d}}\left(-\frac{1}{2}\left|\mathrm{D}_{x} \xi_{t}\right|^{2}-2 \xi_{t}^{2}+\left\langle\mathrm{D}_{x} \xi_{t}, \boldsymbol{v}_{t}\right\rangle+\xi_{t} w_{t}\right) \mathrm{d} \mu_{t} \mathrm{~d} t \\
& \quad \leq \int_{t_{0}}^{t_{1}} \int_{\mathbb{R}^{d}}\left(\left|\boldsymbol{v}_{t}\right|^{2}+\frac{1}{4}\left|w_{t}\right|^{2}\right) \mathrm{d} \mu_{t} \mathrm{~d} t
\end{aligned}
$$


Applying Corollary 8.14 and (8.70) we find

$$
\mathrm{H}^{2}\left(\mu_{t_{0}}, \mu_{t_{1}}\right) \leq\left(t_{1}-t_{0}\right) \int_{t_{0}}^{t_{1}} \int_{\mathbb{R}^{d}}\left(\left|\boldsymbol{v}_{t}\right|^{2}+\frac{1}{4}\left|w_{t}\right|^{2}\right) \mathrm{d} \mu_{t} \mathrm{~d} t
$$

for every $0 \leq t_{0}<t_{1} \leq 1$, which yields (8.69).

Combining Theorems 8.16 and 8.17 with Theorem 8.4 and the geodesic property of $\left(\mathcal{M}\left(\mathbb{R}^{d}\right), \mathrm{H}\right)$ we immediately have the desired dynamic representation.

Theorem 8.18 (Representation of $\mathrm{H}$ à la Benamou-Brenier) For every $\mu_{0}, \mu_{1} \in \mathcal{M}\left(\mathbb{R}^{d}\right)$ we have

$$
\begin{aligned}
\mathrm{HK}^{2}\left(\mu_{0}, \mu_{1}\right)= & \min \left\{\int_{0}^{1} \int_{\mathbb{R}^{d}}\left(\left|\boldsymbol{v}_{t}\right|^{2}+\frac{1}{4}\left|w_{t}\right|^{2}\right) \mathrm{d} \mu_{t} \mathrm{~d} t:\right. \\
& \mu \in \mathrm{C}\left([0,1] ; \mathcal{M}\left(\mathbb{R}^{d}\right)\right), \mu_{t=i}=\mu_{i}, \\
& \left.\partial_{t} \mu_{t}+\nabla \cdot\left(\boldsymbol{v}_{t} \mu_{t}\right)=w_{t} \mu_{t} \text { in } \mathscr{D}^{\prime}\left(\mathbb{R}^{d} \times(0,1)\right)\right\} .
\end{aligned}
$$

The Borel vector field $(\boldsymbol{v}, w)$ realizing the minimum in (8.71) is uniquely determined $\mu_{I}$-a.e. in $\mathbb{R}^{d} \times(0,1)$.

The discussion in [30] reveals however that there may be many geodesic curves, so in general $\mu_{I}$ is not unique. Indeed, the set of all geodesics connecting $\mu_{0}=a_{0} \delta_{x_{0}}$ and $\mu_{1}=a_{1} \delta_{x_{1}}$ with $a_{0}, a_{1}>0$ and $\left|x_{1}-x_{0}\right|=\pi / 2$ is infinite dimensional, see [30, Sect. 5.2].

Remark 8.19 (Inf-convolution of length distances) Here we want to explain why we may interpret the characterization (8.71) of $\mathrm{HK}$ as an infimal convolution (shortly inf-convolution) of the Kantorovich-Wasserstein distance $\mathbf{W}_{\mathrm{d}}$ and the Hellinger-Kakutani distance He.

Let us first recall that if $\|\cdot\|_{i}, i=1,2$, are Hilbert norms on a linear space $V$, the classical inf-convolution for convex functionals induces the infconvolution Hilbertian norm $\|\cdot\|_{\nabla}$ defined by

$$
\|v\|_{\nabla}^{2}:=\inf \left\{\left\|v_{1}\right\|_{1}^{2}+\left\|v_{2}\right\|_{2}^{2}: v=v_{1}+v_{2}\right\} .
$$

When a finite dimensional manifold $M$ is endowed with two Riemannian tensors $g_{1}$ and $g_{2}$, we can define the inf-convolution distance by computing the inf-convolution of the metric tensors in each tangent space. This leads to 
the formula

$$
\begin{aligned}
& \mathrm{d}_{\nabla}^{2}\left(x_{0}, x_{1}\right)=\inf \{\left\{\int _ { 0 } ^ { 1 } \left(\left|v_{1}(s)\right|_{\mathrm{g}_{1}(\mathrm{x}(s))}^{2}+\left|v_{2}(s)\right|_{\mathrm{g}_{2}(\mathrm{x}(s))}^{2} \mathrm{~d} s:\right.\right. \\
& \mathrm{x} \in \mathrm{C}^{1}([0,1] ; M), \mathrm{x}(i)=x_{i}, \\
&\left.\dot{\mathrm{x}}(s)=v_{1}(s)+v_{2}(s) \text { in } T_{\mathrm{x}(s)}(M) \text { for a.e. } s \in(0,1)\right\} .
\end{aligned}
$$

By optimizing the decomposition $\dot{x}=v_{1}+v_{2}$ we easily find that the infconvolution distance is generated by the metric tensor $g_{\nabla}$ whose dual $g_{\nabla}^{*}$ is given by $g_{\nabla}^{*}=g_{1}^{*}+g_{2}^{*}$. This formula reflects the fact that the Legendre transform of an inf-convolution is the sum of the two Legendre transforms of the convoluted functionals. One can think that (8.71) exhibits a non-smooth, infinite dimensional example sharing the same structure. For another infinitedimensional application we refer to [8, Eq. (16)].

When $\mathrm{d}_{i}, i=1,2$, are length metrics on a given set $Z$, a purely metric infconvolution $\mathrm{d}_{\nabla}=\mathrm{d}_{1} \nabla \mathrm{d}_{2}$ respecting the local Hilbert-space structure reads

$$
\begin{aligned}
\mathrm{d}_{\nabla}^{2}\left(z_{1}, z_{2}\right):=\liminf _{N \rightarrow \infty} \inf \left\{N \sum_{i=1}^{N}\left(\mathrm{~d}_{1}^{2}\left(x_{i-1}, y_{i}\right)+\mathrm{d}_{2}^{2}\left(y_{i}, x_{i}\right)\right):\right. \\
\left.x_{i}, y_{i} \in Z, x_{0}=z_{1}, y_{N}=z_{2}\right\} .
\end{aligned}
$$

One can expect that this inf-convolution applied to $W_{d}$ and He exactly generates $\mathrm{H}$, namely $\mathrm{H}=\mathrm{W}_{\mathrm{d}} \underset{\mathrm{inf}}{\nabla} \mathrm{He}$.

\subsection{Geodesics in $\mathcal{M}\left(\mathbb{R}^{d}\right)$}

As in the case of the Kantorovich-Wasserstein distance, one may expect that geodesics $\left(\mu_{t}\right)_{t \in[0,1]}$ in $\left(\mathcal{M}\left(\mathbb{R}^{d}\right), \mathrm{K}\right)$ can be characterized by the system (cf. [30, Sect. 5])

$$
\partial_{t} \mu_{t}+\nabla \cdot\left(\mu_{t} \mathrm{D}_{x} \xi_{t}\right)=4 \xi_{t} \mu_{t}, \quad \partial_{t} \xi_{t}+\frac{1}{2}\left|\mathrm{D}_{x} \xi_{t}\right|^{2}+2 \xi_{t}^{2}=0
$$

In order to give a precise meaning to (8.72) we first have to select an appropriate regularity for $\xi_{t}$. On the one hand we cannot expect $\mathrm{C}^{1}$ smoothness for solutions of the Hamilton-Jacobi equation (8.72) (in contrast with subsolutions, that can be regularized as in Corollary 8.14) and on the other hand the $\mathscr{L}^{d}$ a.e. differentiability of Lipschitz functions guaranteed by Rademacher's theorem is not sufficient, if we want to consider arbitrary measures $\mu_{t}$ that could be singular with respect $\mathscr{L}^{d}$. 
A convenient choice for our aims is provided by locally Lipschitz functions which are strictly differentiable at $\mu_{I}$-a.e. points, where $\mu_{I}$ has been defined by (8.61). A function $f: \mathbb{R}^{d} \rightarrow \mathbb{R}$ is strictly differentiable at $x \in \mathbb{R}^{d}$ if there exists $\mathrm{D} f(x) \in\left(\mathbb{R}^{d}\right)^{*}$ such that

$$
\lim _{\substack{x^{\prime}, x^{\prime \prime} \rightarrow x \\ x^{\prime} \neq x^{\prime \prime}}} \frac{f\left(x^{\prime}\right)-f\left(x^{\prime \prime}\right)-\mathrm{D} f(x)\left(x^{\prime}-x^{\prime \prime}\right)}{\left|x^{\prime}-x^{\prime \prime}\right|}=0 .
$$

According to [15, Prop. 2.2.4] a locally Lipschitz function $f$ is strictly differentiable at $x$ if and only if the Clarke subgradient [15, Sect. 2.1] of $f$ at $x$ reduces to the singleton $\{\mathrm{D} f(x)\}$. In particular, denoting by $\boldsymbol{D} \subset \mathbb{R}^{d}$ the set where $f$ is differentiable and denoting by $\kappa_{\varepsilon}$ a smooth convolution kernel as in (8.58), Rademacher's theorem and [15, Thm. 2.5.1] yield

$$
\lim _{\substack{x^{\prime} \rightarrow x \\ x^{\prime} \in \boldsymbol{D}}} \mathrm{D} f\left(x^{\prime}\right)=\mathrm{D} f(x), \quad \lim _{\varepsilon \downarrow 0} \mathrm{D}\left(f * \kappa_{\varepsilon}\right)(x)=\mathrm{D} f(x) \text { for all } x \in \boldsymbol{D} .
$$

In the proofs we will also need to deal with pointwise representatives of the time derivative of a locally Lipschitz function $\xi: \mathbb{R}^{d} \times(0,1) \rightarrow \mathbb{R}:$ if $D\left(\partial_{t} \xi\right)$ will denote the set (of full $\mathscr{L}^{d+1}$ measure) where $\xi$ is differentiable w.r.t. time and $\widetilde{\partial_{t} \xi}$ the extension of $\partial_{t} \xi$ to 0 outside $D\left(\partial_{t} \xi\right)$, we set

$$
\left(\partial_{t} \xi_{t}\right)_{-}(x):=\liminf _{\varepsilon \rightarrow 0}\left(\widetilde{\partial_{t} \xi_{t}} * \kappa_{\varepsilon}\right)(x), \quad\left(\partial_{t} \xi_{t}\right)^{+}(x):=\limsup _{\varepsilon \rightarrow 0}\left(\widetilde{\partial_{t} \xi_{t}} * \kappa_{\varepsilon}\right)(x) .
$$

It is not difficult to check that such functions are Borel; even if they depend on the specific choice of $\kappa_{\varepsilon}$, they will still be sufficient for our aims (a more robust definition would require the use of approximate limits).

We are now ready to characterize the set of all geodesic curves by giving a precise meaning to (8.72). The proof that the conditions (i)-(iv) below are sufficient for geodesic follows directly with the subsequent Lemma 8.21, whereas the proof of necessity is more involved and relies on the existence of optimal potentials $\psi_{1}$ for LET $=\mathrm{HK}^{2}$ in Theorem 6.3(d), on the characterization of subsolutions of the generalized Hamilton-Jacobi equation in Theorem 8.11, and on the characterization of curves $t \mapsto \mu_{t}$ in $\mathrm{AC}^{2}\left([0,1] ;\left(\mathcal{M}\left(\mathbb{R}^{d}\right), \mathrm{HK}\right)\right)$.

Theorem 8.20 Let $\mu \in \mathrm{C}^{0}\left([0,1] ; \mathcal{M}\left(\mathbb{R}^{d}\right)\right)$ be a weakly continuous curve. If there exists a map $\xi \in \operatorname{Lip}_{\text {loc }}\left((0,1) ; \mathrm{C}_{b}\left(\mathbb{R}^{d}\right)\right)$ such that

(i) $\xi_{t} \in \operatorname{Lip}_{b}\left(\mathbb{R}^{d}\right)$ for every $t \in(0,1)$ with $t \mapsto \operatorname{Lip}\left(\xi_{t}, \mathbb{R}^{d}\right)$ locally bounded in $(0,1)$ (equivalently, the map $(x, t) \mapsto \xi_{t}(x)$ is bounded and Lipschitz in $\mathbb{R}^{d} \times[a, b]$ for every compact subinterval $\left.[a, b] \subset(0,1)\right)$,

(ii) $\xi$ is strictly differentiable w.r.t. $x$ at $\mu_{I}$-a.e. $(x, t) \in \mathbb{R}^{d} \times(0,1)$, 
(iii) $\xi$ satisfies

$$
\partial_{t} \xi_{t}+\frac{1}{2}\left|\mathrm{D}_{x} \xi_{t}(x)\right|^{2}+2 \xi_{t}^{2}(x)=0 \quad \mathscr{L}^{d+1} \text {-a.e. in } \mathbb{R}^{d} \times(0,1)
$$

(iv) and the curve $\left(\mu_{t}\right)_{t \in[0,1]}$ solves the continuity equation with reaction with the vector field $\left(\mathrm{D}_{x} \xi, 4 \xi\right)$ in every compact subinterval of $(0,1)$, i.e.

$$
\partial_{t} \mu_{t}+\nabla \cdot\left(\mu_{t} \mathrm{D}_{x} \xi_{t}\right)=4 \xi_{t} \mu_{t} \quad \text { in } \mathscr{D}^{\prime}\left(\mathbb{R}^{d} \times(0,1)\right)
$$

then $\mu$ is a geodesic w.r.t. the $\mathrm{HK}$ distance. Conversely, if $\mu$ is a geodesic then it is possible to find $\xi \in \operatorname{Lip}_{\mathrm{loc}}\left((0,1) ; \mathrm{C}_{b}\left(\mathbb{R}^{d}\right)\right)$ that satisfies the properties (i)-(iv) above, is right differentiable w.r.t. $t$ in $\mathbb{R}^{d} \times(0,1)$, and fulfills $(8.50 \mathrm{~b})$ everywhere in $\mathbb{R}^{d} \times(0,1)$.

Notice that (8.76) seems the weakest natural formulation of the HamiltonJacobi equation, in view of Rademacher's Theorem. The assumption of strict differentiability of $\xi$ at $\mu_{I}$-a.e. point provides an admissible vector field $\mathrm{D}_{X} \xi$ for (8.77).

Proof The proof splits into a sufficiency and a necessity part, the latter having several steps.

Sufficiency. Let us suppose that $\mu, \xi$ satisfy conditions $(i), \ldots,(i v)$.

Since $D\left(\partial_{t} \xi\right)$ has full $\mathscr{L}^{d+1}$-measure in $\mathbb{R}^{d} \times(0,1)$, Fubini's Theorem shows that $N:=\left\{t \in(0,1): \mathscr{L}^{d}\left(\left\{x \in \mathbb{R}^{d}:(x, t) \notin D\left(\partial_{t} \xi\right)\right\}\right)>0\right\}$ is $\mathscr{L}^{1}$-negligible. By (8.76) we get

$$
\left(\partial_{t} \xi\right)_{-}(x)=-\limsup _{\varepsilon \downarrow 0}\left(\left(\frac{1}{2}\left|\mathrm{D}_{x} \xi_{t}\right|^{2}+2 \xi_{t}^{2}\right) * \kappa_{\varepsilon}\right)(x) \geq-\frac{1}{2}\left|\mathrm{D} \xi_{t}\right|_{a}^{2}(x)-2 \xi_{t}^{2}(x)
$$

for every $x \in \mathbb{R}^{d}$ and $t \in(0,1) \backslash N$.

We apply Lemma 8.21 below with $v=\mathrm{D}_{x} \xi$ and $w=4 \xi$ : observing that $\left|\mathrm{D} \xi_{t}\right|_{a}(x)=\left|\mathrm{D}_{x} \xi_{t}(x)\right|$ at every point $x$ of strict differentiability of $\xi_{t}$, we get, for all $0<a<b<1$,

$$
\begin{aligned}
& 2 \int_{\mathbb{R}^{d}} \xi_{b} \mathrm{~d} \mu_{b}-2 \int_{\mathbb{R}^{d}} \xi_{a} \mathrm{~d} \mu_{a} \geq 2 \int_{\mathbb{R}^{d} \times(a, b)}\left(\left(\partial_{t} \xi\right)_{-}+\left|\mathrm{D}_{x} \xi_{t}(x)\right|^{2}+4 \xi_{t}^{2}(x)\right) \mathrm{d} \mu_{I} \\
& \stackrel{(8.78)}{=} 2 \int_{\mathbb{R}^{d} \times(a, b)}\left(\frac{1}{2}\left|\mathrm{D}_{x} \xi_{t}(x)\right|^{2}+2 \xi_{t}^{2}(x)\right) \mathrm{d} \mu_{I} \\
& \stackrel{(8.69)}{\geq} \int_{a}^{b}\left|\mu_{t}^{\prime}\right|^{2} \mathrm{~d} t \geq \frac{1}{b-a} \mathrm{H}^{2}\left(\mu_{a}, \mu_{b}\right) .
\end{aligned}
$$


On the other hand, since $\mathbb{R}^{d}$ is a length space, Theorem 8.12 yields

$$
\frac{1}{b-a} \mathrm{H}^{2}\left(\mu_{a}, \mu_{b}\right) \geq 2 \int_{\mathbb{R}^{d}} \xi_{b} \mathrm{~d} \mu_{b}-2 \int_{\mathbb{R}^{d}} \xi_{a} \mathrm{~d} \mu_{a},
$$

so that all the above inequalities are in fact identities and, hence,

$$
\mathrm{HK}\left(\mu_{a}, \mu_{b}\right)=(b-a)\left|\mu_{t}^{\prime}\right| \mathscr{L}^{1} \text {-a.e. in }[a, b] .
$$

This shows that $\mu$ is a geodesic. Passing to the limit as $a \downarrow 0$ and $b \uparrow 1$ we conclude the proof of the first part of the Theorem.

Necessity. Let $\left(\mu_{t}\right)_{t \in[0,1]}$ be a $\mathrm{K}$-geodesic in $\mathcal{M}\left(\mathbb{R}^{d}\right)$ connecting $\mu_{0}$ to $\mu_{1}$; applying Theorem 8.16 we can find a Borel vector field $(\boldsymbol{v}, w) \in \mathrm{L}^{2}\left(\mathbb{R}^{d} \times\right.$ $\left.(0,1), \mu_{I} ; \mathbb{R}^{d+1}\right)$ such that (8.63) and (8.66) hold. We also consider an optimal plan $\boldsymbol{\gamma} \in \mathrm{Opt}_{\text {ETT }}\left(\mu_{1}, \mu_{2}\right)$.

Let $\psi_{1}, \psi_{2}: \mathbb{R}^{d} \rightarrow[-\infty, 1]$ be a pair of optimal potentials given by Theorem $6.3 \mathrm{~d})$ and let us set $\xi:=-\frac{1}{2} \psi_{1}$ and $\xi_{t}:=\mathscr{P}_{t} \xi$ for $t \in(0,1)$. Even if we are considering more general initial data $\xi \in \mathrm{B}\left(\mathbb{R}^{d} ;[-1 / 2, \infty]\right)$ in (8.48), it is not difficult to check that the same statement of Theorem 8.11 holds in every subinterval $[a, b]$ with $0<a<b<1$ and

$$
\lim _{t \downarrow 0} \mathscr{P}_{t} \xi(x)=\sup _{t>0} \mathscr{P}_{t} \xi(x)=\xi_{*}(x), \quad \text { where } \quad \xi_{*}(x):=\lim _{r \downarrow 0} \inf _{x^{\prime} \in B_{r}(x)} \xi\left(x^{\prime}\right)
$$

is the lower semicontinuous envelope of $\xi$. Moreover, setting

$$
\xi_{1}(x)=\mathscr{P}_{1} \xi(x):=\lim _{t \uparrow 1} \xi_{t}(x)=\inf _{0<t<1} \xi_{t}(x),
$$

the function $\xi_{1}$ is upper semicontinuous with values in $[-\infty, 1 / 2]$ and the optimality properties stated in Theorem $6.3 \mathrm{~d}$ ) yield

$$
\frac{1}{2} \psi_{2} \leq \xi_{1} \quad \text { in } \mathbb{R}^{d}, \quad \frac{1}{2} \psi_{2}=\xi_{1} \quad \mu_{1} \text {-a.e. in } \mathbb{R}^{d} .
$$

By introducing the semigroup $\overline{\mathscr{P}}_{t} \xi:=-\mathscr{P}_{t}(-\xi)$ and reversing time, we can define

$$
\bar{\xi}_{t}:=\overline{\mathscr{P}}_{1-t}\left(\frac{1}{2} \psi_{2}\right) \text {. }
$$

By using the link with the Hopf-Lax semigroup in $\mathfrak{C}$ given by Theorem 8.11, the optimality of $\left(\psi_{1}, \psi_{2}\right)$, and arguing as in [50, Thm. 7.36] it is not difficult to check that

$$
\bar{\xi}_{t} \leq \xi_{t} \quad \text { in } \mathbb{R}^{d}, \quad \bar{\xi}_{0}=\xi_{0}=-\frac{1}{2} \psi_{1} \quad \mu_{0} \text {-a.e. in } \mathbb{R}^{d} .
$$


Notice that the function $x \mapsto-\cos ^{2}\left(\left|x-x^{\prime}\right| \wedge \pi / 2\right)$ has bounded first and second derivatives, so it is semiconcave. It follows that the map $x \mapsto \xi_{t}(x)$ is semiconcave for every $t \in(0,1)$ and $x \mapsto \bar{\xi}_{t}(x)$ is semiconvex.

Since $t \mapsto \int \xi_{t} \mathrm{~d} \mu_{t}$ and $t \mapsto \int \bar{\xi}_{t} \mathrm{~d} \mu_{t}$ are absolutely continuous in $(0,1)$, Theorem 8.12(i) yields

$$
\frac{\mathrm{d}}{\mathrm{d} t} \int \xi_{t} \mathrm{~d} \mu_{t} \leq \frac{1}{2}\left|\mu_{t}^{\prime}\right|^{2}=\frac{1}{2} \mathrm{H}^{2}\left(\mu_{0}, \mu_{1}\right),
$$

so that

$$
\int \xi_{b} \mathrm{~d} \mu_{b}-\int \xi_{a} \mathrm{~d} \mu_{a} \leq \frac{b-a}{2} \mathrm{H}^{2}\left(\mu_{0}, \mu_{1}\right)
$$

Passing to the limit first as $a \downarrow 0$ and then as $b \uparrow 1$ by monotone convergence (notice that $\xi_{t} \leq 1 / 2$ ) and using optimality once again, we obtain

$$
\begin{aligned}
\mathrm{H}^{2}\left(\mu_{0}, \mu_{1}\right) & =\int \psi_{1} \mathrm{~d} \mu_{0}+\int \psi_{2} \mathrm{~d} \mu_{1}=2 \int \xi_{1} \mathrm{~d} \mu_{1}-2 \int \xi_{0} \mathrm{~d} \mu_{0} \\
& =\lim _{a \downarrow 0, b \uparrow 1} 2\left(\int \xi_{b} \mathrm{~d} \mu_{b}-\int \xi_{a} \mathrm{~d} \mu_{a}\right) .
\end{aligned}
$$

By (8.84) it follows that

$$
\frac{\mathrm{d}}{\mathrm{d} t} \int \xi_{t} \mathrm{~d} \mu_{t}=\frac{1}{2}\left|\mu_{t}^{\prime}\right|^{2}=\frac{1}{2} \mathrm{H}^{2}\left(\mu_{0}, \mu_{1}\right) \quad \text { in }(0,1)
$$

Reversing time, the analogous argument yields

$$
\frac{\mathrm{d}}{\mathrm{d} t} \int \bar{\xi}_{t} \mathrm{~d} \mu_{t}=\frac{1}{2}\left|\mu_{t}^{\prime}\right|^{2}=\frac{1}{2} \mathrm{~K}^{2}\left(\mu_{0}, \mu_{1}\right) \text { in }(0,1) .
$$

Hence, we have proved that the maps $t \mapsto \int \xi_{t} \mathrm{~d} \mu_{t}$ and $t \mapsto \int \bar{\xi}_{t} \mathrm{~d} \mu_{t}$ are affine in $[0,1]$ and coincide at $t=0$ and $t=1$, which implies that

$$
\int \xi_{t} \mathrm{~d} \mu_{t}=\int \bar{\xi}_{t} \mathrm{~d} \mu_{t} \quad \text { for every } t \in[0,1] .
$$

Recalling (8.83), we deduce that the complement of the set $Z_{t}:=\left\{x \in \mathbb{R}^{d}\right.$ : $\left.\xi_{t}(x)=\bar{\xi}_{t}(x)\right\}$ is $\mu_{t}$-negligible. Since $\xi_{t}$ is Lipschitz and semiconcave (thus everywhere superdifferentiable) for $t \in(0,1)$ and since $\bar{\xi}_{t}$ is Lipschitz and semiconvex (thus everywhere subdifferentiable), we conclude that $\xi_{t}$ is strictly differentiable in $Z_{t}$, and thus it satisfies conditions (i) and (ii). 
Since (iii) is guaranteed by Theorem 8.11 ( $\mathbb{R}^{d}$ is a length space), it remains to check (8.77). We apply the following Lemma 8.21 by observing that [3, Prop. 3.2,3.3] and Theorem 8.11 yield

$\limsup _{x^{\prime} \rightarrow x} \partial_{t}^{+} \xi_{t}\left(x^{\prime}\right) \leq \limsup _{x^{\prime} \rightarrow x} \partial_{t}^{-} \xi_{t}\left(x^{\prime}\right) \leq \partial_{t}^{-} \xi_{t}(x), \liminf _{x^{\prime} \rightarrow x} \partial_{t}^{+} \xi_{t}\left(x^{\prime}\right) \geq \partial_{t}^{+} \xi_{t}(x) ;$ since $\partial_{t}^{-} \xi_{t}(x)=\partial_{t}^{+} \xi_{t}(x) \mu_{I}$-a.e. we get $\left(\partial_{t} \xi\right)^{+}=\left(\partial_{t} \xi\right)_{-}=\partial_{t}^{+} \xi \mu_{I}$-a.e. and therefore (8.89) holds with equality.

Recalling that $\left|\mathrm{D} \xi_{t}\right|_{a}^{2}(x)=\left|\mathrm{D}_{x} \xi_{t}(x)\right|^{2}$ at every point of $Z_{t}$, for every $0<$ $a<b<1$ we have

$$
\begin{aligned}
& \frac{b-a}{2} \mathrm{HK}^{2}\left(\mu_{0}, \mu_{1}\right)=\int_{\mathbb{R}^{d}} \xi_{b} \mathrm{~d} \mu_{b}-\int_{\mathbb{R}^{d}} \xi_{a} \mathrm{~d} \mu_{a} \\
& \stackrel{(8.89)}{=} \int_{\mathbb{R}^{d} \times(a, b)}\left(\partial_{t}^{+} \xi+\mathrm{D}_{x} \xi \boldsymbol{v}+\xi w\right) \mathrm{d} \mu_{I} \\
& =\int_{\mathbb{R}^{d} \times(a, b)}\left(-\frac{1}{2}\left|\mathrm{D}_{x} \xi_{t}\right|^{2}-2 \xi_{t}^{2}+\mathrm{D}_{x} \xi \boldsymbol{v}+\xi w\right) \mathrm{d} \mu_{I} \\
& =\int_{\mathbb{R}^{d} \times(a, b)}\left(-\frac{1}{2}\left|\mathrm{D}_{x} \xi_{t}-\boldsymbol{v}\right|^{2}-2\left(\xi_{t}-\frac{1}{4} w\right)^{2}+\frac{1}{2}|\boldsymbol{v}|^{2}+\frac{1}{8} w^{2}\right) \mathrm{d} \mu_{I} \\
& \stackrel{(8.66)}{\leq}-\int_{\mathbb{R}^{d} \times(a, b)}\left(\frac{1}{2}\left|\mathrm{D}_{x} \xi_{t}-\boldsymbol{v}\right|^{2}+2\left(\xi_{t}-\frac{1}{4} w\right)^{2}\right) \mathrm{d} \mu_{I}+\frac{1}{2} \int_{a}^{b}\left|\mu_{t}^{\prime}\right|^{2} \mathrm{~d} t
\end{aligned}
$$

We deduce that $\boldsymbol{v}=\mathrm{D}_{x} \xi$ and $w=4 \xi$ holds $\mu_{I}$-a.e.

The following lemma provides the "integration by parts" formulas that where used in the sufficiency and necessity part of the previous proof of Theorem 8.20. It is established by a suitable temporal and spatial smoothing, involving a smooth kernel $\kappa_{\varepsilon}$ as in (8.58).

Lemma 8.21 Let $\mu \in \operatorname{AC}_{\mathrm{loc}}^{2}\left((0,1) ;\left(\mathcal{M}\left(\mathbb{R}^{d}\right), \mathrm{K}\right)\right)$ satisfy the continuity equation with reaction (8.63) governed by the field $(\boldsymbol{v}, w) \in L^{2}\left(\mathbb{R}^{d} \times\right.$ $\left.(a, b), \mu_{I}\right)$ for every $[a, b] \subset(0,1)$. If $\xi \in \operatorname{Lip}_{\text {loc }}\left((0,1) ; \mathrm{C}_{b}\left(\mathbb{R}^{d}\right)\right)$ satisfies conditions $(i, i i)$ of Theorem 8.20, then for all $0<a \leq b<1$ we have

$$
\begin{aligned}
& \int_{\mathbb{R}^{d} \times(a, b)}\left(\left(\partial_{t} \xi\right)^{+}+\mathrm{D}_{x} \xi \boldsymbol{v}+\xi w\right) \mathrm{d} \mu_{I} \geq \int_{\mathbb{R}^{d}} \xi_{b} \mathrm{~d} \mu_{b}-\int_{\mathbb{R}^{d}} \xi_{a} \mathrm{~d} \mu_{a} \\
& \quad \geq \int_{\mathbb{R}^{d} \times(a, b)}\left(\left(\partial_{t} \xi\right)_{-}+\mathrm{D}_{x} \xi \boldsymbol{v}+\xi w\right) \mathrm{d} \mu_{I},
\end{aligned}
$$

where $\left(\partial_{t} \xi\right)^{+},\left(\partial_{t} \xi\right)_{-}$are defined in terms of a space convolution kernel $\kappa_{\varepsilon}$ as in (8.75). 
Proof We fix a compact subinterval $[a, b] \subset(0,1), b^{\prime} \in(b, 1)$, and set $M:=$ $\max _{t \in\left[a, b^{\prime}\right]} \mu_{t}\left(\mathbb{R}^{d}\right)$ and $L:=\operatorname{Lip}\left(\xi ; \mathbb{R}^{d} \times\left[a, b^{\prime}\right]\right)+\sup _{\mathbb{R}^{d} \times\left[a, b^{\prime}\right]}|\xi|$.

We regularize $\xi$ by space convolution as in (8.58) by setting $\xi^{\varepsilon}:=\xi * \kappa_{\varepsilon}$ and perform a further regularization in time, viz.

$$
\xi_{t}^{\varepsilon, \tau}(x):=\frac{1}{\tau} \int_{0}^{\tau} \xi_{t+r}^{\varepsilon}(x) \mathrm{d} r, \quad 0<\tau<b^{\prime}-b .
$$

Since $\xi^{\varepsilon, \tau} \in \mathrm{C}_{b}^{1}\left(\mathbb{R}^{d} \times[a, b]\right)$ and $\mu$ is a weakly continuous solution to (8.63), we can argue as in [2, Lem. 8.1.2] and obtain, for every $\varepsilon>0$ and $\tau \in$ $\left(0, b^{\prime}-b\right)$, the identity

$$
\int_{\mathbb{R}^{d}} \xi_{b}^{\varepsilon, \tau} \mathrm{d} \mu_{b}-\int_{\mathbb{R}^{d}} \xi_{a}^{\varepsilon, \tau} \mathrm{d} \mu_{a}=\int_{\mathbb{R}^{d} \times(a, b)}\left(\partial_{t} \xi^{\varepsilon, \tau}+\mathrm{D}_{x} \xi^{\varepsilon, \tau} \boldsymbol{v}+\xi^{\varepsilon, \tau} w\right) \mathrm{d} \mu_{I} .
$$

We first pass to the limit as $\tau \downarrow 0$, observing that $\xi^{\varepsilon, \tau} \rightarrow \xi^{\varepsilon}$ uniformly because $\xi^{\varepsilon}$ is bounded and Lipschitz. Similarly, since $\mathrm{D} \xi^{\varepsilon, \tau}=\left(\mathrm{D} \xi^{\varepsilon}\right)^{\tau}$ and $\mathrm{D} \xi^{\varepsilon}$ is bounded and Lipschitz, we have $\mathrm{D} \xi^{\varepsilon, \tau} \rightarrow \mathrm{D} \xi^{\varepsilon}$ uniformly. Finally, using

$\partial_{t} \xi_{t}^{\varepsilon, \tau}(x)=\frac{1}{\tau}\left(\xi_{t+\tau}^{\varepsilon}(x)-\xi_{t}^{\varepsilon}(x)\right)=\int_{\mathbb{R}^{d}} \frac{1}{\tau}\left(\xi_{t+\tau}^{\varepsilon}\left(x^{\prime}\right)-\xi_{t}^{\varepsilon}\left(x^{\prime}\right)\right) \kappa_{\varepsilon}\left(x-x^{\prime}\right) \mathrm{d} x^{\prime}$,

and the fact that $N:=\left\{t \in(0,1): \mathscr{L}^{d}\left(\left\{x \in \mathbb{R}^{d}:(x, t) \notin D\left(\partial_{t} \xi\right)\right\}\right)>0\right\}$ is $\mathscr{L}^{1}$-negligible by the theorems of Rademacher and Fubini, an application of Lebesgue's Dominated Convergence Theorem yields

$\lim _{\tau \downarrow 0} \partial_{t} \xi_{t}^{\varepsilon, \tau}(x)=\partial_{t} \xi_{t}^{\varepsilon}(x)=\left(\left(\partial_{t} \xi\right) * \kappa_{\varepsilon}\right)(x) \quad$ for every $x \in \mathbb{R}^{d}, \quad t \in(a, b) \backslash N$.

Since $\mathbb{R}^{d} \times N$ is also $\mu_{I}$-negligible, a further application of Lebesgue's Dominated Convergence Theorem yields

$$
\int_{\mathbb{R}^{d}} \xi_{b}^{\varepsilon} \mathrm{d} \mu_{b}-\int_{\mathbb{R}^{d}} \xi_{a}^{\varepsilon} \mathrm{d} \mu_{a}=\int_{\mathbb{R}^{d} \times(a, b)}\left(\partial_{t} \xi^{\varepsilon}+\mathrm{D}_{x} \xi^{\varepsilon} \boldsymbol{v}+\xi^{\varepsilon} w\right) \mathrm{d} \mu_{I}
$$

Now, (8.89) will be deduced by passing to the limit $\varepsilon \downarrow 0$ in (8.93) as follows. We observe that $\xi^{\varepsilon}$ converges uniformly to $\xi$ because $\xi$ is bounded and Lipschitz. Moreover, since $\lim _{\varepsilon \downarrow 0} \mathrm{D}_{x} \xi_{t}^{\varepsilon}(x)=\mathrm{D}_{x} \xi_{t}(x)$ at every point $x \in \mathbb{R}^{d}$ where $\xi_{t}$ is strictly differentiable, we obtain

$$
\begin{gathered}
\left|\mathrm{D}_{x} \xi^{\varepsilon} \boldsymbol{v}\right| \leq L|\boldsymbol{v}| \in \mathrm{L}^{1}\left(\mathbb{R}^{d} \times(a, b) ; \mu_{I}\right) \text { and } \\
\lim _{\varepsilon \downarrow 0} \mathrm{D}_{x} \xi^{\varepsilon}=\mathrm{D}_{x} \xi \quad \mu_{I} \text {-a.e. in } \mathbb{R}^{d} \times[a, b],
\end{gathered}
$$


so that

$$
\begin{aligned}
\lim _{\varepsilon \downarrow 0} \int_{\mathbb{R}^{d}} \xi_{a, b}^{\varepsilon} \mathrm{d} \mu_{a, b} & =\int_{\mathbb{R}^{d}} \xi_{a, b} \mathrm{~d} \mu_{a, b}, \\
\int_{\mathbb{R}^{d} \times(a, b)}\left(\mathrm{D}_{x} \xi^{\varepsilon} \boldsymbol{v}+\xi^{\varepsilon} w\right) \mathrm{d} \mu_{I} & =\int_{\mathbb{R}^{d} \times(a, b)}\left(\mathrm{D}_{x} \xi \boldsymbol{v}+\xi w\right) \mathrm{d} \mu_{I} .
\end{aligned}
$$

Finally, since $\partial_{t} \xi_{t}^{\varepsilon}$ is also uniformly bounded, Fatou's Lemma yields

$$
\begin{aligned}
& \underset{\varepsilon \downarrow 0}{\limsup } \int_{\mathbb{R}^{d} \times(a, b)} \partial_{t} \xi_{t}^{\varepsilon} \mathrm{d} \mu_{I} \leq \int_{\mathbb{R}^{d} \times(a, b)}\left(\partial_{t} \xi_{t}\right)^{+} \mathrm{d} \mu_{I}, \\
& \liminf _{\varepsilon \downarrow 0} \int_{\mathbb{R}^{d} \times(a, b)} \partial_{t} \xi_{t}^{\varepsilon} \mathrm{d} \mu_{I} \geq \int_{\mathbb{R}^{d} \times(a, b)}\left(\partial_{t} \xi_{t}\right)_{-} \mathrm{d} \mu_{I} .
\end{aligned}
$$

Thus, (8.89) follows from (8.93).

\subsection{Contraction properties: convolution and Heat equation in $\operatorname{RCD}(0, \infty)$ metric-measure spaces}

We conclude this paper with a few applications concerning contraction properties of the $\mathrm{HK}$ distance. The first one concerns the behavior with respect 1-Lipschitz maps.

Lemma 8.22 Let $\left(X, \mathrm{~d}_{X}\right),\left(Y, \mathrm{~d}_{Y}\right)$ be separable metric spaces and let $f$ : $X \rightarrow Y$ be a 1-Lipschitz map. Then $f_{\sharp}: \mathcal{M}(X) \rightarrow \mathcal{M}(Y)$ is 1-Lipschitz w.r.t. $\mathrm{H}$ :

$$
\mathrm{H}\left(f_{\sharp} \mu_{1}, f_{\sharp} \mu_{2}\right) \leq \mathrm{H}\left(\mu_{1}, \mu_{2}\right) .
$$

Proof It is sufficient to observe that the map $\mathfrak{f}: \mathfrak{C}_{X} \mapsto \mathfrak{C}_{Y}$ defined by $\mathfrak{f}([x, r]):=[f(x), r]$ satisfies $\mathrm{d}_{\mathfrak{C}_{Y}}\left(\mathfrak{f}\left(\left[x_{1}, r_{1}\right]\right), \mathfrak{f}\left(\left[x_{2}, r_{2}\right]\right)\right) \leq \mathrm{d}_{\mathfrak{C}_{X}}\left(\left[x_{1}, r_{1}\right]\right.$, $\left.\left[x_{2}, r_{2}\right]\right)$ for every $\left[x_{i}, r_{i}\right] \in \mathfrak{C}_{X}$. Thus $\mathfrak{f}_{\sharp}$ is a contraction from $\left(\mathcal{P}_{2}\left(\mathfrak{C}_{X}\right), \mathbf{W}_{\mathfrak{d}_{\mathfrak{C}_{X}}}\right)$ to $\left(\mathcal{P}_{2}\left(\mathfrak{C}_{Y}\right), \mathrm{W}_{\mathfrak{d}_{\mathfrak{C}_{Y}}}\right)$, and hence $f_{\sharp}$ satisfies $(8.94)$.

A second application concerns convolutions in $\mathbb{R}^{d}$.

Theorem 8.23 Let $X=\mathbb{R}^{d}$ with the Euclidean distance and let $v \in \mathcal{M}\left(\mathbb{R}^{d}\right)$. Then the map $\mu \mapsto \mu * v$ is contractive w.r.t. $\mathrm{H}$ if $v\left(\mathbb{R}^{d}\right)=1$ and, more generally, 


$$
\mathrm{H}^{2}\left(\mu_{1} * v, \mu_{2} * v\right) \leq v\left(\mathbb{R}^{d}\right) \mathrm{H}^{2}\left(\mu_{1}, \mu_{2}\right) \text { for } \mu_{1}, \mu_{2} \in \mathcal{M}\left(\mathbb{R}^{d}\right) .
$$

Proof The previous lemma shows that $\mathrm{HK}$ is invariant by isometries, in particular translations in $\mathbb{R}^{d}$, so that

$$
\mathrm{K}\left(\mu_{1} * \delta_{x}, \mu_{2} * \delta_{x}\right)=\mathrm{K}\left(\mu_{1}, \mu_{2}\right) \text { for every } \mu_{1}, \mu_{2} \in \mathcal{M}\left(\mathbb{R}^{d}\right), x \in \mathbb{R}^{d} .
$$

By the subadditivity property (7.31), it follows that if $v=\sum_{k} a_{k} \delta_{x_{k}}$ for some $a_{k} \geq 0$, then

$$
\begin{aligned}
\mathrm{H}^{2}\left(\mu_{1} * v, \mu_{2} * v\right) & =\mathrm{H}^{2}\left(\sum_{k} a_{k} \mu_{1} * \delta_{x_{k}}, \sum_{k} a_{k} \mu_{2} * \delta_{x_{k}}\right) \\
& \leq \sum_{k} a_{k} \mathrm{H}^{2}\left(\mu_{1} * \delta_{x_{k}}, \mu_{2} * \delta_{x_{k}}\right) \\
& =\sum_{k} a_{k} \mathrm{H}^{2}\left(\mu_{1}, \mu_{2}\right)=v\left(\mathbb{R}^{d}\right) \mathrm{H}^{2}\left(\mu_{1}, \mu_{2}\right) .
\end{aligned}
$$

The general case then follows by approximating $v$ by a sequence of discrete measure $v_{n}$ converging to $v$ in $\mathcal{M}\left(\mathbb{R}^{d}\right)$ and observing that $\mu_{i} * v_{n} \rightarrow \mu_{i} * v$ weakly in $\mathcal{M}\left(\mathbb{R}^{d}\right)$. Since $\mathrm{HK}$ is weakly continuous by Theorem 7.15 , we obtain (8.95).

An easy application of the previous result is the contraction property of the (adjoint) Heat semigroup $\left(P_{t}^{*}\right)_{t \geq 0}$ in $\mathbb{R}^{d}$ with respect to $\mathrm{HK}$. In fact, we can prove a much more general result for the Heat flow in $\operatorname{RCD}(0, \infty)$ metric measure spaces $(X, \mathrm{~d}, m)[4,5]$. It covers the case of the semigroups $\left(P_{t}\right)_{t \geq 0}$ generated by

(A) the Heat equation on a open convex domain $\Omega \subset \mathbb{R}^{d}$ with homogeneous Neumann conditions

$$
\partial_{t} u=\Delta u \quad \text { in } \Omega \times(0, \infty), \quad \partial_{n} u=0 \quad \text { on } \partial \Omega \times(0, \infty),
$$

(B) the Heat equation on a complete Riemannian manifold $\left(\mathbb{M}^{d}, g\right)$ with nonnegative Ricci curvature defined by

$$
\partial_{t} u=\Delta_{g} u \text { in } \mathbb{M}^{d} \times(0, \infty),
$$

where $\Delta_{g}$ is the usual Laplace-Beltrami operator, and

(C) the Fokker-Planck equation in $\mathbb{R}^{d}$ generated by the gradient of a convex potentials $V: \mathbb{R}^{d} \rightarrow \mathbb{R}$, viz.

$$
\partial_{t} u=\Delta u-\nabla \cdot(u \mathrm{D} V) \text { in } \mathbb{R}^{d} \times(0, \infty) .
$$


Theorem 8.24 Let $(X, \mathrm{~d}, m)$ be a complete and separable metric-measure space with nonnegative Riemannian Ricci Curvature, i.e. satisfying the $\operatorname{RCD}(0, \infty)$ condition, and let $\left(P_{t}^{*}\right)_{t \geq 0}: \mathcal{M}(X) \rightarrow \mathcal{M}(X)$ be the Heat semigroup in the measure setting. Then

$$
\mathrm{HK}\left(P_{t}^{*} \mu_{1}, P_{t}^{*} \mu_{2}\right) \leq \mathrm{H}\left(\mu_{1}, \mu_{2}\right) \text { for all } \mu_{1}, \mu_{2} \in \mathcal{M}(X) \text { and } t>0 \text {. }
$$

Proof Recall that in $\operatorname{RCD}(0, \infty)$ metric measure spaces the $L^{2}$-gradient flow of the Cheeger energy induces a symmetric Markov semigroup $\left(P_{t}\right)_{t \geq 0}$ in $L^{2}(X, m)$ [4, Sect.6.1], which has a pointwise version satisfying the Feller regularization property $P_{t}\left(\mathrm{~B}_{b}(X)\right) \subset \operatorname{Lip}_{b}(X)$ for $t>0$ and the estimate (cf. [4, Thm. 6.2] or [5, Cor. 4.18])

$$
\left|\mathrm{D}_{X} P_{t} f\right|^{2}(x) \leq P_{t}\left(\left|\mathrm{D}_{X} f\right|^{2}\right)(x) \text { for } f \in \operatorname{Lip}_{b}(X), x \in X, t \geq 0 .
$$

Its adjoint $\left(P_{t}^{*}\right)_{t \geq 0}$ coincides with the Kantorovich-Wasserstein gradient flow in $\mathcal{P}_{2}(X)$ of the Entropy Functional $\mathscr{F}(\cdot \mid m)$ where $\mathscr{F}$ is induced by $F(s)=$ $U_{1}(s)=s \log s-s+1$ and defines a semigroup in $\mathcal{M}(X)$ by the formula

$$
\int_{X} f \mathrm{~d}\left(P_{t}^{*} \mu\right)=\int_{X} P_{t} f \mathrm{~d} \mu \text { for every } f \in \mathrm{B}_{b}(X) \text { and } \mu \in \mathcal{M}(X) .
$$

In order to prove (8.96) we use (8.54) (RCD-spaces satisfy the length property, [4, Thm. 5.1]) and apply $P_{t}$ to a subsolution $\left(\psi_{\theta}\right)_{\theta \in[0,1]}$ in $C^{1}\left([0,1] ; \operatorname{Lip}_{b}(X)\right)$ of the Hamilton-Jacobi equation

$$
\partial_{\theta} \psi_{\theta}+\frac{1}{4}\left|\mathrm{D}_{X} \psi_{\theta}\right|^{2}+\psi_{\theta}^{2} \leq 0 \text { in } X \times(0,1) .
$$

Since $P_{t}$ is a linear and continuous map from $\operatorname{Lip}_{b}(X)$ to $\operatorname{Lip}_{b}(X)$ the curve $\theta \mapsto \psi_{\theta, t}:=P_{t}\left(\psi_{\theta}\right)$ belongs to $C^{1}\left([0,1] ; \operatorname{Lip}_{b}(X)\right)$. Now, (8.97) and the Markov property yield

$$
\begin{aligned}
\left|\mathrm{D}_{X} P_{t} \psi_{\theta}\right|^{2}(x) & \leq P_{t}\left(\left|\mathrm{D}_{X} \psi_{\theta}\right|^{2}\right)(x), \\
\left(P_{t} \psi_{\theta}\right)^{2}(x) & \leq P_{t}\left(\psi_{\theta}^{2}\right)(x) \text { for } x \in X, \theta \in[0,1], t \geq 0 .
\end{aligned}
$$

Thus, for every $t \geq 0$ we obtain

$$
\partial_{\theta} \psi_{\theta, t}+\frac{1}{4}\left|\mathrm{D}_{X} \psi_{\theta, t}\right|^{2}+\psi_{\theta, t}^{2} \leq 0 \quad \text { in } X \times(0,1),
$$


and therefore

$$
\begin{aligned}
\int_{X} \psi_{1} \mathrm{~d}\left(P_{t}^{*}\right) \mu_{1}-\int_{X} \psi_{0} \mathrm{~d}\left(P_{t}^{*}\right) \mu_{0} \\
\quad=\int_{X} P_{t} \psi_{1} \mathrm{~d} \mu_{1}-\int_{X} P_{t} \psi_{0} \mathrm{~d} \mu_{0} \leq \mathrm{H}^{2}\left(\mu_{1}, \mu_{0}\right) .
\end{aligned}
$$

We conclude by taking the supremum with respect to all the subsolutions of (8.99) in $C^{1}\left([0,1] ; \operatorname{Lip}_{b}(X)\right)$ and applying (8.54).

Acknowledgements The quality of this manuscript considerably improved after the revision suggested by the anonymous reviewer, whose careful reading and constructive comments have been extremely helpful for the authors. The publication of this article was funded by the Open Access fund of the Weierstrass Institute.

Open Access This article is distributed under the terms of the Creative Commons Attribution 4.0 International License (http://creativecommons.org/licenses/by/4.0/), which permits unrestricted use, distribution, and reproduction in any medium, provided you give appropriate credit to the original author(s) and the source, provide a link to the Creative Commons license, and indicate if changes were made.

\section{A On the chronological development of our theory}

In this section we give a brief account of the order in which we developed the different parts of the theory. The beginning was the mostly formal work in [30] on reaction-diffusion systems, where a distance on vectors $\boldsymbol{u}$ of densities over a domain $\Omega \subset \mathbb{R}^{d}$ was formally defined in the Benamou-Brenier sense via

$$
\mathrm{d}\left(\boldsymbol{u}_{0}, \boldsymbol{u}_{1}\right)^{2}=\inf \int_{0}^{1} \int_{\Omega} \boldsymbol{\Xi}_{t}: \mathbb{M}_{\mathrm{diff}}\left(\boldsymbol{u}_{t}\right) \boldsymbol{\Xi}_{t}+\boldsymbol{\xi}_{t} \cdot \mathbb{K}_{\text {react }}\left(\boldsymbol{u}_{t}\right) \boldsymbol{\xi}_{t} \mathrm{~d} x \mathrm{~d} t
$$

under the constraint of the continuity equation $\partial_{t} \boldsymbol{u}_{t}+\nabla \cdot\left(\mathbb{M}_{\text {diff }}\left(\boldsymbol{u}_{t}\right) \boldsymbol{\Xi}_{t}\right)=$ $\mathbb{K}_{\text {react }}\left(\boldsymbol{u}_{t}\right) \boldsymbol{\xi}_{t}$. The central question was and still is the understanding of diffusion equations with reactions in the gradient-flow form $\partial_{t} \boldsymbol{u}=\nabla$. $\left(\mathbb{M}_{\text {diff }}(\boldsymbol{u}) \nabla \delta \mathcal{F}(\boldsymbol{u})\right)-\mathbb{K}_{\text {react }}(\boldsymbol{u}) \delta \mathcal{F}(\boldsymbol{u})$, see [30, Sect. 5.1]

It was natural to treat the scalar case first and to restrict to the case where both mobility operator $\mathbb{M}_{\text {diff }}(u)$ and $\mathbb{K}_{\text {react }}(u)$ are linear in $u$. Only in that case the formally derived system (1.29) for the geodesics $\left(u_{t}, \xi_{t}\right)$ decouples in the sense that $\xi_{t}$ solves an Hamilton-Jacobi equation that does not depend on $u$. Choosing $\mathbb{M}_{\text {diff }}(u)=\alpha u$ and $\mathbb{K}_{\text {react }}(u)=\beta u$ with $\alpha, \beta \geq 0$, the relevant Hamilton-Jacobi equation reads

$$
\partial_{t} \xi_{t}+\frac{\alpha}{2}\left|D_{x} \xi_{t}\right|^{2}+\frac{\beta}{2} \xi_{t}^{2}=0
$$


As in the other parts of this paper, we restrict to the case $\alpha=1$ and $\beta=4$ subsequently, but refer to [30] for the general case. Thus, the conjectured characterization (8.54) was first presented in Pisa at the Workshop "Optimal Transportation and Applications" in November 2012.

During a visit of the second author in Pavia, the generalized Hopf-Lax formula via the nonlinear convolution $\mathscr{P}_{t}$ (cf. (8.48)) was derived via the classical method of characteristics. This led to the unsymmetric representation (1.26) for H. To symmetrize this relation we used that $\mathscr{P}_{1} \xi(x)=\inf \Phi(\xi(y),|y-x|)$ with $\Phi(z, R)=\frac{1}{2}\left(1-\frac{A(R)}{1+2 z}\right)$, where $A(R)=\cos ^{2}(R \wedge(\pi / 2))$. Setting $\psi_{0}=-2 \xi_{0}$ and $\psi_{1}=2 \xi_{1}=2 \mathscr{P}_{1}$, we have the equivalence

$$
\xi_{1}=\mathscr{P}_{1} \xi_{0} \quad \Longleftrightarrow \quad\left(1-\psi_{0}\left(x_{0}\right)\right)\left(1-\psi_{1}\left(x_{1}\right)\right) \geq A\left(\left|x_{0}-x_{1}\right|\right) \text { for all } x_{i} .
$$

Setting $\varphi_{i}=-\log \left(1-\psi_{i}\right)$ we arrived at the cost function

$\mathrm{c}\left(x_{0}, x_{1}\right)=-\log A\left(\left|x_{0}-x_{1}\right|\right)= \begin{cases}-2 \log \left(\cos \left|x_{0}-x_{1}\right|\right) & \text { for }\left|x_{0}-x_{1}\right|<\pi / 2 \\ \infty & \text { otherwise }\end{cases}$

for the first time and obtained the characterization (1.7), namely

$$
\mathrm{H}\left(\mu_{0}, \mu_{1}\right)^{2}=\mathrm{D}\left(\mu_{0}, \mu_{1}\right)=\sup \left\{\mathscr{D}\left(\varphi_{0}, \varphi_{1} \mid \mu_{0}, \mu_{1}\right): \varphi_{0} \oplus \varphi_{1} \leq \mathrm{c}\right\} .
$$

It was then easy to dualize $\mathscr{D}$, and the Logarithmic Entropy functional $L E T$ in (1.20) was derived in July 2013.

While the existence of minimizers for $\operatorname{LET}\left(\mu_{0}, \mu_{1}\right)=\min \mathscr{E}\left(\gamma \mid \mu_{0}, \mu_{1}\right)$ was easily obtained, it was not clear at all, why and how $\mathrm{HK}$ defined via $\mathrm{HK}^{2}\left(\mu_{0}, \mu_{1}\right)=\min \mathscr{E}\left(\cdot \mid \mu_{0}, \mu_{1}\right)$ generates a geodesic distance. The only thing which could easily be checked was that the minimum was consistent with the distance between two Dirac masses, which could easily be calculated via the dynamic formulation.

So, in parallel we tried to develop the dynamic approach, which was not too successful at the early stages. Only after realizing and exploiting the connection to the cone distance in Summer and Autumn of 2013 we were able to connect LET systematically with the dynamic approach. The crucial and surprising observation was that optimal plans for $\mathscr{E}$ and lifts of measures $\mu \in \mathcal{M}(X)$ to measures $\lambda$ on the cone $\mathfrak{C}$ could be identified by exploiting the optimality conditions systematically. Corresponding results were presented in workshops on Optimal Transport in Banff (June 2014) and Pisa (November 2014).

Already at the Banff workshop, the general structure of the primal and dual Entropy-Transport problem as well as the homogeneous perspective formulation were presented. Several examples and refinements where developed afterwards. The most recent part from Summer 2015 concerns our Hamilton- 
Jacobi equation in general metric spaces $(X, \mathrm{~d})$ and the induced cone $\mathfrak{C}$ (cf. Sect. 8.4) and the derivation of the geodesic equations in $\mathbb{R}^{d}$ (cf. Sect. 8.6). This last achievement now closes the circle, by showing that all the initial steps, which were done on a formal level in 2012 and the first half of 2013, have indeed a rigorous interpretation.

\section{References}

1. Ambrosio, L., Erbar, M., Savaré, G.: Optimal transport, Cheeger energies and contractivity of dynamic transport distances in extended spaces. Nonlinear Anal. 137, 77-134 (2016)

2. Ambrosio, L., Gigli, N., Savaré, G.: Gradient Flows in Metric Spaces and in the Space of Probability Measures, 2nd ed. Lectures in Mathematics ETH Zürich, Birkhäuser Verlag, Basel (2008)

3. Ambrosio, L., Gigli, N., Savaré, G.: Calculus and heat flow in metric measure spaces and applications to spaces with Ricci bounds from below. Invent. Math. 195, 289-391 (2014)

4. Ambrosio, L., Gigli, N., Savaré, G.: Metric measure spaces with Riemannian Ricci curvature bounded from below. Duke Math. J. 163, 1405-1490 (2014)

5. Ambrosio, L., Gigli, N., Savaré, G.: Bakry-Émery curvature-dimension condition and Riemannian Ricci curvature bounds. Ann. Probab. 43, 339-404 (2015)

6. Ambrosio, L., Mondino, A., Savaré, G.: Nonlinear diffusion equations and curvature conditions in metric measure spaces, to appear on Memoirs of the A.M.S. (2015). arXiv: 1509.07273

7. Benamou, J.-D., Brenier, Y.: A computational fluid mechanics solution to the MongeKantorovich mass transfer problem. Numer. Math. 84, 375-393 (2000)

8. Brenier, Y., Otto, F., Seis, C.: Upper bounds on coarsening rates in demixing binary viscous liquids. SIAM J. Math. Anal. 43, 114-134 (2011)

9. Brézis, H.: Opérateurs maximaux monotones et semi-groupes de contractions dans les espaces de Hilbert. North-Holland Publishing Co, Amsterdam (1973). North-Holland Mathematics Studies, No. 5. Notas de Matemática (50)

10. Burago, D., Burago, Y., Ivanov, S.: A Course in Metric Geometry, vol 33 of Graduate Studies in Mathematics. American Mathematical Society, Providence (2001)

11. Burago, Y., Gromov, M., Perel'man, G.: A. D. Aleksandrov spaces with curvatures bounded below. Uspekhi Mat. Nauk 47, 3-51, 222 (1992)

12. Cheeger, J.: Differentiability of Lipschitz functions on metric measure spaces. Geom. Funct. Anal. 9, 428-517 (1999)

13. Chizat, L., Peyré, G., Schmitzer, B., Vialard, F.-X.: An interpolating distance between optimal transport and Fisher-Rao metrics. Found Comput Math (2016). https://doi.org/10. 1007/s10208-016-9331-y

14. Chizat, L., Peyré, G., Schmitzer, B., Vialard, F.-X.: Unbalanced optimal transport: geometry and Kantorovich formulation (2015). arXiv:1508.05216v1

15. Clarke, F .H.: Optimization and Nonsmooth Analysis. Wiley, New York (1983). A WileyInterscience Publication

16. Cordero-Erausquin, D., McCann, R.J., Schmuckenschläger, M.: A Riemannian interpolation inequality à la Borell, Brascamp and Lieb. Invent. Math. 146, 219-257 (2001)

17. Csiszár, I.: Information-type measures of difference of probability distributions and indirect observations. Studia Sci. Math. Hungar. 2, 299-318 (1967)

18. Dolbeault, J., Nazaret, B., Savaré, G.: A new class of transport distances between measures. Calc. Var. Part. Differ. Equ. 34, 193-231 (2009) 
19. Dudley, R .M.: Real Analysis and Probability, vol. 74 of Cambridge Studies in Advanced Mathematics. Cambridge University Press, Cambridge (2002). Revised reprint of the 1989 original

20. Ekeland, I., Temam, R.: Analyse Convexe et Problèmes Variationnels. Dunod, GauthierVillars (1974)

21. Erbar, M., Kuwada, K., Sturm, K.-T.: On the equivalence of the entropic curvaturedimension condition and Bochner's inequality on metric measure spaces. Invent. Math. 201, 993-1071 (2015)

22. Hellinger, E.: Neue Begründung der Theorie quadratischer Formen von unendlichvielen Veränderlichen. J. Reine Angew. Math. 136, 210-271 (1909)

23. Hiriart-Urruty, J.-B., Lemaréchal, C.: Convex analysis and minimization algorithms. I, vol. 305 of Grundlehren der Mathematischen Wissenschaften [Fundamental Principles of Mathematical Sciences]. Springer, Berlin (1993)

24. Jordan, R., Kinderlehrer, D., Otto, F.: The variational formulation of the Fokker-Planck equation. SIAM J. Math. Anal 29, 1-17 (1998). (electronic)

25. Kakutani, S.: On equivalence of infinite product measures. Ann. Math. (2) 49, 214-224 (1948)

26. Kellerer, H.G.: Duality theorems for marginal problems. Z. Wahrsch. Verw. Gebiete 67, 399-432 (1984)

27. Kondratyev, S., Monsaingeon, L., Vorotnikov, D.: A new optimal transport distance on the space of finite Radon measures. Adv. Differ. Equ. 21, 1117-1164 (2016)

28. Kullback, S., Leibler, R.A.: On information and sufficiency. Ann. Math. Stat. 22, 79-86 (1951)

29. LeCam, L.: Convergence in distribution of stochastic processes. Univ. Calif. Publ. Stat. 2 , 207-236 (1957)

30. Liero, M., Mielke, A., Savaré, G.: Optimal transport in competition with reaction-the Hellinger-Kantorovich distance and geodesic curves. SIAM J. Math. Anal. 48, 2869-2911 (2016)

31. Liese, F., Vajda, I.: On divergences and informations in statistics and information theory. IEEE Trans. Inf. Theory 52, 4394-4412 (2006)

32. Lin, J.: Divergence measures based on the Shannon entropy. IEEE Trans. Inf. Theory 37, 145-151 (1991)

33. Lisini, S.: Characterization of absolutely continuous curves in Wasserstein spaces. Calc. Var. Part. Differ. Equ. 28, 85-120 (2007)

34. Lott, J., Villani, C.: Ricci curvature for metric-measure spaces via optimal transport. Ann. Math. (2) 169, 903-991 (2009)

35. McCann, R.J.: A convexity principle for interacting gases. Adv. Math. 128, 153-179 (1997)

36. Otto, F.: The geometry of dissipative evolution equations: the porous medium equation. Commun. Part. Differ. Equ. 26, 101-174 (2001)

37. Otto, F., Villani, C.: Generalization of an inequality by Talagrand and links with the logarithmic Sobolev inequality. J. Funct. Anal. 173, 361-400 (2000)

38. Piccoli, B., Rossi, F.: Generalized Wasserstein distance and its application to transport equations with source. Arch. Ration. Mech. Anal. 211, 335-358 (2014)

39. Piccoli, B., Rossi, F.: On properties of the Generalized Wasserstein distance. Arch. Ration. Mech. Anal. 222, 1339-1365 (2016)

40. Rachev, S .T., Rüschendorf, L.: Mass Transportation Problems. Vol. I-II, Probability and Its Applications. Springer, New York (1998)

41. Rockafellar, R.T.: Convex Analysis. Princeton University Press, Princeton (1970)

42. Santambrogio, F.: Optimal Transport for Applied Mathematicians. Springer, New York (2015)

43. Schachermayer, W., Teichmann, J.: Characterization of optimal transport plans for the Monge-Kantorovich problem. Proc. Am. Math. Soc. 137, 519-529 (2009) 
44. Schwartz, L.: Radon Measures on Arbitrary Topological Spaces and Cylindrical Measures. Published for the Tata Institute of Fundamental Research, Bombay by Oxford University Press, London (1973). Tata Institute of Fundamental Research Studies in Mathematics, No. 6

45. Simons, S.: Minimax and Monotonicity, vol. 1693 of Lecture Notes in Mathematics. Springer, Berlin (1998)

46. Sturm, K.-T.: Metric spaces of lower bounded curvature. Expos. Math. 17, 35-47 (1999)

47. Sturm, K.-T.: On the geometry of metric measure spaces. I. Acta Math. 196, 65-131 (2006)

48. Sturm, K.-T.: On the geometry of metric measure spaces. II. Acta Math. 196, 133-177 (2006)

49. Villani, C.: Topics in Optimal Transportation, vol. 58 of Graduate Studies in Mathematics. American Mathematical Society, Providence (2003)

50. Villani, C.: Optimal Transport. Old and New, vol. 338 of Grundlehren der Mathematischen Wissenschaften. Springer, Berlin (2009) 